\title{
ENERGY CONSERVATION MECHANISMS AND ELECTRON \\ TRANSFER IN SYNTROPHIC PROPIONATE-OXIDIZING MICROBIAL CONSORTIA
}

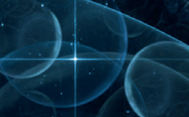




\section{PROPOSITIONS}

1. Reverse electron transfer, exocellular electron transfer and confurcation mechanisms are all necessary for syntrophic growth on propionate (This thesis)

2. In Syntrophobacter fumaroxidans, molybdenum-containing formate dehydrogenases are key enzymes for interspecies electron transfer during syntrophic propionate oxidation (This thesis)

3. Anaerobic digestion is no longer a black-box but rather an overexposed photo.

4. The circadian rhythm in archaea and bacteria will become essential in the planning of experiments. (Johnson et al., 2017. Nature Reviews Microbiology)

5. The discovery of the existence of water as two fluids with different densities at the same time, as described by Perakis et al. (PNAS. 2017), has major implications in metabolism.

6. Scientists are to blame for the wave of antiscientific policies around the world.

7. The title of Philosophiae Doctor (PhD) does not describe the current aptitudes of $\mathrm{PhD}$ graduates.

8. In the quest for practicality and universal understanding, the scientific language has become tedious.

Prepositions belonging to the PhD thesis entitled "Energy conservation mechanisms and electron transfer in syntrophic propionate-oxidizing microbial consortia".

Vicente T. Sedano Núñez

Wageningen, 3 April 2018 


\title{
ENERGY CONSERVATION MECHANISMS AND ELECTRON TRANSFER IN SYNTROPHIC PROPIONATE-OXIDIZING MICROBIAL CONSORTIA
}

\author{
VICENTE T. SEDANO NÚÑEZ
}




\section{Thesis committee}

\section{Promotor}

Prof. Dr Alfons J.M. Stams

Personal chair at the Laboratory of Microbiology

Wageningen University \& Research

\section{Co-promotor}

Dr Caroline M. Plugge

Associate professor, Laboratory of Microbiology

Wageningen University \& Research

\section{Other members}

Prof. Dr Willem J.H. van Berkel, Wageningen University \& Research

Prof. Dr Huub J.M. Op den Camp, Radboud University Nijmegen, The Netherlands

Dr Robbert Kleerebezem, Delft University of Technology, The Netherlands

Prof. Dr Inês Cardoso Pereira, Universidade Nova de Lisboa, Portugal

This research was conducted under the auspices of the Graduate School for Socio-Economic and Natural Sciences of the Environment (SENSE). 


\section{Energy conservation mechanisms and electron transfer in syntrophic propionate-oxidizing microbial consortia}

\section{Vicente T. Sedano Núñez}

\section{Thesis}

submitted in fulfilment of the requirements for the degree of doctor

at Wageningen University

by the authority of the Rector Magnificus,

$$
\begin{aligned}
& \text { Prof. Dr A.P.J. Mol, } \\
& \text { in presence of the }
\end{aligned}
$$

Thesis Committee appointed by the Academic Board

to be defended in public

on Tuesday 3 April 2018

at $1: 30$ p.m. in the Aula. 
Vicente Tonamellotl Sedano Núñez

Energy conservation mechanisms and electron transfer in syntrophic propionate-oxidizing microbial consortia,

206 pages.

PhD thesis, Wageningen University, Wageningen, the Netherlands (2018)

With references, with summary in English

ISBN: 978-94-6343-263-4

DOI: $10.18174 / 441473$ 
To the two people that inspired me to begin a doctorate:

S.S.R. \& A.H.G.

And to the two people that inspired me to complete it:

G.S.C. \& N.A.O. 



\section{Table of contents}

$\begin{array}{lll}\text { Chapter } 1 \text { General introduction and thesis outline } & 10\end{array}$

Chapter 2 A genomic comparison of syntrophic and non-syntrophic 28 butyrate- and propionate-degrading bacteria points to a key role of formate in syntrophy

Chapter 3 Metabolic flexibility of Syntrophobacter fumaroxidans: 54 Syntrophic vs sulfate-reducing lifestyle during growth on propionate

Chapter 4 Comparative proteome analysis of propionate 74 degradation by Syntrophobacter fumaroxidans in pure culture and in coculture with methanogens

Chapter 5 Proteomic analyses of Methanospirillum hungatei and 110 Methanobacterium formicicum grown in a syntrophic partnership with Syntrophobacter fumaroxidans and in pure culture with $\mathrm{H}_{2} / \mathrm{CO}_{2}$ or formate.

Chapter 6 Microbial community analysis and performance of a 140 mesophilic anaerobic membrane bioreactor treating pot ale

Chapter 7 General discussion

References

\section{Appendices}

Summary

Acknowledgments

202

SENSE diploma 

"There are two mistakes one can make along the road... Not going all the way, and not starting" S. G. 

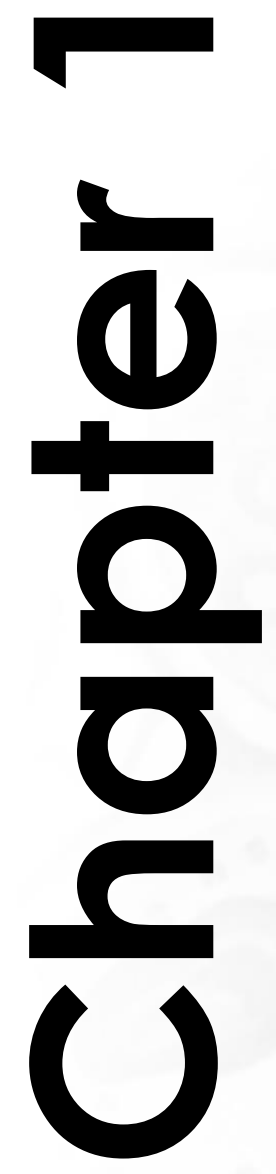
General introduction

$$
\text { and thesis outline }
$$


Anaerobic degradation of organic matter to carbon dioxide and methane requires the interaction of different microbial groups along a series of four stages: hydrolysis, acidogenesis, acetogenesis and methanogenesis (McInerney et al., 2008; Sieber et al., 2012; Shrestha and Rotaru, 2014) (Figure 1.1). During hydrolysis and acidogenesis, complex organic structures like polysaccharides, proteins and lipids are converted to smaller moieties such as organic acids, alcohols, simple sugars and amino acids, among others. Several of these products can be directly mineralized to $\mathrm{CO}_{2}$ by microorganisms using inorganic electron acceptors such as iron $\left(\mathrm{Fe}^{3+}\right)$, manganese $\left(\mathrm{Mn}^{2+}\right)$, nitrate $\left(\mathrm{NO}_{3}{ }^{-}\right)$, sulfate $\left(\mathrm{SO}_{4}{ }^{2-}\right)$, selenate $\left(\mathrm{SeO}_{4}{ }^{2-}\right)$ or arsenate $\left(\mathrm{AsO}_{4}{ }^{3-}\right)$ (Kristensen et al., 1995; Stams et al., 2006).

\section{Polymers}

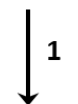

Hydrolysis

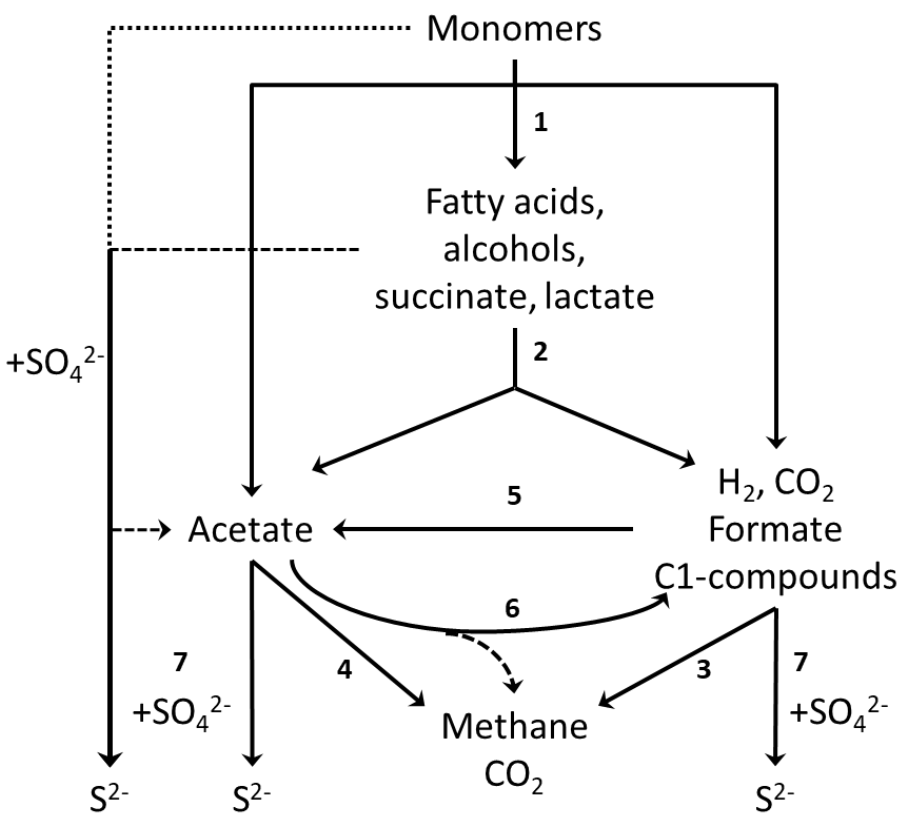

Acidogenesis

Acetogenesis

Methanogenesis

Sulfidogenesis

Figure 1.1. Anaerobic degradation of organic matter by cooperation of different metabolic groups. Primary fermenters (1), secondary fermenters or acetogens (2), hydrogenotrophic methanogens (3), acetoclastic methanogens, homoacetogenic bacteria (5), syntrophic acetate oxidizers (6) and sulfate reducers (7).

However, in some environments, microcosms or even in man-made environments, such as paddy fields, landfills and anaerobic bioreactors, inorganic electron acceptors are not always sufficiently available, or the products of their reduction are not desirable, for instance, sulfide production from sulfate reduction. In such 
environments, where inorganic electron acceptors are limited, the degradation of organic matter is channelled to methane production. Consequently, syntrophic associations between secondary fermentative bacteria and methanogens become important. These associations occur since the degradation of fatty acids by acetogenic bacteria, requires that the products, typically hydrogen, formate and acetate, are maintained at very low concentrations by methanogenic archaea (Stams et al., 2005; McInerney et al., 2009; Schink and Stams, 2013).

\section{Energetic constraints in methanogenic ecosystems}

During hydrolysis, polymeric compounds such as carbohydrates, proteins and lipids are converted to sugars, amino acids and fatty acids. These monomeric compounds are in turn fermented in the acidogenic stage to volatile fatty acids, alcohols, succinate, lactate, $\mathrm{H}_{2}, \mathrm{CO}_{2}$, and $\mathrm{H}_{2} \mathrm{~S}$. The subsequent degradation of these products is an important limiting step in anaerobic digestion due to thermodynamic constrains. Most of the secondary fermentative conversions proceed close to thermodynamic equilibrium with Gibbs energy changes close to zero (Table 1.1) (Thauer et al., 1977; Schink, 1997; Kleerebezem and Stams, 2000).

It can be noticed from Table 1.1 that the energetic barriers for anaerobic lactate and ethanol oxidation are easier to overcome than those for butyrate, propionate and acetate degradation. Degradation of butyrate, propionate and acetate in the absence of inorganic electron acceptors can only occur when the concentrations of hydrogen and formate are low, $<10 \mathrm{~Pa}$ and $<10 \mu \mathrm{M}$, respectively (Stams, 1994). When oxidation of these organic acids is coupled to methane production in a tight association with a methanogenic partner, the acetogenic reactions mentioned above become thermodynamically favourable (Equations 1 and 2). Having this in mind, a complete oxidation of butyrate and propionate into methane and $\mathrm{CO}_{2}$ requires the interaction of at least three different groups of microorganisms: acetogenic bacteria, acetotrophic archaea and hydrogenotrophic archaea. This interaction is more than simple mutualism as it is based on providing trophic benefits for all the involved partners.

$$
\begin{aligned}
& 2 \text { Butyrate }^{-}+5 \mathrm{H}_{2} \mathrm{O} \rightarrow 5 \mathrm{CH}_{4}+3 \mathrm{HCO}_{3}{ }^{-}+\mathrm{H}^{+} \quad \Delta \mathrm{G}^{\circ}=-177 \mathrm{~kJ} / \mathrm{reac} \quad \text { Eq. } 1 \\
& 4 \text { Propionate }^{-}+7 \mathrm{H}_{2} \mathrm{O} \rightarrow 7 \mathrm{CH}_{4}+5 \mathrm{HCO}_{3}{ }^{-}+\mathrm{H}^{+} \quad \Delta \mathrm{G}^{\circ}=-249 \mathrm{~kJ} / \mathrm{reac} \quad \text { Eq. } 2
\end{aligned}
$$

Not only acetogenic bacteria depend on efficient removal of the products formed by oxidation of organic acids, but methanogenic archaea rely on the acetogenic partner for substrate supply since methanogens are able to convert only a small number of one-carbon compounds (e.g. methanol, formate, methylamines and methylsulfides) besides acetate (Zinder, 1993; Liu and Whitman, 2008; Thauer et al., 2008). 
Table 1.1. Equations and standard free energy changes for acetogenic and methanogenic reactions. Values were calculated from the Gibbs free energies of formation of the reactants at a concentration of $1 \mathrm{M}, \mathrm{pH} 7.0,298 \mathrm{~K}$ and a partial pressure of gas of $10^{5} \mathrm{~Pa}$ according to Thauer et al. 1977 .

\begin{tabular}{|c|c|c|}
\hline \multicolumn{2}{|c|}{ Acetogenic reactions } & $\Delta \mathrm{G}^{\mathrm{ol}_{1}}(\mathrm{~kJ} / \mathrm{mol})$ \\
\hline Lactate $^{-}+2 \mathrm{H}_{2} \mathrm{O}$ & $\rightarrow$ Acetate $^{-}+\mathrm{HCO}_{3}+\mathrm{H}^{+}+2 \mathrm{H}_{2}$ & -7.7 \\
\hline Ethanol $+\mathrm{H}_{2} \mathrm{O}$ & $\rightarrow$ Acetate $^{-}+\mathrm{H}^{+}+2 \mathrm{H}_{2}$ & +9.6 \\
\hline Butyrate $^{-}+2 \mathrm{H}_{2} \mathrm{O}$ & $\rightarrow 2$ Acetate $^{-}+\mathrm{H}^{+}+2 \mathrm{H}_{2}$ & +48.6 \\
\hline Butyrate $^{-}+2 \mathrm{HCO}_{3}^{-}$ & $\rightarrow 2$ Acetate $^{-}+\mathrm{H}^{+}+2$ Formate $^{-}$ & +45.6 \\
\hline Propionate $^{-}+3 \mathrm{H}_{2} \mathrm{O}$ & $\rightarrow$ Acetate $^{-}+\mathrm{HCO}_{3}^{-}+\mathrm{H}^{+}+3 \mathrm{H}_{2}$ & +76.1 \\
\hline Propionate $^{-}+2 \mathrm{HCO}_{3}^{-}$ & $\rightarrow$ Acetate $^{-}+\mathrm{H}^{+}+3$ Formate $^{-}$ & +72.2 \\
\hline \multicolumn{2}{|c|}{ Hydrogen/formate formation reactions } & $\Delta \mathrm{G}^{\mathrm{o}^{\prime}}(\mathrm{kJ} / \mathrm{mol})$ \\
\hline Acetate $^{-}+4 \mathrm{H}_{2} \mathrm{O}$ & $\rightarrow 4 \mathrm{H}_{2}+2 \mathrm{HCO}_{3}^{-}+\mathrm{H}^{+}$ & +95.0 \\
\hline Acetate $^{-}+2 \mathrm{HCO}_{3}^{-}$ & $\rightarrow 4$ Formate $^{-}+\mathrm{H}^{+}$ & +109 \\
\hline Formate $^{-}+\mathrm{H}_{2} \mathrm{O}$ & $\rightarrow \mathrm{H}_{2}+\mathrm{HCO}_{3}^{-}$ & +1.3 \\
\hline \multicolumn{2}{|c|}{ Methanogenic reactions } & $\Delta G^{01}(\mathrm{~kJ} /$ reac) \\
\hline 4 Formate $^{-}+\mathrm{H}^{+}+\mathrm{H}_{2} \mathrm{O}$ & $\rightarrow \mathrm{CH}_{4}+3 \mathrm{HCO}_{3}^{-}$ & -130.4 \\
\hline $4 \mathrm{H}_{2}+\mathrm{HCO}_{3}^{-}+\mathrm{H}^{+}$ & $\rightarrow \mathrm{CH}_{4}+3 \mathrm{H}_{2} \mathrm{O}$ & -135.6 \\
\hline Acetate $^{-}+\mathrm{H}_{2} \mathrm{O}$ & $\rightarrow \mathrm{CH}_{4}+\mathrm{HCO}_{3}^{-}$ & -31.0 \\
\hline
\end{tabular}

\section{Methanogenic syntrophy}

Methanogenic syntrophy is an essential process in the global carbon cycle and fundamental for the complete mineralization of organic carbon (Thauer et al., 2008; McInerney et al., 2009). The mutual dependence of syntrophic partners with respect to energy limitations can go, in some cases, so far that neither partner can operate without the other, and together they exhibit a metabolic activity that neither one could accomplish on its own (de Bok et al., 2005; Qiu et al., 2006; Sousa et al., 2007; McInerney et al., 2008).

A balanced performance among the trophic groups of microorganisms involved in syntrophy depends on many factors such as the concentration of substrates and products as well as their diffusion, intermicrobial distances, formation of aggregates, etc. (Seitz et al., 1988; Thiele et al., 1988; Boone et al., 1989; Ozturk et al., 1989; Stams and Dong, 1995).

Although the driving force for the establishment of syntrophic associations is the small amount of energy available in methanogenic environments, these associations seems to be very difficult to achieve and easy to disrupt. The mechanisms by which syntrophic microorganisms thrive with a very low energy gain, which furthermore must be shared among different cells, are very complex and have not been completely understood yet. 


\section{Interspecies electron transfer (IET)}

The transfer of reducing equivalents in syntrophic consortia is denoted as interspecies electron transfer (Stams, 1994; Schink, 1997; Stams and Plugge, 2009; Sieber et al., 2014). Several redox shuttles have been recognized to be involved in IET, among them, humic substances (Lovley et al., 1999), sulfur compounds (Milucka et al., 2012), cysteine-cystine shuttles (Kaden et al., 2002), flavins (Von Canstein et al., 2008; Brutinel and Gralnick, 2012) and conductive particles (Chen et al., 2014; Cruz Viggi et al., 2014; Kato, 2015; Rotaru et al., 2017). However, the interspecies exchange of hydrogen and formate has been the most widely studied. Hydrogen and formate are formed by secondary fermenting bacteria to release the excess of electrons during the oxidative degradation of organic acids (Schink, 1997; McInerney et al., 2009; Stams and Plugge, 2009; Schink et al., 2017).

Reducing equivalents are formed along different oxidation steps in the anaerobic degradation of organic compounds. The reduced forms of electron carriers, such as $\mathrm{NADH}$ or reduced ferredoxin $\left(\mathrm{Fd}_{\mathrm{red}}\right)$ need to be re-oxidized to keep the different pathways functioning. During acidogenesis the oxidation of NADH (E' of $-320 \mathrm{mV}$ ) coupled to the reduction of acetaldehyde (E' of $-197 \mathrm{mV})$, pyruvate (E' of $-190 \mathrm{mV})$, enoyl-CoA (E' of $-10 \mathrm{mV})$, or fumarate (E' of $+33 \mathrm{mV})$, is energetically favourable, allowing primary fermentative bacteria to form ethanol, lactate, butyrate, or propionate, respectively.

However, in acetogenesis the re-oxidation of the electron carriers, in the absence of nitrate, sulfate or other external electron acceptor, needs to be coupled to protons and $\mathrm{CO}_{2}$ reduction leading to the formation of hydrogen and formate. (McInerney et al., 2008). It is energetically difficult to reduce protons using the redox mediators $\mathrm{NADH}$ and ferredoxin. The midpoint redox potential $\left(\mathrm{E}^{\circ}\right)$ of the redox couples $\mathrm{NAD}^{+} / \mathrm{NADH}$ and $\mathrm{Fd}_{\mathrm{ox}} / \mathrm{Fd}_{\text {red }}$ is -320 and $-398 \mathrm{mV}$, respectively; while the $\mathrm{E}^{\text {or }}$ of the redox couples $\mathrm{H}^{+} / \mathrm{H}_{2}$ and $\mathrm{CO}_{2} / \mathrm{HCOO}$ is much lower with $-414 \mathrm{mV}$ and $-432 \mathrm{mV}$, respectively (Thauer et al., 1977). This causes an energetic problem under standard conditions (for comparison, the $\mathrm{E}^{\mathrm{ol}}$ of the redox couple $\mathrm{O}_{2} / \mathrm{H}_{2} \mathrm{O}$, which is important for aerobic respiration, is $+818 \mathrm{mV}$ ). In nature however, methanogens can maintain hydrogen threshold values below $10 \mathrm{~Pa}$ and formate concentrations as low as $10 \mu \mathrm{M}$ (Stams, 1994). At these levels, the redox potential for hydrogen and formate production changes from -414 and $-432 \mathrm{mV}$ to -260 and $-290 \mathrm{mV}$, respectively (Sieber et al., 2012). Thus, hydrogen and formate production from $\mathrm{NADH}$ and $\mathrm{Fd}$ red becomes thermodynamically favourable. Consequently, in anaerobic environments, longchain fatty acids, butyrate, propionate, alcohols, and some amino acids and aromatic compounds are syntrophically degraded to the methanogenic substrates, $\mathrm{H}_{2}$, formate, and acetate (McInerney et al., 2008). 


\section{Hydrogen and formate as electron carriers}

Since Bryant and co-workers discovered that their cultures of Methanobacillus omelianskii did not contain one species but a syntrophic coculture of two types of microorganisms, hydrogen and formate were recognized from the very beginning as key metabolites in the electron transfer processes between the ethanol oxidizing bacterium and the methanogenic partner (Bryant et al., 1967). Since then, many syntrophic associations have been described and the debate over which compound is the main interspecies electron carrier between the diverse syntrophic associations continues (McInerney et al., 2009; Sieber et al., 2012; Morris et al., 2013; Schink et al., 2017).

Many studies in syntrophic metabolism have emphasized the role of hydrogen in IET because the methanogens used in partnerships with Pelotomaculum thermopropionicum, Syntrophococcus sucromutans, Syntrophomonas wolfei, Syntrophobotulus glycolicus and Thermoacetogenium phaeum among others, were only able to utilize $\mathrm{H}_{2} / \mathrm{CO}_{2}$ and not formate (Schink, 1997; Sieber et al., 2012). However, other studies have shown the significance of formate transfer in methanogenic communities (Thiele and Zeikus, 1988; Boone et al., 1989; Schmidt and Ahring, 1995; de Bok et al., 2003) and in cocultures with Syntrophobacter fumaroxidans (Dong and Stams, 1995; Stams and Dong, 1995; de Bok et al., 2002a; de Bok et al., 2003), Desulfovibrio desulfuricans strain G20 (Li et al., 2009), Syntrophomonas wolfei and Syntrophus aciditrophicus (Sieber et al., 2014). Thus, although the exclusive use of hydrogen has been reported for the butyrate-oxidizing coculture Syntrophomonas wolfei (Wofford et al., 1986), biochemical and genomic information supports the combined occurrence of hydrogen and formate transfer (Walker et al., 2009; Hillesland and Stahl, 2010; Müller et al., 2010; Li et al., 2011; Worm et al., 2011b; Rotaru et al., 2012; Schink and Stams, 2013).

Therefore, the production and oxidation of hydrogen and formate plays a crucial role in the metabolism of syntrophic methanogenic microorganisms. Hydrogen formation is one of the simplest redox reactions in nature, which nevertheless requires enzymes with complex active centres (Hedderich and Forzi, 2005; Vignais and Billoud, 2007). Hydrogenases are metalloenzymes that catalyse the reversible conversion of protons and electrons into molecular hydrogen (Equation 3) (Lubitz et al., 2014).

$$
\mathrm{H}_{2} \rightleftharpoons 2 \mathrm{H}^{+}+2 \mathrm{e}^{-} \quad \text { Eq. } 3
$$

Hydrogenases are widespread in nature; they occur in bacteria, archaea, and eukarya. It is predicted that over 55 microbial phyla and over a third of all microorganisms harbour hydrogenases. There are three different types of hydrogenases known to date and their current classification is based on the active site metal composition (Søndergaard et al., 2016). 
1. [NiFe]-Hydrogenases. Heterodimeric proteins consisting of small and large subunits. The large subunit contains the active site, a sulfur bridged bimetallic centre of iron and nickel typically with an open coordination site on one metal. The small subunit contains one or more Fe-S clusters.

2. [FeFe]-Hydrogenases. In these enzymes, which can be monomeric or heterodimeric, catalysis occurs at a unique di-iron centre containing a bridging dithiolate ligand, three $\mathrm{CO}$ ligands and two $\mathrm{CN}$ - ligands (Berggren et al., 2013). The active site of the [FeFe]-hydrogenase is the $\mathrm{H}$ cluster, which consists of a $[4 \mathrm{Fe}-4 \mathrm{~S}]_{\mathrm{H}}$ subcluster linked to an organometallic $[2 \mathrm{Fe}]_{\mathrm{H}}$ subcluster (Suess et al., 2016).

3. [Fe]-Hydrogenases. Iron-only hydrogenases, also called iron-sulfurcluster-free hydrogenases, these enzymes have been found only in some hydrogenotrophic methanogenic archaea, containing neither nickel nor ironsulfur clusters but only an iron-containing cofactor.

[NiFe]-hydrogenases have been reported to be often more active towards $\mathrm{H}_{2}$ oxidation and the [ $\mathrm{FeFe}]$-hydrogenases extremely active in $\mathrm{H}_{2}$ generation (Wu and Mandrand, 1993; Vignais et al., 2001). Nevertheless, [FeFe]-hydrogenase have the highest $\mathrm{H}_{2}$ production activities (Cammack, 1999; Hambourger et al., 2008; Brown et al., 2012). Although the catalytic activities reported for [ $\mathrm{NiFe}]$-hydrogenases are usually lower (Lubitz et al., 2014), these enzymes show much less sensitivity to oxygen inactivation and can generally recover from it, unlike [ $\mathrm{FeFe}]$-hydrogenases which are extremely sensitive and irreversible inactivated by oxygen in the reduced state (Vincent et al., 2007; Lenz et al., 2010).

A subgroup of the [NiFe]-Hydrogenases is formed by the [NiFeSe]-Hydrogenases, in which one of the cysteine ligands of the nickel is replaced by a selenocysteine (Sec) (Garcin et al., 1999). Although the chemical properties of selenium and sulfur are similar, the change of coordination caused by replacement of one cysteine by a selenocysteine modifies considerably the catalytic and spectroscopic features of the active site (Baltazar et al., 2011). Recent studies have shown that selenium has a crucial role in protection against oxidative damage and that [NiFeSe]-hydrogenases have a higher catalytic activity than [NiFe]-hydrogenases and a bias towards $\mathrm{H}_{2}$ production (Ceccaldi et al., 2015; Marques et al., 2017; Ruff et al., 2017). Another classification (biochemical) of hydrogenases is often made according to its redox partner, which in many cases is $\mathrm{NAD}(\mathrm{P})^{+}$, ferredoxin, coenzyme $\mathrm{F}_{420}$ or a-, b- or c-type cytochrome (Vignais and Billoud, 2007). The metal sites of the three types of hydrogenases involved in interspecies hydrogen transfer have unusual structural features in common, such as intrinsic CO ligands. Despite this, [NiFe]-hydrogenases, $[\mathrm{FeFe}]$-hydrogenases, and [Fe]-hydrogenases are not phylogenetically related at the level of their primary structure or at the level of the enzymes involved in their activesite biosynthesis. 
Formate dehydrogenases (FDHs) comprise a heterogeneous group of enzymes found in both prokaryotes and eukaryotes that catalyse the reversible oxidation of formate to $\mathrm{CO}_{2}$ and $\mathrm{H}^{+}$.

$$
\mathrm{HCOO}^{-} \rightleftharpoons \mathrm{CO}_{2}+\mathrm{H}^{+}+2 \mathrm{e}^{-} \quad \text { Eq. } 4
$$

The most common class is present in aerobic organisms, monomeric, without cofactors in the active site and mainly $\mathrm{NAD}(\mathrm{P})^{+}$-dependent (Ferry, 1990). However, the conversion of $\mathrm{CO}_{2}$ to formate results difficult in aerobic conditions because the redox potential of $\mathrm{NADP}^{+}$is more positive than that of $\mathrm{CO}_{2}$ (Reda et al., 2008). In prokaryotes, on the other hand, formate dehydrogenases reversibly interconvert $\mathrm{CO}_{2}$ and formate; they can be found in heterodimeric form (in subunits $\alpha, 8$, and Y), and contain a complex inventory of redox centres sensitive to oxygen (Reda et al., 2008; Bassegoda et al., 2014). The active sites of formate dehydrogenases from anaerobic prokaryotes include transition metals, such as molybdenum (Mo), tungsten (W) and non-heme iron, molybdopterin guanine dinucleotides as cofactors and in some cases a Sec residue. Inactivation by cyanide can be partially reversed by incubation with sulfide (Robinson et al., 2017). The cofactors are used to transfer the electrons from formate oxidation to an independent active site, to reduce quinone, protons, or $\mathrm{NAD}(\mathrm{P})^{+}$(Reda et al., 2008). The high efficiency and specificity of isolated bacterial formate dehydrogenases have become appealing for environmental and industrial applications. There is increasing interest in developing biocatalysts that remove $\mathrm{CO}_{2}$ electrochemically from the atmosphere as a mean of relieving global warming while producing fuels or chemical feedstocks (El-Zahab et al., 2008; Yadav et al., 2012; Sakai et al., 2017)

Hydrogenases and formate dehydrogenases are located in the periplasm or cytoplasm, either in soluble form or membrane-bound. Their location is important when analysing their role in interspecies electron transfer. Depending on the location in the cell, these enzymes may either be tuned for hydrogen/formate production, removing reducing equivalents, or hydrogen/formate uptake, providing electrons to the cell.

The importance of hydrogen and formate as electron carriers is not limited to IET, but they are also used in other energy conservation mechanisms such as reverse electron transport and bifurcating/confurcating complexes that will be discussed below. Moreover, IET is not restricted either to methanogenic systems, as the research in other syntrophic cocultures indicate, for instance in Pelobacter carbinolicus and Geobacter sulfurreducens (Rotaru et al., 2012). 


\section{Direct interspecies electron transfer (DIET)}

A direct interspecies electron transfer mechanism in a coculture of Geobacter metallireducens and Geobacter sulfurreducens growing on ethanol and fumarate has been described (Summers et al., 2010). Direct interspecies electron transfer (DIET) has been proposed before in G. sulfurreducens and Shewanella oneidensis (Reguera et al., 2005; Gorby et al., 2006), where the authors proposed that electrically conductive pili-like appendages, termed nanowires, were involved in electron transferring from the cell surface to the surface of Fe(III) oxides. The mechanisms underlying DIET are still being investigated and debated (Reguera et al., 2005; Lovley and Malvankar, 2015). Besides the production of pili or nanowires as the structures utilized to transfer the electrons among cells, the requirement of a multitude of extracellular and periplasmic cytochromes has also been suggested (Larsen et al., 2015; Lovley, 2017). The first studies in DIET were limited to interactions of metal-reducing bacteria, such as Geobacter and Shewanella species, in which one bacteria oxidized ethanol while the other reduced fumarate (Liu et al., 2012; Shrestha et al., 2013; Rotaru et al., 2014). More recently DIET has been investigated in methanogenic environments for syntrophic associations between Geobacter species and species of Methanosaeta and Methanothrix (Rotaru et al., 2014; Holmes et al., 2017; Rotaru et al., 2017).

Although DIET might indeed play a role in syntrophic communities of anaerobic microorganisms, hydrogen and formate transfer among acetogenic bacteria and methanogens remains the best-established mechanism for interspecies electron transfer.

\section{Energy conservation mechanisms in anaerobic environments}

Conservation of energy by chemotrophic microorganisms is based on both substratelevel phosphorylation and electron transport phosphorylation (Stams et al., 2006). We have explained the energetic limitations faced by acetogenic bacteria in the absence of inorganic electron acceptors; and how IET and the establishment of syntrophic associations makes, the otherwise thermodynamically unfavourable, reduction of protons with NADH possible. But even under optimal syntrophic growth conditions, when hydrogen, formate, and acetate are low, the Gibbs free energy change for syntrophic metabolism is very close to the minimum increment of energy required for ATP synthesis, which is predicted to be about -15 to $-20 \mathrm{~kJ} \cdot \mathrm{mol}^{-1}$ (McInerney et al., 2009; Schink and Stams, 2013). Moreover, the available free energy must be shared by the different organisms (Schink, 1997). The energy conservation mechanisms used to establish and maintain a syntrophic lifestyle when the thermodynamic driving force is very low are not completely understood and have been subject of research over the last years. Research in anaerobic microorganisms has increased our understanding of the metabolic capabilities of bacteria and archaea 
and helped to elucidate mechanisms such as reverse electron transport, flavin based electron bifurcation and its reversal, electron confurcation (Li et al., 2008; McInerney et al., 2011; Buckel and Thauer, 2013; Schink, 2015).

\section{Reverse electron transport (RET)}

Propionate and butyrate are important intermediates in the degradation of organic matter to methane (Dong et al., 1994; Schmidt and Ahring, 1995; Kleerebezem and Stams, 2000). Although propionate can be dismutated to acetate and butyrate by Smithella propionica (de Bok et al., 2001), the most common biochemical pathways of syntrophic propionate and butyrate oxidation are methylmalonyl-CoA pathway (MMC) and beta-oxidation, respectively (Figure 1.2). In MMC reducing equivalents are formed in the oxidation of succinate to fumarate, malate to oxaloacetate, and pyruvate to acetyl-CoA $+\mathrm{CO}_{2}$. These equivalents are released at the level of $\mathrm{FADH}_{2}$ (enzyme bound), $\mathrm{NADH}$ and $\mathrm{Fd}_{\text {red }}$, respectively. In beta-oxidation the oxidation of hydroxybutyryl-CoA is $\mathrm{NADH}$-dependent, but the oxidation of butyryl-CoA to crotonyl-CoA is the energetically most difficult step in butyrate conversion due to the high redox potential of this electron pair $\left(\mathrm{E}^{\circ \prime}=-125 \mathrm{mV}\right)$ (Losey et al., 2017), as the oxidation of succinate is the most energy-consuming step in MMC (Stams and Plugge, 2009). NADH oxidation coupled to hydrogen/formate formation is energetically feasible when the concentration of these compounds is kept low by methanogens. However, the oxidation of $\mathrm{FADH}_{2} / \mathrm{FAD}\left(\mathrm{E}^{\circ}=-220 \mathrm{mV}\right)$ would require much lower hydrogen or formate concentrations than those that can be achieved by methanogens (Stams et al., 2005). It has been estimated that to couple this step to proton or $\mathrm{CO}_{2}$ reduction would require a partial pressure of hydrogen $\left(p \mathrm{H}_{2}\right)$ of $10^{-10}$ $\mathrm{Pa}$ (Schink, 1997); while the minimal $\mathrm{pH}_{2}$ that methanogens can maintain is between 1 to $10 \mathrm{~Pa}$ (Thauer et al., 2008). Therefore, by means of a reverse electron flow mechanism, acetogenic bacteria invest metabolic energy to make protons accessible to accept electrons from $\mathrm{FADH}_{2}$. This mechanism is known as reverse electron transport (RET) (Schink, 1997; van Kuijk et al., 1998b; Schink and Stams, 2013).

During RET energy is invested in the form of ATP to generate a proton gradient across the membrane which allows butyryl-CoA and succinate oxidation to proceed (Stams and Plugge, 2009). Several membrane-bound proteins like butyryl-CoA dehydrogenase, succinate dehydrogenases, Ech and Rnf to cytochromes and periplasmic formate dehydrogenases and hydrogenases, have been reported to be involved in RET (Müller et al., 2009; Sieber et al., 2012; Grein et al., 2013; Schmidt et al., 2013; Sieber et al., 2015). Therefore, reverse electron transport makes use of membrane-associated respiratory chains (Müller et al., 2010). However, the discovery in the last decade of soluble enzyme complexes that use the energy of a favourable redox reaction to drive an unfavourable redox reaction established the bases for the recognition of a third mechanism of biological energy conservation (McInerney et al., 2009; Stams and Plugge, 2009; Peters et al., 2016). 


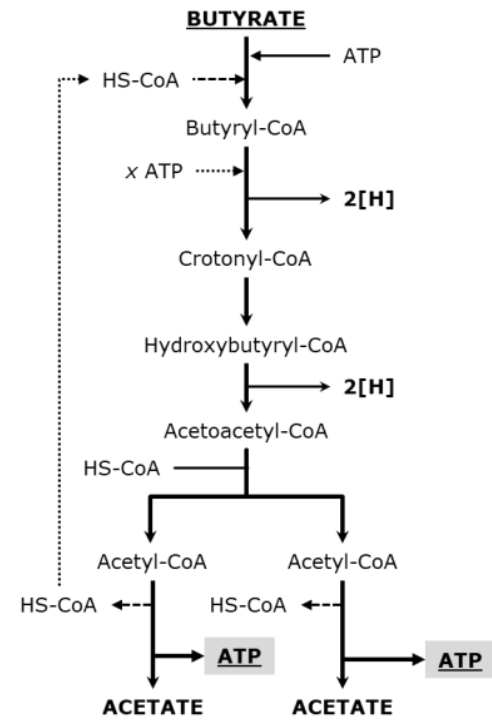

Beta-oxidation

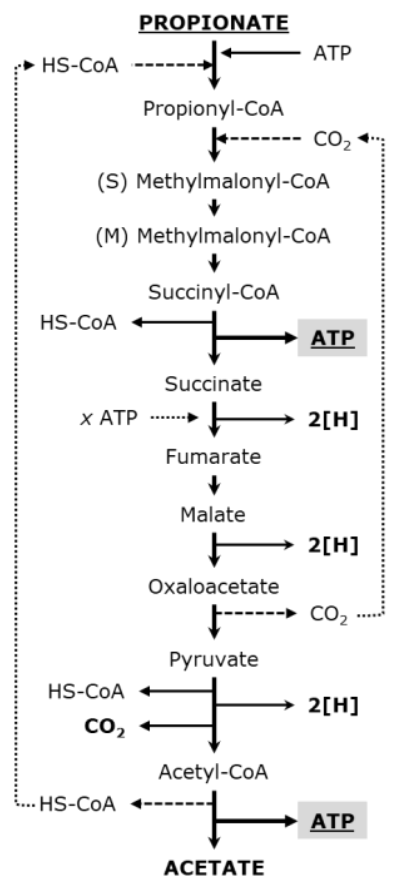

Methylmalonyl-CoA

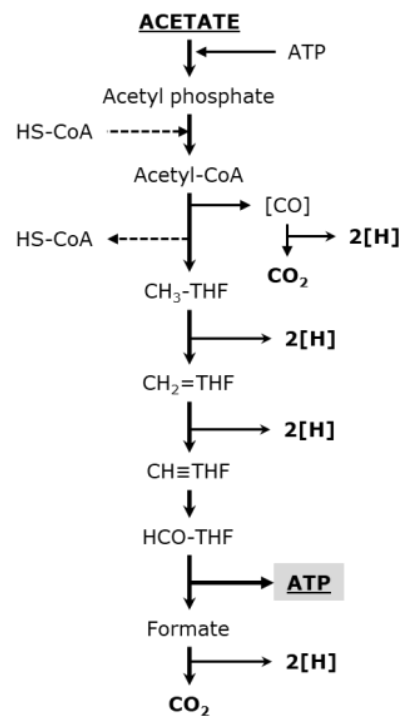

Wood-Ljungdahl

Figure 1.2. Metabolic pathways for acetate, propionate and butyrate conversion by bacteria that can grow in syntrophy with methanogens. Modified from (Worm et al. 2014)

\section{Electron bifurcation and electron confurcation}

Electron bifurcation is an energy conservation mechanism in which an endergonic reduction reaction is catalysed by coupling it to two exergonic oxidation reactions (Herrmann et al., 2008). Electron confurcation is the reversal of electron bifurcation (Buckel and Thauer, 2013; Schink, 2015). Hence, in electron bifurcation a pair of electrons is acquired at intermediate reduction potential (intermediate reducing power) and each electron is passed to a different acceptor, one with lower and the other with higher reducing power, leading to 'bifurcation'. While in electron confurcation a two-electron acceptor of intermediate reduction potential simultaneously accepts electrons from electron donors with more negative and more positive potentials (Figure 1.3) (Peters et al., 2016; Hoben et al., 2017).

The principle of electron bifurcation was originally proposed for a butyryl-CoA dehydrogenase/electron transferring flavoprotein complex (Bcd-Etf) in Clostridium kluyveri ( $\mathrm{Li}$ et al., 2008). Later, three more flavin-containing complexes from anaerobic bacteria and archaea were described: [FeFe]-hydrogenases (Hyd), transhydrogenases (NfnAB) and [NiFe]-hydrogenase/heterodisulfide reductases 
(MvhADG-HdrABC) (Schut and Adams, 2009; Kaster et al., 2011b; Huang et al., 2012; Buckel and Thauer, 2013). Up to date, at least seven types of reactions are known to be catalysed by bifurcating flavoenzymes (Peters et al., 2016; Zhang et al., 2017).
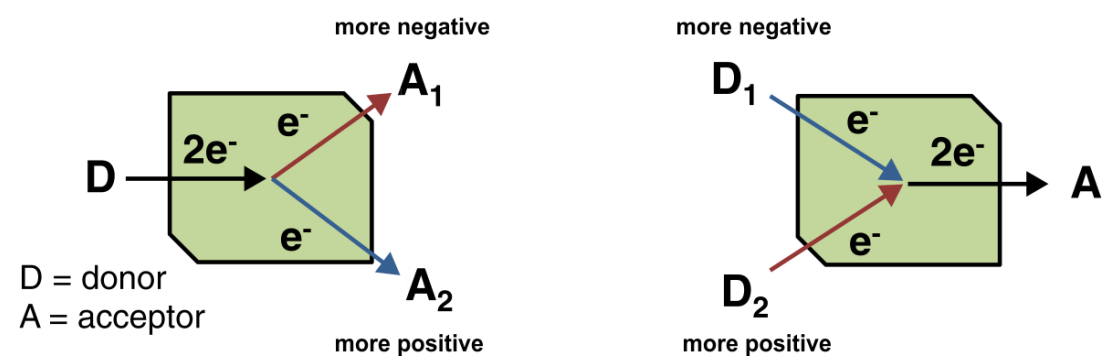

Figure 1.3. Scheme depicting electron transfer in bifurcating (left) and confurcating (right) complexes. In bifurcation a two-electron donor (D) of intermediate reduction potential $\left(\mathrm{E}^{\circ}\right)$ simultaneously provides electrons to electron acceptors with more negative $\left(\mathrm{A}_{1}\right)$ and more positive $\left(\mathrm{A}_{2}\right)$ potentials. While in confurcation a two-electron acceptor (A) of intermediate $\mathrm{E}^{\circ 1}$ simultaneously accepts electrons from electron donors with more negative (D1) and more positive (D2) $\mathrm{E}^{\circ}$. Modified from (Peters et al., 2016)

The discovery of the bifurcating complex Bcd-Etf in C. kluyveri opened the possibility and quest to find similar complexes that could perform the reverse during syntrophic fatty acid oxidation (Müller et al., 2009). After the description of the confurcating hydrogenase that couples the energetically unfavourable formation of hydrogen from $\mathrm{NADH}$ with the energetically favourable formation of hydrogen from reduced ferredoxin (Schut and Adams, 2009), homologs to this hydrogenase complex were found in Syntrophomonas wolfei and S. fumaroxidans genomes (Müller et al., 2010; Sieber et al., 2010). Currently confurcating hydrogenases are found in the genomes of all hydrogen-generating syntrophs described to date (Sieber et al., 2012; Worm et al., 2014). Electron bifurcation from formate has also been described (Costa et al., 2010; Costa et al., 2013b; Wang et al., 2013b)

Hydrogenotrophic methanogens use electron bifurcation as a primary energy coupling step in methanogenesis from $\mathrm{CO}_{2}$ (Costa et al., 2010; Kaster et al., 2011b). In the case of the hydrogenotrophic methanogenic pathway the bifurcation process involves a formylmethanofuran dehydrogenase (Fmd), an heterodisulfide reductase (Hdr) complex and an F420-non-reducing hydrogenase (Mvh) or a formate dehydrogenase $(\mathrm{Fdh})$ that connects the final step of methanogenesis to the initial reduction of $\mathrm{CO}_{2}$ (Lie et al., 2012; Costa and Leigh, 2014).

The details of how these flavoprotein complexes couple energetically downhill and uphill redox reactions are still under research, but the process is remarkable from 
both thermodynamic and kinetic perspectives (Lubner et al., 2017). How can proteins drive two electrons from a redox active donor onto two acceptors at very different potentials and distances? And how can this transaction be conducted without dissipating energy very much or violating the laws of thermodynamics? (Zhang et al., 2017). Moreover, it was proposed that bifurcation is mediated by a flavin, however there are numerous flavoenzymes that can carry two-electrons but cannot perform electron bifurcation (Garcia Costas et al., 2017; Hoben et al., 2017); it remains important to identify factors required for a flavin site to execute bifurcation. The number of genes encoding bifurcating/confurcating proteins found in many anaerobic microorganisms, indicates that the presently known flavin-based electronbifurcating enzyme complexes are only the tip of an iceberg.

\section{Anaerobic degradation of propionate}

\section{Syntrophy and sulfate reduction}

Propionate oxidation coupled to hydrogen or formate production is endergonic under standard conditions (Table 1.1). However, when propionate oxidation is coupled to methane production the conversion is energetically feasible (Eq. 2). The oxidation of propionate coupled to sulfate reduction is also an energy yielding reaction, Equation 5 :

$$
\begin{gathered}
4 \text { Propionate }^{-}+3 \mathrm{SO}_{4}^{-2} \rightarrow 4 \text { Acetate }^{-}+4 \mathrm{HCO}_{3}{ }^{-}+3 \mathrm{HS}^{-}+\mathrm{H}^{+} \\
\Delta \mathrm{G}^{\mathrm{o}}=-37.7 \mathrm{~kJ} / \mathrm{mol}
\end{gathered}
$$

Consequently, syntrophic fatty acid-degrading communities in anaerobic environments may be affected by the presence of sulfate. When sulfate is present, sulfate-reducing bacteria (SRB) compete with methanogens for hydrogen, formate and acetate, and with syntrophic methanogenic communities for substrates like propionate and butyrate (Muyzer and Stams, 2008). While many SRB can grow without sulfate and are engaged in syntrophic associations with methanogens, others lack this ability (Worm et al., 2014). For instance, Syntrophobacter species can use propionate in syntrophy with hydrogenotrophic methanogens, or alone if sulfate is available (Plugge et al., 2011; Liu and Conrad, 2017). Pelotomaculum species on the other hand do not possess the ability to couple propionate oxidation with sulfate reduction (Imachi et al., 2007).

Sulfate reduction is a respiratory process which includes oxidative phosphorylation through a still incompletely understood electron transfer pathway (Muyzer and Stams, 2008). Analysis of distinct organisms capable of sulfate reduction has helped to identify the minimal set of proteins required for this metabolic activity: Sulfate adenylyltransferase (Sat), APS reductase (AprAB), dissimilatory sulfite reductase 
(DsrAB) and DsrC and sulfate transporters (Pereira et al., 2011). Genomic studies have revealed that sulfate-reducing microorganisms use diverse processes for energy conservation involving membrane-based chemiosmotic or soluble flavin-based electron bifurcation mechanisms. Many of these proteins such as protontranslocating pyrophosphatase (HppA) or DsrC are also present in other non-sulfatereducing bacteria, whereas others like heterodisulfide reductases-related proteins are shared with methanogens (Grein et al., 2013).

One of the remaining important questions about sulfate reduction was the nature of the electron donors to the terminal reductases AprAB and DsrAB (Pereira et al., 2011). Recent studies have shown that a QmoABC membrane complex might serve as the physiological electron donor for AprAB, coupling the quinone-pool to sulfate reduction (Duarte et al., 2016). Moreover, the role of the DsrC trisulfide as the product of sulfite reduction by DsrAB and its link to energy conservation has also been reported recently (Santos et al., 2015). A glance into the versatile redox machinery of SRB involving membrane complexes such as Qrc, Qmo, DsrMKJOP, Nuo, Tmc, Hmc as well as cytoplasmic energy-conserving enzymes such as Hdr and Flox is helping to better understand the nature of prokaryotic energy metabolism (Grein et al., 2013; Rabus et al., 2015; Ramos et al., 2015; Santos et al., 2015). 


\section{Thesis outline}

The aim of this thesis is to gain insight into the molecular mechanisms used in anaerobic propionate degradation. In this thesis, the capacity of a model bacterium, Syntrophobacter fumaroxidans, to degrade propionate in syntrophy with methanogens, and in pure culture with different electron acceptors, was investigated. Special emphasis was given to the enzyme complexes used for energy conservation and interspecies electron transfer. Moreover, an in-depth analysis of the methanogenic partners and a sulfate-reducing partner was included in this work.

Chapter 2 addresses the fundamental and ecologically important question of why some microorganisms are able to engage in syntrophy with methanogens while others are not. A functional analysis of protein domains of a selected group of bacteria was performed. The bacterial strains were selected based on genome availability and their ability to grow on short chain fatty acids alone or in syntrophic association with methanogens. The research shows, at genomic level, the molecular mechanisms available in syntrophic bacteria that could facilitate syntrophic interactions with methanogens. The presence of periplasmic formate dehydrogenases and their maturation protein FdhE was found to be a typical difference between syntrophic and non-syntrophic butyrate and propionate degraders. Furthermore, a domain (CapA) putatively involved in capsule or biofilm production and another domain (FtsW/RodA/SpoVE) involved in cell division, shape-determination or sporulation seemed to be associated with the ability of syntrophic growth.

In Chapter 3 the metabolic flexibility of Syntrophobacter fumaroxidans to grow in syntrophy or as sulfate-reducing bacterium was assessed. The metabolic flexibility of sulfate-reducing bacteria to form syntrophic associations, despite their ability to reduce sulfate and oxidize fatty acids on their own, is an important topic that has been investigated to gain knowledge about the dynamics and resilience of anaerobic microbial communities. Perturbations in sulfidogenic pure cultures of $S$. fumaroxidans and in methanogenic cocultures of the bacteria with Methanospirillum hungatei were performed. Desulfovibrio desulfuricans was also used as an alternative syntrophic partner of $S$. fumaroxidans in sulfate rich environments. Growth of $D$. desulfuricans in the coculture with $S$. fumaroxidans, would only be possible if Syntrophobacter transferred electrons to $D$. desulfuricans via hydrogen or/and formate. Although growth of $D$. desulfuricans in the coculture was verified, it could not be clearly shown that $S$. fumaroxidans switched its metabolism from sulfidogenesis to syntrophy.

In Chapter 4 a proteomic comparison of five growing conditions of Syntrophobacter fumaroxidans is discussed. Proteomic data of $S$. fumaroxidans growing with propionate axenically with sulfate or fumarate, and in syntrophy with Methanospirillum hungatei, Methanobacterium formicicum or D. desulfuricans was 
analysed. Confurcating enzymes, formate dehydrogenases, hydrogenases, and other IET complexes and energy conservation mechanisms were scrutinized. Enzymes associated with sulfate reduction were also widely discussed. A brief proteomic analysis of the sulfate-reducing partner $D$. desulfuricans is also included in the chapter.

Chapter 5 completes and widens the proteomic analysis of the propionate-degrading syntrophic bacterium $S$. fumaroxidans by analysing the genome and proteome of the two methanogenic partners. The enzymes used in methanogenesis and energy conservation are discussed for $M$. hungatei and $M$. formicicum. Differences between the methanogens and among the cultured conditions (growth in $\mathrm{H}_{2} / \mathrm{CO}_{2}$, formate and in syntrophy with $S$. fumaroxidans) are described in detail. $M$. formicicum uses a $\mathrm{F}_{420}$-non-reducing hydrogenase (MvhADG) for bifurcation with the formylmethanofuran dehydrogenase (Fmd) and heterodisulfide reductase (Hdr). $M$. hungatei on the other hand employs an $\mathrm{F}_{420}$-reducing hydrogenase (FrhADGB). Differential production of enzymes involved in the methanogenic pathway as well as in diverse extracellular structures such as archaellum and pili are described in the analysis. Although both methanogens can grow on hydrogen and formate, the mechanisms available in their genome and the produced proteins, point to the use of hydrogen, in $M$. formicicum, and of formate, in M. hungatei, as electron carriers in their metabolism.

Throughout the thesis it is emphasized that formate and hydrogen are important intermediates in the anaerobic degradation of organic matter for which different microbes compete. In Chapter 6 the performance and robustness to high loading tests of an anaerobic membrane bioreactor (AnMBR) was evaluated. Considering that an increase in hydrogen might be useful to predict disturbances between fermentative processes and methanogenesis, we investigated if monitoring hydrogen can be used as an early warning indicator of process instability. The study analysed microbial community composition with next-generation sequencing techniques., process parameters and performance during the start-up and stable operation of a mesophilic AnMBR treating pot ale, as well as the resilience of the bioreactor and its biomass to overloading events.

Chapter 7 summarizes the findings of this thesis, discusses the outcome in a broader setting and provides future perspectives for research. The molecular mechanisms used by bacteria and archaea in syntrophy, methanogenesis and sulfate reduction are discussed in an integrative way. 
"ipse se nihil scire id unum sciat" P.? S.? 
N

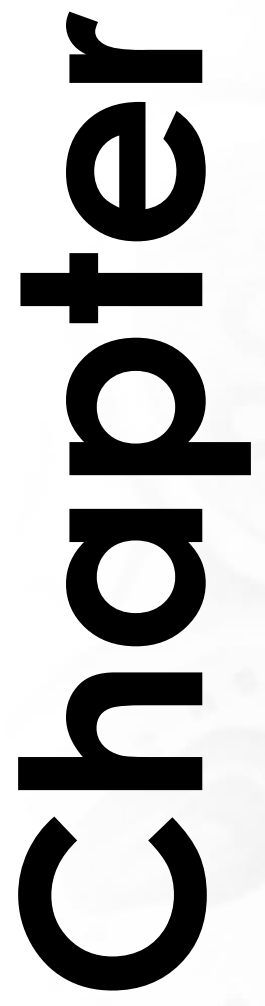




\section{CHAPTER 2}

\section{A genomic comparison of syntrophic and non- syntrophic butyrate- and propionate- degrading bacteria points to a key role of formate in syntrophy}

This chapter is adapted from Worm P., Koehorst J.J., Visser M., Sedano-Núñez V.T., Schaap P.J., Plugge C.M., Sousa D.Z. and Stams A.J.M. (2014) A genomic view on syntrophic versus non-syntrophic lifestyle in anaerobic fatty acid degrading communities. 1837:12, 2004-2016 


\begin{abstract}
In sulfate-reducing and methanogenic environments complex biopolymers are hydrolysed and degraded by fermentative microorganisms that produce hydrogen, carbon dioxide and short chain fatty acids. Degradation of short chain fatty acids can be coupled to methanogenesis or to sulfate reduction. Here we study from a genome perspective why some of these microorganisms are able to grow in syntrophy with methanogens and others are not. Bacterial strains were selected based on genome availability and upon their ability to grow on short chain fatty acids alone or in syntrophic association with methanogens. Systematic functional domain profiling allowed us to gain insight on this fundamental and ecologically important question. Extra-cytoplasmic formate dehydrogenases (InterPro domain number; IPR006443), including their maturation protein FdhE (IPR024064 and IPR006452) is a typical difference between syntrophic and non-syntrophic butyrate and propionate degraders. This also implies that formate is an important electron carrier in syntrophic butyrate and propionate degradation. Furthermore, two domains with a currently unknown function seem to be associated with the ability of syntrophic growth. One is putatively involved in capsule or biofilm production (IPR019079) and a second in cell division, shape-determination or sporulation (IPR018365). The sulfate reducing bacteria Desulfobacterium autotrophicum HRM2, Desulfomonile tiedjei and Desulfosporosinus meridiei were never reported for syntrophic growth, but all crucial domains were found in their genomes, which suggests their possible ability to grow in syntrophic association with methanogens. In addition, profiling domains involved in electron transfer mechanisms revealed the important role of the Rnf-complex and the formate transporter in syntrophy, and indicates that DUF224 may have a role in electron transfer in bacteria other than Syntrophomonas wolfei as well.
\end{abstract}

Keywords: Syntrophy, propionate, butyrate, formate, interspecies electron transfer, functional profiling. 


\section{Introduction}

Environments with a low redox potential are abundantly present on earth, especially in the deeper zones of marine and freshwater sediments. The low redox potential is created by the depletion of oxygen and the formation of hydrogen sulfide in the anaerobic degradation of organic matter. In the decomposition of sulfur-containing organic compounds such as the amino acids (cysteine and methionine) and cofactors (biotin and thiamine) hydrogen sulfide is released. Additionally, hydrogen sulfide is formed by anaerobic microorganisms that respire with sulfate or other sulfur compounds, such as thiosulfate and elemental sulfur. This respiratory type of sulfidogenesis is quantitatively most important (Blank, 2009; Shao et al., 2010; Offre et al., 2013).

Respiratory sulfate reduction is an important process in nature, especially in marine sediments where the sulfate concentration is high (about $20 \mathrm{mM}$ ) (Muyzer and Stams, 2008). In freshwater environments that are generally low in sulfate, sulfate reduction does not play an important role unless hydrogen sulfide is rapidly oxidized by sulfide-oxidizing microbes (Lovley and Klug, 1983; Luther et al., 2011). In sulfatedepleted anoxic environments methanogenesis is the most abundant process (Laanbroek et al., 1982), (Stams and Plugge, 2009). Interestingly, in marine environments methanogenesis occurs as well, especially in zones where the available sulfate is not sufficient to degrade organic matter (Ferry and Lessner, 2008). In both marine and freshwater environments microbes involved in sulfate-reduction and methanogenesis interact strongly with each other, and this interaction is strongly depending on the availability of sulfate. Generally, sulfate reduction is favoured over methanogenesis when sufficient sulfate is present (Muyzer and Stams, 2008; Stams and Plugge, 2009).

In sulfate-reducing and methanogenic environments organic material is degraded in a cascade process. Complex biopolymers are first hydrolysed and degraded by fermentative microorganisms that produce hydrogen, carbon dioxide and organic compounds, typically organic acids (butyrate, propionate, acetate and formate) as products. In sulfate-reducing environments these compounds are the common substrates for sulfate-reducing microorganisms. Phylogenetically and physiologically sulfate reducing microorganisms are very diverse (Muyzer and Stams, 2008). Phylogenetically they occur in the bacterial and archaeal domain of life. Some sulfate reducers have the ability to grow autotrophically with $\mathrm{H}_{2}$ and sulfate as energy substrates. Often these autotrophs are the sulfate reducers that are also able to degrade acetate completely to $\mathrm{CO}_{2}$, employing the reversible Wood-Ljungdahl pathway for acetate degradation and acetate formation (Hansen, 1994).

In methanogenic environments, methanogens use $\mathrm{H}_{2} / \mathrm{CO}_{2}$, formate and acetate as the main substrates (Liu and Whitman, 2008). Methanogenic archaea belong to 
different phylotypes. The ability to use acetate is restricted to archaea belonging to the order Methanosarcinales, with Methanosarcina and Methanosaeta as important genera. The ability to grow with $\mathrm{H}_{2} / \mathrm{CO}_{2}$ and formate occurs in most of the currently described orders of methanogens (Liu and Whitman, 2008). Higher organic compounds such as propionate and butyrate, that are typical intermediates in methanogenic environments, are not degraded by methanogens. Therefore, acetogenic bacteria are required to degrade such compounds to the methanogenic substrates acetate, formate and $\mathrm{H}_{2} / \mathrm{CO}_{2}$ (McInerney et al., 2008; Stams and Plugge, 2009). For thermodynamic reasons such bacteria can only degrade propionate and butyrate when the products are efficiently taken away by methanogens. Thus, these acetogenic bacteria grow in obligate syntrophy with methanogens. The methanogenic substrates acetate and formate may be degraded by syntrophic communities as well (Dolfing et al., 2008; Hattori, 2008). Syntrophic acetate degradation especially occurs under conditions at which the activity of acetoclastic methanogens is low such as a high temperature and high levels of ammonium (Hattori, 2008).

Though the basic concepts of sulfate reduction and methanogenesis are clear, it is not very clear how sulfate-reducing and methanogenic communities in freshwater and marine sediments are responding to changes in the sulfate availability. The metabolic flexibility of sulfate reducing bacteria has been addressed recently (Plugge et al., 2010; Plugge et al., 2011; Meyer et al., 2013). Several sulfate reducers are able to grow acetogenically in syntrophic association with methanogens which is for instance the case for Syntrophobacter fumaroxidans growing with propionate. Nevertheless, not all sulfate reducers possess the ability to switch from a sulfatedependent lifestyle to a syntrophic lifestyle. For instance, Desulfobulbus propionicus is a bacterium that grows with propionate and sulfate, but it is not able to grow with propionate in syntrophy with methanogens. Similarly, the thermophilic sulfate reducer Desulfotomaculum kuznetsovii is able to degrade propionate with sulfate, but it is not able to grow in syntrophy with methanogens, while the phylogenetically closely related non-sulfate-reducing bacterium Pelotomaculum thermopropionicum grows with propionate in syntrophy with methanogens (Imachi et al., 2002).

This study focusses on syntrophic degradation of short chain fatty acids (SCFA) such as butyrate, propionate and acetate. In contrast to syntrophic degradation of ethanol and lactate, syntrophic SCFA degradation occurs at the limit of what is thermodynamically possible and requires at least one step with reversed electron transport (Schink, 1997). Here we address a fundamental and ecologically important question: "what are the key properties that make a SCFA degrading bacterium able to grow in syntrophy with methanogens and another not". The availability of genome sequences of bacteria that can and bacteria that cannot grow with SCFA in syntrophic association may allow to identify key genes in syntrophy. 


\section{Microbial functions required for syntrophic growth}

\section{Functional profiling strategies}

Bacterial strains were selected based on genome availability, and ability to grow on short chain fatty acids syntrophically or not. Sulfate reducers that grow on short chain fatty acids, whose genomes are available and currently have not been tested for syntrophic growth were included in our analysis (Table 2.1). Correct codon usage of sequences coding for selenocysteine-containing formate dehydrogenases and hydrogenases was verified. Our strategy was to compare first bacteria that degrade propionate and butyrate, and then to identify if similarities can also be found in acetate degraders. Functional domain profiles were obtained with InterProScan 5 (version 5RC7, 27th January 2014). To get more insight into microbial functions required for syntrophic growth, domain based functional profiles of five butyrate and or/ propionate-degrading syntrophs were compared with the non-syntrophs Desulfobulbus propionicus and Desulfotomaculum kuznetsovii. Domains only present in syntrophs are listed in Table 2.1. Genomes of sulfate reducers that degrade butyrate and/or propionate, but were never tested for syntrophy, were screened for these domains (Table 2.1).

Functional domains assigned to proteins involved in electron transport were separately analysed. Domains that were unique for each protein were selected. Genomes of short chain fatty acid degrading syntrophs, non-syntrophs and sulfate reducers that never have been tested for syntrophy were screened for these domains (Table 2.2). Electron transport mechanisms in short chain fatty acid degrading syntrophs and non-syntrophs were predicted from their genomes by using the tools described below.

\section{Electron transfer complexes predicted from genome analysis}

Gene analysis started with automatic annotations of genomes from DOE-Joined Genome Institute (Markowitz et al., Version 4.2. November 2013). NCBI-pBLAST analysis with sequences from biochemically confirmed active subunits, was used to indicate the presence of gene clusters coding for formate dehydrogenases, hydrogenases, Electron transfer flavoprotein (Etf) and Rnf complexes in the genomes of Syntrophomonas wolfei, Syntrophus aciditrophicus, Syntrophothermus lipocalidus, Syntrophobacter fumaroxidans, Pelotomaculum thermopropionicum, Desulfotomaculum kuznetsovii, and Desulfobulbus propionicus. N-terminal amino acid sequences that corresponded to formate dehydrogenase 1 and -2 of $S$. fumaroxidans were used to find the gene clusters that code for these enzymes. To identify cofactor binding motifs, transmembrane helices, and twin-arginine translocation motifs in the N-terminus we used the Pfam protein families database version 27.0 (March 2013) (Punta et al., 2012), TMHMM Server v. 2.0 (Moller et al., 
2001) and the TatP 1.0 Server (Bendtsen et al., 2005) respectively. RNA loop predictions with Mfold version 3.2. were used to predict incorporation of selenocysteine (Mathews et al., 1999; Zuker, 2003). We compared the predicted RNA loop in the 50-100 bp region downstream of the UGA-codon with the consensus loop by (Zhang and Gladyshev, 2005). Sequences with similarity to iron-only or [FeFe]hydrogenases, were manually analysed for the presence of conserved H-cluster residues (Stothard, 2000). Bifurcation of electrons can occur via FAD, without the presence of iron-sulfur clusters (Buckel and Thauer, 2013). When a FAD binding domain was predicted by Pfam we propose that electrons from reduced ferredoxin and NADH can confurcate. In some cases, also an NADH binding site and/or iron sulphur cluster binding motifs were found with Pfam. Cofactor binding to NADH: ubiquinone oxidoreductase subunits in bacteria as listed by Yano and co-workers (Yano, 2002) was predicted based on domain similarity as determined by Pfam. We predict that enzyme complexes with an NADH binding domain, iron-sulfur clusters and a domain binding $\mathrm{Mo} / \mathrm{W}$, Se or hydrogen and not necessarily flavin, might have electron confurcating functions. Iron-only hydrogenases ([Fe]-hydrogenases) do not contain Fe-S clusters nor $\mathrm{Ni}$ or $\mathrm{Fe}$ and were initially referred to as "metal-free" hydrogenases. They are present mainly in methanogens, belong to a phylogenetically distinct class and their function in bacteria is not clear (Vignais and Billoud, 2007).

\section{Domain based genome comparison of syntrophic and non-syntrophic propionate and/or butyrate degraders}

Six domains are present in the genomes of all analysed butyrate and/or propionate degrading syntrophs and not in non-syntrophs (Table 2.1). Domain "IPR006443" is exclusively present in the extra-cytoplasmic formate dehydrogenase (FDH) alpha subunit. Domains "IPR024064 and IPR006452" both belong to FdhE. The gene $f d h \mathrm{E}$ in Escherichia coli is required for maturation of the membrane bound FDH-complex (Schlindwein et al., 1990). The fact that extra-cytoplasmic formate dehydrogenases are only present in syntrophs and not in non-syntrophs strongly indicates that extracytoplasmic formate production is essential for syntrophic propionate and butyrate oxidation, and that formate plays a major role in interspecies electron transfer. The redox potential of the couple proton / hydrogen $\left(\mathrm{E}^{0}=-414 \mathrm{mV}\right)$ is slightly higher than the redox potential of the couple $\mathrm{CO}_{2} /$ formate $(-432 \mathrm{mV})$. The preference in syntrophic fatty acid-degrading communities has not been clear thus far, but a syntrophic relationship in which both hydrogen and formate can be transferred would be more flexible than when only hydrogen is transferred (Sieber et al., 2014). Moreover, multiple studies indicate that interspecies formate transfer is of significant importance in syntrophic degradation of butyrate and propionate. For example, Syntrophobacter fumaroxidans and Syntrophospora bryantii oxidize propionate and butyrate, respectively, in syntrophy with hydrogen and formateusing methanogens such as Methanospirillum hungatei and Methanobacterium 
formicicum, but not with the hydrogen only-using Methanobrevibacter arboriphilus (Jackson et al., 1999). In analogy with this, S. wolfei oxidizes butyrate faster with the formate and hydrogen-using $M$. hungatei than with the hydrogen-only using $M$. arboriphilus (McInerney et al., 1981). The importance of formate transfer in S. wolfei cocultures is supported further by the observed involvement of an extra-cytoplasmic formate dehydrogenase in the final reduction of $\mathrm{CO}_{2}$ with electrons generated by the butyryl-CoA to crotonyl-CoA conversion (Schmidt et al., 2013). Moreover, this extracytoplasmic formate dehydrogenase was more expressed during syntrophic growth compared to axenic growth (Schmidt et al., 2013).

Domain "IPR019079", named CapA, was found in genomes of all short chain fatty acid degrading syntrophs (including acetate oxidizers, data not shown) and was not present in the genomes of the two non-syntrophs (Table 2.1). CapA is part of a membrane bound complex that synthesizes poly- $\gamma$-glutamate to form a capsule or biofilm in Bacillus subtilis, B. anthracis, Staphylococcus epidermidis and Fusobacterium nucleatum (Candela and Fouet, 2006; Morikawa et al., 2006; Candela et al., 2009). The presence of this domain in SCFA degrading bacteria may contribute to the formation of exopolymeric substances that may facilitate syntrophic growth. Domain "IPR018365" is present in FtsW, RodA, SpoVE, that are membrane integrated proteins involved in cell division, shape-determination and sporulation in Escherichia coli and Bacillus subtilis (Ikeda et al., 1989; Joris et al., 1990; Mohammadi et al., 2014). What the exact function of this domain is in syntrophic butyrate and propionate degraders is unclear. The domain "IPR020539" that seems exclusively present in syntrophs in our analysis belongs to the protein Ribonuclease $\mathrm{P}$ which removes extra residues at the 5'- side from precursor tRNA, resulting in mature tRNA. However, what its function could be in syntrophic growth is unclear, but just coincidence cannot be excluded. As can be seen from Table 2.1, only one copy of this domain is present in the genome of a syntrophic bacterium, whereas for the domains involved in periplasmic formate dehydrogenases, CapA-domains and Cell cycle FtsW / RodA / SpoVE- domains, more copies are present. Furthermore, domain co-occurrence suggests that $D$. autotrophicum HRM2, D. tiedjei and D. meridiei are able to adopt a syntrophic lifestyle on SCFA.

\section{Domain based functional profiling of electron transfer mechanisms}

For syntrophic butyrate and propionate degradation, electron transfer mechanisms are required to transfer electrons to the terminal acceptor, which can be sulfate during sulfidogenic lifestyle or protons and/or $\mathrm{CO}_{2}$ during syntrophic lifestyle. As the previous paragraph focussed on functional domains that are present in all syntrophic and not in non-syntrophic propionate and/or butyrate degraders, here we profile the functional domains involved in electron transfer mechanisms (Table 2.2). 


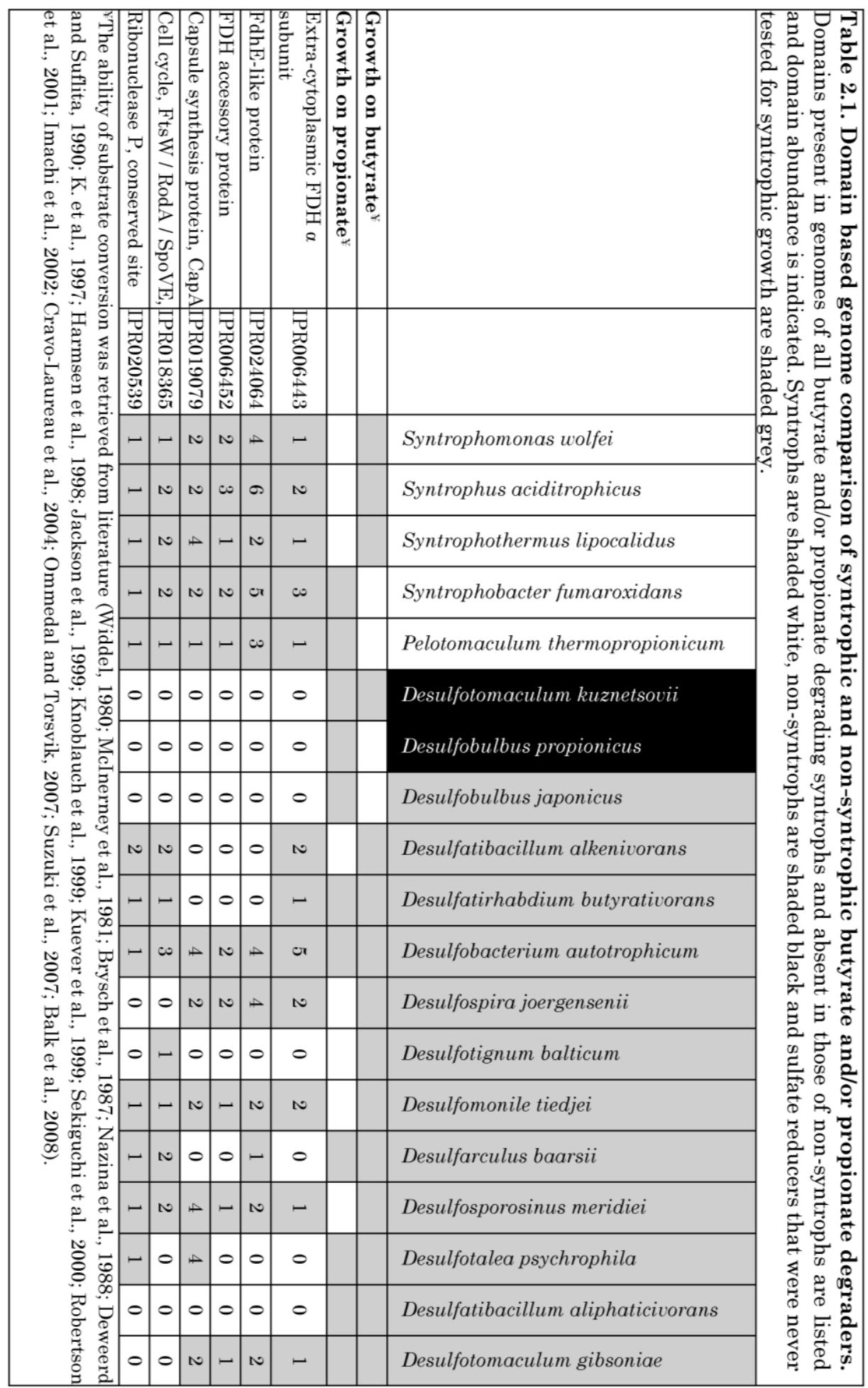




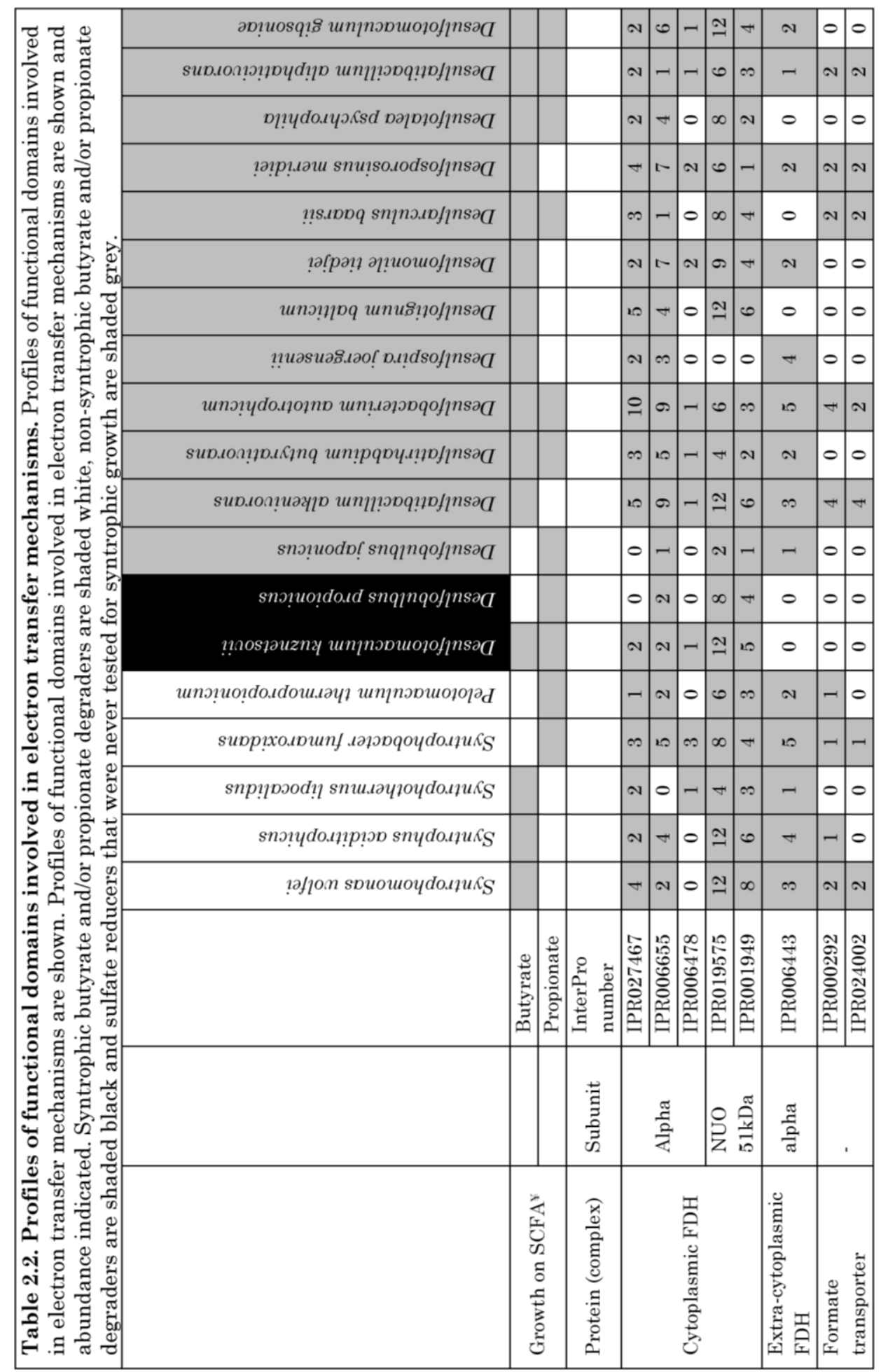




\begin{tabular}{|c|c|c|c|c|c|c|c|c|c|c|c|c|c|c|}
\hline 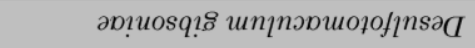 & $\sim$ & $n$ & $\infty$ & $\infty 0$ & $\infty \quad \sim$ & $\approx$ & 0 & 0 & 0.0 & 0.0 & 0 & -1 & $\vec{\infty}$ & $\infty$ \\
\hline 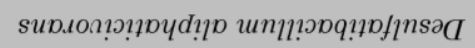 & 0 & 0 & 0 & 0.0 & $\infty$ & + & -1 & -10 & 0 & 0 & - & ง & 인 & $\bullet$ \\
\hline 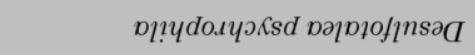 & + & 0 & 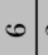 & ง & $\pm \cong$ & + & N & 0 & + & $+A$ & $\infty$ & ง & 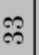 & $\infty$ \\
\hline 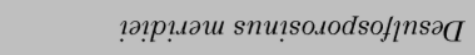 & $\infty$ & 10 & $\infty$ & $\infty$. & 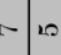 & 0 & 0 & $\infty 0$ & 0. & 0.0 & 0 & + & की & $\infty$ \\
\hline 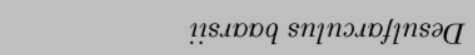 & -1 & -4 & 0 & 0.0 & N & $\because$ & 0 & -10 & ง & $-1-$ & N & $\infty$ & $\stackrel{\infty}{\infty}$ & $\sigma$ \\
\hline 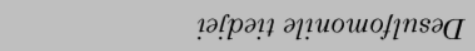 & -1 & -0 & $\infty$. & -0 & $\infty$ & $\Xi$ & -1 & -10 & ง & --1 & N & $\infty$ & ๑) & 10 \\
\hline 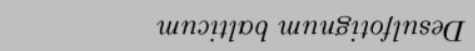 & N & ง & $\infty$ & - & • & 인 & 0 & -0 & 0. & ๑ & 0 & 10 & 范 & $\Xi$ \\
\hline 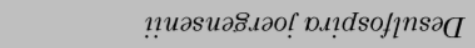 & - & - & $\infty$ & -0 & * & 잉 & -1 & N & 0. & --1 & ง & จ & $\vec{N}$ & $\bullet$ \\
\hline 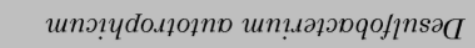 & - & 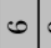 & $\infty$ & $\infty \%$ & $\stackrel{\infty}{\sim} \infty$ & ค & 20 & N & + & +4 & $\exists$ & 6 & B⿺辶冋 & 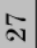 \\
\hline 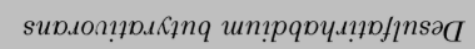 & 0 & 0 & 0 & 0. & $-N$ & $n$ & 0 & 0 & 0. & --1 & N & + & ละ & $\bullet$ \\
\hline 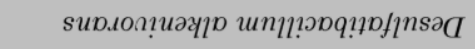 & 0 & 0 & 0 & $0: 2$ & $\stackrel{\sim}{\sim} \sigma$ & ลิ & -1 & 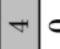 & 0.0 & \begin{tabular}{l|l}
$*$ \\
\end{tabular} & 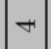 & 20 & \begin{tabular}{ll}
$\infty$ \\
\hdashline
\end{tabular} & $\stackrel{\theta}{-}$ \\
\hline snэ!uodp! snqlnqoflnsa & 0 & 0.0 & 0 & 0.1 & $r$ & 0 & 0 & $-c$ & 0. & 0.0 & 0 & 0 & $\infty$ & ○ \\
\hline snэฺนo! do.ıd snqlnqoflnsad & $\infty$ & $\infty .5$ & $\bullet$ & $\sim \curvearrowright$ & 0 & ง & 0 & 0 & ○ & 0.0 & 0 & N & $\simeq$ & 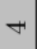 \\
\hline 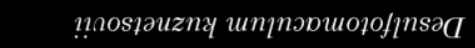 & $\infty$ & $\infty \%$ & $\stackrel{\mathcal{N}}{-}$ & +0 & 0 & 이 & 0 & 00 & 0 & 0 & 0 & -1 & $\stackrel{\circ}{\circ}$ & $\infty$ \\
\hline 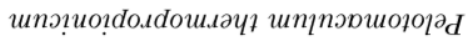 & + & 100 & 0 & $\infty 0$ & N & 6 & 0 & 0 & 0 & 0.0 & 0 & N & 9 & $\infty$ \\
\hline 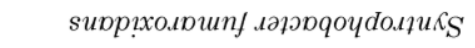 & $\infty$ & $\infty$ & $\bullet$ & a & $=0$ & 0 & -1 & -10 & ง & --1 & $\sim$ & - & ลิ & 10 \\
\hline 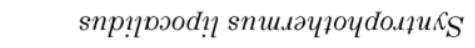 & $\infty$ & $\infty$ & $\infty$ & $\infty$. & $\infty \sim$ & 0 & 0 & 00 & 0 & 0.0 & 0 & -1 & $\therefore$ & - \\
\hline 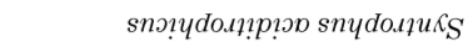 & -1 & -0 & $\infty$. & -0 & s & ง & -1 & -0 & ง & $-1-$ & N & - & 10 & - \\
\hline ?әдлоп sриошочдоцик & + & 10 & 0 & $\infty 0$ & 0 & ง & 0 & 0 & 0 & 0 & N & - & ○) & - \\
\hline & 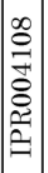 & 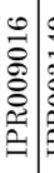 & 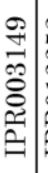 & 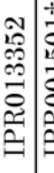 & 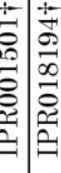 & 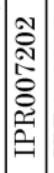 & 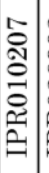 & 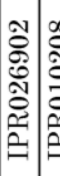 & 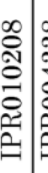 & 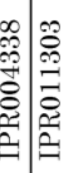 & 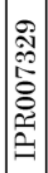 & 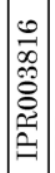 & 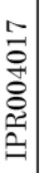 & 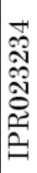 \\
\hline & & $\frac{\frac{\pi}{2}}{\frac{2}{\sigma}}$ & $\frac{\sigma}{2}$ & & & 蒫 & & 己ِّ & & क्षित्र & 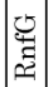 & & , & \\
\hline & & 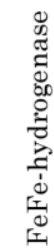 & : & & 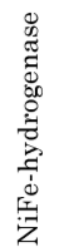 & \multicolumn{6}{|c|}{ 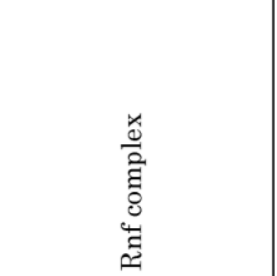 } & & 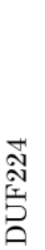 & \\
\hline
\end{tabular}




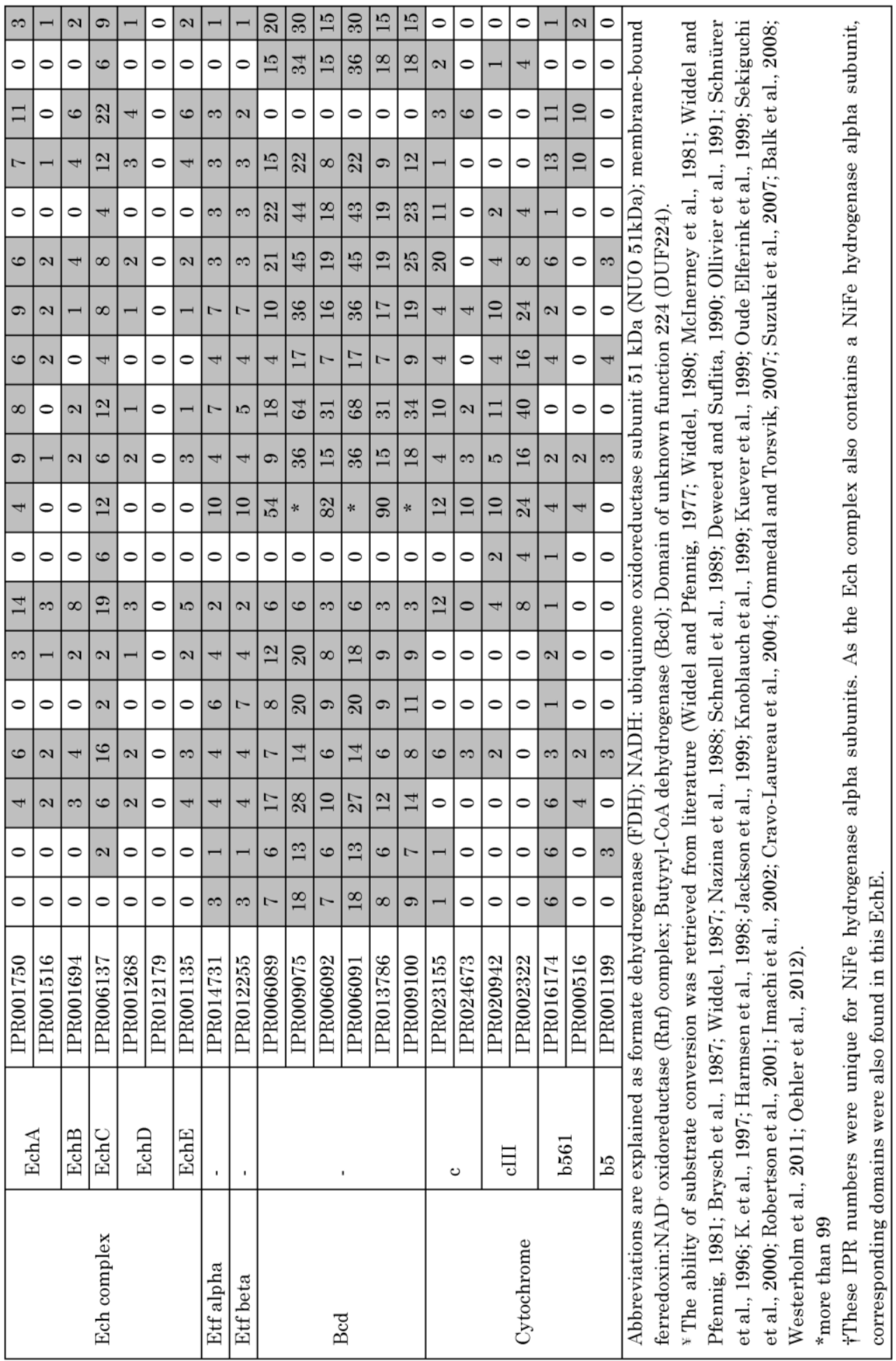


As can be seen from Table 2.2, cytoplasmic and extra-cytoplasmic formate dehydrogenases contain InterPro domains that are unique for each protein. "IPR006443" is only present in extra-cytoplasmic FDH's, not in cytoplasmic FDH's whereas "IPR027467", "IPR006655" and "IPR006478" of cytoplasmic FDH, are not present in extra-cytoplasmic FDH's. Domains of cytoplasmic FDH's are present in genomes of syntrophs and non-syntrophs, whereas the domain of extra-cytoplasmic FDH's is present only in syntrophs. Formate transporter linked domains are absent in genomes of non-syntrophs whereas they are present in a number of syntrophs. These observations again point to the importance of formate as interspecies electron carrier.

The membrane bound Rnf complex that can conserve energy by the reversible translocation of protons or sodium ions from ferredoxin oxidation with $\mathrm{NAD}^{+}$ (Tremblay et al., 2012) was not found in non-syntrophs but is present in several syntrophs. As syntrophs live at the limit of what is energetically possible (Schink, 1997; Scholten and Conrad, 2000; McInerney et al., 2007) they contain mechanisms to conserve energy from ferredoxin oxidation with $\mathrm{NAD}^{+}$. Furthermore, recently the domain with unknown function "DUF224" was shown to play a role in electron transport from an electron transfer flavoprotein (ETF) towards membrane-bound electron transfer components in S. wolfei (Schmidt et al., 2013). DUF224 is present in 18 genomes from which 17 also contain domains linked to ETF complexes. This indicates that DUF224 may have a role in electron transfer in bacteria other than $S$. wolfei as well.

\section{Energetics and metabolism of syntrophic butyrate and propionate degradation.}

\section{Energy conservation mechanisms}

For microbial maintenance and growth, the energy that is released from catabolic reactions has to be converted into energy that can be used to perform anabolic reactions. Therefore, energy is conserved as ATP by substrate level phosphorylation or via a proton or sodium gradient over the cytoplasmic membrane, termed electron transport phosphorylation. Membrane bound enzyme complexes are required to build a proton gradient over the membrane while other membrane bound enzyme complexes are required to use the proton gradient. The membrane bound enzyme complex ATP synthase can either use the proton gradient for ATP synthesis or ATP hydrolysis to build the proton gradient.

In addition to substrate level phosphorylation and the proton gradient over the cytoplasmic membrane, an only recently discovered process called flavin-based electron bifurcation has been considered as a third mechanism for energy conservation (Buckel and Thauer, 2013). In the last decade, several of such 
cytoplasmic bifurcation complexes were determined in Bacteria and Archaea (Li et al., 2008; Schut and Adams, 2009; Wang et al., 2010; Kaster et al., 2011b; Huang et al., 2012; Buckel and Thauer, 2013; Costa et al., 2013b; Wang et al., 2013a; Wang et al., 2013b). Instead of coupling two redox reactions, as is performed by commonly known redox proteins, bifurcation (and the reversed reaction termed confurcation) enzyme complexes couple three redox reactions. With this concept, energy that would otherwise have been lost can be conserved or endergonic reactions can be coupled to exergonic reactions and reducing equivalents that are generated can be re-oxidized efficiently. For instance, endergonic reduction of ferredoxin with NADH is coupled to the exergonic reduction of crotonyl-CoA to butyryl-CoA by the butyryl-CoA / electron transfer flavoprotein complex of Clostridium kluyveri (Li et al., 2008). Another example is the [FeFe]-hydrogenase complex of Thermotoga maritima that couples reversible ferredoxin reduction with hydrogen to $\mathrm{NAD}^{+}$reduction (Schut and Adams, 2009). In addition to cytoplasmic bifurcating enzyme complexes, membrane bound complexes (Rnf-complexes) were recently shown to conserve energy by the reversible translocation of protons or sodium from ferredoxin oxidation with $\mathrm{NAD}^{+}$(Biegel et al., 2011). The energy conserving hydrogenase (Ech) has a similar function, but performs the proton or sodium translocation by ferredoxin oxidation with hydrogen production (Hedderich and Forzi, 2005).

\section{Syntrophic butyrate degradation}

Butyrate oxidation coupled to hydrogen or formate production is endergonic under standard conditions. This is shown by the positive Gibbs free energy changes; +48 $\mathrm{kJ}$ and $+45 \mathrm{~kJ}$, respectively (Table 1.1). When butyrate oxidation is coupled to methane production the conversion is energetically feasible. To share this energy between the syntrophic butyrate oxidizer and the methanogen in such a manner that both organisms gain enough energy to grow, the hydrogen and formate concentrations have to be kept in a low range (Schink, 1997). Syntrophomonas wolfei, Syntrophus aciditrophicus and Syntrophothermus lipocalidus can couple butyrate oxidation to syntrophic growth with methanogens and cannot grow in pure culture with any electron acceptor (Beaty and McInerney, 1990; Jackson et al., 1999; Sekiguchi et al., 2000).

All known syntrophic butyrate degraders oxidize butyrate via the beta-oxidation pathway (Table 2.4, Figure 1.2) (McInerney et al., 2007; Stams and Plugge, 2009). This pathway includes two reactions that generate electron pairs and one reaction that generates ATP. This ATP partially has to be invested in the endergonic conversion of butyryl-CoA to crotonyl-CoA. The biochemical mechanism that enables investment of a fraction of ATP for the endergonic conversion of butyryl-CoA to crotonyl-CoA has recently been revealed in $S$. wolfei. Electrons that are generated by the conversion of butyryl-CoA to crotonyl-CoA travel via butyryl-CoA dehydrogenase (encoded by genes with locus tags Swol_1933 and Swol_2053), an electron transfer 
flavoprotein (encoded by Swol_0696-7) and a membrane anchored protein that was annotated as DUF224 (encoded by Swol_0698) to the menaquinone pool in the membrane. Oxidation of reduced menaquinone is then coupled to formate generation by a membrane anchored extra-cytoplasmic formate dehydrogenase (encoded by Swol_0797-800) (Schmidt et al., 2013). This reaction is driven by the proton motive force. The produced formate is used by the methanogen. The second reaction that generates electrons and protons is the conversion of hydroxybutyryl-CoA to acetoacetyl-CoA which is endergonic when coupled via $\mathrm{NAD}^{+}$to hydrogen or formate production. Most likely in $S$. wolfei this involves the [FeFe]-hydrogenase (encoded by Swol_1017-19) that forms a cytoplasmic complex with a formate dehydrogenase (Swol_0783-6) (Müller et al., 2009).

\section{Syntrophic propionate degradation}

Propionate oxidation coupled to hydrogen or formate production is endergonic under standard conditions. This is shown by the positive Gibbs free energy changes; +76 $\mathrm{kJ}$ and $+72 \mathrm{~kJ}$ respectively (Table 1.1). However, when propionate oxidation is coupled to methane production the conversion is energetically feasible. To share energy between the syntrophic propionate oxidizer and the methanogen in such a manner that both organisms gain enough energy to grow, the hydrogen and formate concentrations have to be kept in a low range (around $40 \mathrm{~Pa}$ ) (Schink, 1997). Smithella propionica, Syntrophobacter fumaroxidans and Pelotomaculum thermopropionicum are able to couple propionate oxidation to syntrophic growth with methanogens (Harmsen et al., 1998; Liu et al., 1999; Imachi et al., 2002; Kosaka et al., 2006). Smithella propionica degrades propionate via a dismutating pathway to acetate and butyrate, which is subsequently oxidized to acetate (de Bok et al., 2001). All other known syntrophic propionate-degrading bacteria use the methylmalonylCoA pathway to oxidize propionate to acetate and $\mathrm{CO}_{2}$ (Figure 1.2). In this pathway one ATP is formed via substrate level phosphorylation, 2/3 ATP have to be invested and three conversions in the methylmalonyl-CoA pathway generate each two electrons and two protons.

One of the reactions that generates two electrons and two protons is the endergonic oxidation of succinate to fumarate that requires investment of $2 / 3$ ATP (Schink, 1997). Van Kuijk et al. (1998) proposed that succinate oxidation could be coupled to extra-cytoplasmic hydrogen or formate formation via a menaquinone loop between a cytoplasmic oriented membrane-bound succinate dehydrogenase and a periplasmic oriented membrane bound hydrogenase or formate dehydrogenase (van Kuijk et al., 1998b) 


\begin{tabular}{|c|c|c|c|c|c|c|c|c|c|c|c|}
\hline 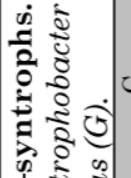 & . & $\cdot$. & & 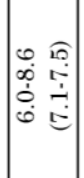 & 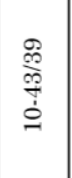 & 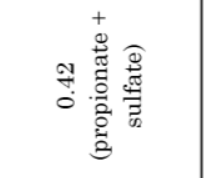 & 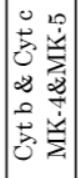 & $\sum_{i}^{0}$ & 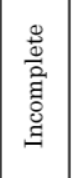 & 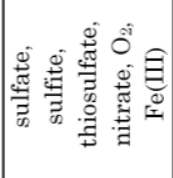 & 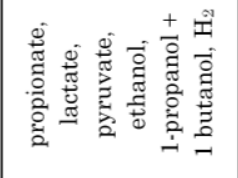 \\
\hline 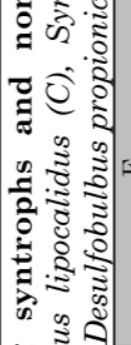 & $=$ & + & + & 言 & $\begin{array}{l}00 \\
0 \\
0 \\
0 \\
20 \\
\infty \\
0 \\
0 \\
10\end{array}$ & 豆 & 闩 & 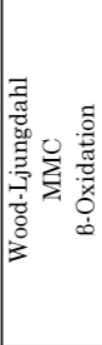 & 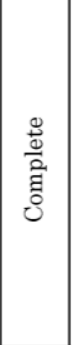 & 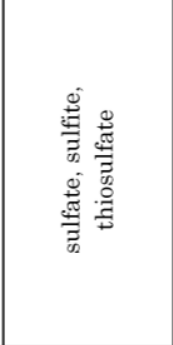 & 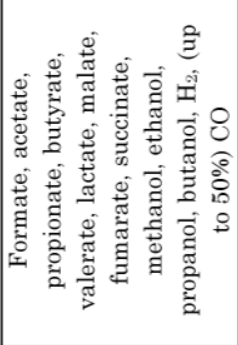 \\
\hline 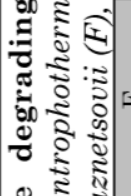 & 되 풍․ & . & $1+$ & 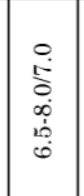 & 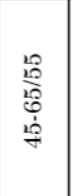 & 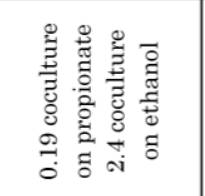 & 光 & 离 & 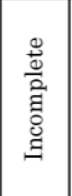 & 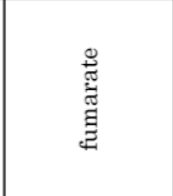 & 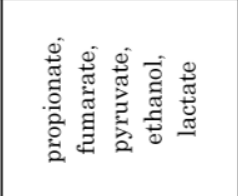 \\
\hline 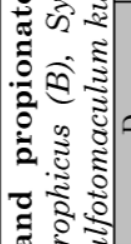 & D & & . & $\begin{array}{l}\frac{1}{0} \\
0 \\
0 \\
0 \\
0 \\
0\end{array}$ & 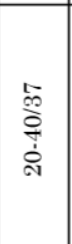 & 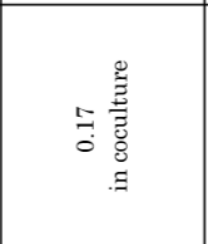 & 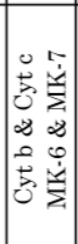 & 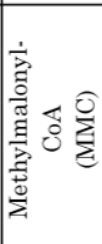 & 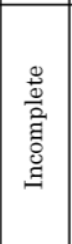 & 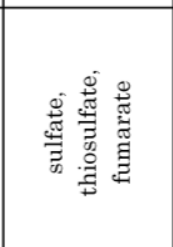 & 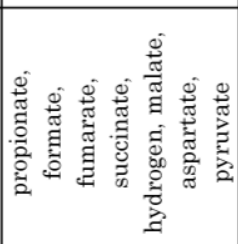 \\
\hline 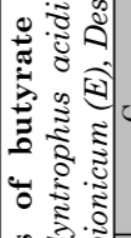 & ". & + & -1. & 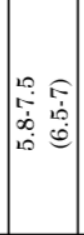 & 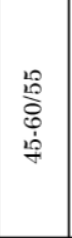 & 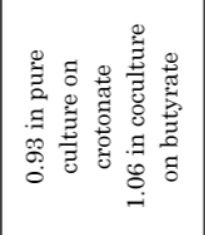 & 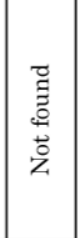 & 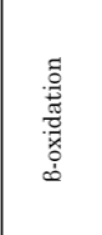 & 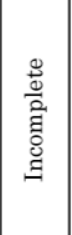 & そั & 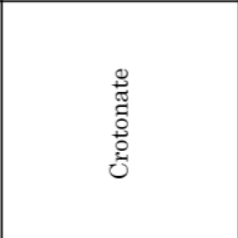 \\
\hline 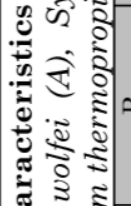 & $\infty$ & . & . & 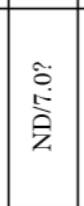 & 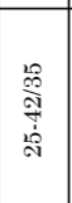 & 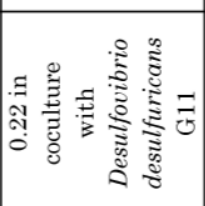 & $\stackrel{\Xi}{\Sigma}$ & 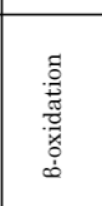 & 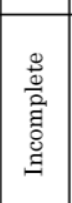 & 芒 & 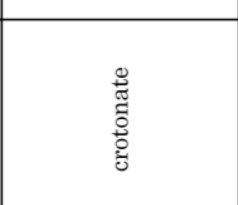 \\
\hline 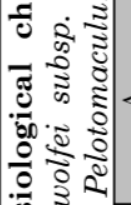 & $\varangle$ & $\cdot+$ &. & 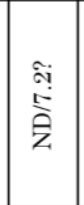 & 㕣 & 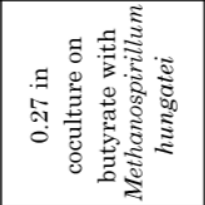 & 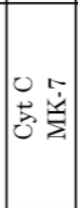 & 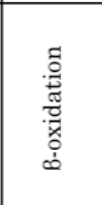 & 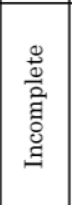 & 芩 & 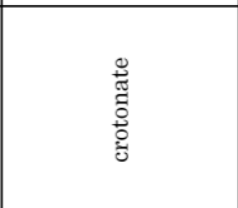 \\
\hline 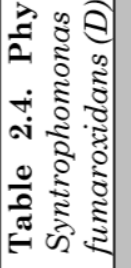 & 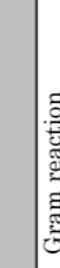 & |: & 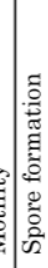 & 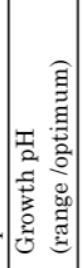 & 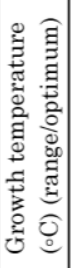 & 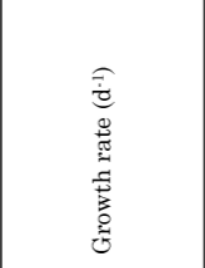 & 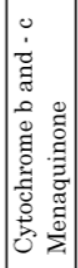 & 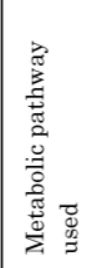 & 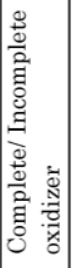 & 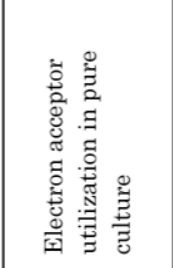 & 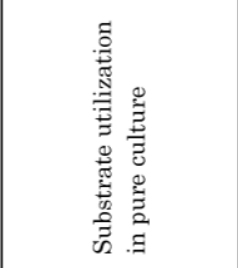 \\
\hline
\end{tabular}




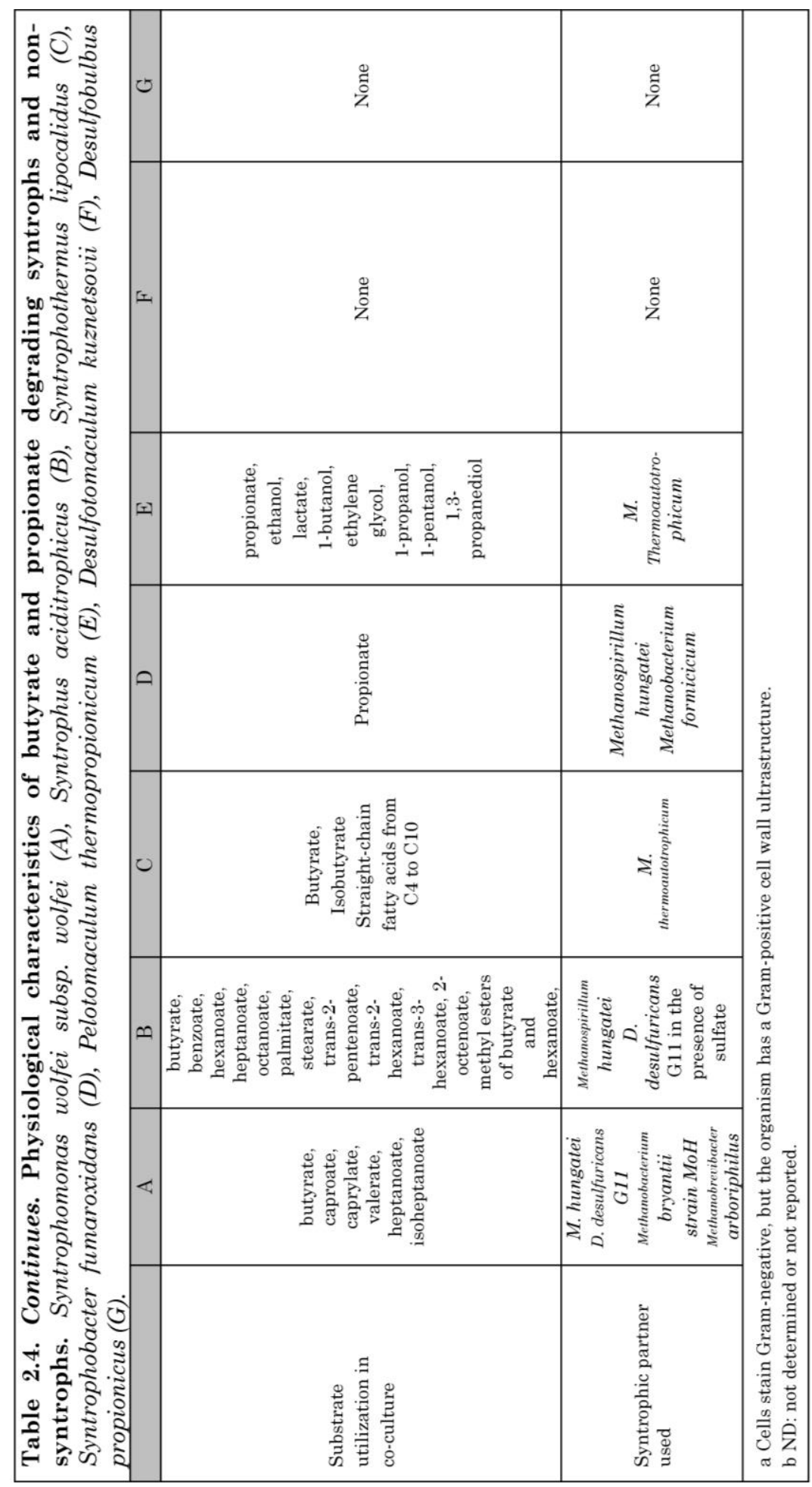


Genes coding for a periplasmic hydrogenase and three extra-cytoplasmic formate dehydrogenases were found in the genome of $S$. fumaroxidans (Müller et al., 2010). Especially the gene Sfum_1273-74 that codes for one of the periplasmic formate dehydrogenase alpha subunits is highly transcribed during syntrophic growth (Worm et al., 2011b) which suggests that succinate oxidation is coupled to formate production and indicates the importance of formate as an electron carrier in syntrophic propionate degradation. Also malate oxidation to oxaloacetate generates two electrons and two protons, which in $S$. fumaroxidans are coupled to $\mathrm{NAD}^{+}$ reduction by malate dehydrogenase (van Kuijk and Stams, 1996). To couple this to hydrogen production would require a hydrogen partial pressure of $10^{-8}$ atm that is lower than can be maintained by methanogens (Schink, 1997). The third reaction that generates electrons and protons is the conversion of pyruvate to acetyl-CoA and $\mathrm{CO}_{2}$ that can be coupled to ferredoxin reduction using the pyruvate:ferredoxin oxidoreductases (Chabriere et al., 1999). Genome analysis suggests that NADH generated from malate oxidation and reduced ferredoxin generated from pyruvate oxidation could be coupled to formate or hydrogen production by confurcating formate dehydrogenases and hydrogenases (Müller et al., 2010). Such a mechanism would use the energy that remains from ferredoxin oxidation with protons to allow the endergonic coupling of $\mathrm{NADH}$ oxidation to proton reduction. Formate dehydrogenases from $S$. fumaroxidans were studied for subunit-composition, enzyme activity, cofactor binding and direction of conversion. Formate dehydrogenase 1 contains W, Se, four $[2 \mathrm{Fe} 2 \mathrm{~S}]$, one $[4 \mathrm{Fe} 4 \mathrm{~S}]$ and is a hetero-trimer. Formate dehydrogenase 2 contains $\mathrm{W}$, Se, two [4Fe4S] and is heterodimer. Both enzymes oxidize formate with benzyl viologen and reduce $\mathrm{CO}_{2}$ with reduced methyl viologen. The purified enzyme was not able to reduce $\mathrm{NAD}^{+}$(de Bok et al., 2003). Whether these formate dehydrogenases can confurcate electrons from $\mathrm{NADH}$ and reduced ferredoxin to $\mathrm{CO}_{2}$ reduction, has never been tested.

\section{Syntrophic formate degradation}

Genome comparison pointed to the role of formate in syntrophic butyrate and propionate degradation. In the degradation of SCFA, formate and hydrogen play an important role as electron shuttling components. Interestingly syntrophic growth with formate occurs as well. Formate oxidation coupled to hydrogen is endergonic under standard conditions. This is shown by the Gibbs free energy change that is close to zero; $1.3 \mathrm{~kJ}$ (Table 1.1). However, when formate oxidation is coupled to methane production the conversion is energetically feasible. To share energy between the syntrophic formate oxidizer and the methanogen in such a manner that both organisms gain enough energy to grow, the hydrogen concentrations have to be kept in a low range (between 40 and $100 \mathrm{~Pa}$ ) (Dolfing et al., 2008). The thermophilic Moorella sp. strain AMP and mesophilic Desulfovibrio desulfuricans G11 are able to couple formate oxidation to syntrophic growth with methanogens that can only use 
hydrogen as electron donor (Dolfing et al., 2008). The electron transfer mechanism that allows syntrophic formate degradation is not known. Possibly an extracytoplasmic formate dehydrogenase is coupled to a membrane integrated, cytoplasmic oriented hydrogenase which generates a proton motive force that can be used or ATP synthesis (Dolfing et al., 2008). To what extend and in what types of anaerobic microbial environments syntrophic formate degradation can compete with formate degradation by methanogens is not known.

\section{Phylogeny of short chain fatty acid degraders does not predict syntrophy}

Syntrophic methanogenic growth on butyrate is performed by bacteria belonging to the Firmicutes (Syntrophomonas, Syntrophothermus, Thermosyntropha genera) and Deltaproteobacteria (Syntrophus aciditrophicus). Syntrophomonas is the best represented genus within syntrophic fatty-acid degraders (in terms of available isolates), with 11 species and/or subspecies described thus far (Sousa et al., 2009). Nevertheless, only the genome of $S$. wolfei subsp. wolfei has been sequenced (Sieber et al., 2010). Propionate can be syntrophically utilized by Pelotomaculum- and Syntrophobacter species (Stams et al., 2012). In addition, Smithella propionica can degrade propionate in syntrophy with methanogens (Liu et al., 1999). Syntrophobacter species can use propionate in syntrophy with hydrogenotrophic methanogens, or alone if sulfate is available in the environment (Plugge et al., 2011). Pelotomaculum species do not possess the ability to grow with propionate and sulfate. The genomes of Syntrophobacter fumaroxidans and Pelotomaculum thermopropionicum are available (Kosaka et al., 2008; Plugge et al., 2012).

Dissimilatory sulfate-reducing bacteria able to use fatty-acids are very diverse. Sulfate-reducing bacteria analysed in the scope of this study are distributed among Deltaproteobacteria and Firmicutes phyla. Desulfotomaculum species belong to Peptococcaceae family, the same family of the syntrophic Pelotomaculum species. Recently, it was shown that the genomes of $D$. kuznetsovii and P. thermopropionicum have a high similarity (Visser et al., 2013). The genes involved in propionate metabolism of these two strains were similar, but main differences were found in genes involved in the electron acceptor metabolism. Some Desulfotomaculum species - D. thermobenzoicum subsp. thermosyntrophicum and D. thermocisternum - were also shown to grow on propionate in syntrophy with a hydrogenotrophic methanogen (without sulfate) (Nilsen et al., 1996; Plugge et al., 2002).

The ability to grow in syntrophy is either first evolved and then lost or acquired by horizontal gene transfer from a syntroph to a non-syntroph. Multiple horizontal gene transfers of dissimilatory sulfite reductase genes $(d s r A B)$ in sulfate-reducing prokaryotes have been suggested by (Klein et al., 2001). These authors found that the topology of a tree based on a large fragment of the $d s r A B$ was inconsistent with the corresponding $16 \mathrm{~S}$ based tree. 


\section{Conclusions}

Systematic functional profiling of genomes shed light on the question: "what are the key properties that make that a SCFA degrading bacterium is able to grow in syntrophy with methanogens and another not". The presence or absence of extracytoplasmic formate dehydrogenases, including their maturation proteins is clearly a difference between syntrophic and non-syntrophic butyrate and/or propionate degraders. Together with transcription and proteomic studies that show an increase of extra-cytoplasmic formate dehydrogenase during syntrophic growth (Worm et al., 2011b; Schmidt et al., 2013), it seems evident that this enzyme is a key factor for syntrophic butyrate and propionate degradation. Moreover, this simultaneously suggests that formate is an important interspecies electron carrier in syntrophic butyrate and propionate degradation. This is supported by the presence of the formate transporter in several butyrate and propionate degrading syntrophs. Further biochemical examination and knock-out experiments of genes involved in formate transport and extra-cytoplasmic formate dehydrogenase activity and maturation would give more insight in the importance of this enzyme complex during syntrophy. Genetic manipulation protocols for SCFA degrading syntrophic bacteria have to be developed. Furthermore, the presence or absence of two domains, both linked to membrane integrated proteins with a currently unknown function in syntrophy, appear to make a difference as well. Both are membrane integrated proteins. One is putatively involved in capsule or biofilm formation and a second in cell division, shape-determination or sporulation. Capsule formation, cell division, shape-determination and sporulation by these bacteria during syntrophic growth could be assessed with microscopic techniques.

Sulfate reducing bacteria such as Desulfobacterium autotrophicum HRM2, Desulfomonile tiedjei and Desulfosporosinus meridiei were never tested for syntrophic growth, but all crucial domains discussed in this review were found in corresponding genomes, which suggests their possible ability to grow in syntrophic association with methanogens. In addition, profiling domains involved in electron transfer mechanisms revealed the important role of the Rnf-complex and the formate transporter in syntrophy, and indicates that DUF224 may have a role in electron transfer in bacteria other than $S$. wolfei as well. 


\section{Supporting information}

\section{A. Syntrophobacter fumaroxidans}

$\mathrm{H}^{+}+\mathrm{CO}_{2}$ Formate-

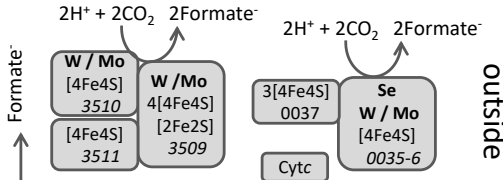

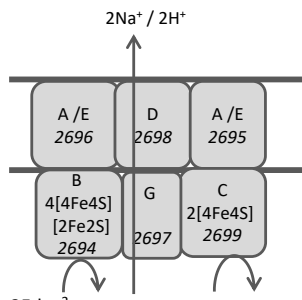

$2 \mathrm{Fd}_{\text {red }}{ }^{2-} 2 \mathrm{Fd}_{\mathrm{Ox}} \mathrm{NAD}+\mathrm{H}^{+} \quad \mathrm{NADH}^{+}$

Rnf-complex
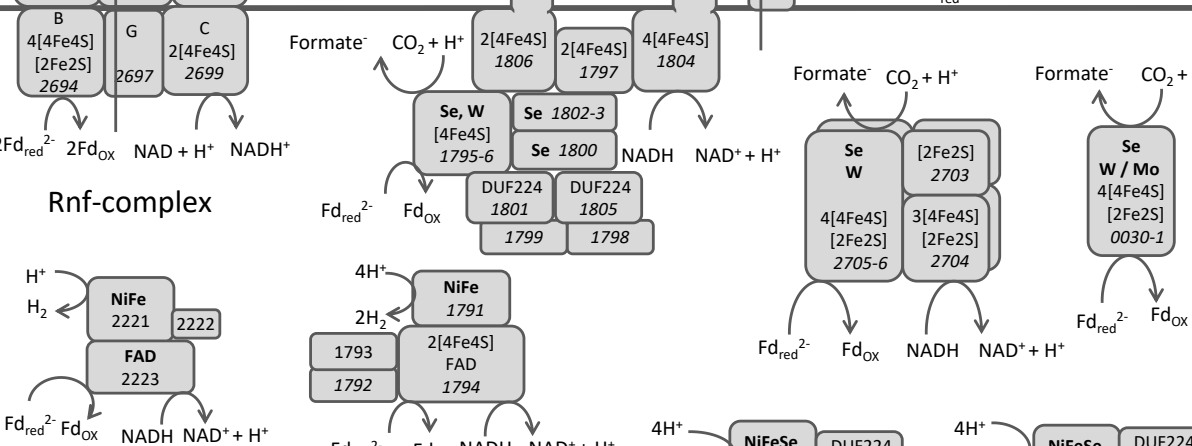

$\mathrm{Fd}_{\text {red }}^{2-} \mathrm{Fd}_{\mathrm{OX}} \mathrm{NADH} \mathrm{NAD}^{+}+\mathrm{H}^{+}$

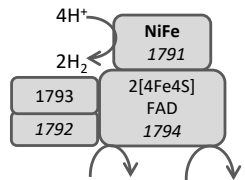

$$
\mathrm{Fd}_{\text {red }}{ }^{2-} \quad \mathrm{Fd}_{\mathrm{Ox}} \mathrm{NADH} \quad \mathrm{NAD}^{+}+\mathrm{H}^{+}
$$

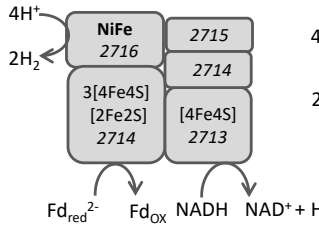

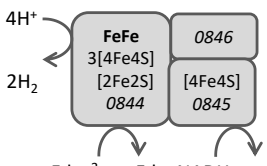

$\mathrm{Fd}_{\mathrm{red}}{ }^{2-} \mathrm{Fd}_{\mathrm{OX}} \mathrm{NADH} \mathrm{NAD}^{+}+\mathrm{H}^{+}$
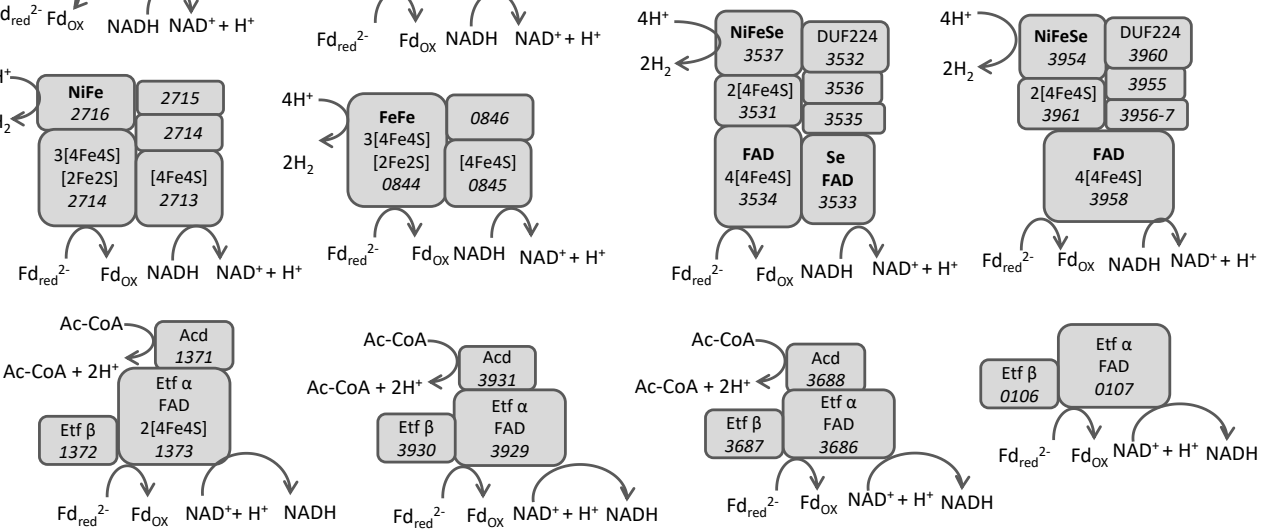

$\mathrm{Fd}_{\mathrm{red}}{ }^{2-} \quad \mathrm{Fd}_{\mathrm{Ox}} \mathrm{NAD}^{+}+\mathrm{H}^{+} \mathrm{NADH}$

$\mathrm{Fd}_{\text {red }}{ }^{2-} \mathrm{Fd}_{\mathrm{Ox}} \mathrm{NAD}^{+}+\mathrm{H}^{+} \mathrm{NADH}$ 


\section{B. Syntrophomonas wolfei}

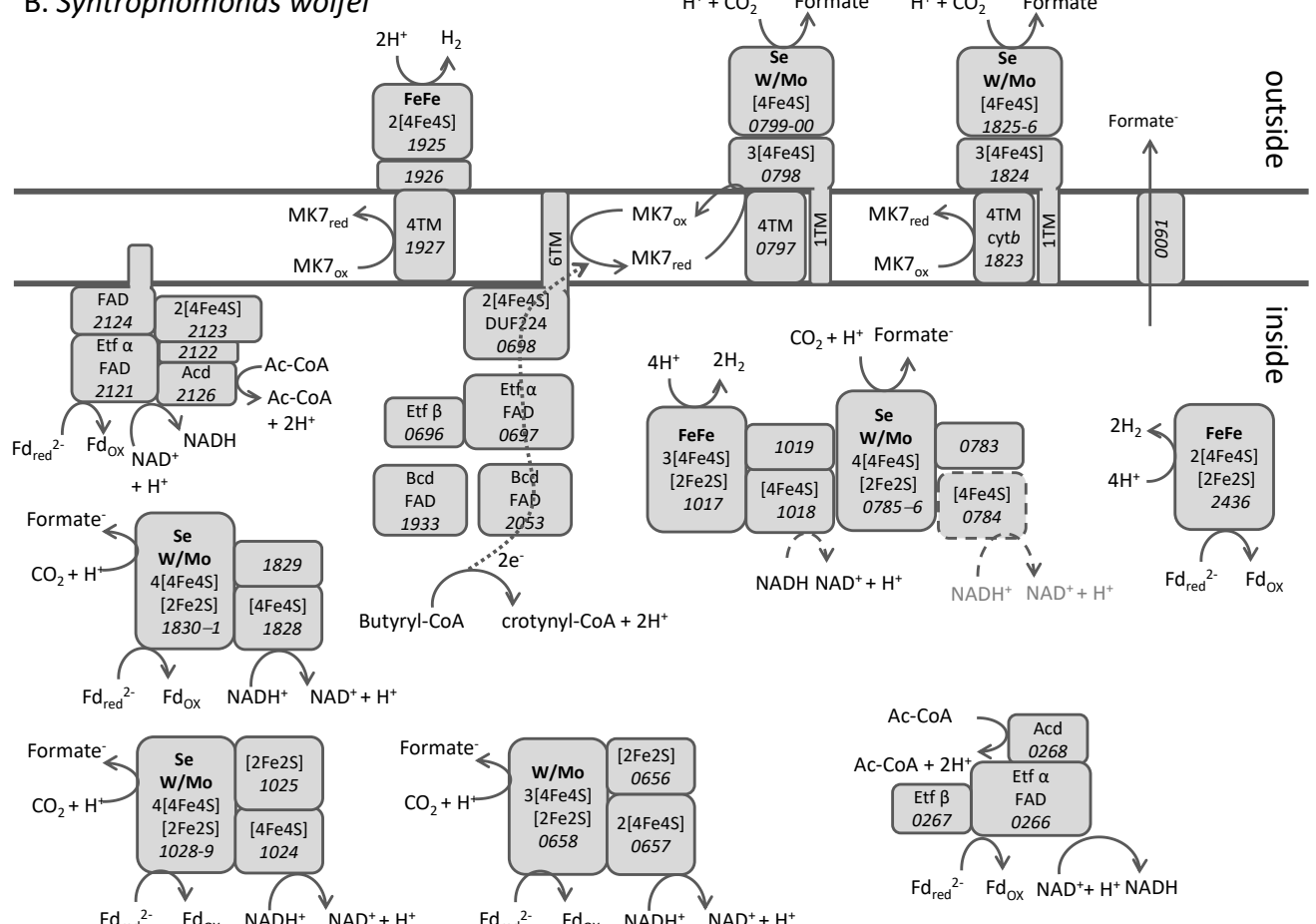

\section{Syntrophothermus lipocalidus}
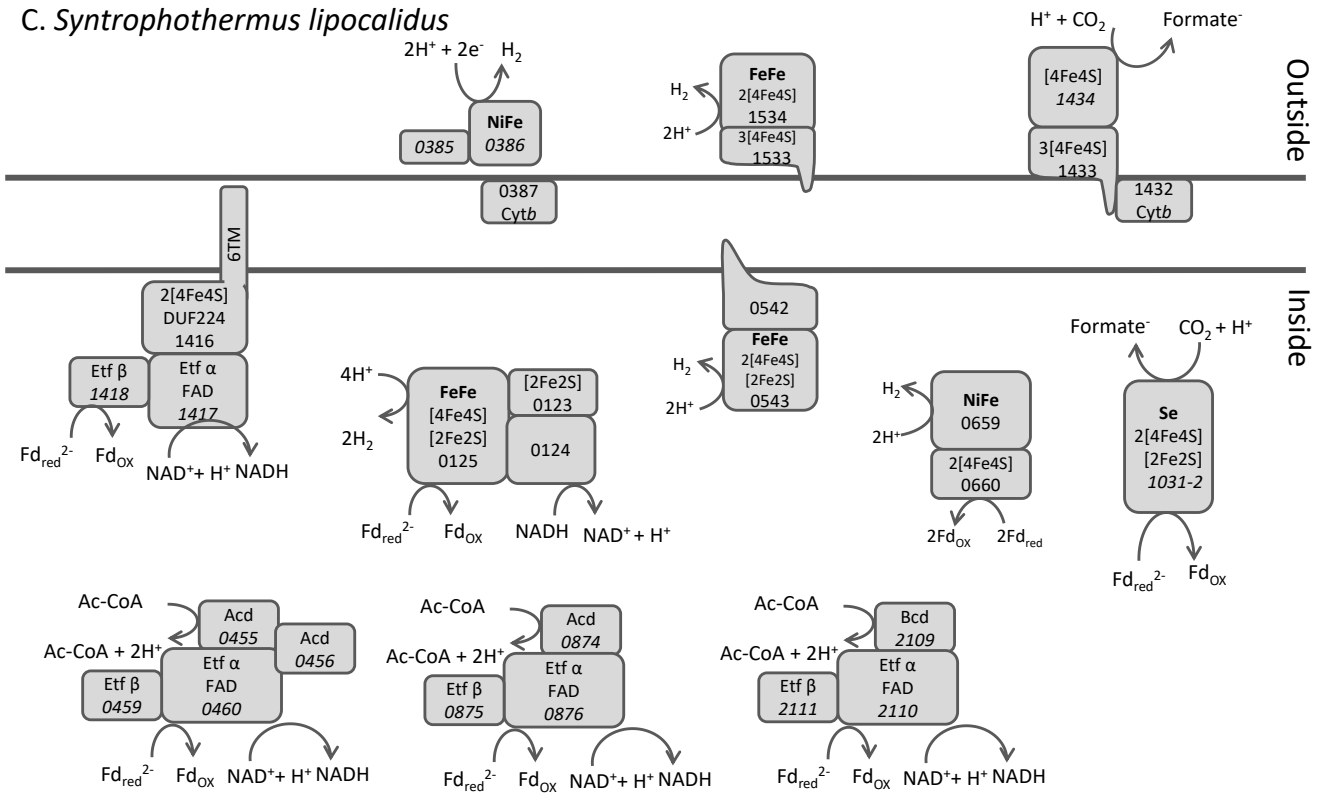


\section{Syntrophus aciditrophicus}
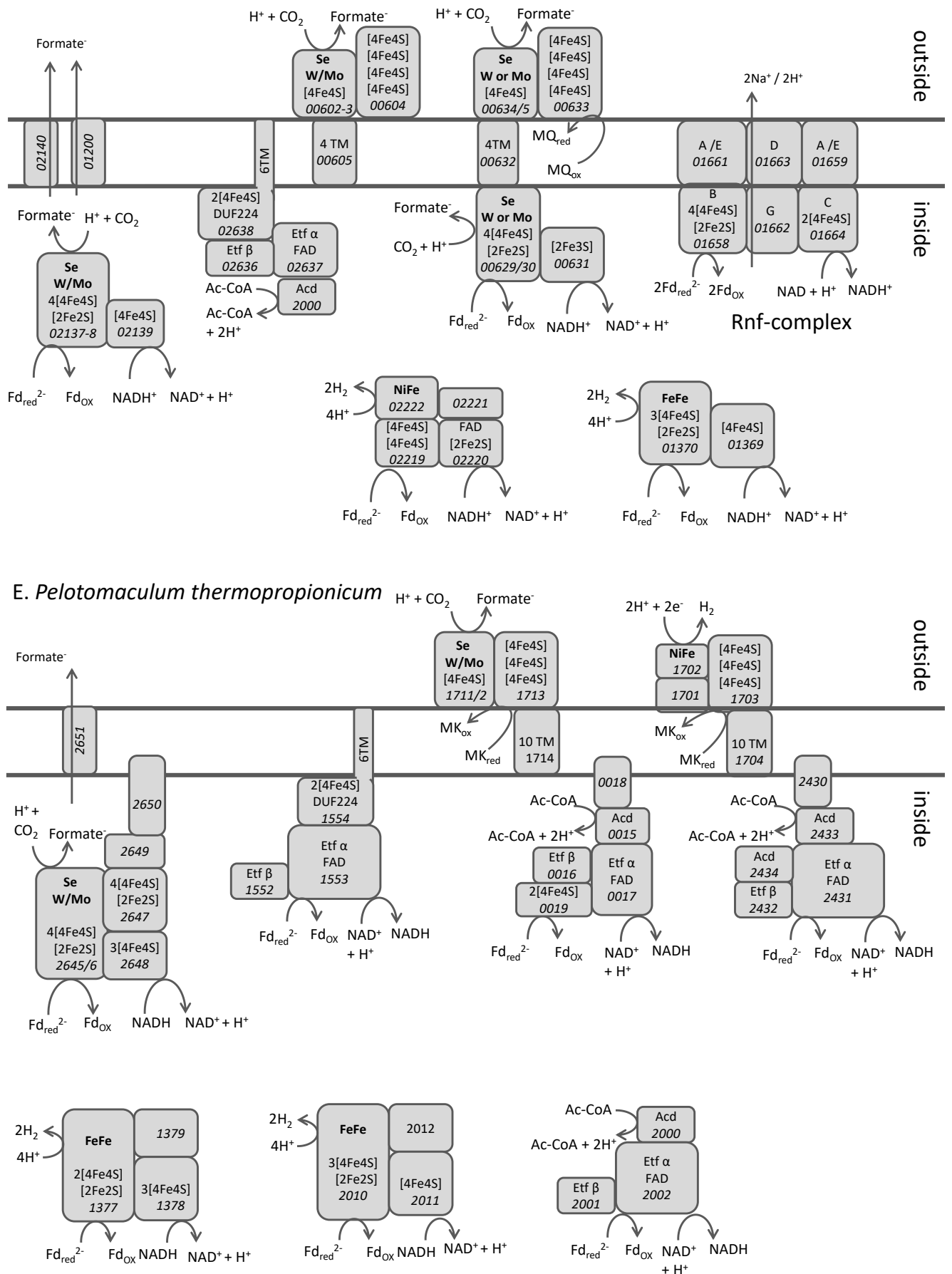

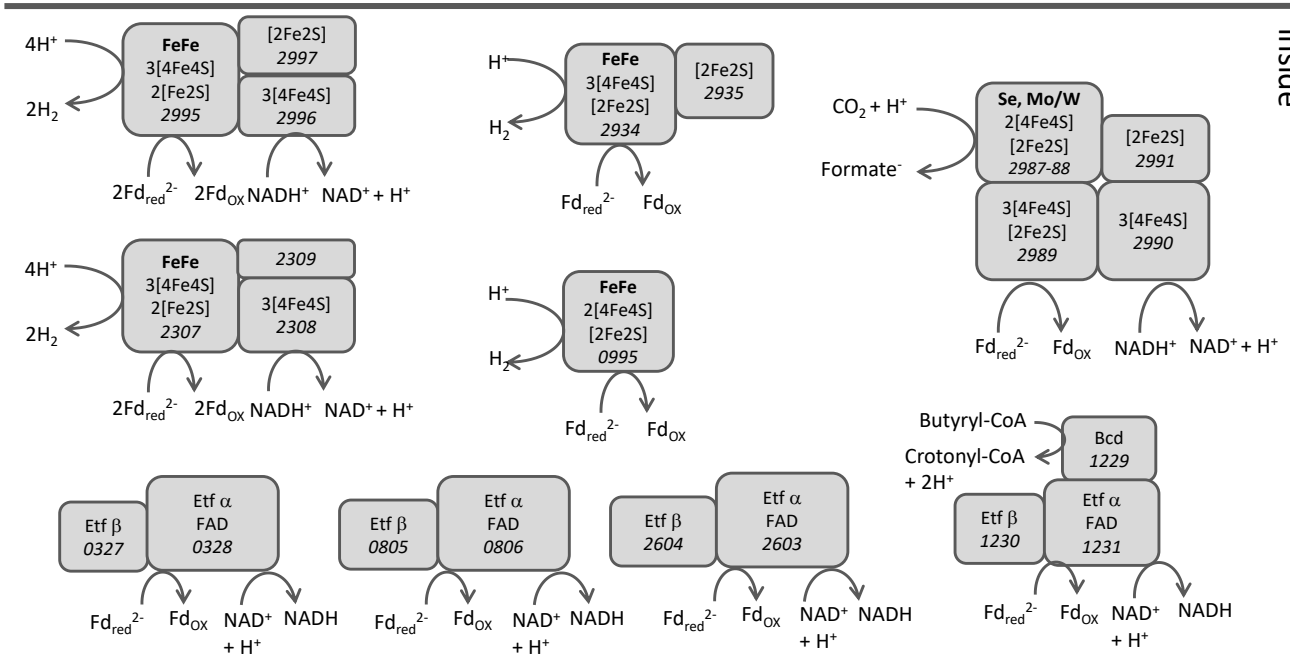

\section{G. Desulfobulbus propionicus}

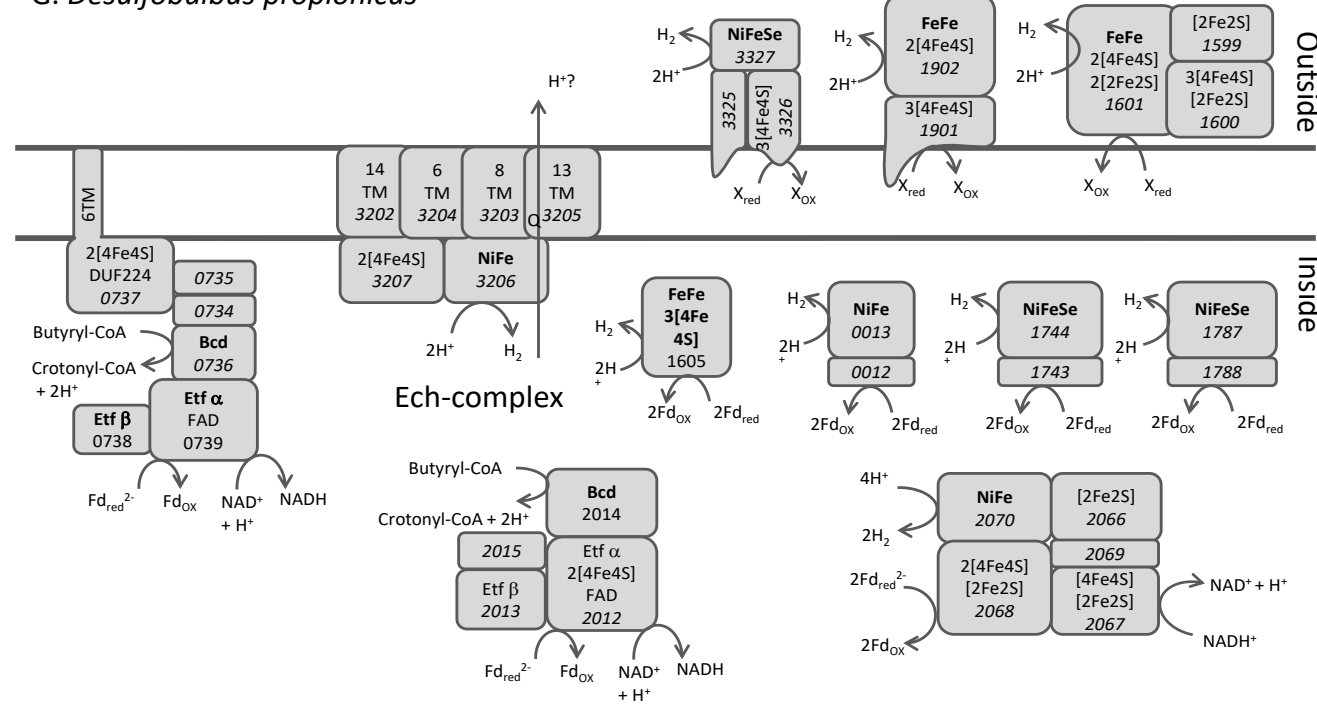

Fig. S1: Energy converting enzyme complexes predicted from the genomes of bacteria that can degrade propionate and butyrate in syntrophic growth with methanogens; Syntrophobacter fumaroxidans (A), Syntrophomonas wolfei (B), Syntrophothermus lipocalidus (C), Syntrophus aciditrophicus (D), Pelotomaculum thermopropionicum (E), and from those that cannot grow in syntrophic growth with methanogens; Desulfotomaculum kuznetsovii (F), and Desulfobulbus propionicus (G) 
Syntrophy as the microbiological example of Hegel's slave-master dialectics 
$m$

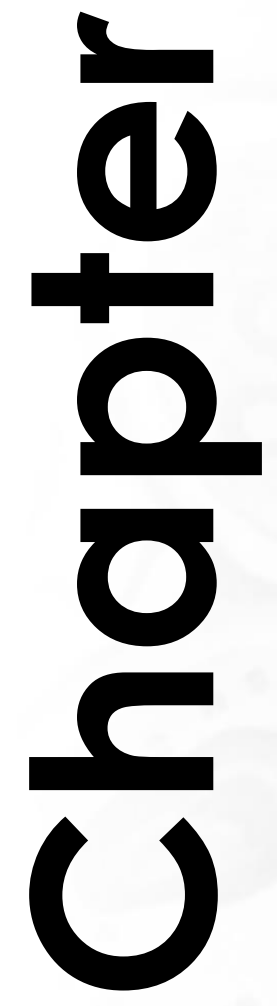




\section{CHAPTER 3}

\section{Metabolic flexibility of Syntrophobacter fumaroxidans: Syntrophic vs sulfate- reducing lifestyle with propionate as growth substrate}

Vicente T. Sedano-Núñez, Alfons J. M. Stams and Caroline M. Plugge 


\begin{abstract}
Syntrophobacter fumaroxidans is a sulfate-reducing bacterium capable of oxidizing propionate in pure culture coupled to sulfate reduction and in syntrophy with methanogens in the absence of sulfate. The role of $S$. fumaroxidans as a syntroph has been studied, but its metabolic flexibility and adaptation to changing environmental conditions has never been assessed. We hypothesized that the syntrophic metabolism of $S$. fumaroxidans is more efficient than propionate oxidation coupled to sulfate reduction. Perturbations in sulfidogenic pure cultures of S. fumaroxidans and in methanogenic cocultures of the bacteria with Methanospirillum hungatei were performed. The addition of sulfate to syntrophic cocultures triggered a metabolic shift in $S$. fumaroxidans. Sulfate started to be reduced and the methane production decreased up to $40 \%$. The addition of $M$. hungatei to the sulfidogenic axenic cultures of $S$. fumaroxidans did not lead to an adaptation of $S$. fumaroxidans to a syntrophic lifestyle. Complementary trials showed inhibition of the methanogenic partner at sulfide concentrations present at the moment of the perturbation (above $10 \mathrm{mM}$ ). This hampered the metabolic shift of $S$. fumaroxidans towards syntrophy. Desulfovibrio desulfuricans G11 was then used as an alternative syntrophic partner that could scavenge $\mathrm{H}_{2}$ and/or formate from $S$. fumaroxidans while tolerating high levels of sulfide in the medium. Growth of $D$. desulfuricans in the coculture with $S$. fumaroxidans was verified with qPCR. Although growth of $D$. desulfuricans in the coculture was shown, it could not be clearly shown that $S$. fumaroxidans switched its metabolism from sulfidogenesis to syntrophy.
\end{abstract}

Keywords: Syntrophy, sulfate-reducing bacteria, propionate oxidation, metabolic flexibility, methanogens, sulfide inhibition. 


\section{Introduction}

In anaerobic environments where the amount of inorganic electron acceptors such as nitrate, sulfate, sulfur or oxidized metal ions is low, syntrophic associations between acetogenic bacteria and methanogenic archaea become essential for the complete degradation of organic compounds to methane and $\mathrm{CO}_{2}$ (Schink and Stams, 2013). In such conditions, degradation of propionate, butyrate and long chain fatty acids is only possible if the products acetate, hydrogen and formate are kept in low concentrations by methanogens (Schink, 1997; Sieber et al., 2012). If the situation changes and sulfate becomes available, sulfate-reducing bacteria (SRB) compete with the methanogens for hydrogen, formate and acetate, but also with entire syntrophic methanogenic communities for substrates like propionate and butyrate (Muyzer and Stams, 2008). While many SRB can grow without sulfate and are engaged in syntrophic associations with methanogens, others lack this ability (Worm et al., 2014). Moreover, some members of generally recognized sulfate-reducing taxonomic groups seem to have lost their ability to respire anaerobically with sulfate (de Bok et al., 2005; Imachi et al., 2006; Plugge et al., 2011).

The metabolic flexibility of SRB to form syntrophic associations despite their ability to reduce sulfate and oxidize fatty acids on their own, enhances their chances of survival when changes in the environment occur and affects the spatial distribution of microbial genera (Carbonero et al., 2014). In general terms, sulfate reduction is favoured over methanogenesis when sufficient sulfate is present (Lovley and Klug, 1983; Muyzer and Stams, 2008). Nevertheless, the growth rates of some SRB indicate a more efficient metabolism when degrading propionate in syntrophy than by reducing sulfate (van Kuijk and Stams, 1995; Wallrabenstein et al., 1995a; Wallrabenstein et al., 1995b; Harmsen et al., 1998). It is important to assess the metabolic flexibility of SRB in fluctuating environments in order to gain knowledge about the dynamics and resilience of microbial communities.

Syntrophobacter fumaroxidans is a propionate-degrading bacterium able to couple propionate oxidation to sulfate reduction. Degradation of propionate coupled to fumarate reduction is also possible (Harmsen et al., 1998). In the absence of inorganic electron acceptors, $S$. fumaroxidans requires an efficient removal of $\mathrm{H}_{2}$ - and formate from the environment, which is usually achieved by growing in syntrophy with $\mathrm{H}_{2}$ or formate-consuming microorganisms. Although these syntrophic associations are generally described with methanogenic archaea, other $\mathrm{H}_{2}$ or formate scavengers can function as syntrophic partners (Dong et al., 1994). Sulfidogenic growth of $S$. fumaroxidans has been less studied than its ability to grow syntrophically. Yet it is known that the growth rate of this bacterium with propionate coupled to sulfate reduction is much slower than when grown in syntrophy with methanogenic archaea (van Kuijk and Stams, 1995; Scholten and Conrad, 2000). 
Desulfovibrio desulfuricans G11 is a microorganism able to couple the oxidation of lactate, ethanol, formate or $\mathrm{H}_{2}$ to the reduction of sulfate to sulfide or of nitrate to ammonium (Sheik et al., 2017). In the absence of these electron acceptors, $D$. desulfuricans ferments lactate and alcohols and produces acetate, formate, $\mathrm{H}_{2}$ and $\mathrm{CO}_{2}$. Moreover, syntrophic growth on formate with a hydrogenotrophic methanogen has also been reported (Dolfing et al., 2008). However, D. desulfuricans is not able to catabolize butyrate, propionate or acetate.

The role of $S$. fumaroxidans as a syntroph has been widely studied and its importance in methanogenic environments documented (McMahon et al., 2001; McMahon et al., 2004; Stams et al., 2012). However, the metabolic flexibility to changes in the environment and the preferred lifestyle of this bacterium requires more investigation. By combining the capacities of $D$. desulfuricans as scavenger of $\mathrm{H}_{2}$ and formate, and the alternative lifestyles of $S$. fumaroxidans to grow as a sulfate reducer or as a syntroph with methanogenic archaea, we studied the metabolic adaptability of $S$. fumaroxidans to changing environments provoked by different perturbations.

\section{Materials and methods}

\section{Organisms and growth conditions}

Syntrophobacter fumaroxidans MPOB $^{\mathrm{T}}$ (DSM 10017) was cultivated under anoxic conditions in basal medium as described previously (Stams et al., 1993). Axenic sulfidogenic cultures of $S$. fumaroxidans were grown with $20 \mathrm{mM}$ sodium propionate and $20 \mathrm{mM}$ sodium sulfate. Syntrophic cocultures of $S$. fumaroxidans with the methanogenic archaeon Methanospirillum hungatei strain JF1'T (DSM 864) were grown on $30 \mathrm{mM}$ of sodium propionate. Axenic cultures of $M$. hungatei were grown with formate $(40 \mathrm{mM})$ or with hydrogen in the headspace (1.7 atm $\mathrm{H}_{2} / \mathrm{CO}_{2}$ 80:20 $\mathrm{vol} / \mathrm{vol}$ ) and supplemented with $1 \mathrm{mM}$ sodium acetate. Desulfovibrio desulfuricans G11 (DSM 7057) was cultured with $40 \mathrm{mM}$ of formate or hydrogen (1.7 atm $\mathrm{H}_{2} / \mathrm{CO}_{2}$ $80: 20 \mathrm{vol} / \mathrm{vol}$ ) as electron donor, and $20 \mathrm{mM}$ sodium sulfate as electron acceptor. Organisms were batch cultured in duplicate or triplicate at $37{ }^{\circ} \mathrm{C}$ in $250 \mathrm{ml}$ flasks with $110 \mathrm{ml}$ medium for methanogenic cocultures and 1-liter flasks with $550 \mathrm{ml}$ medium for sulfidogenic cultures. Anaerobic conditions were provided by an 80:20 ( $\mathrm{vol} / \mathrm{vol}$ ) gas mixture headspace of $\mathrm{N}_{2} / \mathrm{CO}_{2}$, or $\mathrm{H}_{2} / \mathrm{CO}_{2}$ when hydrogen was the electron donor (Plugge, 2005).

For the starvation tests, active cultures of $M$. hungatei growing on $\mathrm{H}_{2} / \mathrm{CO}_{2}$ or formate were used to inoculate bottles with anaerobic basal medium complemented with vitamins, $\mathrm{N}_{2} / \mathrm{CO}_{2}(80: 20 \mathrm{vol} / \mathrm{vol})$ in the gas phase and reducing solution, but without adding any electron donor or carbon source. These bottles were incubated at $37^{\circ} \mathrm{C}$ and after various periods of starvation the electron donor was added by changing the gas phase to $\mathrm{H}_{2} / \mathrm{CO}_{2}(80: 20 \mathrm{vol} / \mathrm{vol})$ or by adding formate $(30 \mathrm{mM})$ to the bottles. 
Substrate consumption and product formation were monitored. A similar approach was followed for the starvation test on Desulfovibrio desulfuricans, albeit sodium sulfate was added to the basal medium as electron acceptor.

\section{Growth monitoring}

Growth was monitored by measuring the optical density at $600 \mathrm{~nm}$ (OD600), as well as measuring propionate, sulfate, $\mathrm{H}_{2}$ or formate consumption and product formation (methane, acetate, and sulfide). Organic acids were measured with a Thermo Scientific Spectrasystem high-performance liquid chromatograph (HPLC) equipped with a Varian Metacarb $67 \mathrm{H} 300 \mathrm{~mm}$ column kept at $45^{\circ} \mathrm{C}$ and run with $0.005 \mathrm{mM}$ sulfuric acid as eluent at a flow rate of $0.6 \mathrm{ml} \mathrm{min}{ }^{-1}$. Sulfate concentrations were quantified using a Dionex ICS-1000 ion chromatograph (Dionex) equipped with an IonPac AS22 column and $4.5 \mathrm{mM}$ carbonate/1.4 mM bicarbonate eluent at a flow rate of $1.2 \mathrm{ml} \mathrm{min}^{-1}$. Hydrogen and methane were determined with a CompactGC gas chromatograph (Global Analyser Solutions) with a Molsieve 5A PLOT of $0.53 \mathrm{~mm}$. Hydrogen sulfide was measured by a colorimetric method (Cline, 1969).

\section{Perturbation events}

Various perturbation agents were applied to two different growth conditions of $S$. fumaroxidans. For the methanogenic cocultures of $S$. fumaroxidans and $M$. hungatei the perturbations were: the addition of sulfate to the medium (M+Sulf); the addition of sulfate and 20\% inoculum of Desulfovibrio desulfuricans G11 (M+SulfG11); the addition of sulfate plus the inactivation of the methanogenic partner by adding $1 \mathrm{mM}$ sodium 2-bromoethanesulfonate (BES) (M+SulfBES) and the supply of sulfate and $20 \%$ inoculum of active $D$. desulfuricans G11 coupled with the inactivation of the methanogen with BES (M+SulfBESG11). The sulfidogenic axenic cultures of $S$. fumaroxidans were perturbed in the following way: by adding $45 \%$ inoculum of active M. hungatei in the sulfidogenic culture (S+JF1) or by adding $45 \%$ inoculum of active D. desulfuricans $\mathrm{G} 11$ in the sulfidogenic culture (S+G11). When M. hungatei or D. desulfuricans G11 were used as perturbation agents, equal amounts of cells grown with $\mathrm{H}_{2} / \mathrm{CO}_{2}$ or formate were inoculated.

\section{Quantitative PCR}

A quantitative PCR (qPCR) analysis was performed in the treatments where $D$. desulfuricans G11 was introduced as part of the perturbations. Two sampling points were established to harvest cells and extract DNA from the biological replicates. The first sampling point took place right after the perturbation event and the second at the end of the experiment. DNA was extracted using the FastDNA® Spin Kit for Soil (MP Biomedicals, Santa Ana, CA) according to the manufacturer's protocol with two 45-second beat beating steps using a FastPrep Instrument (MP Biomedicals). DNA 
concentrations were quantified with Qubit® 2.0 Fluorometer (Invitrogen, Carlsbad, CA). Amplifications were done in triplicates in a CFX384 Real-Time PCR Detection System (Bio-Rad Laboratories, Inc., Hercules, CA). The reaction mixture consisted of 5 pl iTaq Universal SYBR Green Supermix (Bio-Rad Laboratories, Inc., Hercules, $\mathrm{CA}), 0.2 \mu \mathrm{l}$ reverse primer $[50 \mu \mathrm{M}], 0.2 \mu \mathrm{l}$ forward primer $[50 \mu \mathrm{M}]$, and $2 \mu \mathrm{l}$ of template DNA [10 ng/ $\mu \mathrm{l}]$. PCR grade water was used to fill up the reaction mixture to $10 \mathrm{\mu l}$. Specific $16 \mathrm{~S}$ rRNA primers designed for $D$. desulfuricans were used DSVspG11 201f (5'-ACCTCTGCTTGCATGTTACC-3') and DSVspG11 $471 r$ (5'CTGATTAGCACAGTGCGGTTT-3'). The qPCR amplification proceeded as follows: a pre-denaturing step $95^{\circ} \mathrm{C}(5 \mathrm{~min}), 40$ cycles of $95^{\circ} \mathrm{C}(30 \mathrm{~s}), 62^{\circ} \mathrm{C}(40 \mathrm{~s}), 72^{\circ} \mathrm{C}(40 \mathrm{~s})$, $80^{\circ} \mathrm{C}(25 \mathrm{~s})$ as optimized by (Junicke et al., 2014). PCR products were checked for specificity by a melting curve analysis $\left(72-95^{\circ} \mathrm{C}\right)$ after each amplification step. Triplicate standard curves were obtained with 10 -fold serial dilutions ranged from $1 \times 10^{8}$ to $1 \times 10^{1}$ copies per $\mu$ l of $16 \mathrm{~S}$ rDNA of $D$. desulfuricans G11. The efficiency of the reactions was $100 \%$ and the $\mathrm{R}^{2}$ of the standard curves 0.996 . Quantification of specific bacteria was expressed as increase in $16 \mathrm{~S}$ rRNA gene copies.

\section{Results and discussion}

The results of all perturbations applied to methanogenic cocultures and sulfidogenic cultures of $S$. fumaroxidans are summarized in Table 3.1. Syntrophic methanogenic cocultures reduced sulfate when this compound was added to the growth medium. However, the adaptation of $S$. fumaroxidans in the methanogenic coculture to a sulfidogenic lifestyle varied along with other factors involved in the different perturbations (Figure 3.1). In our analysis, we have compartmentalized this adaptation in two stages, week one and week two after the perturbations took place. Thus, Table 3.1 shows substrate degradation and product formation, measured one and two weeks after the perturbations events.

For the first week after the perturbations, the highest propionate degradation was observed in treatment M+Sulf, where only sulfate was the perturbation agent added to the medium. Although the methanogenic syntrophic lifestyle of $S$. fumaroxidans in this treatment was still important as the levels of methane produced indicate, the amounts of sulfate reduced and sulfide formed seem to indicate that the bacteria channelled more of its reducing equivalents to the reduction sulfate. However, this is not the case as it will be discussed below.

A similar sulfate reduction as in M+Sulf can be noticed in treatment M+SulfBES, where the methanogenic partner was inhibited with BES and no methane was formed. Taking treatment M+SulfBES as a reference, we can extrapolate and infer that an approximate amount of $\sim 9.2 \mathrm{mM}$ of propionate was oxidized in treatment $\mathrm{M}+$ Sulf coupled to sulfate reduction, as this was the amount of propionate oxidized in treatment M+SulfBES, where propionate oxidation coupled to methane formation 
was inhibited. During propionate degradation reducing equivalents are formed. The reduced forms of electron carriers, such as NADH or reduced ferredoxin need to be re-oxidized to keep propionate degradation going on. S. fumaroxidans can couple the re-oxidation of these carriers to proton or $\mathrm{CO}_{2}$ reduction and form $\mathrm{H}_{2}$ or formate, respectively, that need to be scavenged by a syntrophic partner; or it can also reoxidize these equivalents on its own by reducing sulfate and produce sulfide. Theoretically the oxidation of $21 \mathrm{mM}$ of propionate in $\mathrm{M}+$ Sulf generates 63 reducing equivalents, which can be used in the formation of $15.8 \mathrm{mM}$ of sulfide and/or methane. Since approximately $7 \mathrm{mM}$ of sulfate was reduced in M+Sulf, the methane formation should be of $8.8 \mathrm{mM}$, which requires $11.7 \mathrm{mM}$ of propionate. Thus, of the $21 \mathrm{mM}$ of propionate degraded in M+Sulf, approximately $9.2 \mathrm{mM}$ was coupled to sulfate reduction and $11.7 \mathrm{mM}$ to the production of hydrogen and/or formate. Therefore, syntrophy was still the predominant lifestyle of S. fumaroxidans despite the availability of sulfate in the medium.

The amount of propionate degraded in treatment M+Sulf shows a highly active $S$. fumaroxidans that efficiently made use of an additional inorganic electron acceptor added to the medium, in this case sulfate. However, it also kept transferring hydrogen and/or formate to the methanogenic partner.

The addition of $D$. desulfuricans as part of the perturbations in treatments M+SulfG11 and M+SulfBESG11 hindered sulfate reduction during the first week, as less sulfate was reduced in comparison to those treatments where G11 was not added. This hampering effect of G11 affects sulfate reduction, but not propionate oxidation. In treatment M+SulfG11, where the methanogen was not inhibited, a high propionate oxidation is observed, most probably by the syntrophic association of $S$. fumaroxidans with $M$. hungatei as the methane produced also indicates. On the other hand, it was not certain at this stage to deduce if sulfate in treatments M+SulfG11 and M+SulfBESG11 was reduced by $D$. desulfuricans or by $S$. fumaroxidans.

For the second week, the substrate consumption and methane production rates diverged from week one as a result of the adaptation of $S$. fumaroxidans to the new environmental conditions. The amount of propionate degraded was less in most of the treatments, with the exception of treatments with M+SulfBESG11. In some cases, the lower amount of propionate oxidized is due to the exhaustion of this substrate during the second week. For instance, in the control treatment and in $\mathrm{M}+$ Sulf most of the propionate available was degraded during the first week. Nonetheless, the drop in the methane formation in treatments M+Sulf and $\mathrm{M}+\mathrm{SulfG} 11$ is remarkable. 


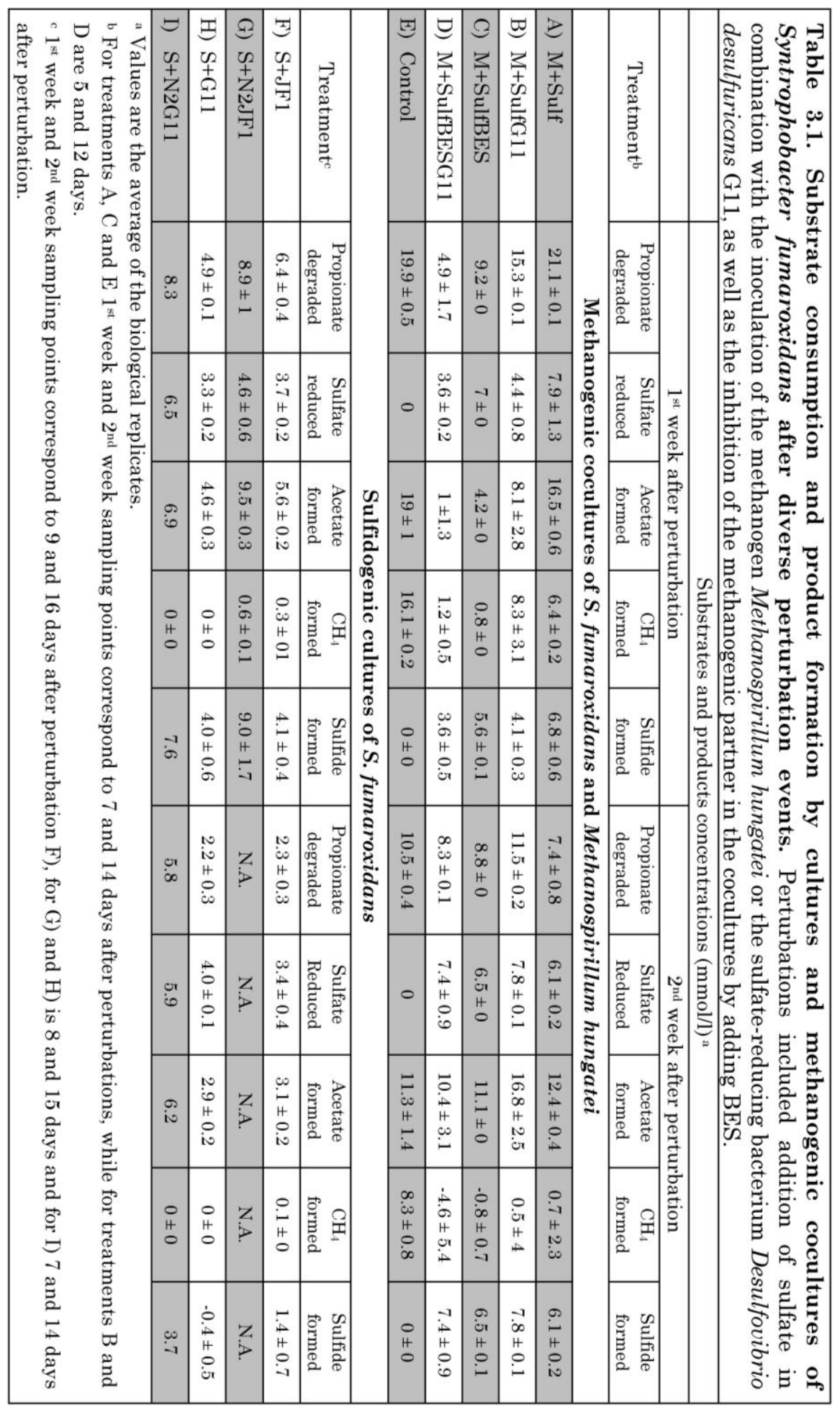


In these treatments, the methanogens might have been inhibited by the high levels of sulfide accumulated in the media after the first week of the perturbations (Figure 3.1). Thus, the syntrophic interaction of $M$. hungatei with $S$. fumaroxidans was hampered when the archaeon was not able to scavenge the $\mathrm{H}_{2}$ and formate produced by the bacterium during propionate oxidation. This toxic effect of sulfide on the methanogen and the combinations with the inhibitory effect of treatments where BES was added allowed us to make some interesting speculations.
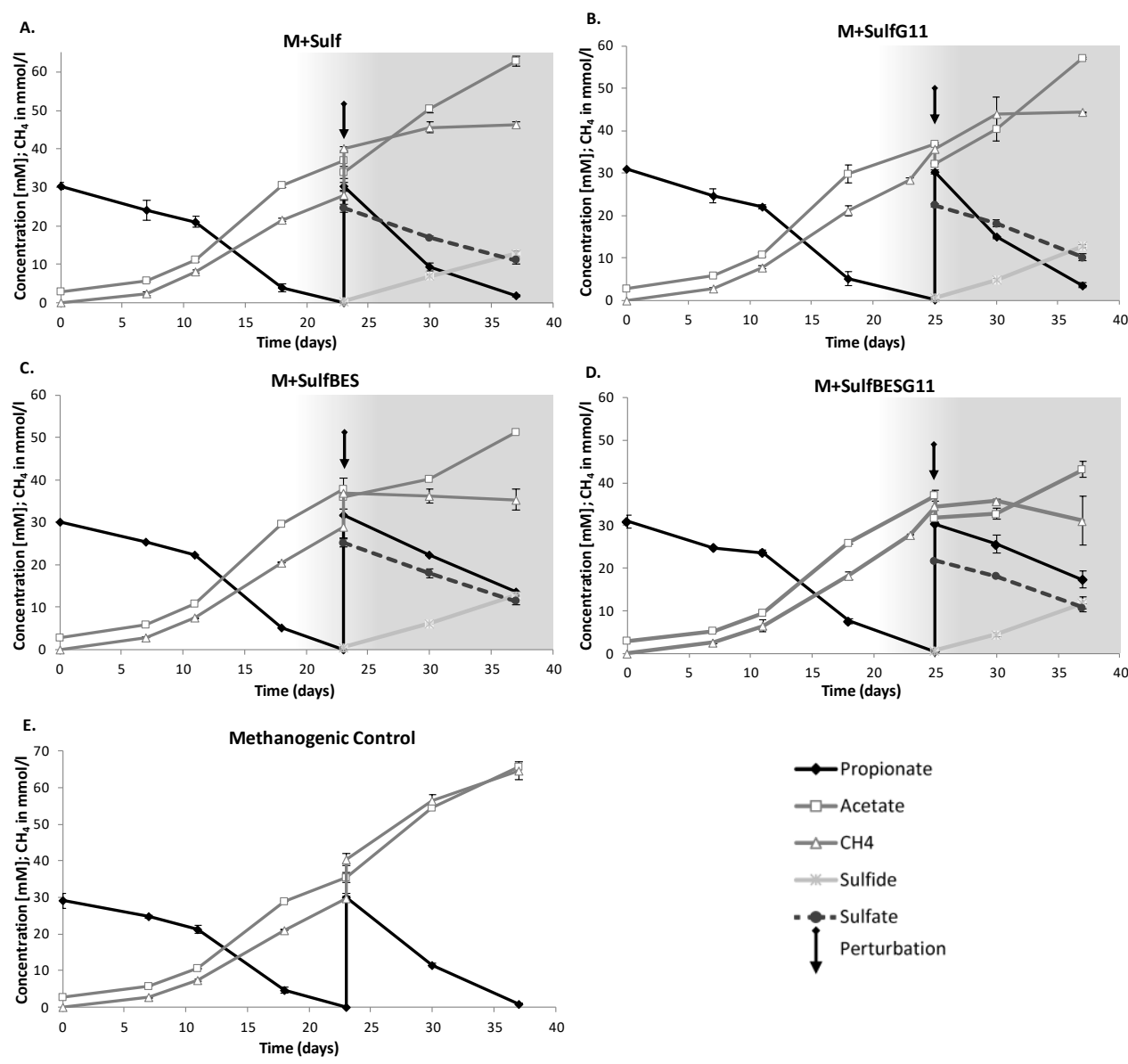

Figure 3.1. Methanogenic cocultures of $S$. fumaroxidans with M. hungatei exposed to different perturbation events. (A) Addition of sodium sulfate to the medium. (B) Addition of sulfate and inoculation of active D. desulfuricans G11. (C) Inhibition of the methanogenic partner by the addition of 2-bromoethanesulfonate (BES) (D) Inhibition of the methanogenic partner with BES and inoculation of active $D$. desulfuricans G11. (E) Control with a methanogenic coculture of S. fumaroxidans with M. hungatei. 
During the first week M+Sulf and M+SulfBES reduced $\sim 7 \mathrm{mM}$ of sulfate. For the second week, all the treatments, except the control, in average reduced $\sim 7 \mathrm{mM}$ of sulfate and the differences in these reductions and in the amounts of propionate oxidized were as follows: M+Sulf, reduced less sulfate of all because it exhausted all the propionate. M+SulfBES had enough propionate but the methanogen was inhibited, and therefore only $S$. fumaroxidans performed sulfidogenesis and consumed similar amounts of propionate and sulfate as in week one. In M+SulfBESG11 more sulfate was reduced than in M+SulfBES, but less propionate oxidation was noticed. These values do not fit the electron recovery calculations where $8.3 \mathrm{mM}$ of propionate would result in a maximum reduction of $6.2 \mathrm{mM}$ of sulfate. The presence of $D$. desulfuricans G11 might have an effect in the differences on sulfate reduction in contrast to when G11 is not present in the medium.

Finally, the highest amount of sulfate reduced and propionate degraded during week two were observed in treatment M+SulfG11. Although here we only observe the formation of traces of methane, we speculate that despite the inhibition of the methanogen by the high concentrations of sulfide in the medium, methane production endures as much as it is possible by the methanogen, while Syntrophobacter still couples propionate oxidation to the production of hydrogen and/or formate. Thus, although the methanogen cannot retrieve the hydrogen and formate produced by Syntrophobacter, D. desulfuricans G11 might take that role and so we observed a higher propionate oxidation and sulfate reduction than when $D$. desulfuricans G11 was not present.

The values presented in Table 3.1 do not always perfectly fit the stoichiometry. In some cases, it is possible that the accumulated methane leaked from the overpressurized bottles. This is supported by the negative values of methane in treatments M+SulfBES and M+SulfBESG11 in week 2. A table with electron and carbon balance is presented as Supplementary Material Table S3.1.

The perturbation in the sulfidogenic cultures of $S$. fumaroxidans took place at the mid-exponential phase (Figure 3.2). At this point, sulfide levels were $\sim 10 \mathrm{mM}$. Although toxicity of hydrogen sulfide is $\mathrm{pH}$ dependent and the toxic form $\left(\mathrm{H}_{2} \mathrm{~S}\right)$ is dominant at acidic $\mathrm{pH}$ levels $(<6)$, inhibitory effects on anaerobic microorganisms, particularly non-sulfate reducers, are reported already above $3 \mathrm{mM}$ at $\mathrm{pH} 7$ (Lens et al., 1998; Paulo et al., 2015).

The absence of methane production in treatment S+JF1 after the addition of $M$. hungatei indicates that the expected metabolic shift of $S$. fumaroxidans towards a syntrophic lifestyle did not occur (Figure 3.2.A). The syntrophic interaction between the bacterium and the methanogen depends on the equilibrium maintained at the pool of metabolites exchanged among these microorganisms. It is not clear whether $S$. fumaroxidans did not provide the hydrogen and formate required by M. hungatei, 
or the methanogen did not consume such compounds. Considering the high sulfide concentrations in the medium, it is probable that the methanogen was inhibited. To corroborate the inhibitory effect of hydrogen sulfide on $M$. hungatei, pure cultures of the methanogen growing in $\mathrm{H}_{2} / \mathrm{CO}_{2}$ or formate were tested at different sulfide concentrations (Figure 3.3).
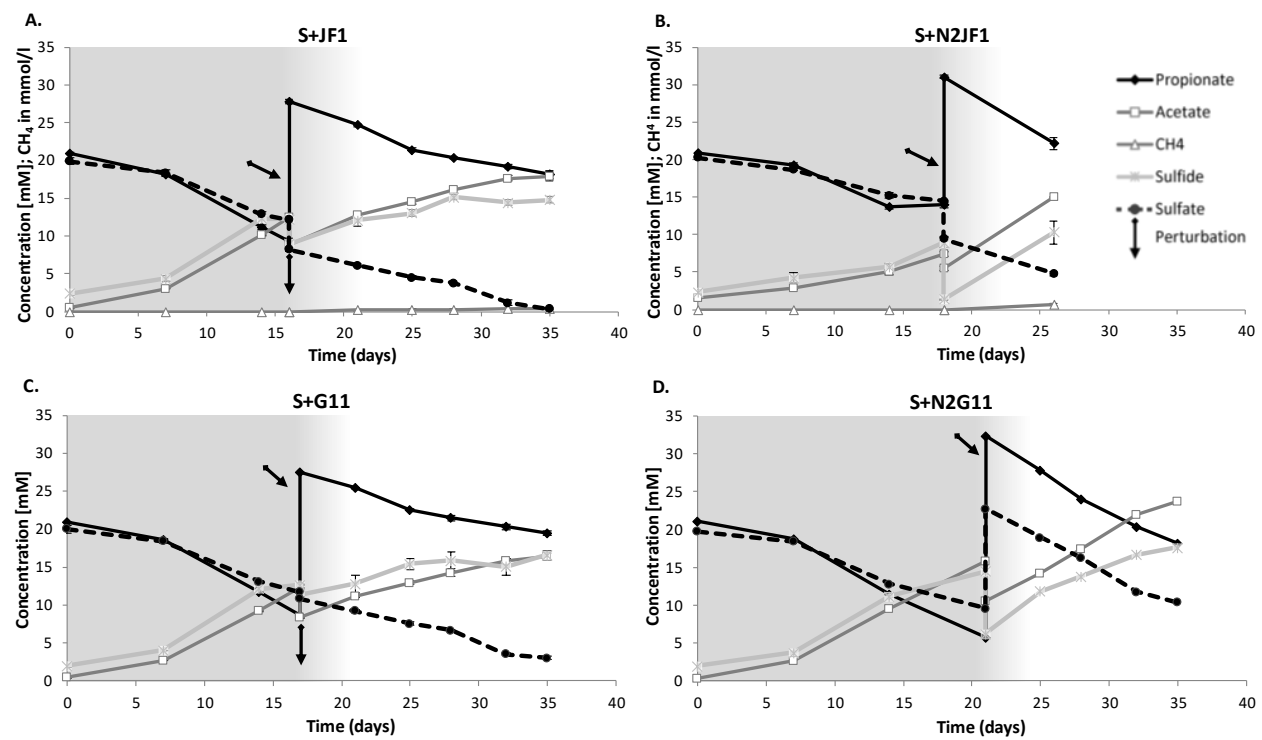

Figure 3.2. Response of sulfate-reducing cultures of $S$. fumaroxidans to the addition of hydrogen- and formate-scavenging microorganisms. (A) Inoculation of active $M$. hungatei at day 16. (B) Inoculation of active $M$. hungatei at day 18 after sulfide was flushed out with $\mathrm{N}_{2}$. (C) Inoculation of active D. desulfuricans $\mathrm{G} 11$ at day 17 (D) Inoculation of active $D$. desulfuricans after sulfide was flushed out with $\mathrm{N}_{2}$ at day 21 .

Levels above $4 \mathrm{mM}$ of sulfide had a significant impact on the methane formation and substrate consumption in cultures of $M$. hungatei growing with $\mathrm{H}_{2} / \mathrm{CO}_{2}$. Cultures growing on formate showed a slightly higher tolerance to sulfide $(5 \mathrm{mM})$, although not high enough to strive at the levels $(10 \mathrm{mM})$ present in the sulfidogenic environment of treatment S+JF1. We tried to decrease the sulfide levels of the sulfidogenic cultures prior the inoculation of the methanogen, by flushing the media in the bottles with $\mathrm{N}_{2}$ and exchanging the gas phase with $\mathrm{N}_{2} / \mathrm{CO}_{2}$ on treatment S+N2JF1. Although the sulfide concentration dropped to $1.3 \mathrm{mM}$ and the $\mathrm{pH}$ only rose to 7.2 , the flushing approach proved ineffective and no methane was detected after the perturbation (Figure 3.2.B).

In addition to the sulfide inhibitory effect on the methanogen, the possibility of a delayed metabolic shift of the bacterium was also considered. The growth rate of $S$. fumaroxidans growing on propionate coupled to sulfate reduction is very low (0.024 day $^{-1}$ ) (van Kuijk and Stams, 1995) in comparison with syntrophic growth with 
propionate $\left(0.17\right.$ day $\left.^{-1}\right)$ (Harmsen et al., 1998). If the adaptation time required by $S$. fumaroxidans to switch its metabolism was longer than the survival time of the methanogen without an available electron donor, the syntrophic association would not take place.
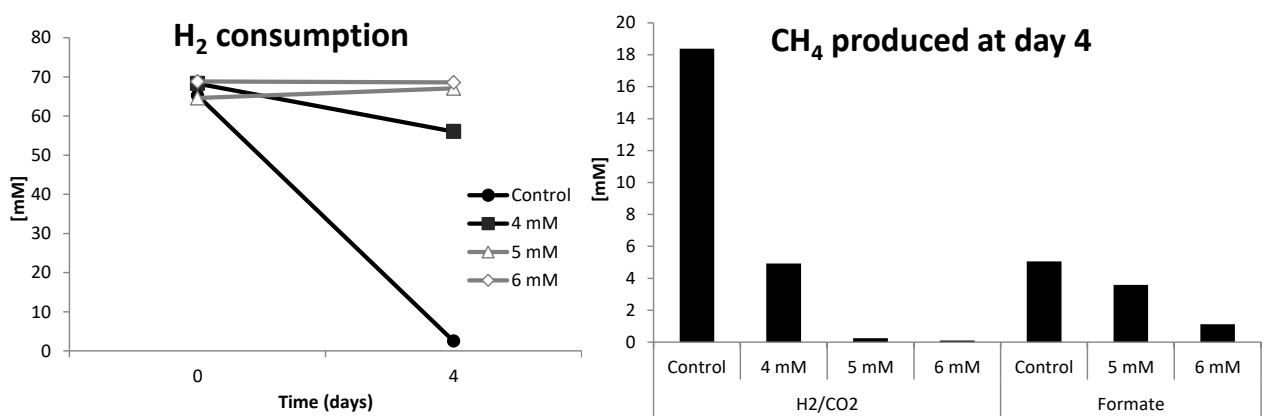

Figure 3.3. Inhibitory effect of sulfide on Methanospirillum hungatei strain JF1. Hydrogen consumption by $M$. hungatei cultures growing at different concentrations of sulfide (left). Methane production of $M$. hungatei cultures in $\mathrm{H}_{2} / \mathrm{CO}_{2}$ or formate under different sulfide concentrations.

To estimate the decay of the syntrophic partner, starvation tests were performed for $M$. hungatei. The archaeon showed to be able to survive for more than 20 days of starvation (Figure 3.4). The results showed that at 19 days of starvation (8 days for cultures growing on $\mathrm{H}_{2} / \mathrm{CO}_{2}$ ) the viability of the cultures was compromised as the delay in methane production shows in Figure 3.4. Nevertheless, we were able to measure methane production in cultures starved up to 39 days.
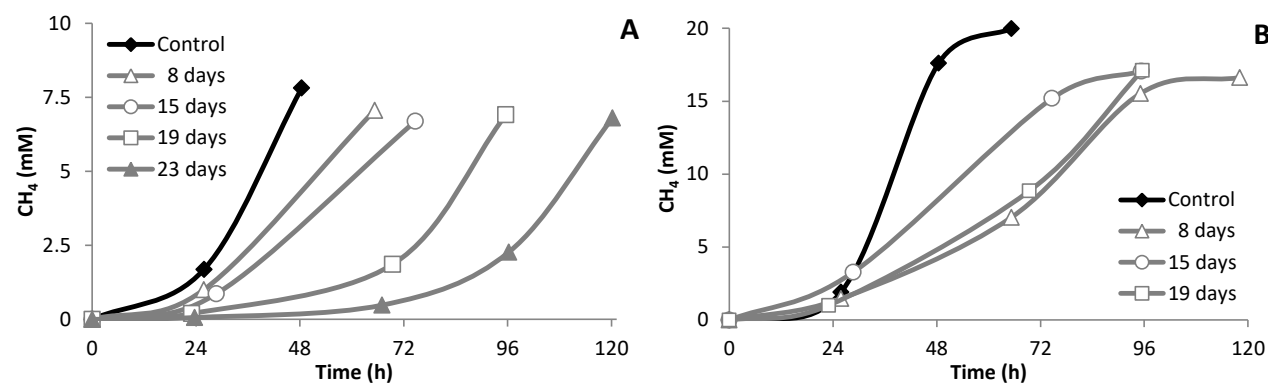

Figure 3.4. Methane production by starved cultures of M. hungatei. Starvation days refer to the length of incubation at $37^{\circ} \mathrm{C}$ without electron donor. Time on $\mathrm{x}$-axis plots the hours after supply of the electron donor: formate $[30 \mathrm{mM}]$ in $\mathrm{A}$ and $\mathrm{H}_{2} / \mathrm{CO}_{2}$ in $\mathrm{B}$.

Despite the resilience of the methanogenic partner and the high percentage of inoculum used in the perturbations of the sulfidogenic cultures, the methane detected was not significant and the low detected values $(<1 \mathrm{mmol} / \mathrm{l})$ were attributed to decay of the methanogens. A final approach was taken aiming to establish a sulfidogenic syntrophic coculture with $S$. fumaroxidans, which was the addition of 
the sulfate reducer, D. desulfuricans G11. D. desulfuricans G11 has previously been used to obtain a sulfidogenic syntrophic coculture with Syntrophobacter wolinii for propionate degradation (Boone and Bryant, 1980).

Data of the substrate consumption and product formation after the perturbations of the sulfidogenic cultures are shown in Table 3.1. Despite the absence of methane, indicating that the syntrophic engagement with methanogens did not occur, higher propionate degradation in treatment $\mathrm{S}+\mathrm{JF} 1$ can be noticed in contrast to S+G11. A possible explanation is that at the moment of the perturbations the sulfide levels were above $10 \mathrm{mM}$, but when $M$. hungatei was added to the media, the sulfide levels dropped by a dilution effect, whereas when $D$. desulfuricans G11 was added, the sulfide levels were maintained since $D$. desulfuricans G11 was also in a medium high in sulfide (Figure 3.2).,

Sulfate-reducing bacteria generally are assumed to be able to deal with high concentrations of sulfide. However, sulfide inhibition in propionate degradation by S. wolinii has been previously reported by (Boone and Bryant, 1980). In that study sulfide concentrations of 5 and $10 \mathrm{mM}$ inhibited propionate degradation by 23 and $51 \%$, respectively. Our results suggest that $S$. fumaroxidans is similarly sensitive to high levels of sulfide. We observed that propionate degradation by $S$. fumaroxidans decreased in those treatments where sulfide levels were kept above $10 \mathrm{mM}$. The process of flushing out the hydrogen sulfide from the bottles in treatments S+N2JF1 and S+N2G11 favoured propionate degradation coupled to sulfate reduction during the first week after perturbations, in contrast to the lower amounts degraded in the second week where the sulfide levels were once again above $10 \mathrm{mM}$.

It was not possible to conclude only by analysing the substrates and products dynamics if $S$. fumaroxidans changed its metabolism to couple propionate oxidation to production of hydrogen and/or formate and if $D$. desulfuricans G11 was able to benefit from that change. Therefore, specific primers designed for the $16 \mathrm{~S}$ rRNA gene of $D$. desulfuricans (Junicke et al., 2014) were used to determine the increase in copy numbers of this bacterium at the end of the experiment after its inoculation during the perturbation events.

The results in Figure 3.3 show the increase over time in copy numbers per ml culture of the 16S rRNA gene of $D$. desulfuricans G11, which verifies the microscopic observations of growth of the bacterium (Data not shown). It should be noticed that the amount of inoculum used for the perturbation in sulfidogenic cultures was larger than the amount used in the methanogenic cocultures. These results reflect the approach taken in the sulfidogenic perturbations where a higher percentage (45\%) of D. desulfuricans G11 was added as inoculum during the perturbation. 


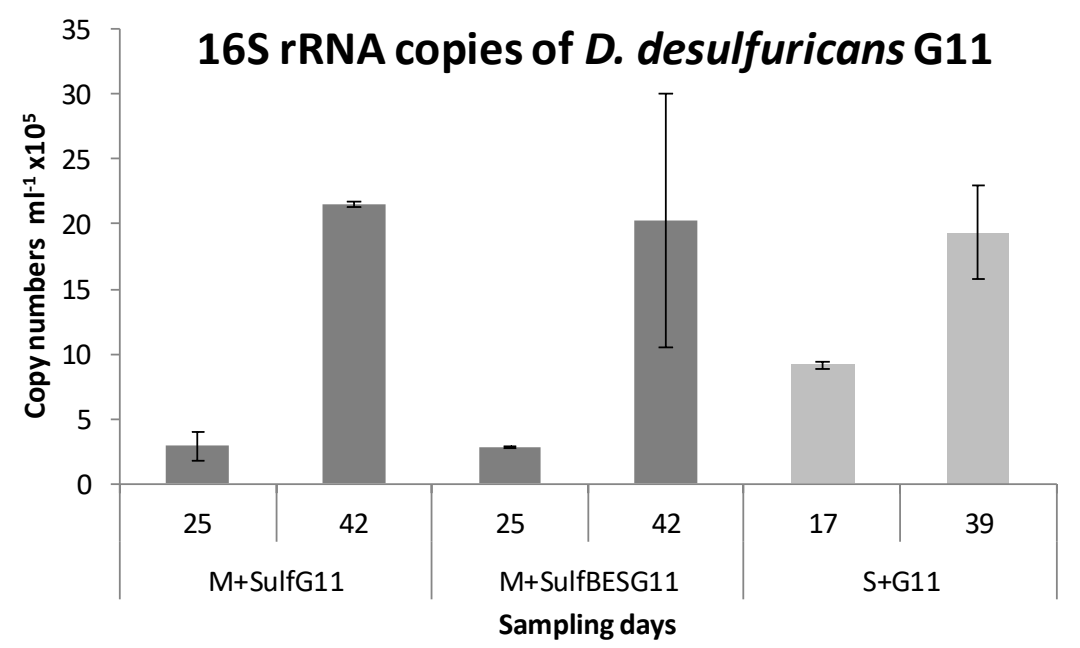

Figure 3.3. 16S rRNA gene abundance of $D$. desulfuricans $\mathrm{G} 11$ at the moment of the perturbations and at the end of the experiments. The dark bars show 16S rRNA copy numbers of $D$. desulfuricans $\mathrm{G} 11$ in cocultures of $S$. fumaroxidans and $M$. hungatei without (M+SulfG11) and with (M+SulfBESG11) addition of bromoethane sulfonate (BES). Light bars show 16S rRNA copy numbers of $D$. desulfuricans G11 in a sulfidogenic culture of $S$. fumaroxidans at a higher proportion than for the cocultures perturbation.

There is a higher increase in copy numbers of D. desulfuricans G11 in the methanogenic cocultures compared to the sulfidogenic culture where G11 barely duplicated its numbers. Propionate oxidation coupled to hydrogen or formate production is endergonic under standard conditions (Equations 1a \& 1b Table 3.2). To make this conversion energetically feasible, the hydrogen and formate concentrations have to be kept in a very low range (Schink, 1997). In the highly active syntrophic methanogenic cocultures, S. fumaroxidans produced formate and hydrogen at high rates while the methanogen kept the levels of those compounds low. Sulfate reducers have a higher affinity for hydrogen than methanogens (Kristjansson et al., 1982; Lovley, 1985). Therefore, once added to the active cocultures, $D$. desulfuricans G11 has competed with the methanogen for hydrogen and formate, which was reflected in the increase in the $16 \mathrm{~S}$ rRNA gene copy numbers. A similar situation occurred in the isolation of $S$. wolinii, where the authors failed to obtain cocultures of $S$. wolinii with $M$. hungatei and D. desulfuricans G11 remained present (Boone and Bryant, 1980). Besides the ability of the sulfate reducer to outcompete the methanogens as a syntrophic partner, the increasing levels of sulfide in M+SulfG11 and the BES added on M+SulfBESG11 also might have contributed to the good proliferation of $D$. desulfuricans G11. Furthermore, syntrophic propionate degradation coupled to sulfidogenesis theoretically yields more energy than when coupled to methane production as can be seen in Equation 1a and 1b of Table 3.3. 
In the sulfidogenic cultures, $D$. desulfuricans G11 scarcely doubled its numbers after inoculation as part of the perturbation (Figure 3.3). Nonetheless, it is interesting that $D$. desulfuricans G11 was able to thrive in this environment. The metabolic activity of $S$. fumaroxidans in sulfidogenic propionate oxidation is very low. Subsequently, a metabolic change, if any, in response to a change in the environment was also expected to be slow. Moreover, as Equation 1a in Table 3.3 show, such metabolic change is not energetically necessary for $S$. fumaroxidans since propionate oxidation coupled to sulfate reduction yields enough energy for growth without a partner. From Equation 2a and 3a we can deduce that for D. desulfuricans G11 it would be beneficial to use the hydrogen and formate produced by S. fumaroxidans, but it is intriguing why the latter bacterium would engage in a syntrophic metabolism while having sufficient sulfate to grow independently. Syntrophic degradation of propionate occurs at the limit of what is thermodynamically possible and requires at least one step with reversed electron transport (Worm et al., 2014). To change from sulfidogenic to syntrophic lifestyle a large metabolic shift would be needed in Syntrophobacter: hydrogenases, formate dehydrogenases and several confurcating complexes need to be switched on. This may be a long process. But when D. desulfuricans G11 is added to an already syntrophic lifestyle it can more easily take over, without much change in the metabolism.

It is still a possibility that the high affinity of $D$. desulfuricans G11 for hydrogen allows this microorganism to scavenge the intermediate hydrogen produced during sulfate reduction by $S$. fumaroxidans. In this case, it would be a parasitic association instead of a syntrophic one. If $S$. fumaroxidans however is genetically driven for syntrophy, a sulfidogenic syntrophic association would be as energetically favourable as a methanogenic one. A genetic predisposition of $S$. fumaroxidans for syntrophy would explain the high proliferation of the alternative hydrogen- and formatescavenger after perturbations in treatment M+SulfG11 and M+SulfBESG11, as well as the growth of the Desulfovibrio in S+G11.

Table 3.3. Gibbs free energy changes of reactions involved in propionate,

formate and hydrogen oxidation coupled to reduction of sulfate and methane production.

\begin{tabular}{|c|c|c|c|}
\hline Equation & & Reactions & $\Delta \mathrm{G}^{\text {ol }}(\mathrm{kJ} / \text { reaction })^{*}$ \\
\hline $1 \mathrm{a}$ & $4 \mathrm{CH}_{3} \mathrm{CH}_{2} \mathrm{COO}^{-}+3 \mathrm{SO}_{4}^{2-}$ & $\rightarrow 4 \mathrm{CH}_{3} \mathrm{COO}+4 \mathrm{HCO}_{3^{-}}+\mathrm{H}^{+}+3 \mathrm{HS}^{-}$ & $-151.3 \mathrm{~kJ}$ \\
\hline $1 b$ & $4 \mathrm{CH}_{3} \mathrm{CH}_{2} \mathrm{COO}+3 \mathrm{H}_{2} \mathrm{O}$ & $\rightarrow 4 \mathrm{CH}_{3} \mathrm{COO}+\mathrm{HCO}_{3}+\mathrm{H}^{+}+3 \mathrm{CH}_{4}$ & $-102.4 \mathrm{~kJ}$ \\
\hline $2 \mathrm{a}$ & $4 \mathrm{H}_{2}+\mathrm{SO}_{4}^{2-}+\mathrm{H}^{+}$ & $\rightarrow \mathrm{HS}^{-}+4 \mathrm{H}_{2} \mathrm{O}$ & $-151.9 \mathrm{~kJ}$ \\
\hline $2 b$ & $4 \mathrm{H}_{2}+\mathrm{HCO}_{3}+\mathrm{H}^{+}$ & $\rightarrow \mathrm{CH}_{4}+3 \mathrm{H}_{2} \mathrm{O}$ & $-135.6 \mathrm{~kJ}$ \\
\hline $3 a$ & $4 \mathrm{HCOO}+\mathrm{H}^{+}+\mathrm{H}_{2} \mathrm{O}$ & $\rightarrow \mathrm{CH}_{4}+3 \mathrm{HCO}_{3}$ & $-130.1 \mathrm{~kJ}$ \\
\hline $3 b$ & $4 \mathrm{HCOO}-+\mathrm{SO}_{4}^{2-}+\mathrm{H}^{+}$ & $\rightarrow \mathrm{HS}+4 \mathrm{HCO}_{3}$ & $-146.7 \mathrm{~kJ}$ \\
\hline
\end{tabular}

* $1 \mathrm{M}, \mathrm{pH} 7.0, \mathrm{~T}=298 \mathrm{~K}$ and a partial pressure of gas of $10^{5} \mathrm{~Pa}$ 


\section{Conclusions}

The addition of sulfate to syntrophic methanogenic cocultures of $S$. fumaroxidans and $M$. hungatei results in a metabolic shift in $S$. fumaroxidans where sulfidogenesis eventually takes over methanogenesis. Although the metabolic shift to sulfidogenesis was observed soon after the addition of sulfate to the media, methanogenic associations remained dominant until the methanogenic partner was affected and inhibited by sulfide. The metabolic shift of $S$. fumaroxidans to sulfidogenesis was not favoured by the inhibition of the methanogen as the treatments with BES indicate.

The methanogenic cocultures ultimately stopped producing methane due to sulfide toxicity to the methanogen. But it is probable that the syntrophic metabolism of $S$. fumaroxidans, where propionate oxidation is coupled to hydrogen and/or formate production, persisted as the increase of the numbers of D. desulfuricans G11 suggests.

The low growth rate of $S$. fumaroxidans in sulfidogenic environments might be attributed to sulfide sensitivity. In environments with sulfide levels above $10 \mathrm{mM}$, propionate degradation considerably drops in comparison to lower sulfide levels. Thus, the inefficiency of $S$. fumaroxidans to degrade propionate in the presence of sulfate could be related to the sulfide toxicity effect on the bacterium.

Although the thriving of $D$. desulfuricans G11 in coculture with S. fumaroxidans in sulfidogenic environments has been verified by qPCR, to know whether $S$. fumaroxidans switched its metabolism from sulfidogenesis to syntrophy still requires further research. 


\section{Supplementary material}

Table S1.1. Electron and carbon balances of the syntrophic cocultures and sulfidogenic cultures of Syntrophobacter fumaroxidans after diverse perturbation events.

\begin{tabular}{|c|c|c|c|c|c|c|c|c|}
\hline \multirow{4}{*}{$\begin{array}{l}\text { Treatments } \\
\text { indicating } \\
\text { perturbations } \\
\text { applied to the } \\
\text { cultures }\end{array}$} & \multicolumn{8}{|c|}{ Syntrophic cocultures of $S$. fumaroxidans and Methanospirillum hungatei } \\
\hline & \multicolumn{4}{|c|}{ Week 1} & \multicolumn{4}{|c|}{ Week 2} \\
\hline & \multicolumn{2}{|c|}{ Electron recovery } & \multicolumn{2}{|c|}{ Carbon balance } & \multicolumn{2}{|c|}{ Electron recovery } & \multicolumn{2}{|c|}{ Carbon balance } \\
\hline & Theoretical & Achieved & Theoretical & Achieved & Theoretical & Achieved & Theoretical & Achieved \\
\hline \multirow{2}{*}{ M+Sulf } & 15.8 & 14.3 & 36.8 & 22.9 & 5.5 & 6.8 & 12.9 & 13.1 \\
\hline & ER \% & 90.5 & CR\% & 62 & ER \% & 123 & $\mathrm{CR} \%$ & 101.4 \\
\hline \multirow{2}{*}{ M+SulfG11 } & 11.4 & 12.4 & 26.7 & 16.4 & 8.6 & 8.3 & 20.1 & 17.3 \\
\hline & ER \% & 108.2 & CR\% & 61.3 & ER \% & 96.5 & CR\% & 86.3 \\
\hline \multirow{2}{*}{ M+SulfBES } & 6.9 & 6.5 & 16.2 & 5.0 & 6.6 & 6.5 & 15.5 & 11.1 \\
\hline & ER \% & 93.2 & CR\% & 31 & ER \% & 98.4 & CR\% & 71.6 \\
\hline \multirow{2}{*}{ M+SulfBESG11 } & 3.7 & 4.8 & 8.5 & 2.1 & 6.2 & 7.4 & 14.5 & 10.4 \\
\hline & ER \% & 131.8 & CR\% & 25.1 & ER \% & 119.4 & CR\% & 71.9 \\
\hline \multirow{2}{*}{ Control } & 14.9 & 16.1 & 34.8 & 35.0 & 7.9 & 8.3 & 18.4 & 19.5 \\
\hline & ER \% & 107.8 & CR\% & 100.8 & ER \% & 104.8 & CR\% & 106.3 \\
\hline \multicolumn{9}{|c|}{ Sulfidogenic cultures of $S$. fumaroxidans } \\
\hline \multirow[t]{2}{*}{ S+JF1 } & 4.81 & 4.0 & 11.22 & 5.91 & 1.69 & 1.4 & 3.94 & 3.17 \\
\hline & ER \% & 83.2 & $\mathbf{C R} \%$ & 52.6 & ER \% & 82.9 & $\mathbf{C R} \%$ & 80.4 \\
\hline \multirow[t]{2}{*}{ S+N2JF1 } & 6.67 & 5.3 & 15.57 & 10.18 & N.A. & N.A. & N.A. & N.A. \\
\hline & ER \% & 78.9 & CR\% & 65.4 & N.A. & N.A. & N.A. & N.A. \\
\hline \multirow[t]{2}{*}{ S+G11 } & 3.71 & 3.3 & 8.66 & 4.57 & 1.66 & 4.0 & 3.88 & 2.85 \\
\hline & ER \% & 88.3 & CR\% & 52.8 & ER \% & 243.2 & CR\% & 73.5 \\
\hline \multirow[t]{2}{*}{ S+N2G11 } & 6.26 & 6.5 & 14.61 & 6.91 & 4.35 & 3.7 & 10.16 & 6.24 \\
\hline & ER \% & 103.3 & CR\% & 47.3 & ER \% & 85.9 & CR\% & 61.5 \\
\hline
\end{tabular}


Mankind has a lot to learn from syntrophy 

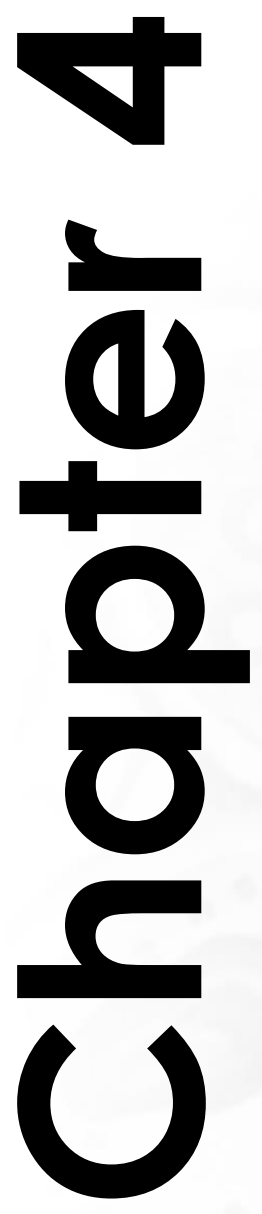


\title{
CHAPTER 4
}

\section{Comparative proteome analysis of propionate degradation by Syntrophobacter fumaroxidans in pure culture and in coculture with methanogens}

\author{
Vicente T. Sedano-Núñez ${ }^{1}$, Sjef Boeren ${ }^{2}$, Alfons J. M. Stams ${ }^{1}$ and Caroline M. Plugge ${ }^{1}$ \\ ${ }^{1}$ Laboratory of Microbiology, Wageningen University and Research, The Netherlands \\ ${ }^{2}$ Laboratory of Biochemistry, Wageningen University and Research, The Netherlands
}

Accepted for publication in Environmental Microbiology 


\begin{abstract}
Syntrophobacter fumaroxidans is a sulfate-reducing propionate-degrading bacterium that grows in syntrophic interaction with methanogens, but a syntrophic interaction with sulfate-reducing bacteria is also possible. We performed a proteome analysis of $S$. fumaroxidans growing with propionate axenically with sulfate or fumarate, and in syntrophy with Methanospirillum hungatei, Methanobacterium formicicum or Desulfovibrio desulfuricans. Special attention was put on the role of hydrogen and formate in interspecies electron transfer (IET) and energy conservation. Formate dehydrogenase Fdh1 and hydrogenase Hox were the main confurcating enzymes used for energy conservation. In the periplasm, Fdh2 and hydrogenase Hyn play an important role in reverse electron transport associated with succinate oxidation. Periplasmic Fdh3, Fdh4 and Fdh5 were involved in IET. The sulfate reduction pathway was poorly regulated and many enzymes associated with sulfate reduction (Sat, HppA, AprAB, DsrAB and DsrC) were abundant even at conditions where sulfate was not present. Heterodisulfide reductases $(\mathrm{Hdr})$, coupled with flavin oxidoreductase (Flox) or a putative hydrogenase (Mvh-p), were abundant. Hdr/Flox was detected in all conditions while Hdr/Mvh-p was exclusively detected when sulfate was available; these complexes most likely confurcate electrons. Our results suggest that $S$. fumaroxidans mainly used formate for electron release and that different confurcating mechanisms were used in its sulfidogenic metabolism.
\end{abstract}

Keywords: Syntrophy, sulfate-reducing bacteria, propionate oxidation, interspecies electron transfer, reverse electron transport, hydrogenases, formate dehydrogenases. 


\section{Introduction}

Syntrophobacter fumaroxidans is a sulfate-reducing deltaproteobacterium able to grow on propionate in syntrophy with methanogens (Harmsen et al., 1998). It can also grow axenically by fermenting fumarate (Stams et al., 1993). To degrade propionate, it requires fumarate or sulfate as electron acceptors, or a $\mathrm{H}_{2}$ - and formate-consuming partner in the absence of an electron acceptor. S. fumaroxidans uses the methylmalonyl-CoA (MMC) pathway to degrade propionate to acetate and $\mathrm{CO}_{2}$ (Plugge et al., 1993). Under standard conditions, propionate oxidation to $\mathrm{H}_{2}$, formate and acetate is an endergonic process. Reducing equivalents at the redox levels of $\mathrm{Fd}_{\text {red, }} \mathrm{NADH}$ and $\mathrm{FADH}_{2}$, are released in the pyruvate, malate and succinate oxidation steps of the pathway, respectively. To keep the pathway functioning, the reduced electron mediators need to be re-oxidized by reducing protons to $\mathrm{H}_{2}$ or $\mathrm{CO}_{2}$ to formate. Consequently, the role of the hydrogen/formate scavenger in the syntrophic association with $S$. fumaroxidans is to maintain $\mathrm{H}_{2}$ and formate at sufficiently low levels so that propionate degradation becomes energetically feasible (Stams and Dong, 1995). The minimal hydrogen partial pressure $\left(\mathrm{pH}_{2}\right)$ that methanogens can maintain is between 1 to $10 \mathrm{~Pa}$ (Thauer et al., 2008). This level is not low enough to overcome the most energy-consuming step in the MMC pathway, the oxidation of succinate to fumarate. To couple this step to proton or $\mathrm{CO}_{2}$ reduction would require a $p \mathrm{H}_{2}$ of $10^{-10} \mathrm{~Pa}$ and a formate concentration below $1 \mu \mathrm{M}$ (Schink, 1997). Therefore, to drive this reaction, the input of metabolic energy is required. An investment of two-thirds of an ATP via a mechanism known as reverse electron transport (RET) has been suggested by some authors (van Kuijk et al., 1998b; Schink and Stams, 2013).

During RET energy is invested in the form of ATP to generate a proton gradient across the membrane which allows succinate oxidation to proceed (Stams and Plugge, 2009). Membrane-associated proteins, such as ferredoxin: $\mathrm{NAD}^{+}$oxidoreductases, cytochromes and periplasmic formate dehydrogenases and hydrogenases, have been reported to be involved in RET (Sieber et al., 2012; Grein et al., 2013). Moreover, novel energy conversion mechanisms have been discovered in anaerobic microorganisms, for instance flavin-based electron bifurcation and its reversal, electron confurcation ( $\mathrm{Li}$ et al., 2008; Buckel and Thauer, 2013; Schink, 2015). Genome analyses of $S$. fumaroxidans revealed membrane associated proteins, such as a fumarate reductase and a Rnf complex, as well as confurcating hydrogenases and formate dehydrogenases possibly involved in energy conservation mechanisms (Müller et al., 2010; Pereira et al., 2011; Plugge et al., 2012; Worm et al., 2014). Subsequently, transcriptomics studies with S. fumaroxidans in syntrophic and axenic cultures showed that a periplasmic formate dehydrogenase (Fdh2) and a hydrogenase (Hyn) play an important role to make the endergonic oxidation of succinate possible (Worm et al., 2011b). 
Moreover, it was found that confurcating hydrogenases and confurcating formate dehydrogenases (Hyd1, Hox and Fdh1) are important energy converting enzymes required for propionate degradation (Worm et al., 2011a; Worm et al., 2011b).

In this study, a comparative proteomic analysis of S. fumaroxidans was made. Cells grown with propionate coupled to fumarate or sulfate reduction, or in syntrophic associations with Methanospirillum hungatei or Methanobacterium formicicum were compared. We aim to elucidate the main metabolic differences in lifestyles by identifying the key proteins used by $S$. fumaroxidans in interspecies electron transfer (IET), reverse electron transport (RET), electron confurcating processes and other energy conservation pathways.

In addition to the known syntrophic interactions of $S$. fumaroxidans with methanogens, our study was extended by including the proteomic profiling of $S$. fumaroxidans in coculture with a non-methanogenic partner. Desulfovibrio desulfuricans has been studied before in cocultures with Syntrophobacter wolinii and S. fumaroxidans as a hydrogen- or formate-scavenger in the oxidation of propionate (Boone and Bryant, 1980; Dong et al., 1994; Sheik et al., 2017). However, the nature of the symbiotic interactions of such cocultures was not properly defined. S. wolinii and S. fumaroxidans are both able to couple propionate oxidation to sulfate reduction instead of proton reduction (Wallrabenstein et al., 1994; van Kuijk and Stams, 1995).

D. desulfuricans is a sulfate reducer that utilizes lactate, ethanol, hydrogen and formate in the presence of sulfate, but not acetate, propionate, butyrate or glucose (McInerney et al., 1979; Sheik et al., 2017). Therefore, a syntrophic relationship with $S$. fumaroxidans, in which hydrogen and formate are produced, would be beneficial for D. desulfuricans. Nonetheless, it is intriguing why Syntrophobacter would engage in a syntrophic association while having sufficient sulfate to grow independently. By comparing the proteomic profile of $S$. fumaroxidans grown in coculture with $D$. desulfuricans with the proteomic profiles of the other known syntrophic lifestyles, and the sulfidogenic condition, we expect to be able to define the symbiotic relationship of $S$. fumaroxidans with $D$. desulfuricans.

Furthermore, in a syntrophic coculture with Methanobrevibacter arboriphilus AZ, D. desulfuricans oxidized formate and provided hydrogen to the methanogenic partner (Dolfing et al., 2008). The proteomic analysis of $D$. desulfuricans growing with hydrogen, formate and in coculture with S. fumaroxidans will reveal further insight into sulfate-reducing syntrophic cocultures. 


\section{Materials and methods}

\section{Organisms and growth conditions}

Syntrophobacter fumaroxidans was grown in pure culture and in cocultures. Syntrophobacter fumaroxidans $\mathrm{MPOB}^{\mathrm{T}}$ (DSM 10017) was cultivated under anoxic conditions in basal medium as described previously (Stams et al., 1993). The medium for the pure cultures was supplemented with $20 \mathrm{mM}$ propionate and $60 \mathrm{mM}$ fumarate. Sulfidogenic cultures were grown on $20 \mathrm{mM}$ propionate and $20 \mathrm{mM}$ sulfate. Cocultures of $S$. fumaroxidans with Methanospirillum hungatei strain JF1 ${ }^{\mathrm{T}}$ (DSM 864) or Methanobacterium formicicum MF' (DSM 1535) were grown with $30 \mathrm{mM}$ of propionate without electron acceptor. A coculture of $S$. fumaroxidans with Desulfovibrio desulfuricans G11 (DSM 7057; (Sheik et al., 2017)) was grown with 20 $\mathrm{mM}$ propionate and $20 \mathrm{mM}$ sulfate. Axenic cultures of $D$. desulfuricans were grown with $20 \mathrm{mM}$ sulfate and $40 \mathrm{mM}$ formate or hydrogen (1.7 atm $\mathrm{H}_{2} / \mathrm{CO}_{2}$ 80:20 vol/vol). All organisms were batch cultured in triplicate at $37^{\circ} \mathrm{C}$ in 1 litre flasks with $550 \mathrm{ml}$ medium under anaerobic conditions provided by a pressurised (172 kPa; $1.7 \mathrm{~atm})$ gas phase of $\mathrm{N}_{2} / \mathrm{CO}_{2}(80: 20$, vol/vol). Growth was monitored by measuring substrate consumption and product formation (propionate, sulfate, methane, acetate, succinate, malate and/or sulfide). Cells were harvested during mid-exponential growth phase. The cultures for the experiment were inoculated with cells from cultures that adapted to these conditions by transferring them at least five times in media with the respective substrates before the start of the experiment.

\section{Harvesting cells and Percoll gradient centrifugation}

Cells were aerobically harvested by centrifugation at $16,000 \mathrm{~g}$ for 16 minutes at $4{ }^{\circ} \mathrm{C}$. The pellet was washed twice with TE buffer (10 mM Tris-HCl, pH 7.5; 1 mM EDTA). Only cells from the syntrophic coculture of $S$. fumaroxidans and $M$. hungatei were separated by Percoll gradient centrifugation (Percoll ${ }^{\circledR}$, Sigma-Aldrich, Missouri, US) as described elsewhere (de Bok et al., 2002a). The separated layers, containing Syntrophobacter cells in the upper layer and Methanospirillum cells in the lower layer, were collected and subjected to Percoll gradient separation a second time. Cells were then washed twice with $10 \mathrm{mM}$ sodium phosphate buffer (pH 7.5).

\section{Protein extraction and SDS-PAGE}

Cells were resuspended in lysis buffer (100 mM Tris-HCl, $\mathrm{pH} 7.5 ; 4 \%$ w/v SDS; 50 $\mathrm{mM}$ dithiothreitol and SIGMAFAST ${ }^{\mathrm{TM}}$ Protease Inhibitor Cocktail Tablet (SigmaAldrich, Missouri, US)), and passed three times through a French press (French ${ }^{\circledR}$ Type Pressure Cell Disrupter, Stansted Fluid Power, Harlow, UK) at $2 \mathrm{MPa}$ (40K cell). Cell debris and undisrupted cells were removed by centrifugation at $18,000 \mathrm{~g}$ for $10 \mathrm{~min}$ at $4{ }^{\circ} \mathrm{C}$. The supernatant was collected in Eppendorf ${ }^{\mathrm{TM}}$ LoBind Protein Microcentrifuge Tubes and stored at $-80{ }^{\circ} \mathrm{C}$. Still in the lysis buffer, proteins were denatured by heating at $95{ }^{\circ} \mathrm{C}$ for 5 minutes. Samples were loaded on a $10 \%$ 
polyacrylamide separation gel (Precise $^{\mathrm{TM}}$ Tris-HEPES Gels, Thermo Scientific, Rockford, US) using the Mini-PROTEAN Tetra Cell (Bio-Rad Laboratories B.V, Veenendaal, The Netherlands). The electrophoresis procedure was according to the precast gels manufacturer's instructions. Gels were stained using Coomassie Brilliant Blue (CBB) R-250. Protein concentration was normalized among triplicates and samples in a qualitative way by analysing the gel pictures taken with G:BOX Chemi XT4 (Syngene, Cambridge, UK) and using the software GeneSys version 1.5.5.0 (GeneTools version 4.03.01).

\section{In-gel trypsin digestion}

In-gel digestion of proteins and purification of peptides was done following a modified version of a previously described protocol (Rupakula et al., 2013). Disulfide bridges in proteins were reduced by covering the gels with reducing solution $(10 \mathrm{mM}$ dithiothreitol, $\mathrm{pH} 7.6$, in $50 \mathrm{mM} \mathrm{NH}_{4} \mathrm{HCO}_{3}$ ), and the gels were incubated at $60^{\circ} \mathrm{C}$ for $1 \mathrm{~h}$. Alkylation was performed in darkness and shaking (100 rpm) for $1 \mathrm{~h}$ by adding $25 \mathrm{ml}$ of iodoacetamide solution (10 mM iodoacetamide in $100 \mathrm{mM}$ Tris- $\mathrm{HCl}, \mathrm{pH}$ 8.0). Gels were thoroughly rinsed with demineralized water in between steps. Each gel lane was cut into 3 slices, and the slices were cut into approximately $1 \mathrm{~mm}^{3}$ cubes and transferred to a separate $0.5 \mathrm{ml}$ protein LoBind tube (Eppendorf, Hamburg, Germany). Enzymatic digestion was done with trypsin sequencing grade (Roche, Mannheim, Germany). $100 \mu \mathrm{l}$ of trypsin solution (5 ng/ $\mu \mathrm{l}$ trypsin in $50 \mathrm{mM}$ $\mathrm{NH}_{4} \mathrm{HCO}_{3}$ ) were added to each tube, and incubated 2 hours at $45{ }^{\circ} \mathrm{C}$ with gentle shaking. To stop trypsin digestion, trifluoroacetic acid (10\%) was added to the supernatant to lower the $\mathrm{pH}$ below 5 . The digested protein mixture was purified and concentrated using an in-house made SPE pipette tip (Lu et al., 2011).To recover hydrophobic peptides, $50 \mu \mathrm{l}$ acetonitrile (vol/vol in $0.1 \%$ formic acid) was passed through the column. Finally, the volume was reduced to $20 \mu \mathrm{l}$ using a SpeedVac concentrator and then adjusted to $50 \mu \mathrm{l}$ with $0.1 \%$ formic acid. Samples were analysed using nLC-MS/MS with a Proxeon EASY nLC and a LTQ-Orbitrap XL mass spectrometer as previously described (Lu et al., 2011).

\section{LC-MS data analysis}

The obtained MS/MS spectra were processed with MaxQuant v. 1.5.2.8. Database with the protein sequences of $S$. fumaroxidans was downloaded from UniProt (www.uniprot.org). The protein database of D. desulfuricans G11 was the draft genome available in our laboratory. An additional dataset with protein sequences of common contaminants (trypsin, human keratins and bovine serum albumin) was included. False discovery rates (FDR) of less than 1\% were set at peptide and protein levels. Modifications for acetylation (Protein N-term), deamidation (N, Q) and oxidation (M) were allowed to be used for protein identification and quantification. All other quantification settings were kept default. Filtering and further bioinformatics and statistical analysis were performed with Perseus v.1.5.3.0. Proteins included in our analysis contain at least two identified peptides of which at 
least one is unique and at least one unmodified. Reversed hits and contaminants were filtered out. Protein groups were filtered to require three valid values in at least one experimental group. Label-free quantification (LFQ) intensities (values normalized with respect to the total amount of protein and all of its identified peptides) were used to analyse the abundance of proteins in the fractions and further statistical comparisons among conditions. LFQ intensities were transformed to logarithmic values base 10 . Missing values were imputed with random numbers from a normal distribution, the mean and standard deviation of which were chosen to best simulate low abundance values close to noise level (Width: 0.3 and downshift 1.8 times). A multiple-sample test (ANOVA) with permutation based FDR statistics (250 permutations, $\mathrm{FDR}=0.01$ and $\mathrm{S} 0=1$ ) was applied to filter significant proteins. Principal component analyses (PCA) were performed with default settings and without category enrichment in components. Z-score normalization in which the mean of each row (where each row is a protein in triplicate and in different conditions) is subtracted from each value and the result divided by the standard deviation of the row was applied before clustering. Hierarchical clustering of rows, using Euclidean distances, produced a heat map representation of the clustered data matrix. Row clusters were automatically defined (100) and exported to a new matrix. Imputed values were then replaced back to missing values and previously defined clusters were displayed in a new heat map. For $D$. desulfuricans the Z-score and hierarchical clustering was done for columns instead of rows in order to compare the most abundant proteins detected in each condition.

\section{Results}

\section{Proteomic overview of S. fumaroxidans and most abundant proteins in all growth conditions}

The genome of $S$. fumaroxidans contains 4,098 protein coding genes (Plugge et al., 2012). Our proteomic analysis accurately identified a total of 813 proteins in the five studied conditions. Of these, 84 were designated as proteins with unknown function. 514 proteins were detected in all the studied conditions. This core proteome represented slightly more than 60\% of all the detected proteins (Supporting information, Fig. S4.1.A). Principal component analysis (PCA) revealed that the protein abundance patterns were reproducible among triplicates of a given growth condition (Supporting information, Fig. S4.1.B). Moreover, it shows that protein patterns of $S$. fumaroxidans differ depending on the electron acceptor or syntrophic partner used, clearly separating syntrophic methanogenic conditions from the axenic proteomic profiles. Statistical analysis indicated that 509 proteins significantly differed in at least one condition. This means that 304 proteins were constitutively produced in the five analysed conditions. 
Total intensity-based absolute quantification (iBAQ) revealed the most abundant proteins produced in the whole analysis. Most of these proteins were involved in the methylmalonyl-CoA pathway, sulfate reduction, electron transfer or energy conservation. Highly abundant proteins under all five conditions included chaperonins (GroEL \& GroES), heat shock proteins and ribosomal proteins. Other abundant proteins had annotated functions involved in protection, signalling, transcription and ferrous ion transport. Rubrerythrins and proteins involved in the biosynthesis of cofactors like iron-molybdenum and molybdopterin were also abundant.

\section{Enzymes of the methylmalonyl CoA pathway}

Previous genomic analyses of $S$. fumaroxidans predicted several genes coding for proteins involved in the MMC pathway (Müller et al., 2010; Plugge et al., 2012). Most of these proteins were abundant in our whole-cell proteome analysis. For those predicted proteins that were not detected, paralogous proteins were found in high levels, which suggests that these proteins have a role in the MMC pathway. For instance, the predicted enzymes for propionate activation (Sfum_3926 to Sfum_3934) and for the conversion of acetyl-CoA to acetate (Sfum_0388-0389, Sfum_0745-0746, Sfum_1278 and Sfum_3070) were not detected in the present study. Nevertheless, three sets of proteins were detected for the five conditions: CoA-A (Sfum_0809-0810), CoA-B (Sfum_0811-0812) and CoA-S (Sfum_1132-1134) (Figure 4.1). The amino acid sequences of these proteins indicate a relationship to coenzyme A transferase family I (InterPro IPR004165) and could therefore be involved in propionate activation and/or acetate formation.

As predicted by previous genome studies (Müller et al., 2010; Plugge et al., 2012), the main protein complex responsible for the oxidation of succinate to fumarate was the membrane bound succinate dehydrogenase SdhABC (Sfum_1998-2000), which was abundant in all conditions. During axenic growth on propionate with fumarate, $S$. fumaroxidans converts propionate to succinate. Then, part of the fumarate in this growth condition is oxidized to acetate (Stams et al., 1993). This conversion is energy dependent, producing reducing equivalents during malate oxidation and pyruvate decarboxylation, and is only possible by coupling it to the energy yielding reduction of fumarate to succinate.

The fumarate reductase FrdABEF (Sfum_4092-4095) complex was detected in higher levels during growth with fumarate. With the exception of a few subunits, the FrdABEF complex was not detected in cells grown with methanogens as expected since fumarate reduction only occurs when fumarate is provided. However, the complex was consistently detected in cells where sulfate was available, particularly in the coculture with $D$. desulfuricans. 


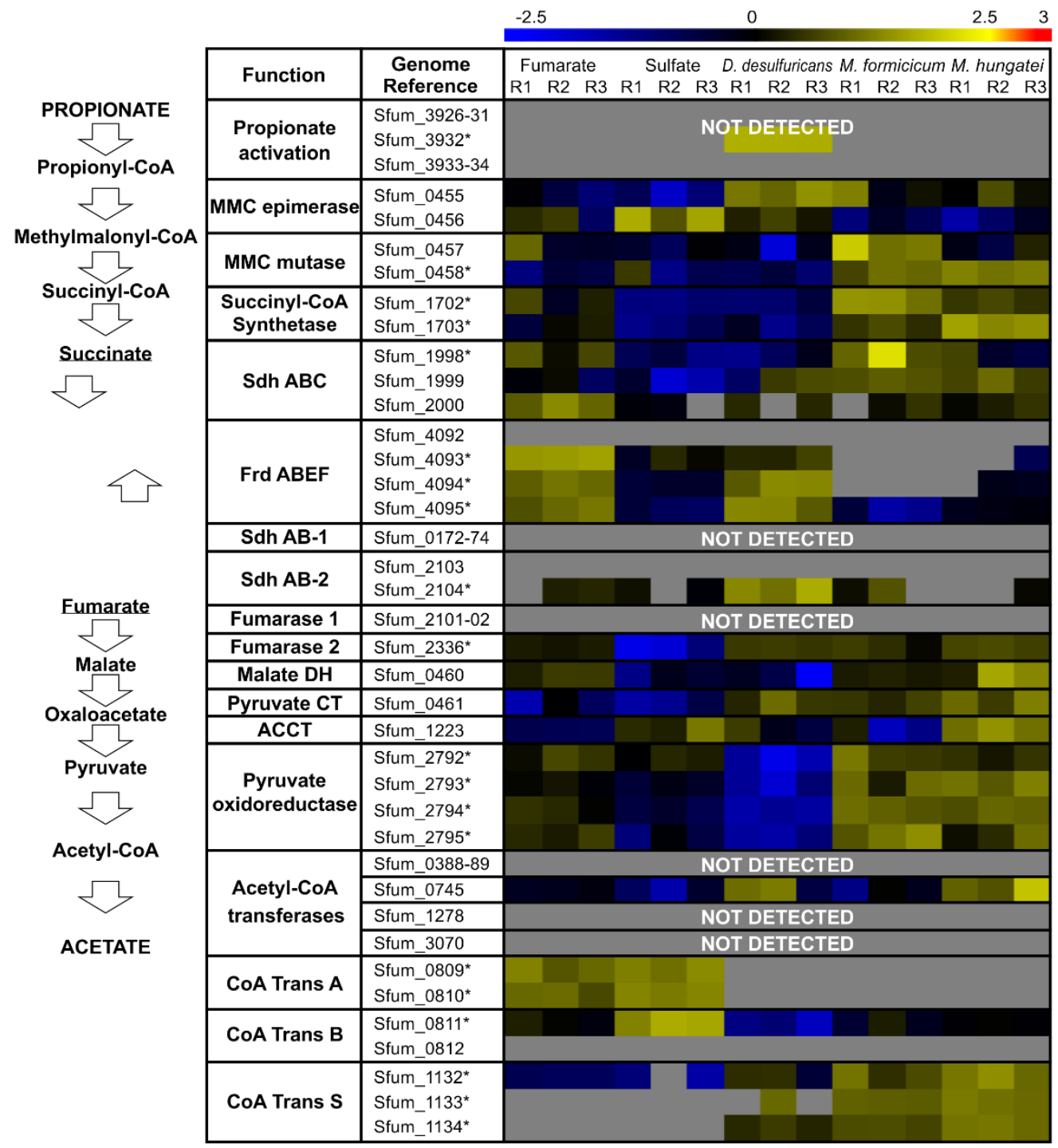

Figure 4.1. Relative expression levels of the proteins used in the methylmalonylCoA pathway by Syntrophobacter fumaroxidans. Protein abundance levels are shown after Z-score normalization. High relative expression is indicated in yellow and low relative expression is indicated in blue. Grey colour means not detected. In the left side the MMC steps are shown levelled to the associated proteins. The rows in the heat map show the detected proteins in five different growth conditions. The columns show from left to right, in triplicates, the electron acceptor used by $S$. fumaroxidans to couple propionate oxidation: fumarate, sulfate and interspecies compounds transferred to: Desulfovibrio desulfuricans G11, Methanobacterium formicicum and Methanospirillum hungatei. $\left(^{*}\right)$ indicates a statistically significant difference in at least one condition. MMC: methylmalonyl-CoA; Sdh: succinate dehydrogenase; Frd: fumarate reductase; DH: dehydrogenase; CT: carboxyltransferase; ACCT: acetyl-CoA carboxyltransferase; CoA Trans: coenzyme A transferase. 
In the genome of $S$. fumaroxidans two additional gene clusters show similarity to succinate dehydrogenases SdhAB-1 (Sfum_0172-0174) and SdhAB-2 (Sfum_21032104). SdhAB-1 was not detected in our study and only the alpha subunit of SdhAB2 showed a similar detection profile to FrdABEF. The predicted fumarase in the gene cluster Sfum_2101-02 was not detected in any condition. Instead, a second fumarase from a non-clustered gene (Sfum_2336) was abundant in all conditions. The amino acid sequence of this second fumarase corresponds to the previously isolated and characterized class I fumarase from S. fumaroxidans (van Kuijk et al., 1996). Although this protein was abundant in all conditions, lower expression levels were measured in sulfate-reducing cells. Finally, methylmalonyl-CoA mutase (Sfum_0458) and succinyl-CoA synthase (Sfum_1702-1703) were significantly more abundant in syntrophically grown cells, while the pyruvate oxidoreductase (Sfum_2792-2795) showed a lower relative expression during growth with Desulfovibrio.

\section{Hydrogenases and formate dehydrogenases involved in electron transfer}

The genome of $S$. fumaroxidans indicates the presence of six formate dehydrogenases and eight hydrogenases. Relative abundance levels of the hydrogenases and formate dehydrogenases produced by $S$. fumaroxidans during propionate degradation under different axenic or cocultured conditions are shown in Figure 4.2. In this figure can be seen that for most of the detected hydrogenases and formate dehydrogenases, the expression levels measured in syntrophic conditions with methanogens were higher than any of the axenic conditions. Of the two predicted periplasmic hydrogenases, Hyn (Sfum_2952-53) was detected in all conditions albeit more abundant during growth with fumarate and with $D$. desulfuricans G11, while Hyd2 (Sfum_0847-48) was not detected in cells that were grown with sulfate.

Figure 4.2. Relative abundance levels of hydrogenases and formate dehydrogenases in Syntrophobacter fumaroxidans during propionate oxidation. Protein abundance levels are shown after Z-score normalization. The detected proteins are shown for five different growth conditions, in triplicates, according to the electron acceptor used by $S$. fumaroxidans to oxidize propionate; from left to right: fumarate, sulfate and interspecies compounds transferred to: Desulfovibrio desulfuricans G11, Methanobacterium formicicum and Methanospirillum hungatei. The colour intensity indicates the degree of protein up- or down regulation where high relative expression is indicated in red and low relative expression is indicated in blue; the grey colour represents not detected. Underlined complex names have been predicted to function as confurcating. Locus tags in bold font indicate the catalytic subunit of the complex. $\left.{ }^{*}\right)$ indicates a statistical significant difference in at least one condition. 


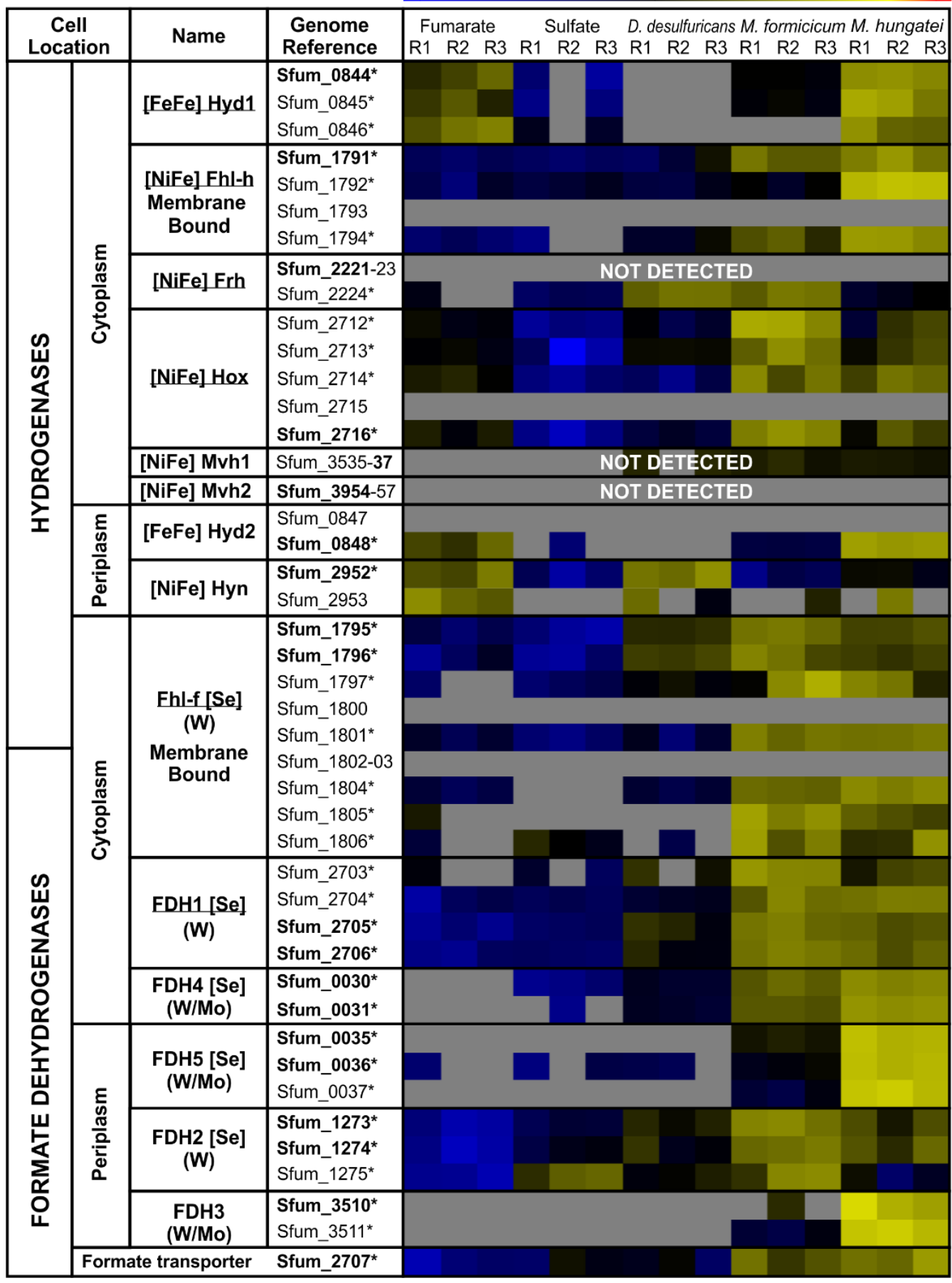


S. fumaroxidans has two [NiFe]-hydrogenases (MvhADG) associated with HdrABC clusters (Mvh1, Sfum_3535-3537 and Mvh2, Sfum_3954-3956). None of these proteins, or the neighbouring $\mathrm{Hdr}$, were found in our analysis. For the Frh complex (Sfum_2221-24), only the subunit containing the FAD and NAD+-binding oxidoreductase domain was detected. Therefore, this protein was classified as not detected. Of the three cytoplasmic hydrogenases detected, Hox (Sfum_2712-16) and Fhl-h (Sfum_1791-94) were present in all conditions. Lastly, Hyd1 (Sfum_0844) was more abundant in syntrophically grown cells and cells grown with propionate and fumarate, but not when sulfate was present.

The three periplasmic formate dehydrogenases (Fdh2, Fdh3 and Fdh5) from $S$. fumaroxidans were abundant during growth in syntrophy with $M$. hungatei. However, for syntrophic growth with $M$. formicicum the detection levels of Fdh5 (Sfum_0035-37) and Fdh3 (Sfum_3509-11) were significantly lower. Fdh3 was not detected in axenic conditions or in the coculture with $D$. desulfuricans, and Fdh5 was scarcely detected in such conditions.

Cytoplasmic Fdh1 (Sfum_2703-06) and periplasmic Fdh2 (Sfum_1273-75) were the most abundant formate dehydrogenases in all conditions. Moreover, significantly higher levels were measured during syntrophic growth. The membrane bound Fhl-f (Sfum_1795-1806) was abundant in syntrophically grown cells but showed a lower relative expression during axenic growth. Fdh4 (Sfum_0030-01) had very high relative abundance levels in syntrophic cultures. However, Fdh4 was not detected in the pure culture with fumarate, while only the lowest limits of detection were measured in sulfidogenic growth. The formate transporter (Sfum_2707) was detected in all conditions but more abundant in methanogenic cultures.

\section{Redox proteins involved in dissimilatory sulfate reduction}

A set of proteins required for dissimilatory sulfate reduction have previously been predicted in the genome of $S$. fumaroxidans (Pereira et al., 2011). Sulfate adenylyltransferase (Sat), proton-translocating pyrophosphatase (HppA), APS reductase (AprAB), dissimilatory sulfite reductase (DsrAB) and DsrC complexes were among the most abundant proteins in all growth conditions. In contrast, neither of the two sulfate transporters (Sfum_0271 \& Sfum_0653) predicted in the genome was detected in the analysis. Two periplasmic subunits of the QrcABCD complex (QrcB: Sfum_0610 and QrcC: Sfum_0609) were detected in all conditions and more abundant in syntrophic cultures (Figure 4.3). However, the subunit QrcA (Sfum_0611) a membrane-associated multihaem cytochrome $c$, was not detected. Sfum_4047 is the only other gene in S. fumaroxidans genome coding for a membraneanchored multihaem cytochrome $c$. The product of this gene was also detected in all conditions and more abundant in the cocultures with $M$. hungatei and $D$. desulfuricans. 


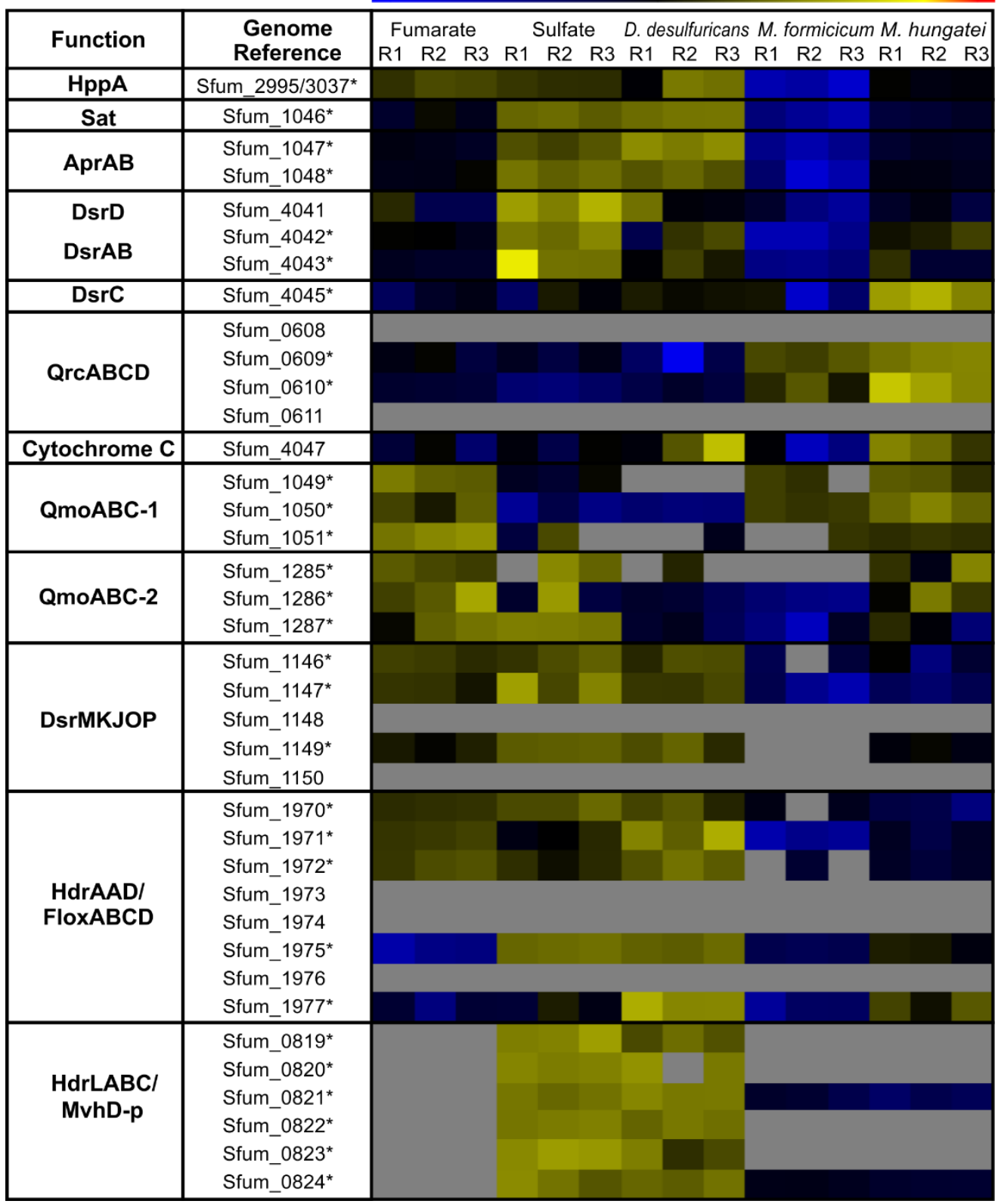

Figure 4.3. Relative abundance levels of proteins involved in sulfate reduction in Syntrophobacter fumaroxidans. Abundance levels after shown after Z-score normalization. The columns show in triplicates, the electron acceptor used by $S$. fumaroxidans to couple propionate oxidation, from left to right: fumarate, sulfate and interspecies compounds transferred to: Desulfovibrio desulfuricans G11, Methanobacterium formicicum and Methanospirillum hungatei. High relative expression is indicated in red and low relative expression is indicated in blue. Grey colour means not detected. (*) indicates a statistical significant difference in at least one condition. 
The genes coding for the trimeric complex QmoABC (Sfum_1049-1051) are well conserved in all known sulfate-reducing bacteria (SRB) and are commonly located in a sat-aprAB-qmoABC cluster (Pereira et al., 2011). Surprisingly, the products of these genes were more abundant in cells grown with fumarate and in syntrophy than in cells grown with sulfate. However, a second QmoABC (Sfum_1285-87) was detected in the proteome in all conditions. This complex was more abundant in cells grown axenically and in the coculture with $M$. hungatei. Similarly, the principal subunits of the DsrMKJOP (Sfum_1146-1150) complex were found in all conditions but more abundant in axenic conditions and in the coculture with $D$. desulfuricans.

Heterodisulfide reductases $(\mathrm{Hdr})$ are enzymes present in methanogens and perform the reduction of CoM-S-S-CoB heterodisulfide to CoM-SH and CoB-SH (Hedderich et al., 2005). Although the substrate of these enzymes CoM-S-S-CoB heterodisulfide has only been found in methanogens, the high number of similar proteins (heterodisulfide reductases-like) in SRB has been emphasized in several genome analyses (McInerney et al., 2007; Strittmatter et al., 2009; Pereira et al., 2011; Grein et al., 2013). Moreover, related enzymes have been purified from other nonmethanogenic archaea (Mander et al., 2004). An Hdr was detected in the proteome analysis of $S$. wolfei (Sieber et al., 2015), suggesting that the presence in the genome and production of such an enzyme complex is not dependent of a sulfate-reducing lifestyle, but rather to microorganisms specialized in low energy metabolism. Two of the five predicted heterodisulfide reductases-like enzymes in S. fumaroxidans were detected in this study, one associated with a Flox complex Hdr/Flox (Sfum_19701977) and the other with a putative methyl viologen hydrogenase $\mathrm{Hdr} / \mathrm{Mvh}-\mathrm{p}$ (Sfum_0819-0824). The Flox section of Hdr/Flox is produced in all conditions. Hdr/Mvh-p was abundant when sulfate was present whereas only the subunits containing FAD/NAD-binding domains were detected in syntrophic cultures. The fifth heterodisulfide reductase-like found in the genome of $S$. fumaroxidans is associated with a pyruvate:Fd oxidoreductase, HdrAL/POR (Sfum_0012-0018); this complex was not detected.

\section{Other proteins involved in energy conservation}

The principle of electron bifurcation was originally proposed for a butyryl-CoA dehydrogenase/electron transferring flavoprotein complex (Bcd-Etf) in Clostridium kluyveri (Li et al., 2008). Since then three more flavin-containing complexes capable of electron bifurcation in bacteria and archaea have been described: [FeFe]hydrogenases (Hyd), transhydrogenases (NfnAB) and [NiFe]hydrogenase/heterodisulfide reductases (MvhADG-HdrABC) (Schut and Adams, 2009; Kaster et al., 2011b; Huang et al., 2012; Buckel and Thauer, 2013).

Although S. fumaroxidans is not able to grow on butyrate or crotonate, complexes similar to Bcd/Etf have been predicted from the genome. The acyl-CoA subunit 
(Sfum_1371) of one of these complexes was abundant in all conditions, while the Etf subunits (Sfum_1372 and Sfum_1373) were detected in lower levels, and the beta subunit was not detected at all in cells grown in cocultures. A second Etf complex from genes Sfum_0106 and Sfum_0107 was abundant in all conditions at similar levels than the acyl-CoA subunit from gene Sfum_1371. (Supporting information, Fig. S4.2) Two additional paralogs coding for Acyl-CoA/Etf complexes were found in the genome (Sfum_3686-88 and Sfum_3929-3931), but not detected in our proteomic analysis. Finally, NfnAB (Sfum_2150-2151), another electron-bifurcating iron-sulfur flavoprotein commonly present in genomic analyses of sulfate reducers was exclusively detected during growth with fumarate.

\section{Proteome generalities of Desulfovibrio desulfuricans}

At the time of our analysis, the genome of Desulfovibrio desulfuricans G11 was not available from the common databases. Therefore, we used a draft genome available in our laboratory. Currently the genome of Desulfovibrio desulfuricans DSM 7057 is freely accessible and counts with 3,020 protein-coding genes (Sheik et al., 2017). Our proteome analysis successfully detected 827 proteins among the three growing conditions. The core proteome of $D$. desulfuricans consists of 344 proteins detected in all studied conditions (Supporting information, Fig. S4.3.A). Only 346 proteins were detected in cells grown in coculture with $S$. fumaroxidans, while the cells growing with hydrogen or formate yielded more than 800 proteins each.

Differences in the proteome composition were explored using principal component analysis (PCA) (Supporting information, Fig. S4.3.B). The first principal component ( $\mathrm{PC} 1 ; 88.5 \%$ of total variance) clearly separates growth in coculture from axenic growth in formate or hydrogen. However, PC1 did not establish a difference between growth on hydrogen or on formate. The second principal component mainly differentiates the two axenic proteomic profiles, albeit PC2 accounts only for $3.8 \%$ of the variability of the data.

Although fewer D. desulfuricans proteins were detected in cells grown in coculture with $S$. fumaroxidans, it was possible to recuperate the most abundant proteins in such condition (Supporting information, Fig. S4.4). Among these, we found a periplasmic formate dehydrogenase (FDH3; DsvG11_3108-3110) and a periplasmic [NiFe]-hydrogenase (Hyd2; DsvG11_2079-2080) (Supporting information, Fig. S4.5). A cytoplasmic formate dehydrogenase (FDH1; DsvG11_1734-1736) on the other hand, was detected only in cells grown with formate, while a periplasmic [FeFe]-hydrogenase (Hyd1; DsvG11_0345-0346) was found in both axenic conditions. We predict from our draft genome of $D$. desulfuricans another [NiFe]-hydrogenase with a cytochrome type-b (Hyd3; DsvG11_1724-1726) and a putative confurcating formate dehydrogenase (FDH2; DsvG11_2896-2899), but neither of these enzymes, nor the formate transporter (DsvG11_0600), were detected in the proteomic results. 
Other periplasmic and membrane bound proteins were abundant in cells grown in the coculture, for instance cytochrome c552 (DsvG11_0693) and an outer-membrane protein (DsvG11_1704).

\section{Discussion}

The majority of the most abundant proteins detected in this study were involved in major processes such as propionate degradation, sulfate reduction, electron transfer, and energy conservation. Other abundant proteins, such as heat-shock proteins, chaperonins, histones and transporters, emphasize the importance of protection, transport and stabilization of diverse macromolecules in the cell. These proteins have previously been reported as highly abundant in several proteomic analyses and identified as common stress-induced molecules required for normal cell growth (Hemmingsen et al., 1988; Lu et al., 2007; Mancuso et al., 2012; Sieber et al., 2015).

\section{Energy-dependent succinate oxidation in MMC}

For propionate degradation with fumarate, S. fumaroxidans requires a fumarate reductase, whereas to oxidize propionate with sulfate, or in syntrophy, a succinate dehydrogenase is needed. The high levels of the fumarate reductase (FrdABEF) in cells grown with propionate and fumarate reflects the reduction of fumarate in this lifestyle. However, the abundance of this complex in cells growing with sulfate and in coculture with $D$. desulfuricans can only be explained by a reversible performance to succinate oxidation, since no succinate was accumulated in those conditions. Fumarate reductases and succinate dehydrogenases are functionally and structurally related enzymes (Mattevi et al., 1999). The membrane bound SdhABC of $S$. fumaroxidans has previously been purified, characterized and showed activity in both directions, fumarate reduction and succinate oxidation (van Kuijk et al., 1998a). However, FrdABEF has not been purified and as such could not be tested for a reversible activity. Transcription experiments reported that FrdABEF was upregulated (>2 log ratio) when fumarate was the electron acceptor in contrast with the gene transcription of cells gown in syntrophic cocultures with M. hungatei (Worm, 2010). Interestingly in such study FrdABEF was also up-regulated in cells grown with sulfate as the electron acceptor and down-regulated in cells cocultured with $M$. formicicum. Our proteomic study confirms the high expression levels of FrdABEF in propionate plus fumarate cultures. Moreover, FrdABEF was also present in conditions where propionate was oxidized with sulfate and in coculture with $D$. desulfuricans. These results might suggest a reversible function of the fumarate reductase FrdABEF towards succinate oxidation. Nevertheless, although in in-vitro analysis the reversible activity of enzymes is possible, in vivo the enzymes are usually dedicated to one physiological function. Besides S. fumaroxidans has a succinate dehydrogenase (SdhABC) for succinate oxidation. A more likely possibility is that fumarate reduction occurred in the sulfidogenic condition. To pull the 
oxidation of succinate towards the formation of fumarate, hydrogen and formate, these products have to be efficiently removed. To maintain the levels of fumarate low, the fumarase (Sfum_2336) has to convert fumarate efficiently to malate. This process is very important, and as such fumarase is one of the most abundant proteins in S. fumaroxidans. However, cells grown with sulfate show the lowest expression levels of this enzyme (Figure 4.1). It might be that if fumarate is not removed efficiently in sulfate-grown cells, the bacteria start to produce FrdABEF.

\section{Hydrogen and formate in IET and RET}

During syntrophic growth, S. fumaroxidans needs to transfer electrons via hydrogen and/or formate to a syntrophic partner. It has long been speculated that formate plays a more important role than hydrogen as an electron carrier in the syntrophic associations of this bacterium with methanogens (de Bok et al., 2002a; de Bok et al., $2002 \mathrm{~b}$ ). Although slightly higher levels were measured in the formate transporter during syntrophic growth over the axenic conditions, S. fumaroxidans must rely on other mechanisms to transfer formate. Three formate dehydrogenases (Fdh2, Fdh3 and Fdh5) contain a twin-arginine translocation (Tat) pathway conserved site, which points to the translocation of these proteins across the cytoplasmic membrane. Fdh3 and Fdh5 were detected only in syntrophically grown cells, while Fdh2 was detected in all conditions, but was more abundant during syntrophic growth. This suggests that periplasmic Fdh3 and Fdh5 are complexes specialized in transferring formate to the syntrophic partner, while Fdh2 is broadly used for energy conservation purposes as part of the reverse electron transport mechanism, possibly coupled to SdhABC or FrdABEF (Fig. S4.6).

Among the cytoplasmic formate dehydrogenases, Fdh1 is homologous to the bifurcating [FeFe]-hydrogenase of Thermotoga maritima (Schut and Adams, 2009). Furthermore, it contains a conserved site coding for a $51 \mathrm{kDa}$ subunit of a NADH:ubiquinone oxidoreductase which makes this protein a very plausible candidate for a confurcating-type of formate dehydrogenase. Fdh1 was detected in all conditions and higher levels were detected in syntrophic conditions. Similarly, the membrane associated Fhl-f was also detected in all conditions and more abundant in syntrophically grown cells. The ubiquitous detection of Fdh1 and Fhl-f indicates that their role is not restricted to IET, but that these complexes are essential for energy conservation and formate/hydrogen interconversion during propionate degradation. On the other hand, Fdh4 was not detected in cells grown with fumarate, scarcely detected in cells grown with sulfate and highly abundant in methanogenic conditions. This led us to speculate that Fdh4 has an exclusive role in IET. Furthermore, the genes coding for Fdh4 are located upstream in the genome of the periplasmic Fdh5 operon. Considering these observations, we propose that these neighbouring genes coding for cytoplasmic and periplasmic formate dehydrogenases are used mainly for interspecies formate transfer. Thus Fdh3, Fdh4 and Fdh5 seem to form a set of 
formate dehydrogenases used by $S$. fumaroxidans to transfer electrons to the syntrophic partner. It is conceivable that these formate dehydrogenases contain a molybdenum catalytic core (Mo-FDH) in contrast to Fdh1 and Fdh2 whose structure has been characterized and were shown to possess only tungsten-containing active sites (W-FDH) (de Bok et al., 2003). Further biochemical analysis of these formate dehydrogenases will give insight of the role of molybdenum in IET mechanisms in methanogenic environments (Plugge et al., 2009; Worm et al., 2011a).

Only five of the eight predicted hydrogenases of $S$. fumaroxidans were detected in the present analysis. Of the two periplasmic hydrogenases, Hyn was more abundant in cells grown with propionate and fumarate and in coculture with $D$. desulfuricans. Hyn has been proposed to be involved in reverse electron transport coupled with FrdABEF for fumarate reduction or SdhABC for succinate oxidation (Worm et al., 2011b). Considering the high levels of Hyn and FrdABEF in the coculture with $D$. desulfuricans, we suggest that indeed Hyn is involved in RET with FrdABEF, whether reducing fumarate in fumarate conditions or reversibly oxidizing succinate in the coculture with $D$. desulfuricans (Fig. S4.6).

Of the three cytoplasmic hydrogenases detected, Hox and Fhl-h, which were detected in all conditions, were more abundant in cells grown with the methanogens. Hox is most probably a confurcating hydrogenase involved in energy conservation. The membrane-bound Fhl-h on the other hand, together with Fhl-f might be involved in a cytoplasmic hydrogen-formate interconversion during syntrophic growth to control electron release. Finally, the genes coding for Hyd1 and Hyd2 are adjacent in the genome, the products of these genes are produced only in the presence of fumarate and during syntrophic growth but not when sulfate was available. This might be due to the exclusive use of other confurcating energy-conserving complexes in sulfidogenic conditions that are also coupled to $\mathrm{H}_{2}$ formation, for instance Mvh-p/Hdr.

Although formate formation seems to prevail in the syntrophic lifestyle of $S$. fumaroxidans, our results indicate that hydrogen, via Hyd1, Hyd2, Hox and Hyn also plays an important role in energy conservation by RET. During growth with fumarate, when IET is not required, these hydrogenases were detected in higher abundance than any of the formate dehydrogenase in such growth condition.

\section{Energy conservation mechanisms in the sulfate-reducing metabolism}

All the proteins necessary for sulfate reduction in $S$. fumaroxidans were abundant in this analysis, with the intriguing exception of the sulfate transporters that were not detected. In order to activate sulfate by sulfate adenylyltransferase, sulfate has to be transported into the cell. Therefore, another mechanism for transport of sulfate across the membrane must be used by $S$. fumaroxidans. Several transporters and unknown proteins were among the most abundant proteins in this study, it is 
possible that some of them could have played a role in the import of sulfate to the cytoplasm.

The abundance of HppA, Sat, Apr and DsrAB in our proteomic analysis in conditions where sulfate reduction was not observed indicates that the sulfate reduction pathway is not strictly regulated in S. fumaroxidans. However, all these enzymes were significantly more abundant in conditions where sulfate was available, indicating sulfidogenic activity in cells grown with sulfate and with $D$. desulfuricans. Similarly, for complexes such as Qmo-2, DsrMKJOP and Hdr/Flox it is possible to observe an up-regulation in axenic conditions and in some cases in coculture with $D$. desulfuricans, while for Qrc and Qmo-1 higher levels are observed in syntrophically grown cells. These observations suggest that the use of these complexes in electron transfer is not constrained to a sulfidogenic lifestyle, and that they could for instance transfer electrons to periplasmic formate dehydrogenases for IET or to the FrdABEF for RET.

Quinone reductase complexes (QrcABCD) are involved in the reduction of the quinone pool in D. vulgaris Hildenborough. Furthermore, it was shown that QrcABCD is reduced by periplasmic hydrogenases and formate dehydrogenase via the cytochrome c3 (subunit A of the complex) (Venceslau et al., 2010). Although in $D$. vulgaris the described role of QrcABCD is to reduce menaquinone with electrons gained from hydrogen or formate oxidation during sulfate reduction, we speculate that a reverse process is feasible. In $D$. desulfuricans G20, a mutant lacking the $q r c B$ gene was unable to grow with $\mathrm{H}_{2}$ or formate as electron donor, while it grew similarly as the parent strain with lactate (Li et al., 2009). Moreover, this mutation also inhibited syntrophic growth with a methanogen in lactate (Li et al., 2009). The higher levels of the QrcABCD of $S$. fumaroxidans in cells grown in syntrophy might be explained by its involvement in electron transfer to the periplasmic formate dehydrogenases Fdh3 and Fdh5 (Figures 4.2 and 4.3).

Direct electron transfer from QmoABC to AprAB to facilitate the reduction of sulfate to sulfite has been reported in Desulfovibrio desulfuricans (Pires et al., 2003; Pereira, 2008; Duarte et al., 2016). In Syntrophobacter, the higher expression levels of the two Qmo complexes in cells grown with fumarate might be due to the use of this membrane bound complex in transferring electrons to FrdABEF for RET. FrdABEF lacks a transmembrane subunit, therefore it has been speculated that it receives electrons from menaquinone via cytochrome $b$ and cytochrome b:quinone oxidoreductases (Müller et al., 2010), however these cytochromes were not detected in our study.

DsrMKJOP is another highly conserved membrane complex in SRB (Rabus et al., 2015). In many Gram-positive SRB only the cytoplasmic-facing DsrMK genes are present, suggesting that this is the minimal functional module (Pereira et al., 2011). 
Although in S. fumaroxidans the complete gene set of DsrMKJOP is present, only the essential subunits (DsrMK), and the periplasmic DsrO were detected in our proteomic study. In the heat map shown in Figure 4.3 the expression profile of DsrMKO is similar to that of the Hdr/Flox complex. If Hdr/Flox is used in all conditions to confurcate electrons as will be discussed below, DsrMKO might be involved in electron transfer with this complex.

HdrABC/FloxABCD, a novel NADH dehydrogenase/heterodisulfide reductase widespread in anaerobic bacteria has been proposed to be involved in flavin-based electron bifurcation in D. vulgaris Hildenborough (Ramos et al., 2015). The Flox proteins (Sfum_1970-1973) of the Hdr/Flox of S. fumaroxidans were constitutively present in all the conditions. Nevertheless, the Hdr-like complex in the Hdr/Flox cluster have a composition different to the canonical HdrABC. For instance, HdrBC is replaced by the cysteine-rich containing HdrD (Sfum_1969), which was not detected in our analysis. Furthermore, two $h d r$ A genes are present (Sfum_1974 \& Sfum_1977), but only the product of Sfum_1977 was detected. Hdr/Flox could be another confurcating system used by $S$. fumaroxidans to re-oxidize NADH during propionate degradation, and possibly involved in recycling $\mathrm{NAD}^{+}$during the partial reduction of fumarate. However, the conformational changes mentioned above might imply functional differences that need to be further investigated.

For the Hdr/Mvh-p complex, the $h d r A B C$ genes (Sfum_0819-0821) are next to genes coding for a pyridine nucleotide-disulphide oxidoreductase comprising an $\mathrm{HdrL}$ protein (Sfum_0824). HdrL is a large protein containing HdrA and one or two NADH binding domains (Strittmatter et al., 2009; Pereira et al., 2011). An MvhD protein is encoded in Sfum_0823, but the catalytic hydrogenase subunit MvhA is not present. The amino acid sequence of Sfum_0822 indicates a relationship to coenzyme F420 hydrogenase (InterPro, December 2017). However, a BlastP search of the amino acid sequence resulted in significant alignments with sequences of formate dehydrogenases in other SRB. We can only speculate if this Hdr/Mvh-p complex uses hydrogen or formate, but the high detection levels of the complete complex imply an important function in the sulfate-reducing metabolism.

Hdr/Mvh-p was detected only in conditions where sulfate was present, axenically or in the presence of $D$. desulfuricans. The soluble complex MvhADG/HdrABC has been shown to perform flavin-based electron bifurcation in methanogens (Thauer et al., 2008; Kaster et al., 2011b). We speculate that Hdr/Mvh-p is preferred when sulfate is available, over the confurcating hydrogenase Hyd1 which in turn was highly abundant in cells grown with fumarate as electron acceptor and in syntrophy, but not detected when sulfate was in the medium (Figure 4.2). The reason for the preference of $\mathrm{Hdr} / \mathrm{Mvh}$ under sulfidogenic conditions is unclear. However, it could be related to the substrates used by this complex. The MvhADG/HdrABC in methanogens uses $\mathrm{H}_{2}$ to reduce ferredoxin and heterodisulfide (Kaster et al., 2011b). 
It is possible that the exclusive high levels of $\mathrm{Hdr} / \mathrm{Mvh}-\mathrm{p}$ in our sulfidogenic conditions correspond to the need of reduction of the so called "bacterial heterodisulfide” DsrC (Venceslau et al., 2014).

It has been suggested (Venceslau et al., 2014), that the protein DsrC could serve as a redox hub, linking oxidation of several substrates to sulfate reduction. Our results with S. fumaroxidans show DsrC as one of the most abundant proteins present in all conditions and significantly more abundant in syntrophy with $M$. hungatei. The recent discoveries point to the role of DsrC as an electron carrier interacting with DsrAB, DsrMKJOP, Hdr/Flox and Hdr/Mvh, but it could also connect other enzyme complexes like the fumarate reductase FrdABEF in $S$. fumaroxidans, which in turn would also explain the detection of FrdABEF in cells grown with sulfate.

\section{Proteomic profiling of Desulfovibrio desulfuricans}

The low amount of $D$. desulfuricans proteins detected from cells grown in coculture with $S$. fumaroxidans can be the result of low biomass in such condition. From microscopic observations we know that the ratio of $S$. fumaroxidans to $D$. desulfuricans was 2:1 (data not shown). Although normalization of the data performed with MaxQuant allowed us to compare the detected proteins with the other growth conditions where more proteins were identified, we rather focused in analysing the most abundant proteins detected in the coculture condition.

The abundance of the periplasmic Hyd2 and periplasmic FDH3 in cells grown with S. fumaroxidans indicates that interspecies electron transfer carried by formate and hydrogen was taking place in the coculture. The abundance of the proteins involved in sulfate reduction confirm that $D$. desulfuricans was actively reducing sulfate for which it certainly needed electron donors which could only come from $S$. fumaroxidans in such growth condition. This shows a remarkable metabolic tendency of $S$. fumaroxidans to engage in syntrophic interactions.

\section{Conclusions}

This study shows the importance of formate as electron carrier in IET and RET during syntrophic and axenic growth of Syntrophobacter fumaroxidans. S. fumaroxidans utilizes a specific set of enzymes (Fdh3, Fdh4 and Fdh5) to transfer electrons to the syntrophic partner. Previous isolation and characterization of Fdh1 and Fdh2 have revealed only tungsten-containing active sites (W-FDH). Biochemical analysis of the three above mentioned formate dehydrogenases could provide insight of the role of molybdenum-dependent formate dehydrogenases in syntrophic growth.

Fdh2 and Hyn are the periplasmic enzymes used by $S$. fumaroxidans to recycle hydrogen and formate during RET. While Fdh2 is mainly coupled to Sdh during 
succinate oxidation, Hyn seems to be coupled to Frd for fumarate reduction in propionate plus fumarate but also for succinate oxidation in other growth conditions.

Although the sulfate-reducing metabolism is poorly regulated, the abundance of membrane-bound complexes like Qrc, Qmo and DsrMKJOP, consistently found in all conditions, as well as the absence of cytochromes in the present study (only 2 cytochromes detected from 8 predicted in the genome), indicates that those membrane-bound complexes might play a role in the transfer of electrons between cytoplasmic enzymes and the periplasmic formate dehydrogenases and hydrogen dehydrogenases.

Hdr/Mvh-p is the most abundant putatively confurcating system in sulfidogenic conditions, possibly because of its probable connection to DsrC, an electron hub in sulfidogenic metabolism.

The proteomic profiles of both bacteria in the coculture of $S$. fumaroxidans with $D$. desulfuricans gives insight in the metabolic flexibility of $S$. fumaroxidans. Results showed a proteomic profile of $S$. fumaroxidans in which sulfate reduction took place, while energy conservation and IET mechanisms were also used similarly as in the syntrophic associations with methanogens. The proteomic analysis of the partner $D$. desulfuricans confirmed IET via formate and hydrogen carried on by $S$. fumaroxidans in a sulfate rich environment. 


\section{Supporting information}

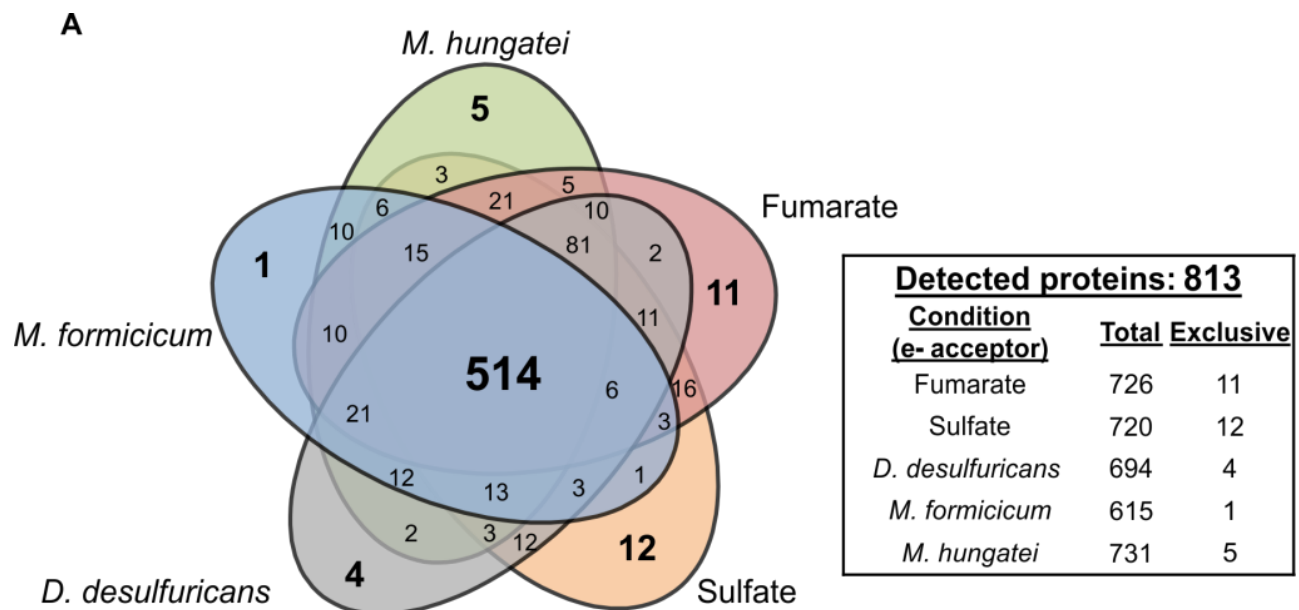

B

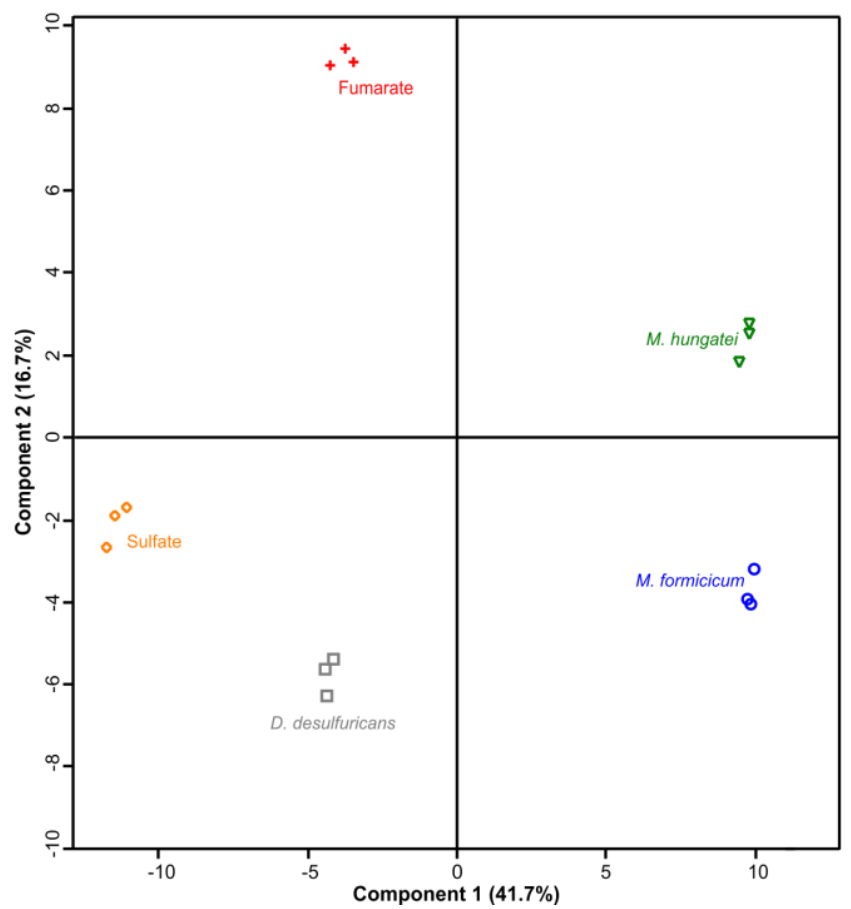

Fig. S4.1. A. Venn diagram of the 813 proteins detected in Syntrophobacter fumaroxidans growth on propionate with five different (biological or chemical) electron acceptors. B. Principal Component Analysis performed for $S$. fumaroxidans protein profiles obtained from each triplicate grown under five different conditions. Symbols: Orange diamonds, sulfate reducing; Red crosses, growth with fumarate; Grey squares, in coculture with Desulfovibrio desulfuricans in a sulfate rich environment; Green triangles, in syntrophy with Methanospirillum hungatei; Blue circles, in syntrophy with Methanobacterium formicicum. 


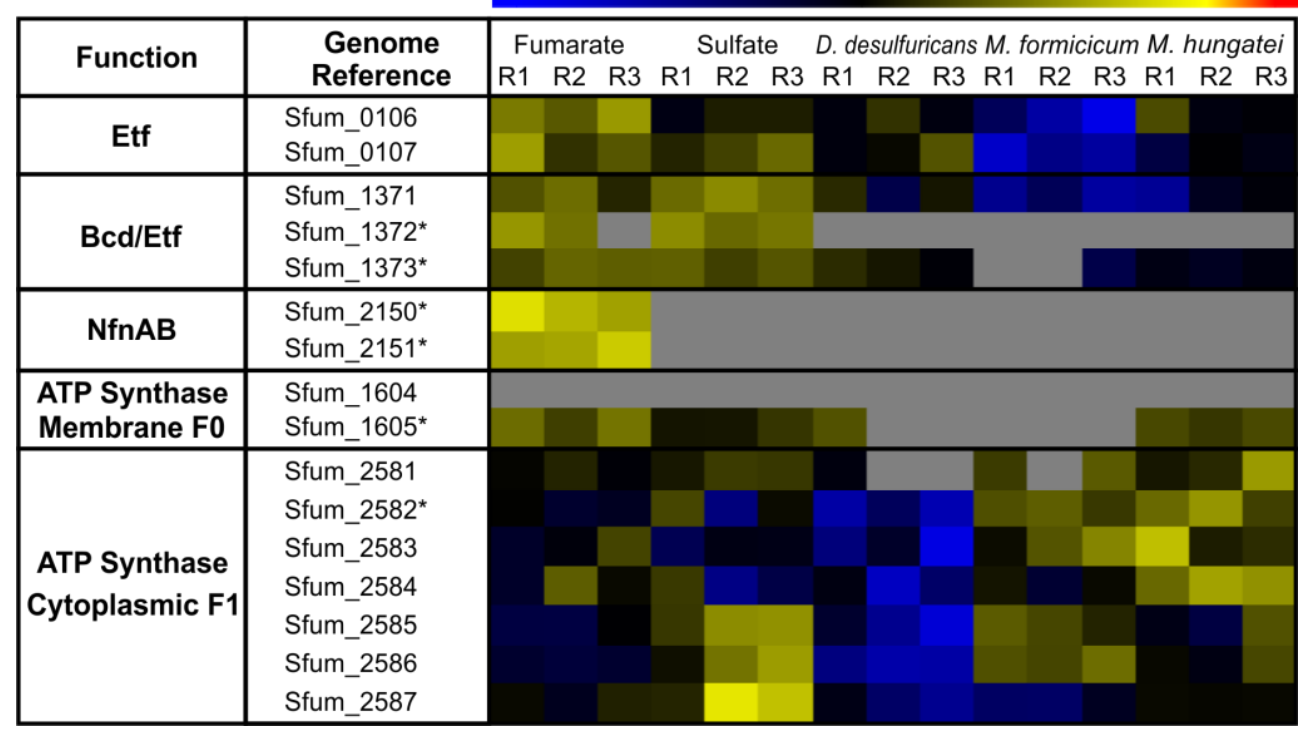

Fig. S4.2. Normalized expression matrix of energy conservation mechanisms predicted for Syntrophobacter fumaroxidans. Proteins are shown for five different growth conditions, in triplicates; from left to right: fumarate, sulfate and interspecies compounds transferred to: Desulfovibrio desulfuricans, $M$. formicicum and $M$. hungatei. The colour scale illustrates the relative detection level of each protein across the 5 samples; blue (log ratio -2.5) and yellow (log ratio 2.5) indicate lower and higher levels compared to the average level value (in black), respectively. Not detected proteins in a specific condition appear in grey. $\left(^{*}\right)$ indicates a statistical significant difference in at least one condition. 

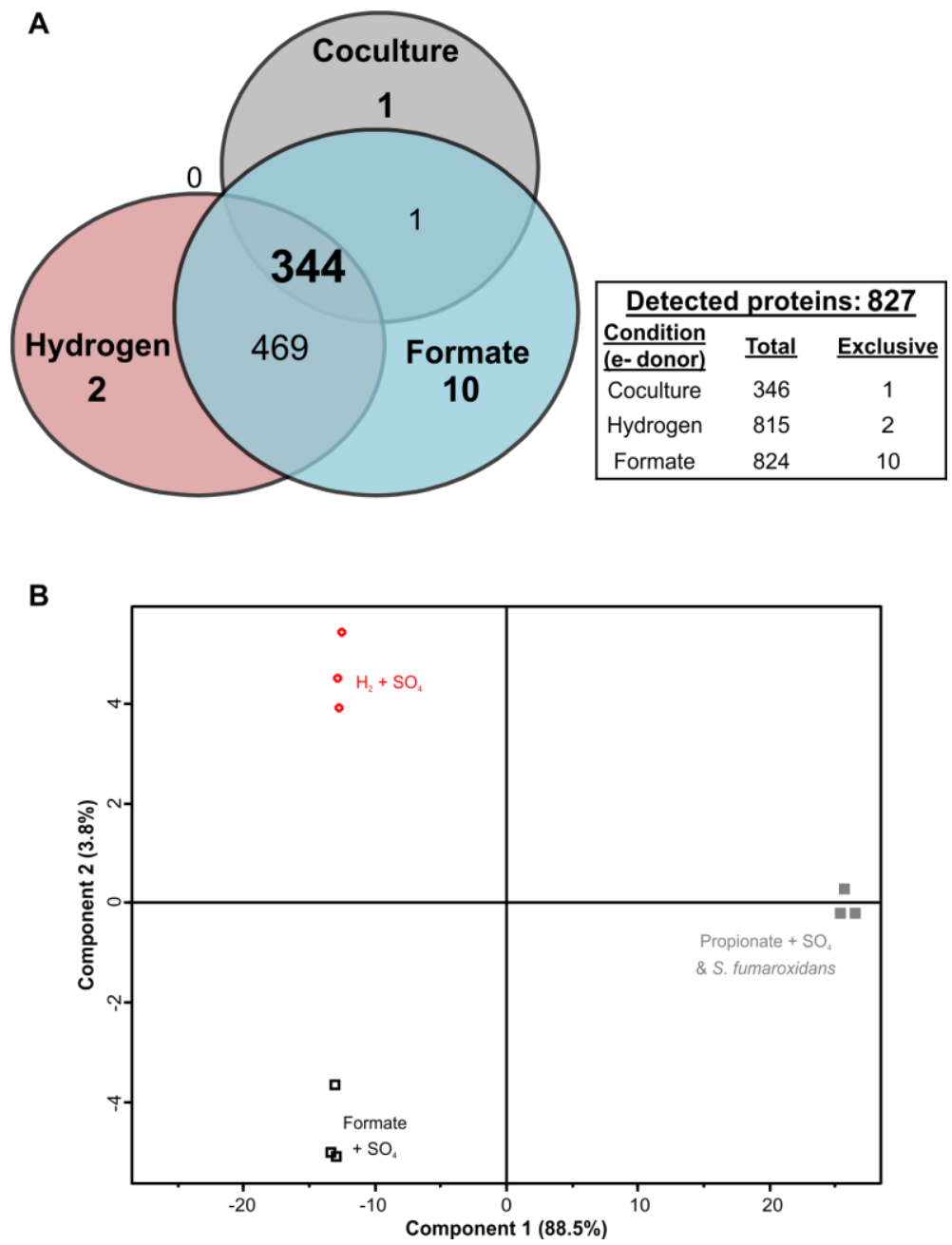

Fig. S4.3. A. Venn diagram of the 827 proteins detected in Desulfovibrio desulfuricans growing in sulfate rich medium in coculture with Syntrophobacter fumaroxidans or axenically on $\mathrm{H}_{2} / \mathrm{CO}_{2}$ or formate. B. PCA performed for $D$. desulfuricans protein profiles. Symbols: red diamonds, hydrogenotrophic conditions; black squares, growth with formate and filled grey squares correspond to the cocultured partnership of $D$. desulfuricans with $S$. fumaroxidans. 


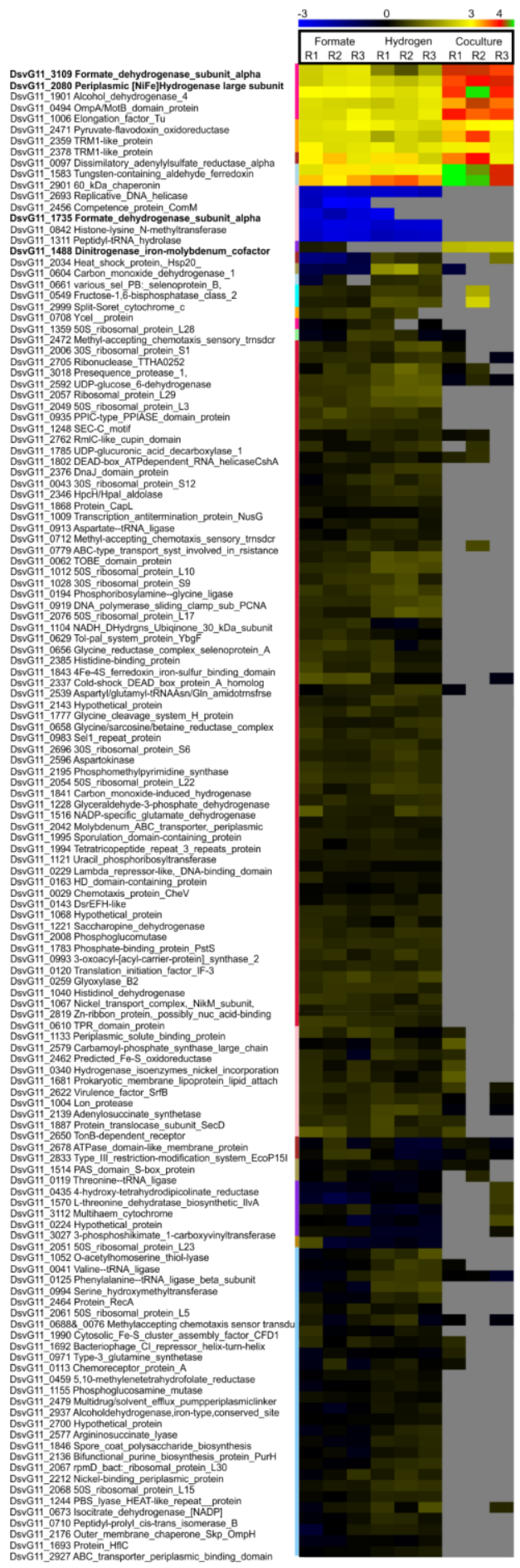

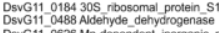

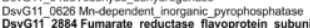

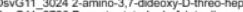

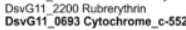

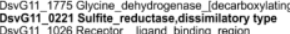

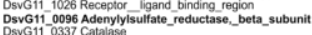

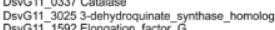

DsvG11-0321 Leucine-speccific-binding protelen

DsvG11 2066 ATP A synthase- subunit

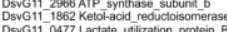

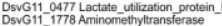

DsvG11-1935 Phosphoenonopyruvate_synthas

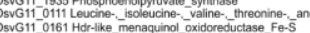

DsvG11-2900 10 kDa chaperonin

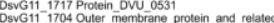

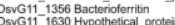

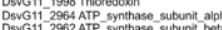

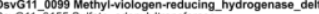

Dsvi11-2098 Chame

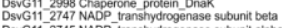

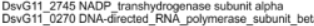

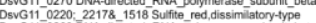

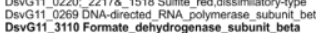

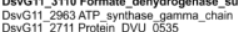

DsvG11-1875 Suffite_reductase. dissimalatory-type_subuni

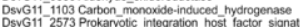

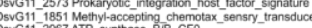

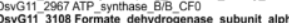

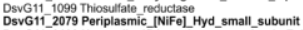

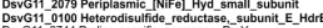

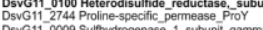

DsvG11-0009 Sulfrydrogenase____subunit__gamma

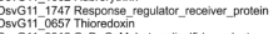

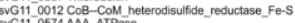

DsvG11-3120 Ferric_uptake_regulator family

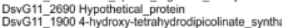

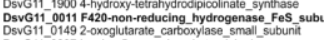

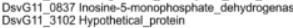

DSVG11-2994 UPF O597_protein_Dde_0807

DSvG11-0115 Dinydroxy-add dêhydratase
DsvG11-1159 Protein translocase subunit SecA

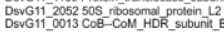

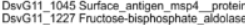

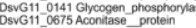

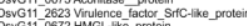

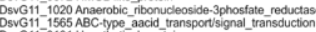

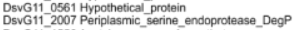

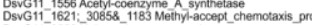

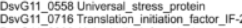

DsvG11-2899 Apha-helical_fereredoxin

DsvG11-2934 Enolase

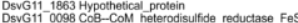

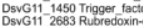

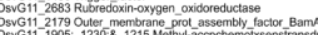

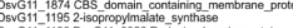

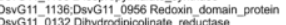

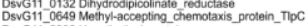

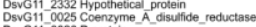

DsvG11-3026 P-proteini

DsvG11-1934 Pyruvate_carboxylase._milochondrial

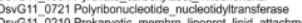

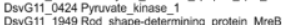

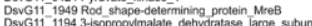

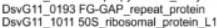

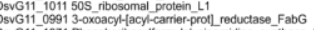

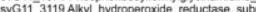

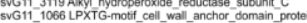

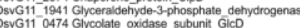

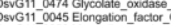

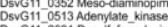

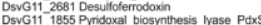

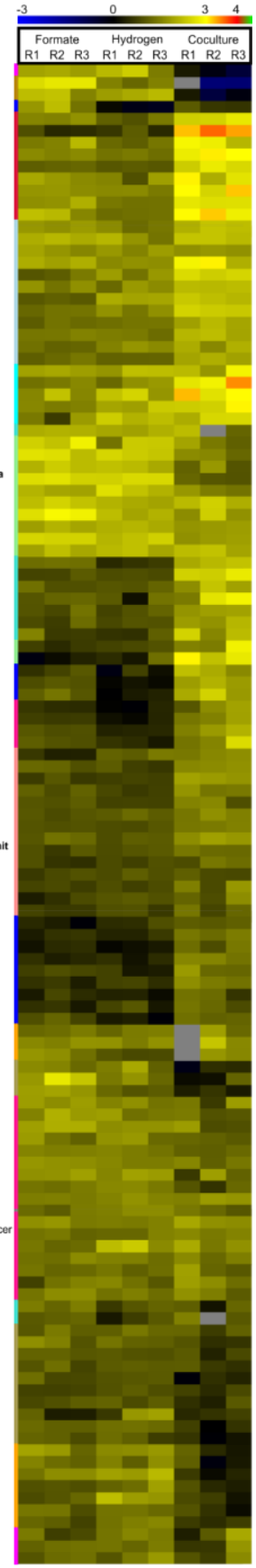


DsvG11-1132 Prin-like_protein_CC_0481
DssG11-1036 LigA

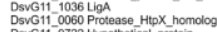

DsvG11-00722 Hypotheticial protein
DsvG11-1468 Nitroreductase

DsvG11-0518 Flavodoxin

SvG11_0511 Acetylomithine aminotransterase

DsvG11-2976 UDP-4-amino-4-deoxy-L-arab DsvG11-2190 UPF0210 proten_Ddes_0622

DsvG11-3042 Phenylacetate-coenzyme_A_ligase
DsvG11-1749 Response regulator receiver_domain_protein

DsvG11-2147 Asparagine-GR RNA ligase
DsvG11-0996 Ribofiavin biosynthesis protein RibD

DsvG11-1711 Glucose-6-phosphate isomerase

DsvG11-1772 Phosphoesterase
DSvG11-2997 Perppasmic binding protein
DsvG11 2380 Tryptophan - synthase_beta_chain

DsvG11_2003 Elongation_factor_P-Particle_protein

DsvG11-0140 Alpha-amylase

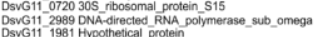

DsvG11-2732 ROSMMUCR__transcriptional_regulator_protein

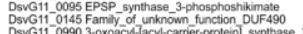

DSvG11-0154 Single-stranded_DNA-binding_ protein _

DsvG11-2950 Dihydroorotate_dehydrogenase _B_NAD+

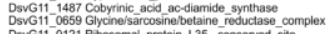

DsvG11 0121 Ribosomal protein L L35.

DsvG11 1613 Flagellar,molor_switch protein FIN

DsvG11-2704 Predicted_Rossmann fold_nucleotide-binding

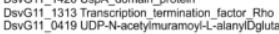

DsvG11-0485 Sulfide dehydrogenase subunit beta
DsvG11-2381 Thymidylate__synthase_ThyX__S__Tase

DsvG11_2159 ATP-binding region_ATPase_domain_protein

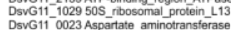

DsvG11-0023 Aspartate aminotranste
DsvG11-0643 Proline-tRNA ligase
DSVG11-2629 2-hydroxy-3-0xopopropion

DsvG11-26292 2-hydroxy-3-3-oxopropionate_reductase

DssG11- 1578 Pancreasiduodenum_home
DSvG11 2227 CheW-like_domain_protein

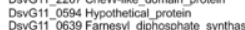

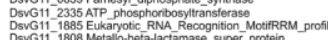

DsvG11-0434 6-phosphoffuctokinase - 6 -
DsvG11- 0540 Malonyl CoA-acyl carrie

DsvG11-1364 Chemotaxis_protein_methyltransterase_2

DSvG11-1041 Fosforibosylaminoimidazol-succino

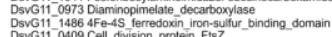

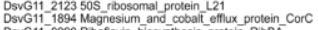

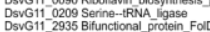

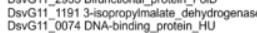

DsvG11_0962 Ferrous iron_transport_protein B
DsvG11_1202 Translation_initiation_factor_fF-1_

DsvG11-1586 Tungstate-ABC, transporter, tungstate-binding

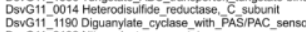

DsvG11-2132 Nitroreductase_protein

DsvG11-0462 Anti-sigma-28_factor, FlgM

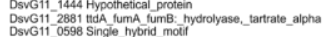

DsvG11_2556 Zinc-transporting ATPase
DsvG11-0152 Biotinflipoyl_attachment_domain-containing

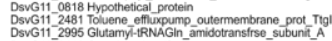

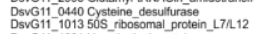

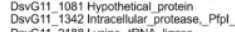

DsGG11-2188 Lysine-1RNA ligase
DsvG11-0788 Yype I I secretion _outermer

membrane_protein Tolc

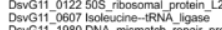

DsvG11 1876 Penicillin-binding protein $1 \mathrm{~A}$-MuS

DsvG11_0151 Acetyl-coenzyme_A_cartoxylase_cartoxyl

DsvG11- 1483 Succinate-semialdehyde_dhy
DsvG11_1975 Triosephosphate__somerase

DSvG11-2239 CTP-synthase -
DsvG11-0917 DNA grase subunit A

DsvG11_0159 Hdr-like_menaquinoi_oxidoreductase_FeS

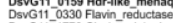

DsvG11-17766 Glycine_dehydrogenase_[decarboxylatingl

DsvG11_ 3111 Protein_involved in formate detydrogena

DsvG11-2606 6-phosphogluconolactonase
DsvG11_0027 Selenocysteine-specific_elongation_factor

DsvG11-2625 PpkA-related_protein
DsvG11_2313 ATPase-like protein

DsvG11_ 1999 Thioredoxin_reductase

DSvG11-0475 liron-sulur ciuster-binding protein

DsvG11-2965 ATP -synthase subunit detta
DsvG11-2600 ABC transporter ATP-binding protein uup

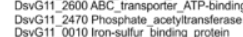

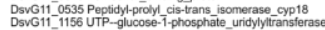

Dsvi11-1156
DsvG11-1000 Lucine-TRNA _ligase

DsvG11- 1000 Leucine-tRNA_ligas
DsvG11_0129 Transketolase -

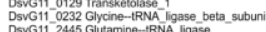

DsvG11-2445 Glutarmine-ARNA ligase
DsvG11 0965 Tryptophan synthase beta_cha

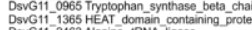

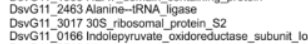

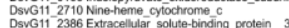

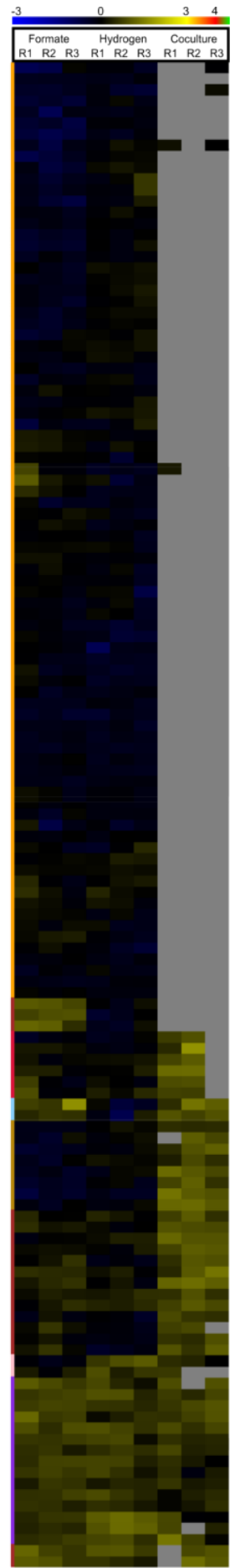

DsvG11_2028 Pantothenate_ synthetase
DsvG11_0936 Glutamine--fructose-6-P_aminotransferase

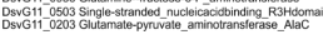

DsvG11 0258 T111655 protein
DsvG11-2055 30 S rbosomal_protein_S3
DsvG11-1703 Protein Toli

DsvG11_0410 Cellilivision_protein_FisA
DsvG11-1105 Hydrogenase-4_component,

DsvG11-0695 NADPH-dependent FMN reductase
DsvG11_2567 RNA_polymerase_sigma_factor_RpoD

DsvG11 0202 Homoserne_dehydrogenase
DsvG11_2148 Aspartate-semalidehyde_detydrogenas

DsvG11-0260 Rhod

DsvG11 09964 Tigptophan__synthase_alpha_chair

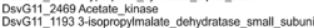

DsvG11-0441 Nitrogen fixation protein_N N

DSvG11_2450 Glyoxylate reductase
DsvG11-0838 GMP - synthase Lglutamine-hydrolyzing]

DsvG11_2056 50S_ribosomal_protein_Lti6

DsvG11-2487 Fosforibosylaminoimidazo

DsvG11_2944 Cytosol_aminopeptidase

DsvG11- 1899 Prokaryotic integration host factor signature

protein-associated_tactor_MO

DsvG11_0147 GTP-bindinnthase

DSvG11_0147 GIP-binding protein_TypABBipA

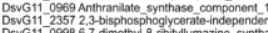

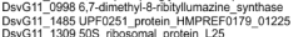

DsvG11_ 1309 50S_rnosomal_protein_L25
DsvG11_2895 D-methionine-binding_lipoprotein_Meto

DsvG11-2027 S-adenosylmethionine_synthase

DsvG11_2064 50S_nbosomal protein L6
DsvG11-2072 30 S-ritosomal protein_S13

DsvG11_2879 Baclerial_extracellular_-solute-binding_protein

DsvG11_0044 30S_ribosomal protein S7
DsvG11_2961 ATP_synthase_F1,_epsilon_subunit

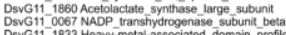

DsvG11-1833 Heavy-metal-associated_domain_profile.
DsvG11_1151 ATP-dependent_zinc metalloportease FFt

DsvG11- 0774 Superoxide dismutase ICu-Zn]

DsvG11-17773 Ribonuclease E

DsvG11-1741 Substrate-binding_region_of_ABC-type_glycin

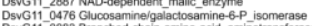

DsvG11_0476 Gluoosamine/galactosamine-6-P isomerase
DsVG11-0083 Branched-chain-amino-acid aminotransteras

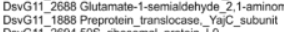

svG11-0687 Prokaryotic integration host_tactor signature

DsvG11_2433 Ribosome-recycyling tacto

DsvG11-1679 Bifunctional purine biosynthesis protein. Purt
DsvG11-0036 Outer membrane chaperone Skp OmpH

DsvG11-0130 Phosphogilycerate-kinaso

DsvG11 2603 RutC p protein_y4sk
DsvG11_0137 Leufleevat-binding protein_homolog_2

G11_ 1289 TRM1-like protein

DsvG11_2907 Adenosylhomocysteinase
DsvG11-1021 Peptidase_M16_inactive domain protein

DsvG11-2035 Flagelar _thament_33_KDa_core_proten

DsvG11-1663 Carboxy-lerminal-processsing _protease

DsvG11-0484 Sulfide, dehydrogenase, subunitalpha
DsvG11-0662 Glycine reductasecomplexoomponent_Bsubunit

DsvG11-1360 Chemotaxis protein_Che

DsvG11_1694 Protein_HAK

DSvG11 2075 DNA-directed RNA polymerase _subunit alph

DsvG11-2048 30 S nibosoma protoin S

DsvG11-1010 50S_ribosomal protein L11

DsvG11_2222 Outer_membrane-a

DsvG11_2578 Argininosuccinate_-synthase
DsvG11_2074 30S_nibosomal_protein_S4

DsvG11_2021 Dissimiatlory sulfite réductase_D_DsrD

perplasmic binding prote

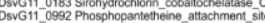

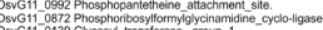

DsvG11-2691 Eukaryotic RNA Recognition_Moti__RRMprofile

DsvG11_ 1197 Phosphatitcylserine _ecarboxylase proenzyme

DsvG11-1886 Protein transioce _.FA_tinding

DsvG11-1303 Cysteine-tRNA_ligase
DsvG11_1102 4Fe-4S_ferredoxin_iron-sultur_binding_domain DsvG11-0539 Argininie-ARNA ligase
DsvG11 0908 Stage 0 sporviation protein

DsvG11 0547 4hydroxybenzoate_decarboxylase_subunit C C
DsvG11-0158 Hdr-like menaquinol oxidoreductase Cytochr

DsvG11_ 1739 Glycine betaine_transport_ATP-binding prote

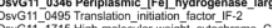

DsvG11_-2030 Signaling protein Ykow

DsvG11-1419 UITT6 protein

DsvG11_0867 Mitochondrial__small ribosomal subunit_Rsm22

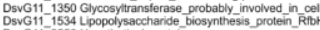

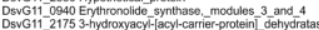

DsvG11-21753-hydroxyacy-lacyl-carrier-protein[- dehydratas

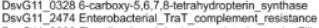

DsvG11-3038 Flagellar basa'_body-associated_protein

DsvG11 2850 Tolo protein

DsvG11-2661 Mutitidug resistance_protoin_Mate

OssG11-2401 Hypothetical protein
DsvG11 2125 ATP synthase_protein

DSvG11-1764 Hydrogenase_expressioniflormation_Hype

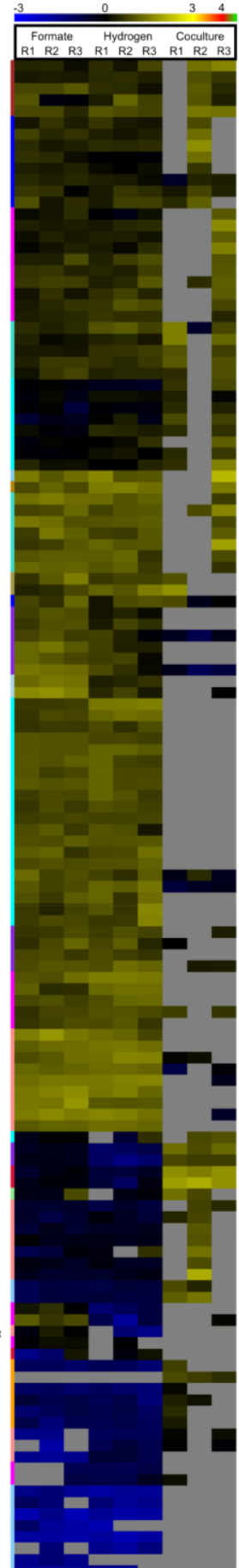


DsvG11_0342 TPR_repeat

DsvG11-0190 Cupin domain

DsvG11_2561 GGDEF_domain-containing protein

DsvG11-2268 Cytochrome_C_Diogenessis transmembrane
DSVG11-_1924 Glutamate/Asp_transport_ATP-binding_protein

DsvG11-1697 Ribonuclease -

DsvG1_-0517 Radical_SAM_domain_protein

DsvG11- 1856 Hypothetical_protein
DssG11-2535 Zinc_ribbon domain

DSVG11-2690 GTP Pyrophosphokinase
DsvG11-2730 Adenosymmethionine-8-ar
DsvG

-7-oxonoNaNoate

DsvG11- 1094 NADH-quinone_oxidoreductase_subunit__D_1

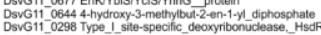

DsvG11-0556 Cydidylate_kinas -

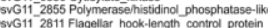

DsvG11-2645 2,3-ihindroxybenzoate-AMP _ligase

DsVG11-2538 Protein of unknown_function_DUF89

DsvG11-1453 Lon protease
DSvGG11-1083 Hypotheitcal prote

DsvG11_0982 Molecular_chaperone-/ike_protein
DsvG11-1059 Signal_recognition particle_receptor_Fits Y

DSvG1_-1059 Signal_recognition
DsvG11-2161 1PR_repeat_trote

DsvG11- 1750 Histidine kinase

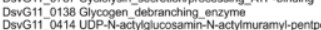

DsvG11-2595 Hydrolase, Ploop

DsvG11-0491 Precomin-6Y C5, 15-mettyytransferase

DSvG11-1224 Thymidylate-kinase

DsvG11-1539 Glycosyl_transterases group_-
DsvG11_2689 Transcriptional_regulators

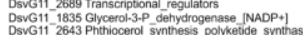

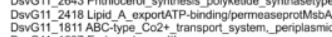

DsvG11 0858 Polysialic acid_transport protein _Kos

SsVG11-1446 DNA__ligas

DSVG11-1845 D - ant LIC L 12162.putativetransterase, LIC12162

DsvG11-0942 Capsule polysaccharide_export_protein_BexD

DssG11-2106 Hypothetical protein
DssG11-1916 Flagellar assermbly protein_Fit

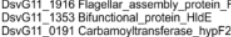

DsvG11-0207 L-serine _tehydratase_-hyp

DSvG11-2624 Tetratricopeptide-like helical
DSvG11-2000 Outer_membrane protassembly_tactor_BamD

DsvG11-0887 OLD - ATP-dependent endonudiease
DsvG11-2684 Class III. cylochrome C C signature

DsvG11-1607 Cylochromeec c class
DsvG11- 1171 OsmC-like protein

DsvG11-1174 Hypothetical_protein
DsvG11
De250 Putative exonuclease, Rdgc

DsvG11_0250 Putative_exonuclease, RdgC

DsvG11-0885 Typpe__ sinte-specific_donxynbonuclease,_HisdR

Dsv11-1714 Hypotheticial protet
DsvG11_1357 Rubredoxin-2

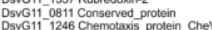

DsvG11-2238 Phosphoribosyltormyglycinamididine_synthase

rane_lipoprōtein carrier_prot_LolA

DsvG11-1872 Hypothetical_protein

DsvG11-2815 Stage III sporilation protein
DSvG11-0565 Transcriptonal reguatory protein Z ZraP

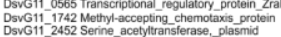

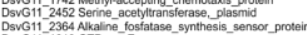

DsvG11-1610 GTPase_Era

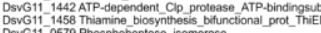

DssG11 2743 Alanine racemase
DsvG11-261917-kDa_surface_antigen
DssG11-1297 Redoxin domain protein

DSvG11-1297 Redoxin _domain - protein
DSvG

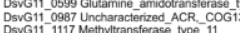

DsvG11-1117 Methytransferase_type-11

DsvG11-1118 TonB-dependent-1119 Formyimethan
DsG11
DsvG11-0320 GTPase_Der

DsvG11-2980 L-actate - permease
DsvG11-0989 Phosphate acyltransteras

DsvG11-1451 ATP-dependent CIP_protease_proteolytic_sub

DsvG11-3011 Tyrosine-tRNA_ligase
DsvG11-2122 50 _ribosomal-protein_L27

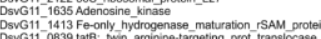

DSvG11-0839 tait: Hin arginina-targeting prot transiocase

DsvG11_0186 Prokaryotic_membrane__lipoporotein__ipid__atac

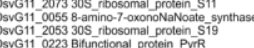

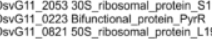

DsvG11-0841 Imidazoleglycerol-phosphate_dehydratase

DsvG11- 1326 Hypothetical protelin
DsvG11_1164 Benzoyitormate decarboxylase

DsvGG1-10886 DNA helicase II
DsvG11-2530 Transporter SSS

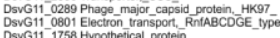

DsvG11-1758 Hypothetical protien
DssG11-1482 Dihydroorotase

DsvG11-0918 DNA _oyrase_subunit_B
DsvG11-0860 RmiC-like_cupin_domain

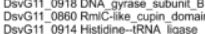

DsvG11-0699 3methyl20xobutanoate hydroxymethyltransferas

DsvG11_2466 RNA_polymerase-binding protein_DKsA

DsvG11-1748 Hypothetical protein

DsvG11-2912 Aipha-helical_ferredoxin
DsGG11-1560 Copper-expotring Ptype_ATPase_A

DsvG11-0665 Vi_ polysacoc_biosynthesis protein_VipB/TviC

DsvG11-2984 Thiazole_symithase

DsvG11-1523 M18_aminopeptidase
DsvG11_2943 Porphyrin_biosynthesis_protein_HemD

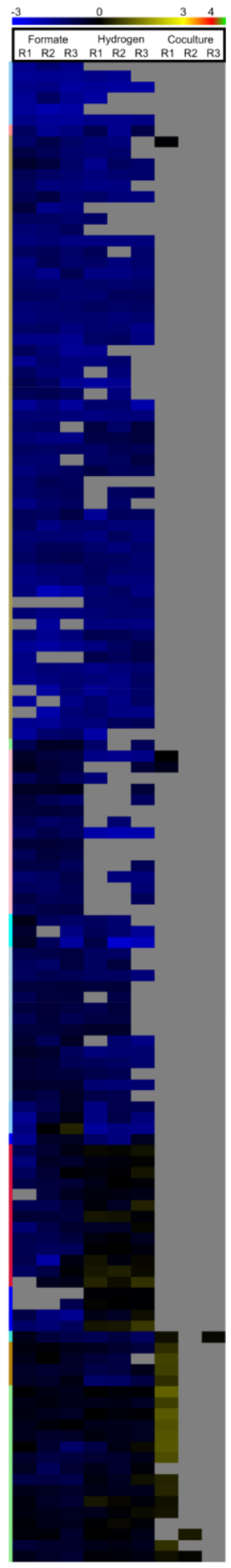

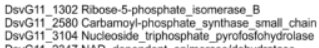

DsvG11-2347 NAD dependent-epimerase/dehydratase

DsvG11-0878 UDP-N-acetyiglucosamine

omain_protein

DsvG11 2398 UvrABC_system_protein_A

DsvG11-1688 D-alanine-D-dalanine_ligase
DSvG11_1760 Solutile__ydrogenase, _small_subun

DsvG11_-1160 Molybdopterin_biosynthesis MoaE_protein

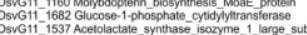

DSWG11-2620 Trpsin
DsvG11-1870 Al--rans-nonaprenyl-diphosphate synthas

DsvG11_0915 Amidophosphoribosyltransferase_2, chlorople

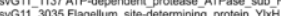

DswG11_2957 Elongation_factor_4

DsvG11_2761 Potassium_ethux_system_KerA

DsvG11_1671 Putative_zinc_or iron-chelating_domain

DsvG11-2015 DNA repair protein RecN
DsvG11-2422 Flagellar hook-associated 2 domain protein

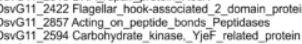

Divi1-

nal_regulatory_protein_FIt

DswG11-0580 ADPP-L-glycero-D-mannoup - 1 D protein

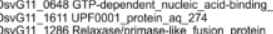

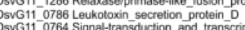

osfate_synthase_subunit DsuG11-0445 Phosphogycolate phosphatase

tion tactor 6 . 2

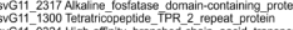

Dsti-12265 Huch-afinity branch

D.

11__1557 Hypothestical_protein

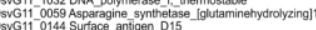

DsvG11-2240 RNA polymerase_sigma-54 factor

SvG11_0533 Ribonuclease R R -

DsvG11-1168 Hypothetical protein
DsvG11-2320 Nudlease SbcCD subunit_C

DsvG11 2473 Methyl-accepting chmbxis_sensory_transducer

DsvG11- 1873 UPF0272 protein_Moth_2512

Dsv611-1287 Hypothetical_Lrotin
DsvG11-0005 Hypothetical protein

DsvG11 0840 Sec-independent prot translocase _prot TatC

DsvG11 0216 Peptide chain release factor_ 1

DsvG11-0300 Super I DNA and RNA helicases_\&_ helicase

regulator prot-gutamate

DSV11- 1295 MJ0042 - finger-like,proten

SVG11 0548 Glutamine-dependent NAD+-synthetase

DsvG11_0343 AMIN_doma

DsvG11-2520 Hypothetical protein

DsvG11-0975 Amidohydrolase
DsvG11-1832 Copper-exporting_P-type_ATPase_A

DsvG11-1832 Copper-exporting

DsvG11 0843 1.5-phosphoribosyl-5-5-10storibosylaminom

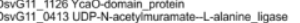

DsvG11-2835 Type III restriction-modification_system_EcoP

DsvG11 2938 L-glutamine:2-deoxy-scyllo-inosose

111-1670 4Fe-4S_Fd-type iron-s

DSvG11 1069 Ferrous iron_transport_protein_
DsvG11_2534 Porphobolinogen_deaminase

DsvG11-0700 Agmatinase 1

DsvG11_2759 Thiamine-phosphate_synthase

DsvG11-2232 Hypothetical protein

ssvG11-18394 4Fe-4S_binding_domain_protein

svG11_0325 High-affinity branched-chainaminoacidtransp

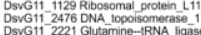

DsvG11- 1996 Fructose-1,6-bisphosphatase_class_-

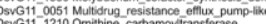

DsvG11-2933 Type- III-_pantothenaterantekinase

boxylase

ridoreductase

DsvG11_2355 Phenylacetate-coenzyme_A_ligase

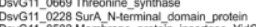

DsvG11_0679 Adenylosuccinate ily

DsvG11_2641 Bacitracin_synthase 1 .

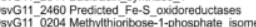

SVII1_1542 Radical_SAM

svG11-0418 UDPNacetyimuramoyitripeptide-D-alany-D-alan

DsvG11-3107 Formylmethanoturan dehydrogenase, sub_

DsvG11_0763 Sulfoacetaldehyde acelytransferase

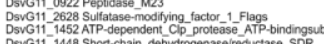

DsvG11-1448 Shor-chain_de hydrogronaseredreductase_SDR

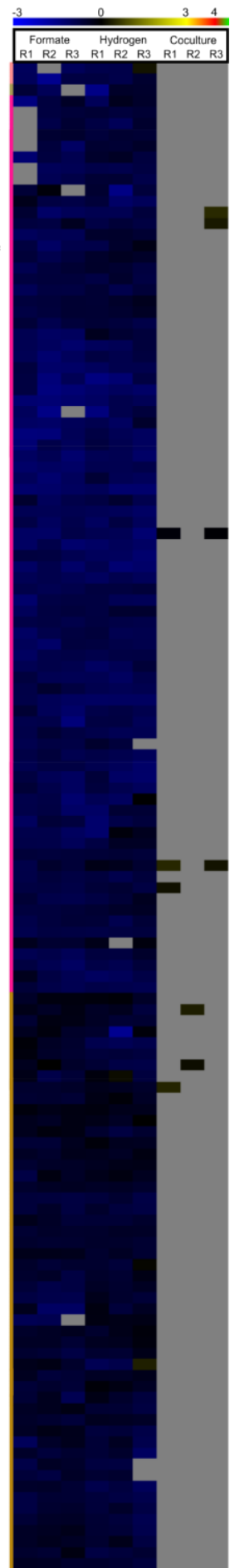




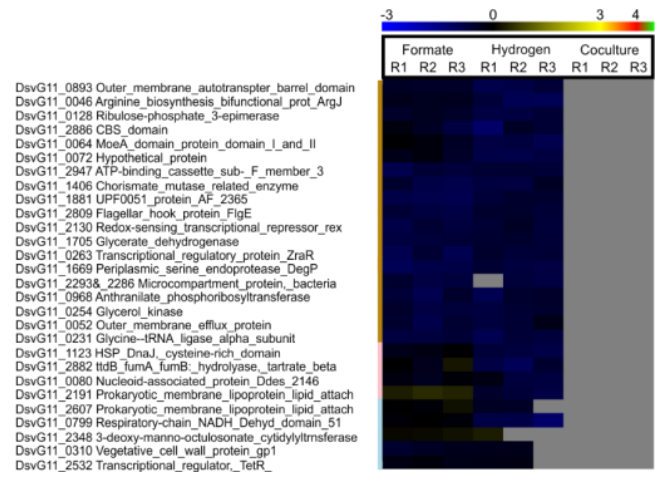

Fig. S4.4. Heat map of hierarchical clustered proteins produced by Desulfovibrio desulfuricans. The proteins are shown in a clustered matrix after column Z-score normalization and automatic hierarchical columns clustering. Three growth conditions, in triplicates, are shown according to the electron donor used; from left to right: formate, hydrogen and compounds transferred from Syntrophobacter fumaroxidans. The colour scale represents the relative detection level of each protein across the samples; blue log ratio -3, yellow log ratio 3, red log ratio 4 and green log ratio 4.5 indicate lower and higher levels compared to the average level value in black, respectively. The colour intensity indicates the degree of protein up- or down regulation; the grey colour represents not detected. 


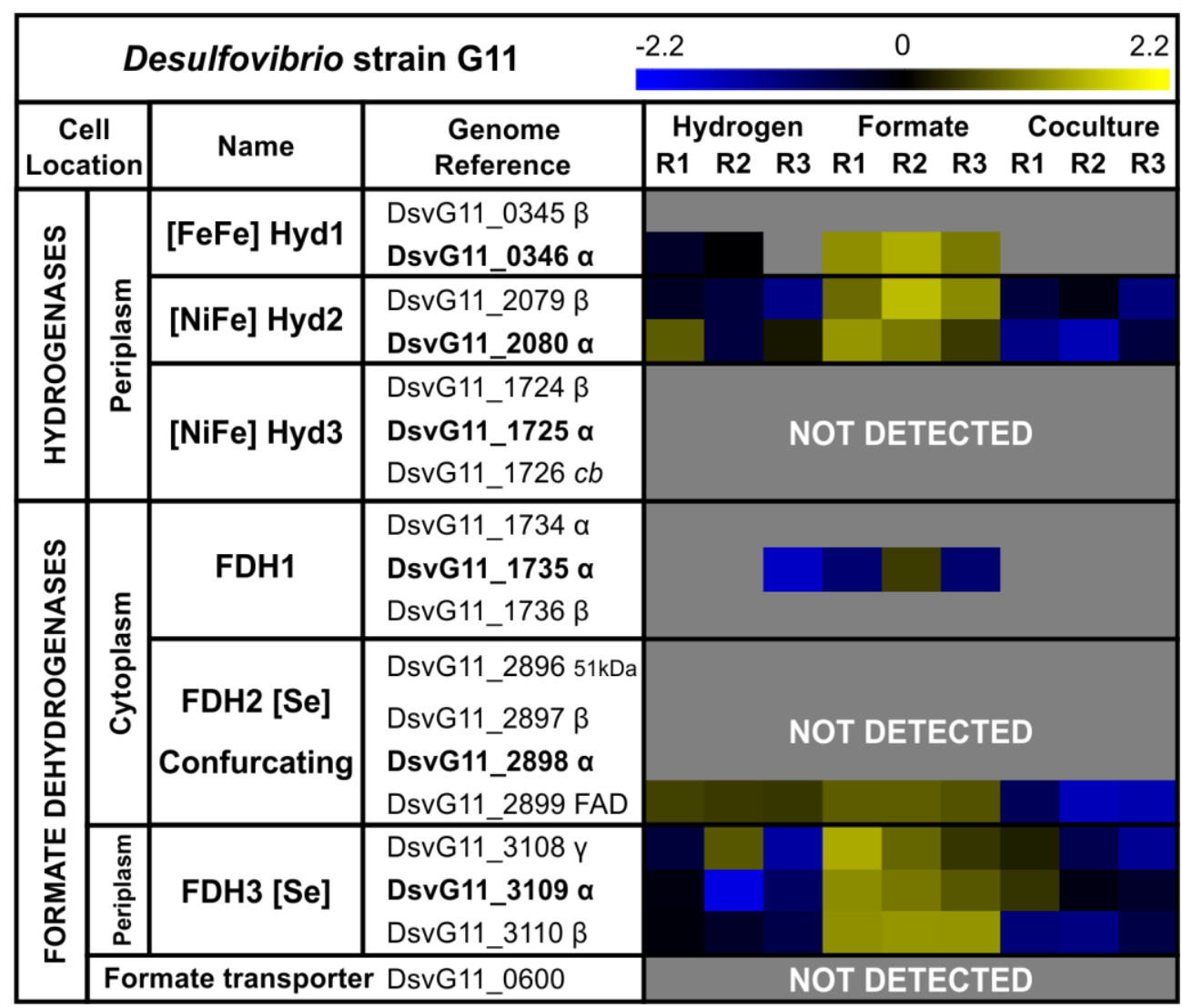

Fig. S4.5. Normalized expression matrix of hydrogenases and formate dehydrogenases of Desulfovibrio desulfuricans. The rows in the heat map show proteins levels after row Z-score standardization in three different growth conditions. The columns show from left to right, in triplicates, the electron donor used by $D$. desulfuricans: hydrogen, formate and interspecies compounds transferred from Syntrophobacter fumaroxidans. The colour scale indicates the degree of protein down- or up regulation ranging from blue (-2.2 log ratio), to yellow (2.2 log ratio). The colour intensities indicate lower and higher levels compared to the average level 0 value (in black); the grey colour represents not detected. Subunits are indicated after the locus tag. 


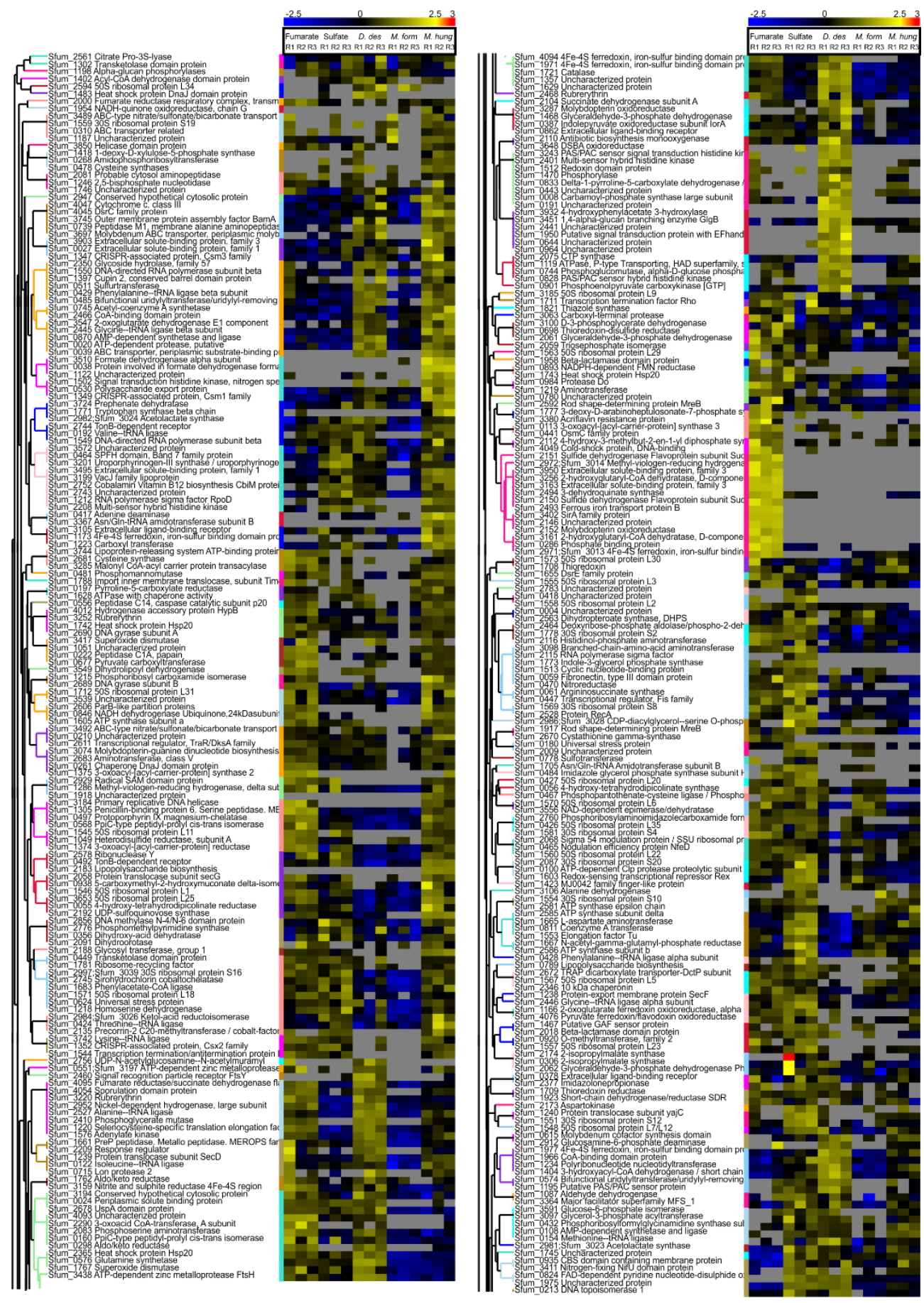



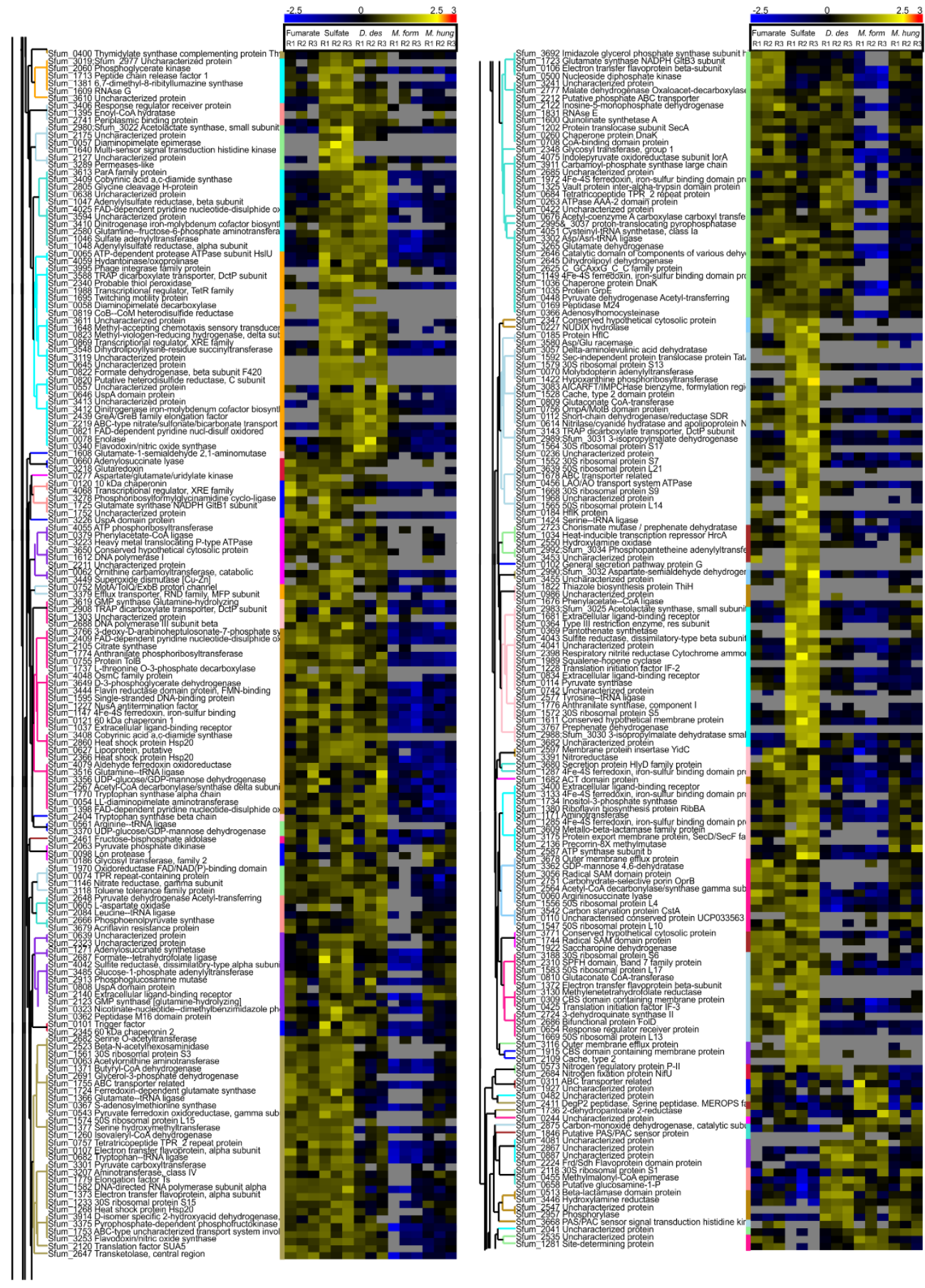

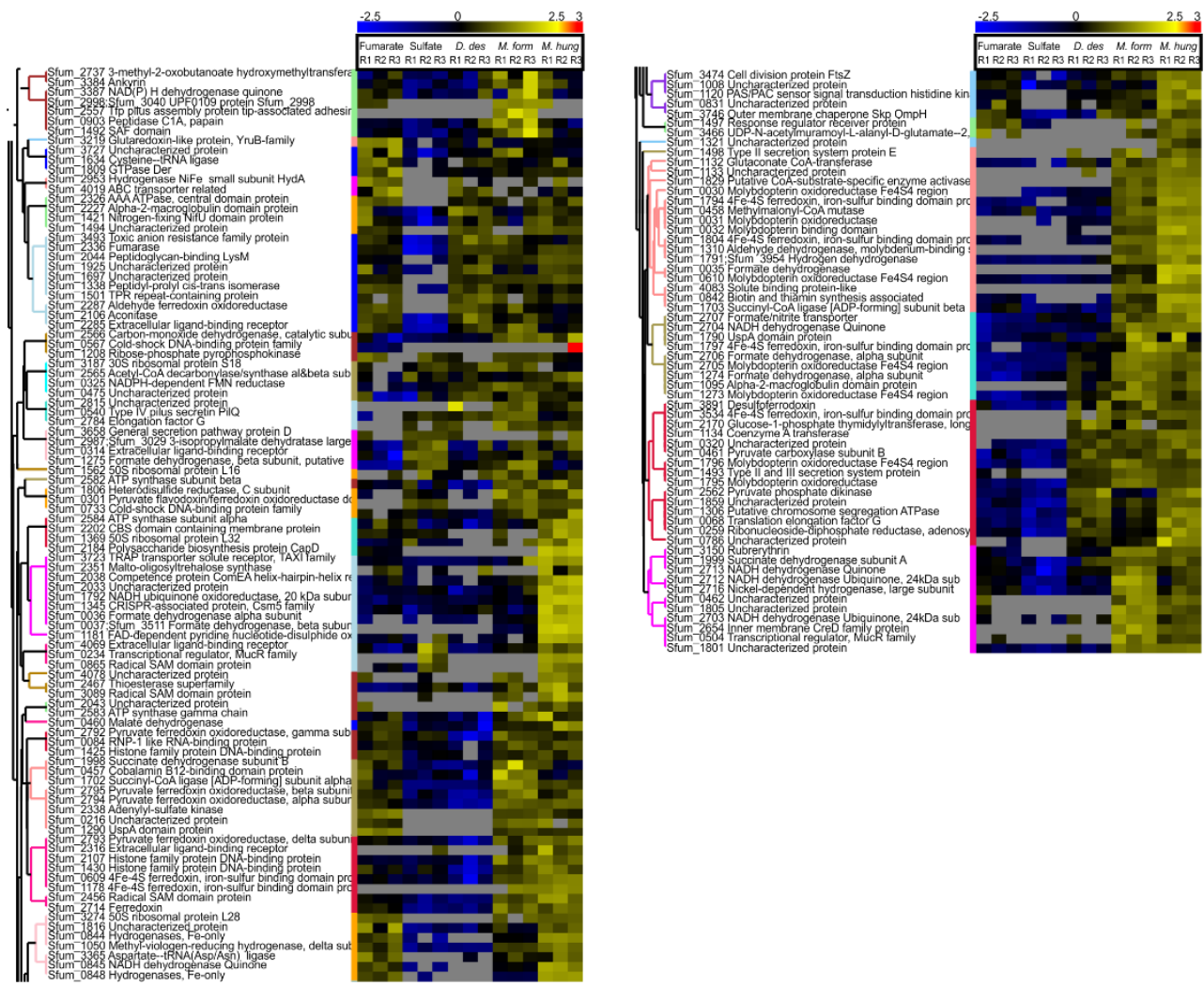

Fig. S4.6. Heat map of hierarchical clustered proteins produced by Syntrophobacter fumaroxidans for propionate degradation. The proteins are shown in a clustered matrix after automatic hierarchical cluster of rows from row Z-score normalization values. Proteins appear from left to right, in triplicates, according to the growth conditions defined by the electron acceptor used by $S$. fumaroxidans to oxidize propionate: fumarate, sulfate and interspecies compounds transferred to: Desulfovibrio desulfuricans, Methanobacterium formicicum and Methanospirillum hungatei. The colour scale illustrates the relative detection level of each protein across the samples; blue log ratio -2.5 , yellow log ratio 2.5 and red log ratio 3 indicate lower and higher levels compared to the average level value 0 in black. The colour intensity indicates the degree of protein up- or down regulation; the grey colour represents not detected.

Fig. S4.7 Schematic representation of energy converting complexes and proteins involved in sulfate reduction in Syntrophobacter fumaroxidans during propionate oxidation. 


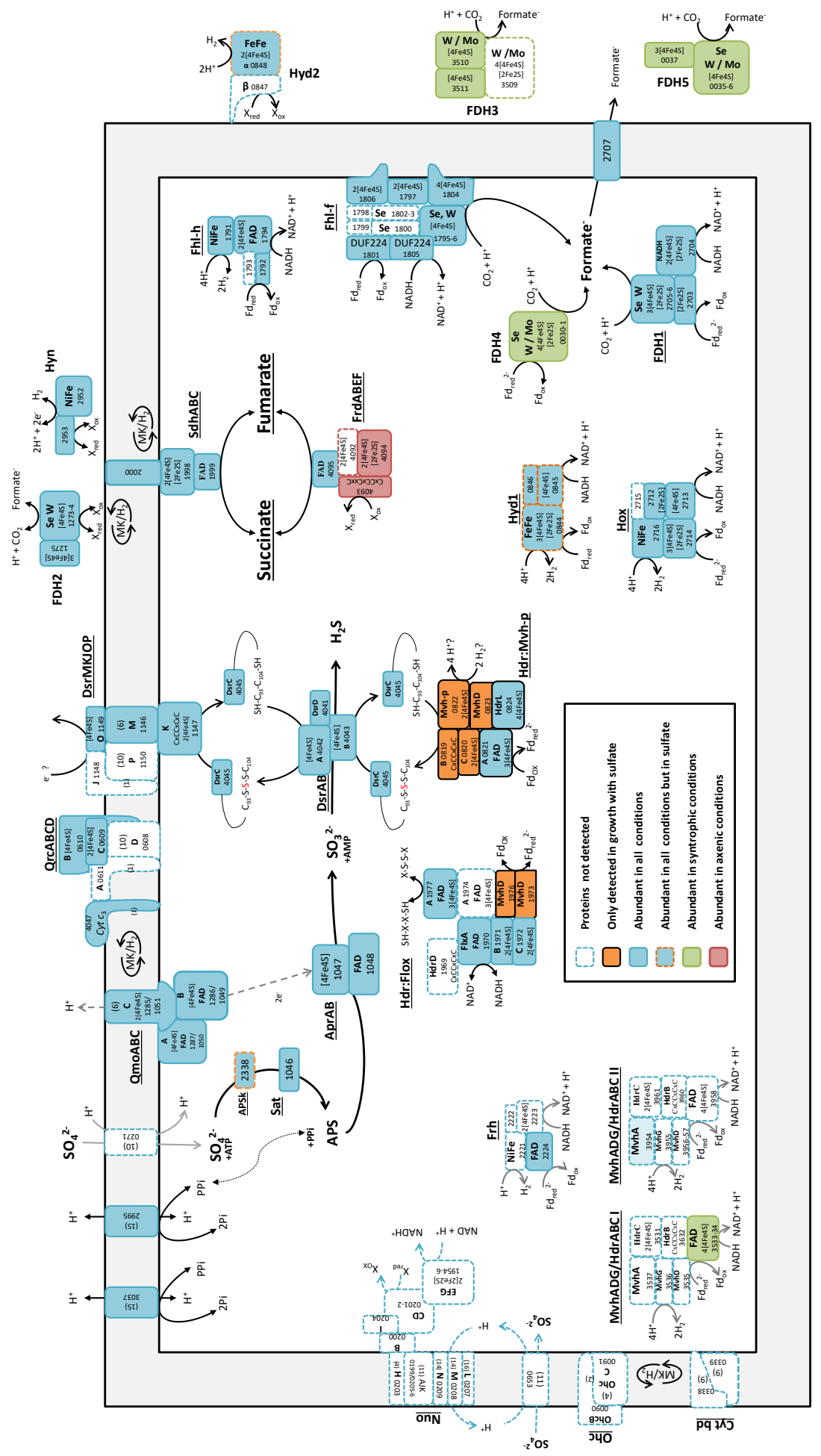


One need not make it first... but one must know how to make it! 
ใ

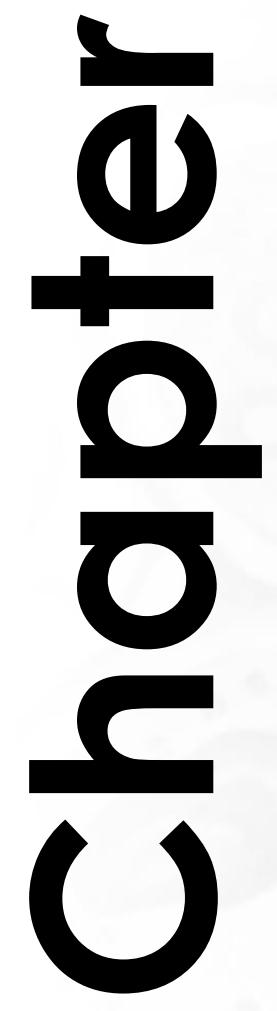




\section{CHAPTER 5}

\section{Proteomic analyses of Methanospirillum hungatei and Methanobacterium formicicum grown in a syntrophic partnership with Syntrophobacter fumaroxidans and in pure culture with $\mathrm{H}_{2} / \mathrm{CO}_{2}$ or formate.}

\footnotetext{
Vicente T. Sedano-Núñez ${ }^{1}$, Sjef Boeren ${ }^{2}$, Caroline M. Plugge ${ }^{1}$ and Alfons J. M. Stams ${ }^{1}$

${ }^{1}$ Laboratory of Microbiology, Wageningen University and Research, The Netherlands

2Laboratory of Biochemistry, Wageningen University and Research, The Netherlands
} 


\begin{abstract}
The hydrogenotrophic methanogens Methanospirillum hungatei and Methanobacterium formicicum are frequently used as syntrophic partners in methanogenic cocultures. We performed a proteomic analysis of these methanogens grown on $\mathrm{H}_{2} / \mathrm{CO}_{2}$, formate and in syntrophy with the propionate-degrading bacterium Syntrophobacter fumaroxidans. We identified the most abundant proteins used for methane formation and energy conservation, and discussed differences among the cultured conditions and between the methanogens. $M$. formicicum uses a $\mathrm{F}_{420}$-non-reducing hydrogenase (MvhADG) for bifurcation to couple the final methane-producing step catalysed by heterodisulfide reductase ( $\mathrm{Hdr}$ ), to the initial $\mathrm{CO}_{2}$-reducing step catalysed by formylmethanofuran dehydrogenase (Fmd). $M$. hungatei lacks the MvhAG subunits of the $\mathrm{F}_{420}$-non-reducing hydrogenase MvhADG, instead it employs an $\mathrm{F}_{420}$-reducing hydrogenase (FrhADGB). Moreover, $\mathrm{F}_{420}$ dependent formate dehydrogenases are also used by both methanogens, predominantly in syntrophic growth, for bifurcation. Differential production of enzymes such as Mcr, Mrt and Hdr were found in the methanogenic pathway as well as in diverse extracellular structures such as archaellum and pili. Although both methanogens can grow on hydrogen and formate, the molecular mechanisms analysed in this study, points to the use of hydrogen in $M$. formicicum, and of formate in $M$. hungatei, as electron carriers in their metabolism.
\end{abstract}

Keywords: Methanogenesis, hydrogen, formate, electron transfer, electron bifurcation, syntrophy, Methanomicrobiales, Methanobacteriales. 


\section{Introduction}

Hydrogenotrophic methanogens grow by reducing $\mathrm{CO}_{2}$ with hydrogen to methane. Many hydrogenotrophic methanogens are also able to use formate for growth (Thauer et al., 2008). Methanogens play an essential role in degradation of volatile fatty acids by removing the excess of hydrogen produced by acetogenic bacteria. In the absence of inorganic electron acceptors, the overall anaerobic degradation of fatty acids becomes energetically feasible only at low hydrogen and formate concentrations (Schink, 1997; McInerney et al., 2008; McInerney et al., 2009; Stams and Plugge, 2009). The capacity of the methanogens to keep concentrations of hydrogen and formate very low has been used to obtain syntrophic cocultures with anaerobic acetogenic bacteria degrading butyrate (McInerney et al., 1979), propionate (Boone and Bryant, 1980) and acetate (Zinder and Koch, 1984) among other short and long chain fatty acids (Sousa et al., 2007). Since these methanogenic partners can use both hydrogen and formate, the role of each in interspecies electron transfer (IET) is not clear. The importance of formate as electron carrier in IET was addressed before (Boone et al., 1989), particularly in the syntrophic degradation of propionate (Dong et al., 1994; Dong and Stams, 1995; de Bok et al., 2002b).

In this study, we investigated the metabolism of two hydrogenotrophic methanogens growing in pure culture with formate or $\mathrm{H}_{2} / \mathrm{CO}_{2}$. Furthermore, we compared the axenic growth of these methanogens to their growth as syntrophic partners of Syntrophobacter fumaroxidans strain $\mathrm{MPOB}^{\mathrm{T}}$, a propionate-degrading bacterium. Methanospirillum hungatei strain $\mathrm{JF}^{\mathrm{T}}$ is a formate- and hydrogen-utilizing methanogen (Ferry and Wolfe, 1976) and the model partner of S. fumaroxidans (Stams et al., 1993). The draft genome of $M$. hungatei has been analysed (Worm et al., 2011b), and its complete genome sequence was described (Gunsalus et al., 2016). Methanobacterium formicicum strain $\mathrm{MF}^{\mathrm{T}}$ is another hydrogen- and formateutilizing methanogen (Bryant and Boone, 1987) which also has been used in syntrophic studies with S. fumaroxidans (Dong et al., 1994; Worm et al., 2011b) and to obtain defined syntrophic associations with Pelotomaculum schinkii (de Bok et al., 2005) and Syntrophomonas zehnderi (Sousa et al., 2007). Although the genome of the neotype strain Methanobacterium formicicum $\mathrm{MF}^{\mathrm{T}}$ and of a strain of rumen origin M. formicicum BRM9 are available, their analysis is limited (Kelly et al., 2014; Maus et al., 2014).

Here, we extended the genomic analysis of $M$. formicicum and with a proteomic analysis of both methanogens we studied and compared their metabolism in the three culture conditions. Our aim was to pinpoint the key enzymes that vary between the two electron donors, $\mathrm{H}_{2}$ and formate, and between axenic and syntrophic growth, as well as to understand the differences between the two methanogens. 


\section{Materials and methods}

\section{Organisms and growth conditions}

Cocultures of Syntrophobacter fumaroxidans MPOB $^{T}$ (DSM 10017) with Methanospirillum hungatei $\mathrm{JF} 1^{\mathrm{T}}$ (DSM 864) or with Methanobacterium formicicum MF $^{\mathrm{T}}$ (DSM 1535) were grown with $30 \mathrm{mM}$ of propionate without electron acceptor in anaerobic medium as described previously (Stams et al., 1993). Axenic cultures of $M$. hungatei and $M$. formicicum were grown with $40 \mathrm{mM}$ formate or with hydrogen (1.7 atm $\mathrm{H}_{2} / \mathrm{CO}_{2}$ 80:20 vol/vol) and supplemented with $1 \mathrm{mM}$ of acetate. All microorganisms were batch cultured in triplicate at $37^{\circ} \mathrm{C}$ in 1-litre flasks with 550 $\mathrm{ml}$ medium under anaerobic conditions provided by a gas phase of $172 \mathrm{kPa}$ (1.7 atm) $\mathrm{N}_{2} / \mathrm{CO}_{2}$, or $\mathrm{H}_{2} / \mathrm{CO}_{2}(80: 20$, vol/vol) when hydrogen was required. Cells were harvested during mid-exponential growth phase. The cultures for the experiment were inoculated with cells from cultures that were transferred at least ten times on their respective electron donor before the start of the experiment.

\section{Harvesting cells and Percoll gradient centrifugation}

Cells were aerobically harvested by centrifugation at $16,000 \mathrm{~g}$ for 16 minutes at $4{ }^{\circ} \mathrm{C}$. The pellet was washed twice with TE buffer (10 mM Tris-HCl, pH 7.5; 1 mM EDTA). Only cells from the syntrophic coculture of $S$. fumaroxidans and $M$. hungatei were separated by Percoll gradient centrifugation (Percoll ${ }^{\circledR}$, Sigma-Aldrich, MO) as described elsewhere (de Bok et al., 2002a). The separated layers, containing Syntrophobacter cells in the upper layer and Methanospirillum cells in the lower layer, were collected and subjected to Percoll gradient separation a second time. Cells were then washed twice with $10 \mathrm{mM}$ sodium phosphate buffer ( $\mathrm{pH}$ 7.5).

\section{Protein extraction and SDS-PAGE}

Cells were resuspended in lysis buffer (100 mM Tris-HCl, $\mathrm{pH} 7.5 ; 4 \%$ w/v SDS; 50 $\mathrm{mM}$ dithiothreitol and SIGMAFAST ${ }^{\mathrm{TM}}$ Protease Inhibitor Cocktail Tablet (SigmaAldrich, MO)), and passed three times through a French press (French ${ }^{\circledR}$ Type Pressure Cell Disrupter, Stansted Fluid Power, Harlow, UK) at $2 \mathrm{MPa}$ (40K cell). Cell debris and undisrupted cells were removed by centrifugation at 18,000 g for 10 min at $4{ }^{\circ} \mathrm{C}$. The supernatant was collected in Eppendorf ${ }^{\mathrm{TM}}$ LoBind Protein Microcentrifuge Tubes and stored at $-80^{\circ} \mathrm{C}$. Still in the lysis buffer, proteins were denatured by heating at $95{ }^{\circ} \mathrm{C}$ for 5 minutes. Samples were loaded on a $10 \%$ polyacrylamide separation gel (25201, Precise ${ }^{\mathrm{TM}}$ Tris-HEPES Gels, Thermo Scientific, Rockford, US) using the Mini-PROTEAN Tetra Cell (Bio-Rad Laboratories B.V, Veenendaal, The Netherlands). The electrophoresis procedure was according to the precast gels manufacturer's instructions. Gels were stained using Coomassie Brilliant Blue (CBB) R-250. Protein concentration was normalized among triplicates 
and samples in a qualitative way by analysing the gel pictures taken with G:BOX Chemi XT4 (Syngene, Cambridge, UK) and using the software GeneSys version 1.5.5.0 (GeneTools version 4.03.01).

\section{In-gel trypsin digestion}

In-gel digestion of proteins and purification of peptides was done following a modified version of a previously described protocol (Rupakula et al., 2013). Disulfide bridges in proteins were reduced by covering the gels with reducing solution $(10 \mathrm{mM}$ dithiothreitol, $\mathrm{pH} 7.6$, in $50 \mathrm{mM} \mathrm{NH}_{4} \mathrm{HCO}_{3}$ ), and the gels were incubated at $60{ }^{\circ} \mathrm{C}$ for $1 \mathrm{~h}$. Alkylation was performed in darkness and shaking (100 rpm) for $1 \mathrm{~h}$ by adding $25 \mathrm{ml}$ of iodoacetamide solution (10 mM iodoacetamide in $100 \mathrm{mM}$ Tris-HCl, pH 8.0). Gels were thoroughly rinsed with demineralized water in between steps. Each gel lane was cut into 3 slices, and the slices were cut into approximately $1 \mathrm{~mm}^{3}$ cubes and transferred to a separate $0.5 \mathrm{ml}$ protein LoBind tube (Eppendorf, Hamburg, Germany). Enzymatic digestion was done with trypsin sequencing grade (Roche, Mannheim, Germany). $100 \mu \mathrm{l}$ of trypsin solution (5 $\mathrm{ng} / \mu \mathrm{l}$ trypsin in $50 \mathrm{mM}$ $\mathrm{NH}_{4} \mathrm{HCO}_{3}$ ) were added to each tube, and incubated 2 hours at $45{ }^{\circ} \mathrm{C}$ with gentle shaking. To stop trypsin digestion, trifluoroacetic acid (10\%) was added to the supernatant to lower the $\mathrm{pH}$ below 5 . The digested protein mixture was purified and concentrated using an in-house made SPE pipette tip (Lu et al., 2011).To recover hydrophobic peptides, $50 \mu \mathrm{l}$ acetonitrile (vol/vol in $0.1 \%$ formic acid) was passed through the column. Finally, the volume was reduced to $20 \mu \mathrm{l}$ using a SpeedVac concentrator and then adjusted to $50 \mu \mathrm{l}$ with $0.1 \%$ formic acid. Samples were analysed using nLC-MS/MS with a Proxeon EASY nLC and a LTQ-Orbitrap XL mass spectrometer as previously described (Lu et al., 2011).

\section{LC-MS data analysis}

The obtained MS/MS spectra were processed with MaxQuant v. 1.5.2.8. Databases with the protein sequences of the organisms involved in the study were downloaded from UniProt (www.uniprot.org). An additional dataset with protein sequences of common contaminants (trypsin, human keratins and bovine serum albumin) was included. False discovery rates (FDR) of less than 1\% were set at peptide and protein levels. Modifications for acetylation (Protein N-term), deamidation (N, Q) and oxidation (M) were allowed to be used for protein identification and quantification. All other quantification settings were kept default. Filtering and further bioinformatics and statistical analysis were performed with Perseus v.1.5.3.0. Proteins included in our analysis contain at least two identified peptides of which at least one is unique and at least one unmodified. Reversed hits and contaminants were filtered out. Protein groups were filtered to require three valid values in at least one experimental group. Label-free quantification (LFQ) intensities (values normalized with respect to the total amount of protein and all its identified peptides) 
were used to analyse the abundance of proteins in the fractions and further statistical comparisons among conditions. LFQ intensities were transformed to logarithmic values base 10. Missing values were imputed with random numbers from a normal distribution, the mean and standard deviation of which were chosen to best simulate low abundance values close to noise level (Width: 0.3 and downshift 1.8 times). A multiple-sample test (ANOVA) with permutation based FDR statistics (250 permutations, $\mathrm{FDR}=0.01$ and $\mathrm{S} 0=1$ ) was applied to filter significant proteins. PCA were performed with default settings and without category enrichment in components. Z-score normalization in which the mean of each row (where each row is a protein in triplicate and in different conditions) is subtracted from each value and the result divided by the standard deviation of the row was applied before clustering. Hierarchical clustering of rows, using Euclidean distances, produced a heat map representation of the clustered data matrix. Row clusters were automatically defined and exported to a new matrix. Imputed values were then replaced back to missing values and previously defined clusters were displayed in a new heat map.

\section{Genome analysis of Methanobacterium formicicum}

Amino acid sequences of protein coding genes for methanogenesis and energy conservation in $M$. hungatei were obtained from the Integrated Microbial Genomes (IMG) system in DOE-Joined Genome Institute (Version 4.560 Mar. 2016). Such sequences were used to retrieve similar functional genes in the genome of $M$. formicicum using the BLAST (Altschul et al., 1990) service of the IMG website. The locus tags assigned to the genes of $M$. formicicum in UniProt are referred to as DSM1535_xxxx, where the $\mathrm{x}$ indicate the gene numbers. For practical purposes in this study we refer to the locus tag of $M$. formicicum as Mfor_xxxx. Amino acid sequences obtained from the best hits were then analysed with InterProScan 5 (version 5RC7, 27th January 2014) to corroborate the presence of key functional domain profiles. TMHMM Server v. 2.0 (Krogh et al., 2001) was used to identify transmembrane helices and the Tat P 1.0 Server of CBS was used to predict twinarginine translocation (Tat) motifs (Bendtsen et al., 2005).

\section{Results and discussion}

\section{Proteomic profiles and most abundant proteins}

The genome of $M$. hungatei predicts 3,239 protein-coding genes (Gunsalus et al., 2016). Our proteome analysis detected 825 proteins, of which 149 proteins are without known function. The core proteome of $M$. hungatei consisted of 625 proteins which were detected in all studied conditions. 186 proteins were detected only in the axenic conditions, namely formate- and hydrogen-grown cells, but not in syntrophically grown cells (Supporting information, Fig. S5.1.A). The number of 
proteins detected in the syntrophic growth condition, only 631, was substantially lower than the amount obtained in the hydrogen and formate conditions with more than 800 proteins each. Considering that the protein extraction was equally successful, and the protein concentration analysed was the same for all studied conditions, this difference in the number of proteins detected was unexpected. This may indicate a more constrained metabolism when the methanogen grows in syntrophy and is limited by the efficiency of the bacterial partner to provide hydrogen, formate and possibly other compounds. The few exclusive proteins for each condition were without predicted function.

The genome of $M$. formicicum has only 2,409 protein-coding genes (Maus et al., 2014). Our study resulted in the detection of 716 proteins of which 117 are with unknown function. The core proteome comprises 574 proteins present in all conditions. 137 proteins were exclusively detected in cells grown with hydrogen or formate, but not in syntrophically grown cells. (Supporting information, Fig. S5.1.B). Similarly, as in $M$. hungatei, the number of proteins obtained from syntrophically grown cells (576) was lower than that obtained from pure cultures. A lower number of proteins detected in syntrophically grown cells indicate, for both methanogens, that during syntrophic growth a restricted set of proteins are produced to perform methanogenesis.

Principal component analysis (PCA) was used to determine the protein abundance variation of the samples according to the different electron donors used for growth: hydrogen, formate or compounds derived from S. fumaroxidans (Figure 5.1). For both methanogens, the first principal component ( $\mathrm{PC} 1 ; \sim 76 \%$ of total variance) clearly separates the proteomic profiles of the syntrophic conditions from those in axenic growth. However, PC1 does not establish a difference between growth on hydrogen or on formate for any of the methanogens. The second principal component in $M$. hungatei ( $\mathrm{PC} 2 ; 7 \%$ of total variance) differentiates the three growth conditions. However, for $M$. formicicum, PC2 does not differentiate between growth with formate and the syntrophically grown cells, with the notable exception of one triplicate of the latter. Nevertheless, PC2 accounts for only $6.3 \%$ of the variability of the data.

\section{Methanogenesis pathway}

All the proteins previously predicted to be involved in the production of methane from hydrogen $+\mathrm{CO}_{2}$ and formate by $M$. hungatei (Gunsalus et al., 2016) were abundant in our study. For $M$. formicicum, we performed our own genomic analysis to manually reconstruct the methanogenic pathway and verified the production of the predicted proteins with the proteomic results. 

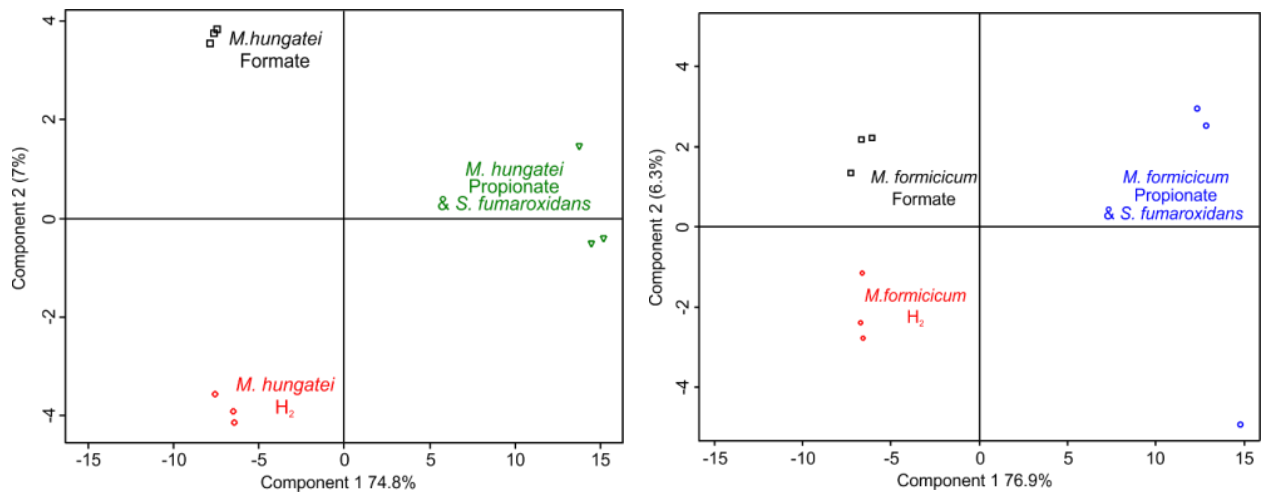

Figure 5.1. Principal Component Analysis performed for Methanospirillum hungatei (left) and Methanobacterium formicicum (right) proteins produced in three growth conditions. Symbols: red diamonds, hydrogenotrophic conditions; black squares, growth with formate; Green triangles and blue circles correspond to the cocultured partnership of $M$. hungatei and $M$. formicicum respectively with Syntrophobacter fumaroxidans.

The first step in methanogenesis from $\mathrm{CO}_{2}$ is catalysed by a formylmethanofuran dehydrogenase. Two isoenzymes have been found in methanogens, a tungstencontaining isoenzyme (Fwd) and a molybdenum-containing isoenzyme (Fmd) (Thauer, 1998). Both isoenzymes present in the thermophilic methanogen Methanothermobacter marburgensis strain Marburg have been purified and studied further (Hochheimer et al., 1995; Hochheimer et al., 1996; Hochheimer et al., 1998). Moreover, genomic and amino acid sequence comparisons have shown that the catalytic subunits of molybdenum isoenzymes $(f m d B)$, such as the one from Methanosarcina barkeri, are more closely related to the molybdenum isoenzymes than to the tungsten isoenzymes from $M$. marburgensis or Methanothermobacter wolfeii (Vorholt et al., 1997). We compared the amino acid sequences of the three catalytic subunits found in M. hungatei (mhun_1983, mhun_1988, mhun_1994) and the one found in $M$. formicicum (Mfor_1495) to all the known $f m d B$ and $f w d B$ sequences used in the analysis of (Vorholt et al., 1997). All the sequences of the catalytic subunits of $M$. hungatei and $M$. formicicum are less than $45 \%$ identical to the molybdenum $f m d B$ from $M$. barkeri, M. marburgensis and M. wolfeii. In contrast, the identities to the tungsten $f w d B$ from $M$. marburgensis, $M$. wolfeii, Methanocaldococcus jannaschii and Methanopyrus kandleri were in all cases above $45 \%$. Although it has been implied that the tungsten isoenzyme $\mathrm{FwdB}$ prevails in thermophilic and hyperthermophilic methanogens (Hochheimer et al., 1998), a tungsten FwdB is presumably present in the mesophilic Methanosarcina acetivorans and M. barkeri (Matschiavelli and Rother, 2015). Therefore, it is possible that $M$. hungatei and M. formicicum contain the tungsten isoforms of formylmethanofuran dehydrogenase. 
Of the three sets of formylmethanofuran dehydrogenases (Fmd/Fwd) in M. hungatei, the enzyme encoded in Mhun_1981-1984 was predominant in all the analysed conditions. The Fmd/Fwd encoded in Mhun_1987-1994 was not detected and only the major subunits of the third Fmd/Fwd (Mhun_2106-2112) were detected in our analysis (Figure 5.2). For $M$. formicicum only one Fmd/Fwd was found encoded in the genome (Mfor_1492-1497) and its proteins were constitutively detected in our analysis. Other minor subunits such as Fmd/Fwd-E, Fmd/Fwd-F and Fmd/Fwd-G were found elsewhere encoded in the genome (Mfor_1518 \& 1521, Mfor_1527 and Mfor_1528 respectively) and abundant in the proteome. Interestingly, subunit Fmd/Fwd-F contains a polyferredoxin that is believed to be the ferredoxin that mediates electron transfer to Fwd/Fmd after bifurcation from the complex Hdr/Mvh (Hochheimer et al., 1995; Costa et al., 2010). Therefore, the production of these kind of proteins might be related to the transfer of electrons in the metabolic processes.

Of the three methenyl-tetrahydromethanopterin (methenyl-H4MPT) cyclohydrolases (Mch) predicted in the genome of M. hungatei, Mch2 (Mhun_0444) was the most abundant and detected in all conditions, while Mch1 (Mhun_0022) was only detected in axenic conditions and Mch3 (Mhun_2384) was not detected at all. In M. formicicum, two formylmethanofuran-H4MPT formyltransferases (Ftr1: Mfor_1101 and Ftr2: Mfor_2022) were detected in all conditions, although Ftr1 was significantly more abundant than Ftr2.

Methylene-H4MPT dehydrogenase (Mtd) and methylene-H4MPT reductase (Mer) were detected in high levels in both methanogens. In $M$. hungatei these enzymes were significantly more abundant in syntrophically grown cells while in $M$. formicicum they were constitutively produced at the studied conditions. Mtd and Mer play an important role in re-oxidation of cofactor $\mathrm{F}_{420}$, and, excluding a couple of histones, were the most abundant proteins together with methyl-coenzyme M reductase (Mcr) in both methanogens.

$M$. formicicum contains two isoenzymes of methyl-CoM reductase: isoenzyme I (McrABG) encoded by the transcription of $M c r A G C D B$ and isoenzyme II (MrtABG) encoded by MrtAGDB (Figure 5.2). Mcr (Mfor_0905-0909) was detected in significantly higher levels in syntrophically grown cells. Mrt (Mfor_1092-1095) on the other hand was not detected in syntrophic conditions, but very high levels were found in axenically grown cells. The transcription of $\mathrm{Mcr}$ and $\mathrm{Mrt}$ is dependent on the growth phase and substrate availability. While $M r t$ is mainly transcribed in the early exponential phase, $\mathrm{Mcr}$ is preferably expressed in the late exponential growth phase (Bonacker et al., 1992; Morgan et al., 1997). We harvested our cells during mid-log phase, so we can only speculate whether the absence of Mrt in syntrophically grown cells with $S$. fumaroxidans corresponds to a slight difference in the time of harvest or to the limited supply of hydrogen or formate during syntrophic conditions. 
In both methanogens subunit McrC of methyl-CoM reductase was not detected, while McrD and MrtD were detected in lower levels than the rest of the methyl-CoM reductase subunits. The function of these subunits is not known, although a role in the activation of the enzyme and in posttranslational modifications, respectively, has been proposed (Prakash et al., 2014; Zheng et al., 2016).

Finally, the membrane-bound H4MPT S-methyltransferase (Mtr) and the soluble heterodisulfide reductase ( $\mathrm{Hdr}$ ) were consistently detected in both methanogens grown at the different conditions. Nevertheless, subunit HdrA (Mhun_1838) in $M$. hungatei was significantly more abundant in syntrophically grown cells. The catalytic site of the heterodisulfide reductase is located in subunit $\mathrm{HdrB}$ (Mhun_1837), but HdrA contains the FAD-binding domain and four [4Fe-4S] clusters that allegedly make this subunit the site where electron bifurcation takes place (Hedderich et al., 1994; Hamann et al., 2007). In the genome of most methanogens, $\mathrm{HdrA}$ is non-adjacent to $\mathrm{HdrBC}$, consistent with the possible use of $\mathrm{HdrA}$ in other complexes besides Hdr (Hedderich et al., 1994; Kaster et al., 2011a). In M. hungatei $\mathrm{HdrA}$ is adjacent to $\mathrm{HdrBC}$, but in $M$. formicicum not. In fact, two genes coding for the HdrA subunit are scattered in the genome in positions Mfor_1232 ( $h d r A 1)$ and Mfor_2055 ( $h d r A 2)$, while $h d r B C$ are in Mfor_0471-72. Although the HdrA1 of $M$. formicicum was equally abundant in all conditions, its levels were significantly higher than the HdrBC complementary subunits. Moreover, HdrA2 was detected only in axenically grown cells. These results in both methanogens suggest that HdrA is used in bifurcating mechanisms of energy conservation in association with other complexes besides HdrBC. Remarkably, in the genome of $M$. hungatei next to $h d r A B C$ the subunit Fwd/Fmd-F is encoded in Mhun_1835 ( $f w d / f m d F)$, which as mentioned before is thought to function as an electron carrier between Hdr and Fwd/Fmd. FwdF was abundant in all growing conditions of M. hungatei.

Figure 5.2. Protein expression heat map of the of the proteins used in methanogenic pathways of Methanospirillum hungatei JF1 (left) and Methanobacterium MFOR (right). The rows in the heat map show proteins levels after $\mathrm{Z}$-score standardization in three different growth conditions. The columns show from left to right, in triplicates, the electron donor used by the microorganisms to produce methane: formate, hydrogen and interspecies compounds transferred from Syntrophobacter fumaroxidans. The colour scale indicates the degree of protein down- or up regulation ranging from blue (-2.5 log ratio), to yellow (2.5 log ratio). The colour intensities indicate lower and higher levels compared to the average level 0 value (in black); the grey colour represents not detected. Fwd/Fmd: formylmethanofuran dehydrogenase; Ftr: formylmethanofuran-H4MPT formyltransferase; Mch: methenyl-H4MPT cyclohydrolase; Mtd: methylene-H4MPT dehydrogenase; Mer: methylene-H4MPT reductase; Mtr: membrane bound H4MPT S-methyltransferase; Mcr \& Mrt: methyl-CoM reductase isoenzyme I \& II respectively; Hdr: heterodisulfide reductase; and Mvh: F 420 -non-reducing hydrogenase. $\left(^{*}\right)$ indicates a statistically significant difference in at least one condition. Subunits are indicated after the locus tag. 


\begin{tabular}{|c|c|c|c|c|c|c|c|}
\hline & & Methan & ospirillum hungatei & & Methanol & bacterium & ormicicum \\
\hline & & -2.5 & 2.5 & & -2.5 & 0 & 2.5 \\
\hline Protein & $\begin{array}{c}\text { Genome } \\
\text { Reference }\end{array}$ & \begin{tabular}{|c|} 
Formate \\
R1 R2 R3
\end{tabular} & $\begin{array}{ll}\text { Hydrogen } & \text { Syntrophy } \\
\text { R1 R2 R3 R1 R2 R3 }\end{array}$ & $\begin{array}{c}\text { Genome } \\
\text { Reference }\end{array}$ & \begin{tabular}{|l} 
Formate \\
R1 R2 R3
\end{tabular} & $\begin{array}{l}\text { Hydroger } \\
\text { R1 R2 R }\end{array}$ & $\begin{array}{l}\text { Syntrophy } \\
\text { R1 R2 R3 }\end{array}$ \\
\hline & Mhun_1981 C & & & Mfor_1492 F & & & \\
\hline & Mhun_1982 A & & & Mfor_1493 G & & & \\
\hline & Mhun_1983 B & & & Mfor_1494 D & & & \\
\hline & Mhun_1984 D & & & Mfor_1495 B & & & \\
\hline & Mhun_1987 & & & Mfor_1496 A & & & \\
\hline Fwd & to & & DT DETECTED & Mfor_1497 $C^{\star}$ & & & \\
\hline 1 & Mhun_1994 & & & & & & \\
\hline$I$ & Mhun_2106 G & & & & & & \\
\hline Fmd & Mhun_2107 D & & & Mfor $1518 E^{*}$ & & & \\
\hline & Mhun_2108 B & & & Mfor_1521E & & & \\
\hline & Mhun_2109 A & & & Mfor_1527 F & & & \\
\hline & Mhun_2110 & & & Mfor_1528 $G^{*}$ & & & \\
\hline & Mhun_2112 $C^{*}$ & & & & & & \\
\hline & & & & Mfor_1101 & & & \\
\hline Ftr & |Mnun_T808" & & & Mfor 2022 & & & \\
\hline & Mhun_0022* & & & & & & \\
\hline Mch & Mhun_0444 & & & Mfor_2390* & & & \\
\hline & Mhun_2384 & & गT DETECTED & & & & \\
\hline Mtd & Mhun_2255* & & & Mfor_1363 & & & \\
\hline Mer & Mhun_2257* & & & Mfor_0704 & & & \\
\hline & Mhun_2168E & & & Mfor_0897 H & & & \\
\hline & Mhun_2169 $D^{*}$ & & & Mfor_0898 G & & & \\
\hline & Mhun_2170 C & & & Mfor_0899 F & & & \\
\hline Mtr & Mhun_2171 B & & & Mfor_0900 A & & & \\
\hline . & Mhun_2172 A & & & Mfor_0901 $B^{\star}$ & & & \\
\hline & Mhun_2173 F & & & Mfor_0902 C & & & \\
\hline & Mhun_2174 G/A & & & Mfor_0903 D & & & \\
\hline & Mhun $2175 \mathrm{H}$ & & & Mfor_0904E & & & \\
\hline & Mhun_2144 $B^{\star}$ & & & Mfor_0905 $A^{*}$ & & & \\
\hline & Mhun_2145 $D^{*}$ & & & Mfor_0906 $G^{*}$ & & & \\
\hline Mcr & Mhun_2146 C & & & Mfor_0907 C & & & \\
\hline & Mhun_2147 $G^{*}$ & & & Mfor_ $0908 D^{*}$ & & & \\
\hline & Mhun_2148 $A^{*}$ & & & Mfor_0909 $B^{*}$ & & & \\
\hline & & & & Mfor_1092 $B^{*}$ & & & \\
\hline Mrt & & & & Mfor_1093 $D^{\star}$ & & & \\
\hline & & & & Mfor_1094 $G^{\star}$ & & & \\
\hline & & & & Mfor_1095 $A^{*}$ & & & \\
\hline & Mhun_1835 fwdF & & & Mfor_2055 $\boldsymbol{A}^{*}$ & & & \\
\hline He & Mhun_1836 C & & & Mfor_0471 B & & & \\
\hline Har & Mhun_1837 B & & & Mfor_0472 C & & & \\
\hline & Mhun_1838 $\boldsymbol{A}^{*}$ & & & Mfor_1232 A & & & \\
\hline & Mhun_1839 D & & & Mfor_0880 B & & & \\
\hline Mvh & & & & Mfor_0881 A & & & \\
\hline & & & & Mfor_0882 G & & & \\
\hline & & & & Mfor_0883 D & & & \\
\hline
\end{tabular}




\section{Role of formate and hydrogen in methanogenesis}

Insight into the mechanism by which methanogens oxidize hydrogen or formate is important from the perspective of the use of these compounds as electron carriers in the metabolic processes and for energy conservation. In hydrogenotrophic methanogens like $M$. hungatei and $M$. formicicum formate and hydrogen are the electron donors that generate reduced ferredoxin and cofactor $\mathrm{F}_{420}$ that are used in diverse steps of the methanogenic pathway (Supporting information, Fig. S5.3 \& S5.4). There are indications that hydrogen is formed when methanogens grow on $\mathrm{CO}$ and formate (Costa et al., 2013a; Diender et al., 2016). The need of hydrogen to start methanogenesis from $\mathrm{CO}_{2}$ can be explained by its role in the so called Wolfe cycle (Thauer, 2012), a flavin-based electron bifurcation mechanism that links the initial reduction of $\mathrm{CO}_{2}$ by $\mathrm{Fwd} / \mathrm{Fmd}$ with a multi-complex formed by $\mathrm{HdrABC}$ and an $\mathrm{F}_{420}$ non-reducing hydrogenase (MvhADG) (Setzke et al., 1994; Stojanowic et al., 2003; Hedderich et al., 2005). However, many members of the Methanomicrobiales lack the genes coding for MvhA and MvhG (Thauer et al., 2010). That is the case for $M$. hungatei, where only muhD (Mhun_1839) is found in the genome adjacent to hdrABC (Figure 5.2). MvhD was abundant in all growing conditions of M. hungatei.

It has been suggested that the subunits FrhAG of a coenzyme $\mathrm{F}_{420}$-dependent [NiFe]hydrogenase (FrhADGB) are used instead of MvhAG to form a functional complex with the MvhD subunit and HdrABC (Anderson et al., 2009; Kaster et al., 2011a). Indeed, the FrhADGB of $M$. hungatei (Mhun_2329-2332) was abundant in all conditions and the most abundant hydrogenase in this methanogen (Figure 5.3). $M$. formicicum on the other hand, is a member of Methanobacteriales and contains a complete MvhADGB (Mfor_0880-0883) which was also abundant in all conditions. Therefore, it is possible that MvhADGB in $M$. formicicum and FrhADGB in $M$. hungatei are the enzymes involved in the multi-subunit bifurcating complex that with $\mathrm{HdrABC}$ couples the exergonic reduction of CoM-S-S-CoB to the unfavourable reduction of ferredoxin with $\mathrm{H}_{2}$ (Kaster et al., 2011b).

Figure 5.3. Normalized expression matrix of hydrogenases and formate dehydrogenases of Methanospirillum hungatei JF1 (left) and Methanobacterium formicicum MFOR (right). Protein abundance levels are shown after Z-score normalization. The detected proteins are shown for three different growth conditions. The columns show from left to right, in triplicates, the electron donor used by the microorganisms: formate, hydrogen and interspecies compounds from Syntrophobacter fumaroxidans. $(*)$ marks a statistically significant difference in at least one condition. The colour intensity indicates the degree of protein up- or down regulation compared to the average level value in black; the grey colour is used for not detected proteins. 


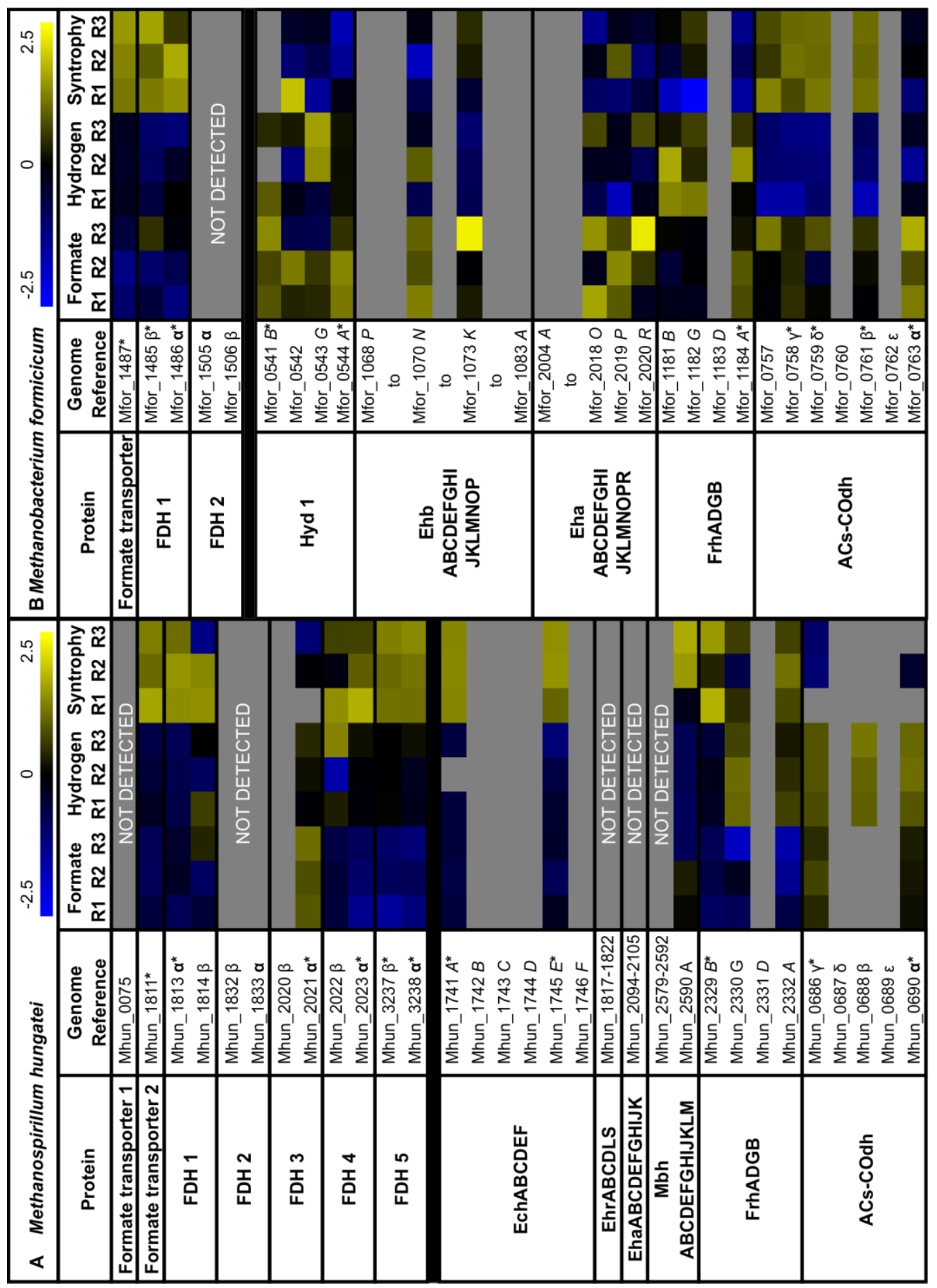


During growth on formate electrons may flow from formate to $\mathrm{Hdr}$ using an $\mathrm{F}_{420^{-}}$ reducing formate dehydrogenase instead of the $\mathrm{F}_{420}$-reducing hydrogenase (Costa et al., 2010). For this reason, $\mathrm{H}_{2}$ is not required as intermediate in methanogenesis from formate (Lupa et al., 2008).

In $M$. formicicum only $f d h 1$ (Mfor_1485-1486) codes for cofactor $\mathrm{F}_{420}$ binding domains. Fdh1 was detected in all conditions in $M$. formicicum, and significantly more abundant in syntrophically grown cells. Fdh2 was not detected in any growth conditions in our study. The amino acid sequence of this enzyme, fdh2 (Mfor_15051506), does not predict cofactor $\mathrm{F}_{420}$ binding domains. In the case of $M$. hungatei, the five formate dehydrogenases present in its genome are nearly identical (Gunsalus et al., 2016), and all of them contain cofactor $\mathrm{F}_{420}$ binding motives in their amino acid sequences. Therefore, all of them could in theory be used in the Wolfe cycle, although Fdh1 (Mhun_1813-1814) was the most abundant formate dehydrogenase in all conditions and significantly more abundant in syntrophically grown cells.

When methanogens use formate as substrate, it is first imported inside the cell by the formate transporter, then it is oxidized by a formate dehydrogenase to generate reduced cofactor $\mathrm{F}_{420}\left(\mathrm{~F}_{420} \mathrm{H}_{2}\right)$ which is required in several steps of methanogenesis. In the genome of both methanogens genes coding for Fdh1 (Mhun_1813-1814 \& Mfor_1485-1486) are adjacent to their formate transporter coding gene (Mhun_1811 \& Mfor_1487). These formate transporters and formate dehydrogenases were among the most abundant proteins detected in both methanogens. Therefore, the Fdh1 in each methanogen, is most probably the main formate dehydrogenase used to generate $\mathrm{F}_{420} \mathrm{H}_{2}$ necessary for the intermediate reduction steps in methanogenesis performed by Mtd and Mer. Moreover, significantly higher levels of the formate transporter and the associated Fdh1 were detected in syntrophically grown cells of both methanogens in comparison with axenic conditions. This indicates that formate was an important compound provided from Syntrophobacter fumaroxidans.

In M. hungatei, Fdh2 (Mhun_1832-1833) was not detected. Interestingly, Fdh3 (Mhun_2020-2021) was the only formate dehydrogenase that was significantly more abundant in formate-grown cells. Thus, it might be that it has a similar function as Fdh1. Fdh4 (Mhun_2022-2023) and Fdh5 (Mhun_3237-3238) were more abundant in syntrophically grown cells. Fdh5 was the second most abundant formate dehydrogenase after Fdh1 and followed by Fdh4. We suggest that Fdh4 and Fdh5 associate with HdrA, which is also more abundant in syntrophic cultures, to form bifurcating protein complexes for energy conservation at energy-limited conditions such as during syntrophic growth.

Of the five hydrogenases predicted from the genome of $M$. hungatei, only two were detected in our proteome study, Frh and Ech. Although not all the subunits of the energy-conserving hydrogenase (Ech) were detected, the most important parts 
corresponding to the catalytic subunit (EchE: Mhun_1745) and the major membrane integrated subunit (EchA: Mhun_1741) were detected. Consequently, the complex was considered functional. This was not the case for the membrane-bound hydrogenase, where only the alpha-subunit of the complex (MbhA: Mhun_2590) was detected in low levels. However, in previous transcriptomic studies the transcription levels of this complex were higher than those of Ech in all tested conditions (Worm et al., 2011b). Furthermore, our results contrary to the transcriptomics study show a significant higher abundance of Ech in syntrophic conditions. Although an important anaplerotic role of energy-converting hydrogenase Eha in hydrogenotrophic methanogens was proposed (Lie et al., 2012), this membranebound complex was not detected in the present study and it was not considered in the previous transcriptomic study.

In contrast to $M$. hungatei, all the hydrogenases found in the genome of $M$. formicicum were detected in our analysis. Although only few subunits of the multimeric energy-converting hydrogenases Eha and Ehb were detected, these corresponded to the active sites of the complexes. Therefore, we categorized the enzymes as being produced. In $M$. formicicum, also Frh was the most abundant hydrogenase in all studied conditions, excluding Mvh which has been discussed above. The relative abundance of all the hydrogenases remains constant among the studied conditions, and only the alpha-subunits of the Hyd1 and Frh showed a significant increase in cells that were grown axenically. Also, in both axenic conditions MvhADGB is the most abundant energy conservation protein, and only in syntrophically grown cells the Fdh1 and the formate transporter were more abundant than MvhADGB.

In a previous transcriptomic analysis, no main differences in the transcriptional levels of hydrogenases or formate dehydrogenases of $M$. hungatei were observed between cells grown syntrophically or grown with hydrogen or formate (Worm et al., $2011 b)$. It has been documented that the propionate degradation rate of $S$. fumaroxidans in coculture with $M$. formicicum was lower than that in the coculture with $M$. hungatei, and that this might be related to the $\mathrm{Km}$ values of the formate dehydrogenases of the methanogenic partners as well as their formate threshold values (Dong et al., 1994).

A proteomic study of propionate degradation by $S$. fumaroxidans in axenic conditions and in syntrophy with $M$. hungatei and $M$. formicicum (Sedano-Nunez et al., unpublished), revealed that formate is the preferred carrier by the bacterium in the interspecies electron transfer to the syntrophic partner. The present study indicates that $M$. hungatei mainly uses formate dehydrogenases in its methanogenic metabolism, while most of its multiple hydrogenases seemingly are not used, or at least were not detected in our study. In contrast, $M$. formicicum relies on its 
hydrogenases regardless the electron donor available, which in the syntrophic association with $S$. fumaroxidans it is likely formate.

Carbon assimilation, autotrophy in M. formicicum vs acetate dependence in M. hungatei

Hydrogenotrophic methanogens can assimilate carbon via acetyl-CoA generated from methyl-H4MPT. The reductive acetyl-CoA pathway includes a reduction of $\mathrm{CO}_{2}$ to $\mathrm{CO}$, which is subsequently combined with methyl-H4MPT and CoA-SH to form acetyl-CoA. The key enzyme that performs these reactions is therefore referred to as CO dehydrogenase-acetyl-CoA synthase (ACs-COdh) (Berg et al., 2010). Both $M$. hungatei and $M$. formicicum encode in their genome ACs-COdh (Mhun_0686-0690 and Mfor_0757-0763, respectively). The role of ACs-COdh in M. hungatei is unknown since the archaeon, despite having the necessary genes to fix carbon, needs to acquire acetate supplied in the medium as the major supply for cell carbon (Ferry and Wolfe, 1977). As can be seen in Figure 5.3 the ACs-COdh of M. hungatei was scarcely detected. It might be argued that ACs-COdh was not produced by $M$. hungatei since acetate was supplemented in the medium. However, the same medium supplied with acetate was used to grow $M$. formicicum, in which high levels of detection of the ACsCOdh complex in all conditions indicates a role in assimilatory metabolism. The epsilon subunit of the complex and the COdh maturation protein (Mfor_0760) were not detected in any condition. Interestingly these subunits have been found in higher abundance in $M$. marburgensis when performing CO-oxidation (Diender et al., 2016). Since in our study CO oxidation does not take place, these subunits were absent in the proteome.

\section{Other abundant proteins in all growth conditions}

The total intensity-based absolute quantification (iBAQ) was used to rank the most abundant proteins produced in all growth conditions. Proteins associated with protection, transport and stabilization of other proteins and macromolecules are commonly abundant in several microbial proteomic studies (Ishihama et al., 2008; Moriya, 2015). This is also the case for our proteomic analysis of M. hungatei and $M$. formicicum.

In $M$. hungatei, among the most abundant proteins was an uncharacterized protein (Mhun_2513) containing a domain of unknown function DUF3821 (IPR024277). This domain is largely confined to sequences from Methanomicrobiales and found in putative lipases, but the function is still unknown (InterPro, 5RC7, 27 th January 2014). Two other proteins (Mhun_1218 \& Mhun_3140) that are related to the formation of archaeal pili and archaeal flagella were also among the most abundant proteins in all the studied conditions of $M$. hungatei. Previous genomic analysis predicted the presence of a basal body structure in the flhGFHIJ (Mhun_0101-0105) 
gene cluster in M. hungatei (Gunsalus et al., 2016). However, only one subunit of the flhGFHIJ complex was detected at low levels in our study (Figure 5.4).

The extracellular filament structure formerly called archaeal flagellum is now referred to as archaellum (Jarrell and Albers, 2012). In addition to motility, the archaella are involved in cellular adhesion, biofilm formation and symbiotic interactions such as cell-cell contact (Bellack et al., 2011; Jarrell et al., 2011). Of the three genes coding for the archaella filaments in M. hungatei, only the product of Mhun_3140 was detected. This protein was among the most abundant in all conditions, and not only in syntrophic grown cells, therefore a role of cell-cell interaction with the syntrophic partner is not likely, although motility or other type of adhesion are still feasible traits. The atomic model of this specific archaellum protein indicates that archaella exhibit similarities to both bacterial flagella and bacterial type IV pili (Poweleit et al., 2016).

Besides the archaella structures, the genome of $M$. hungatei reveals the presence of 12 genes coding for Archaeal type IV pili. Archaeal pili also play important roles in surface adhesion and they could also play a role to establish cell to cell interactions with the syntrophic partner (Esquivel et al., 2013). Although these appendages have never been observed by electron microscopy, the product of one of these paralogs (Mhun_1218) was the fourth most abundant protein in M. hungatei, and it was consistently present in the three studied conditions. Interestingly, the other four paralogs (Mhun_0296-0299) were detected at high levels in cells grown axenically but were not detected in syntrophically grown cells. Lastly two more paralogs (Mhun_0310-0311) were retrieved in all conditions, but these were significantly more abundant in axenic conditions (Figure 5.4). These results indicate that the function of these archaeal pili, as in the case of the archaella, is not dependent on syntrophic associations, but they might still be linked to cell-cell interactions among the methanogens themselves.

Other proteins that could be involved in regulating cell adhesion are surface layer proteins (SLP or S-layer proteins). In Methanosarcina mazei and M. acetivorans SLP's are thought to regulate cell adhesion and it was found that beta-propeller, PKD, and beta-helix domains account for the complete architecture of numerous SLPs in those methanogens (Jing et al., 2002). Many hypothetical proteins containing these domains were found in the genome of M. hungatei (Mhun_2440-42; Mhun_0417-0426). Moreover, several of those proteins ranked high in the total iBAQ values.

In contrast, in the genome of $M$. formicicum there are no genes coding for proteins containing PKD, beta-helix or beta-propeller domains. This seems reasonable since, with the exception of Methanothermus fervidus, there are no reports of the presence of an S-layer in the other known members of the Methanobacteriales (Albers and 
Meyer, 2011). In this order a polymer similar to bacterial peptidoglycan, known as pseudomurein, is the predominant compound in the cell wall (Steenbakkers et al., 2006; Visweswaran et al., 2011).

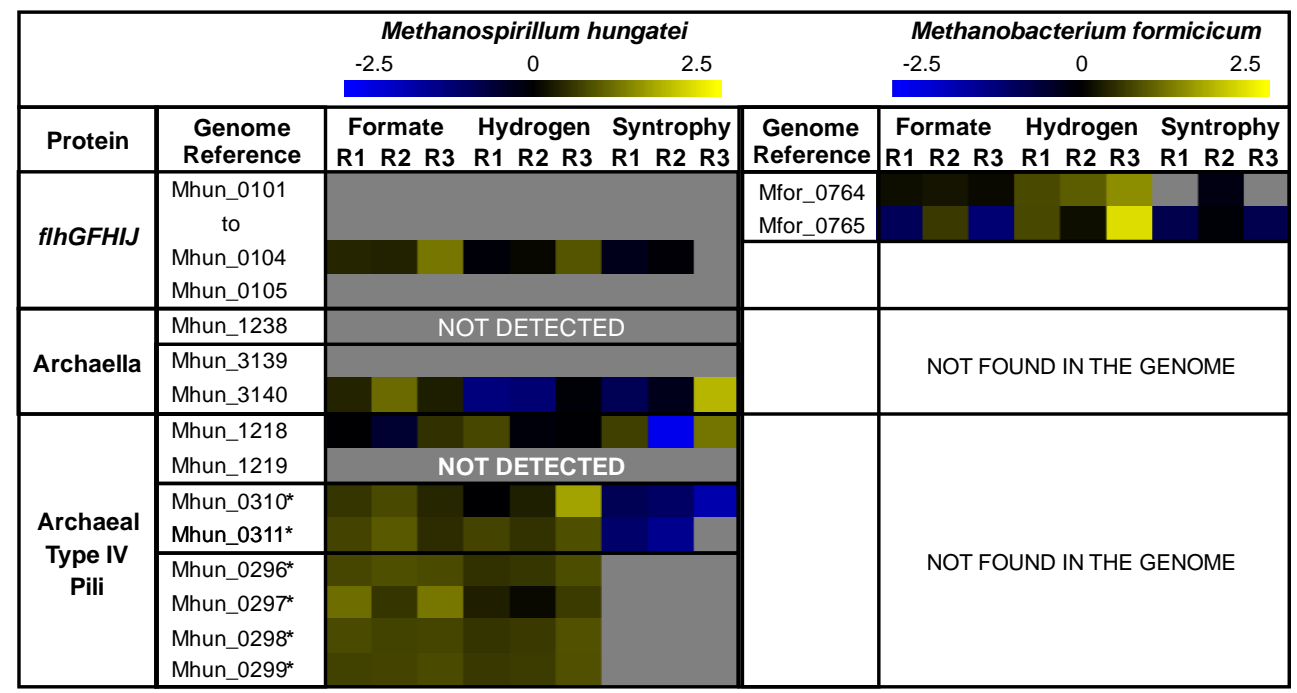

Figure 5.4. Normalized expression matrix of proteins involved in cell-surface structures of Methanospirillum hungatei and Methanospirillum formicicum. The rows in the heat map show the detected proteins after Z-score standardization in three different growth conditions. The columns show from left to right, in triplicates, the electron donor used by the microorganisms: formate, hydrogen and interspecies compounds from Syntrophobacter fumaroxidans. (*) marks a statistically significant difference in at least one condition. The colour intensity indicates the degree of protein up- or down regulation compared to the average level value in black; the grey colour is used for not detected proteins

\section{Conclusions}

The higher abundance of the formate transporter and Fdh1 in syntrophically grown cells of $M$. hungatei and $M$. formicicum strongly indicates that interspecies electron transfer via formate occurred during syntrophic growth with Syntrophobacter fumaroxidans and that this bacterium mainly produces formate in the conversion of propionate.

M. formicicum uses a $\mathrm{F}_{420}$-non-reducing hydrogenase (MvhADG) for bifurcation in the Wolfe cycle while $M$. hungatei employs a $\mathrm{F}_{420}$-reducing hydrogenase (FrhADGB). In syntrophic growth, a $\mathrm{F}_{420}$-dependent formate dehydrogenase is used in both methanogens for electron bifurcation.

We also propose that in M. hungatei, Fdh4 and/or Fdh5 can form a bifurcating complex with HdrA independent of the Wolfe cycle and that these complexes are necessary at energy-limited growth conditions, such as during syntrophic growth. 
Moreover, we speculate that $M$. hungatei uses formate in its metabolic processes regardless whether hydrogen or formate is the substrate. This consideration is based on the number of formate dehydrogenase genes present in its genome and the abundance of formate dehydrogenases even when hydrogen is the substrate, whereas many available hydrogenases were not abundant in our studied conditions. The higher abundance of Mtd and Mer in syntrophically grown cells in M. hungatei, fits with the abundance of cofactor $\mathrm{F}_{420}$ depending formate dehydrogenases Fdh1, Fdh4 and Fdh5 also more abundant in M. hungatei cells grown with S. fumaroxidans.

In $M$. formicicum on the other hand, for reduction of $\mathrm{CO}_{2}$ to methane seems to preferentially use hydrogen as electron carrier since all the hydrogenases in catabolic (Mvh, Frh and Hyd1) and anabolic reactions (EhA and EhB) were equally abundant in cells grown with hydrogen, formate or in syntrophy with S. fumaroxidans. Still the use of formate remains important in some conditions such as syntrophic growth with S. fumaroxidans. 
Supporting information

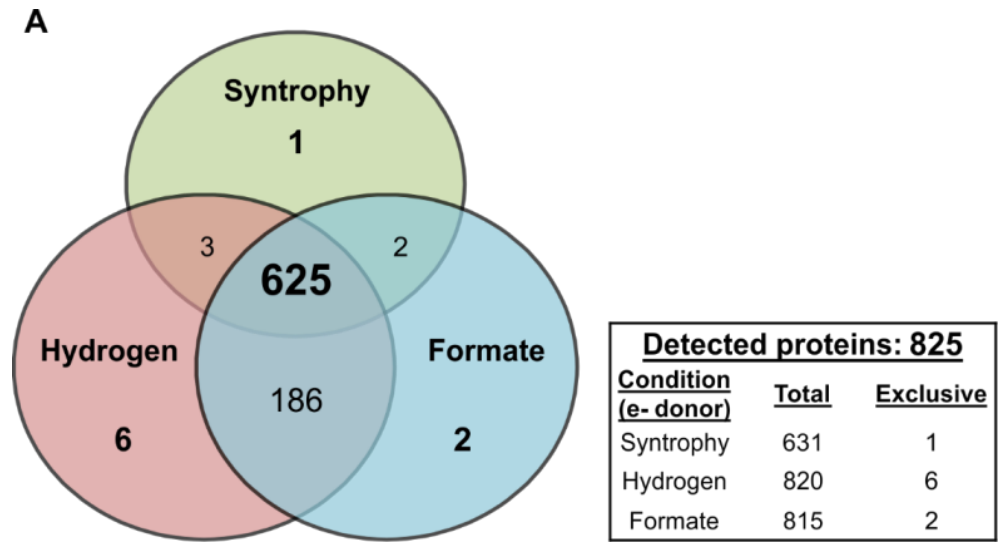

B

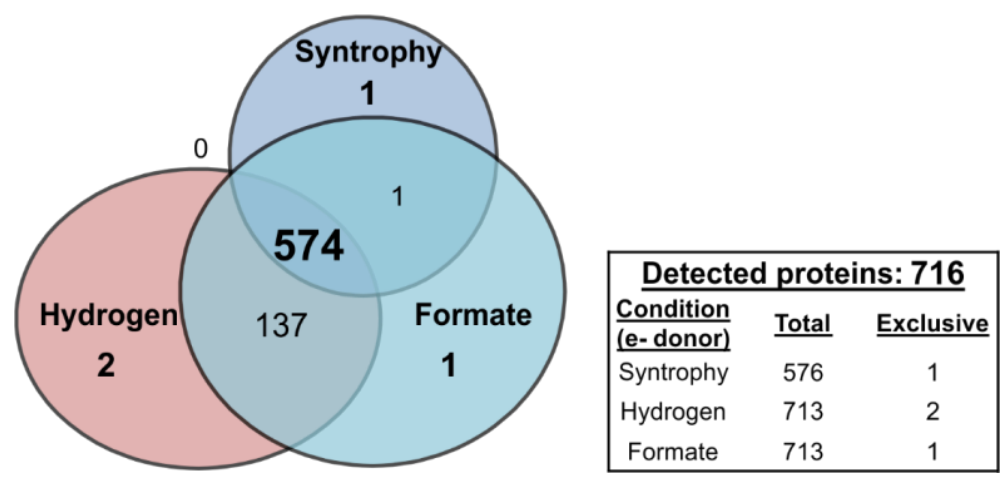

Fig. S5.1. Venn diagrams of the proteomic profiles of Methanospirillum hungatei (A) and Methanobacterium formicicum (B) growing syntrophically with Syntrophobacter fumaroxidans and axenically on $\mathrm{H}_{2} / \mathrm{CO}_{2}$ or formate. 

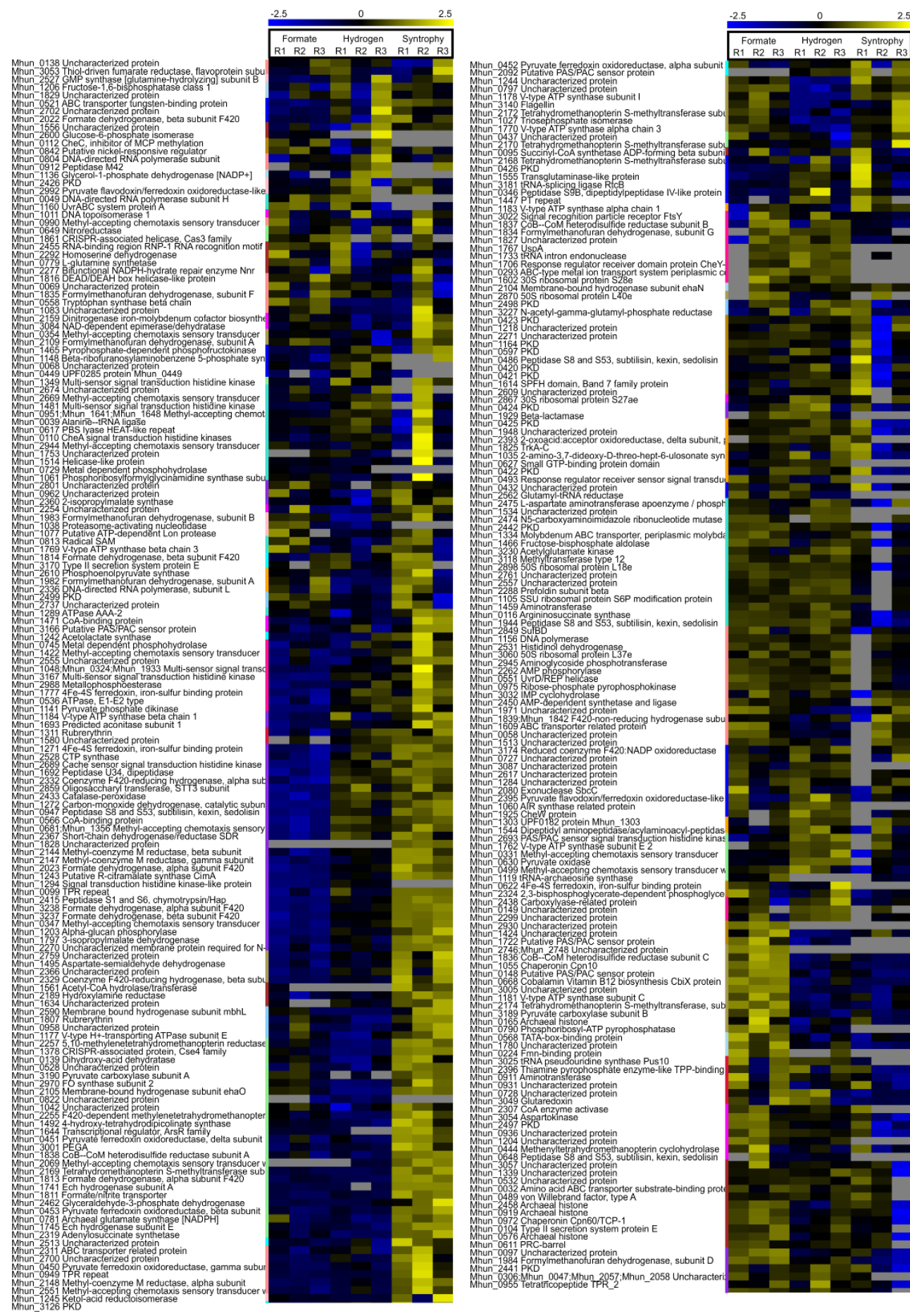


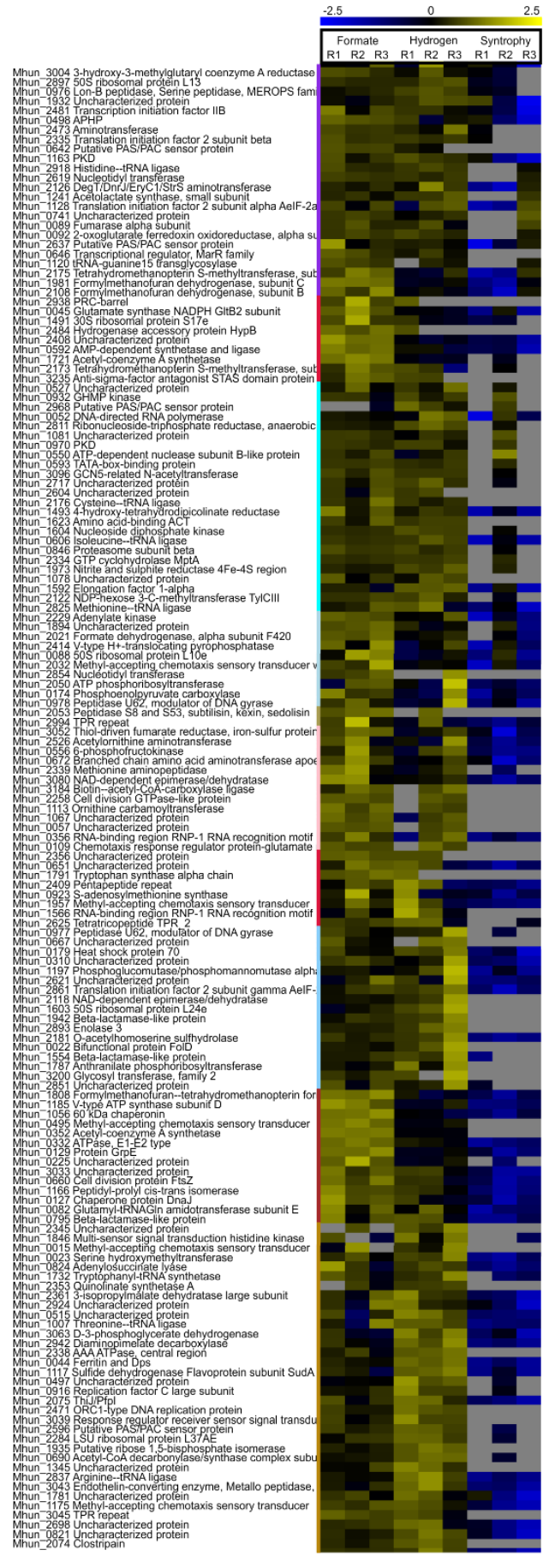

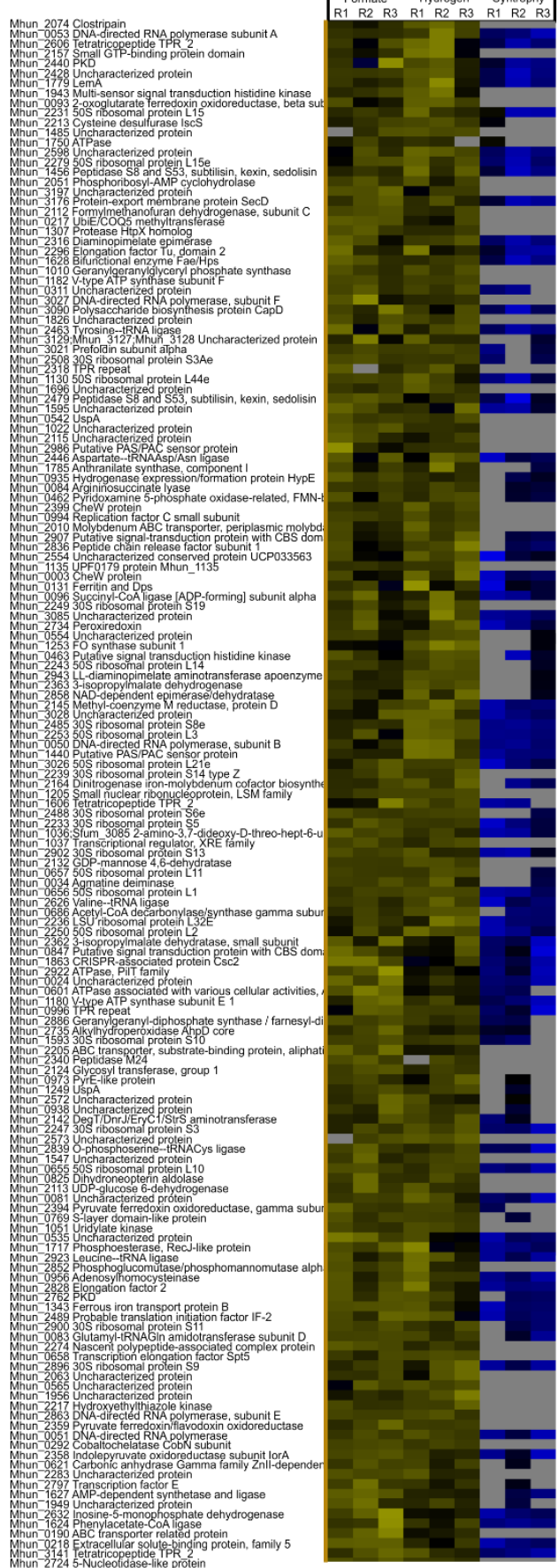




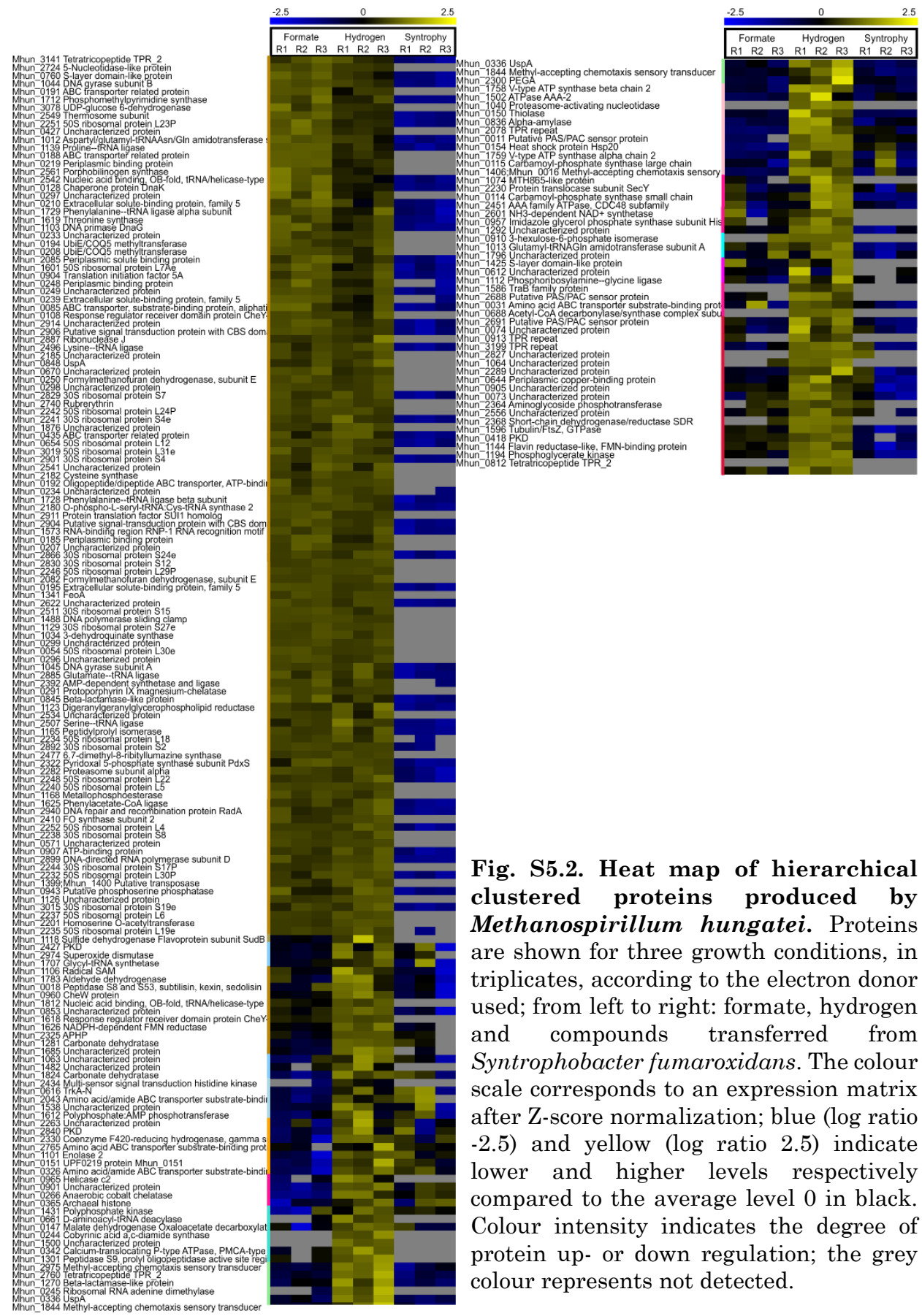



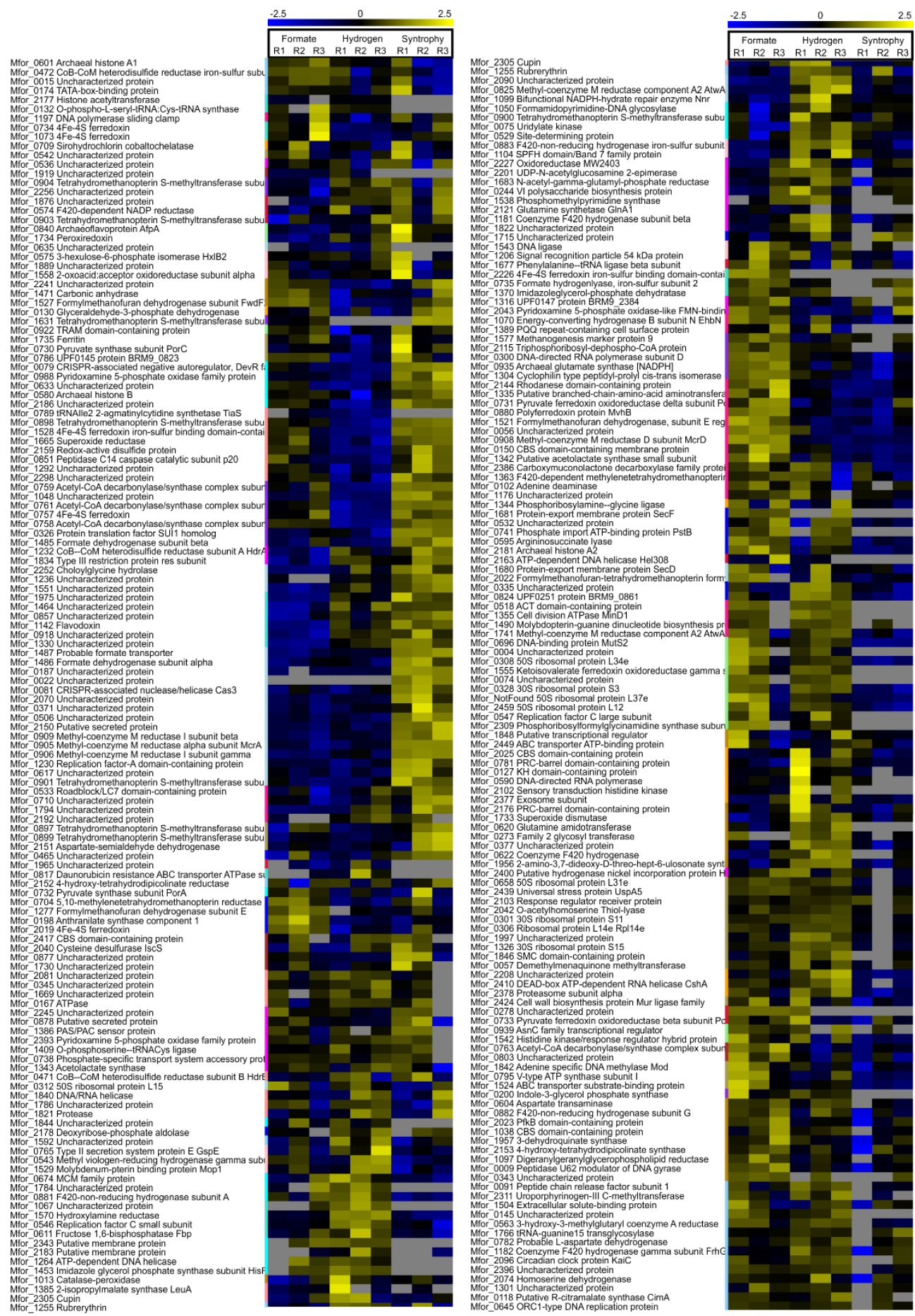


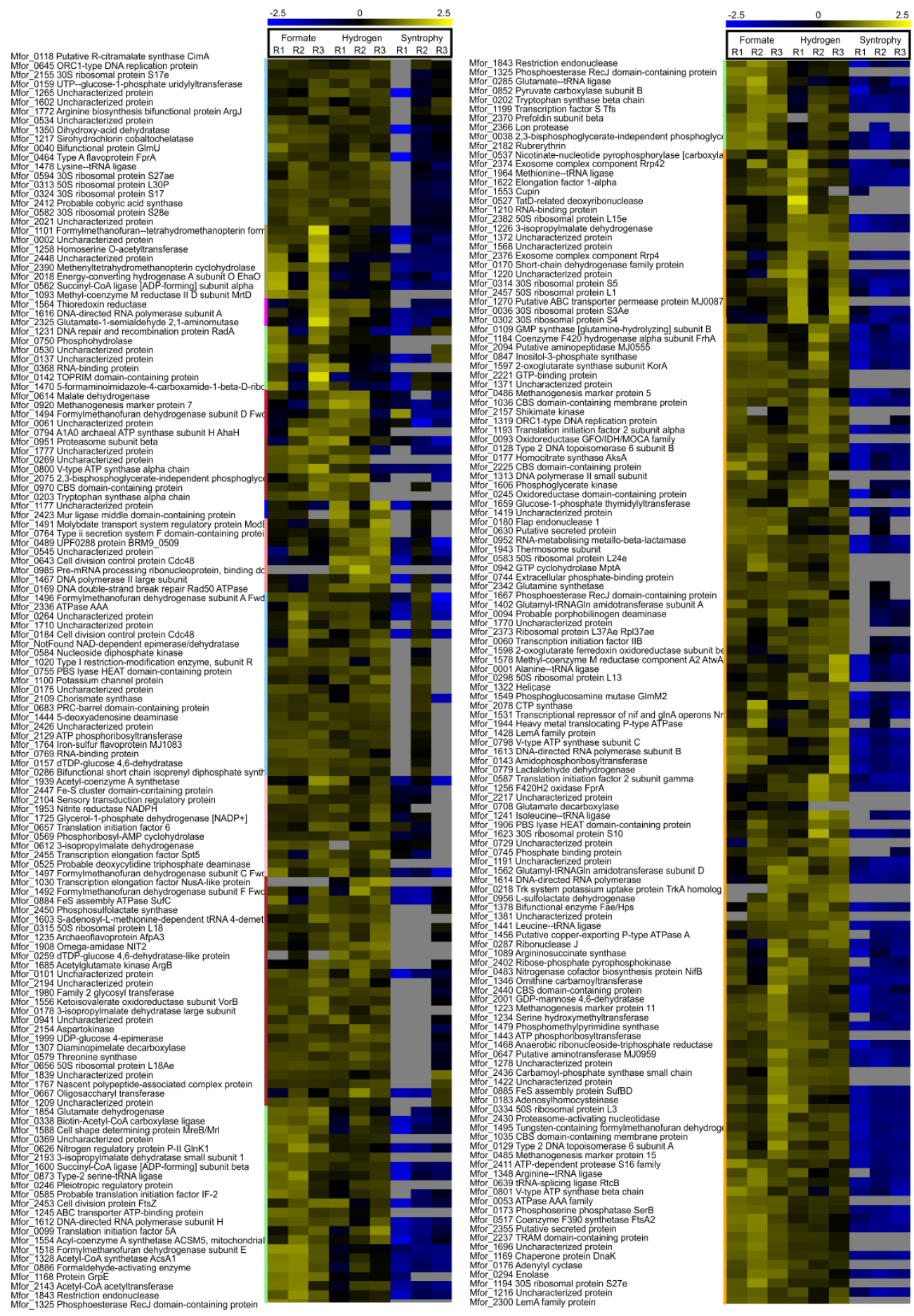




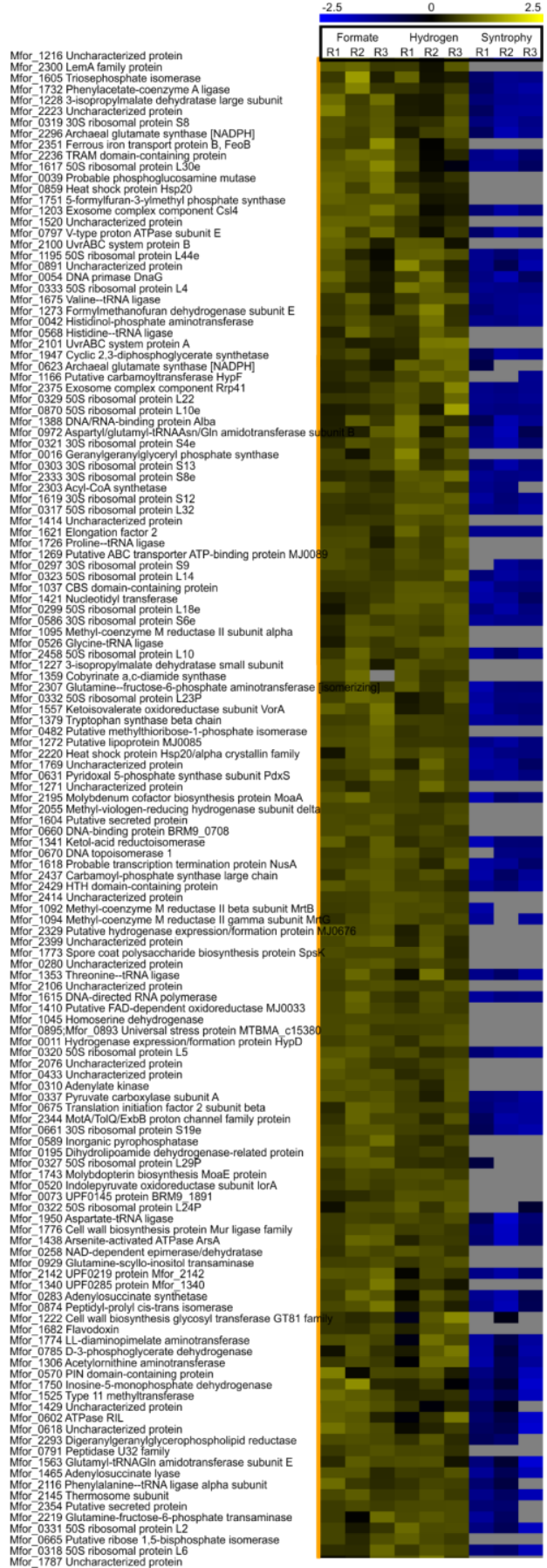

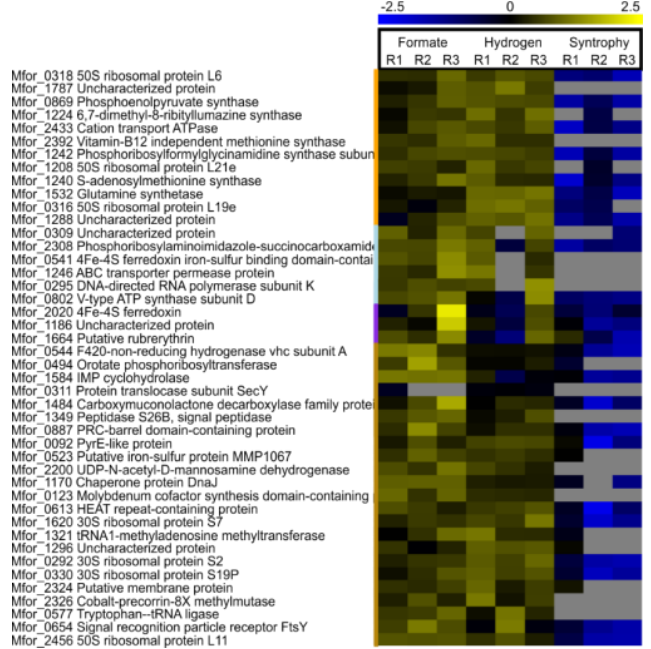

Fig. S5.3. Heat map of hierarchical clustered proteins produced by Methanobacterium formicicum. Proteins are shown for three growth conditions, in triplicates, according to the electron donor used; from left to right: formate, hydrogen and compounds transferred from Syntrophobacter fumaroxidans. The colour scale corresponds to an expression matrix after Z-score normalization; blue (log ratio -2.5) and yellow (log ratio 2.5) indicate lower and higher levels respectively compared to the average level 0 in black. Colour intensity indicates the degree of protein up- or down regulation; the grey colour represents not detected.

Fig. S5.5. Overview of the methanogenic pathways in Methanobacterium formicicum and in Methanospirillum hungatei. 


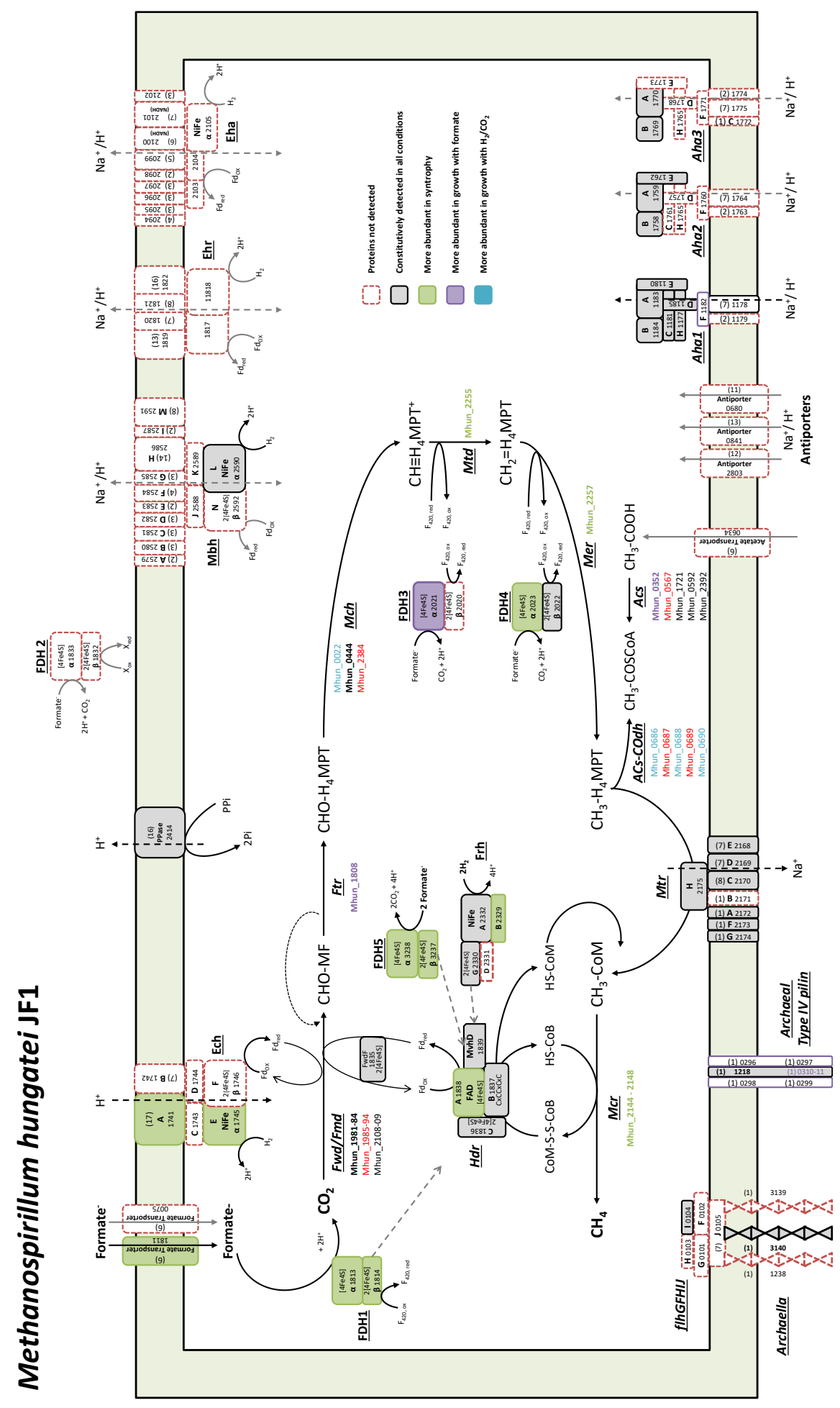




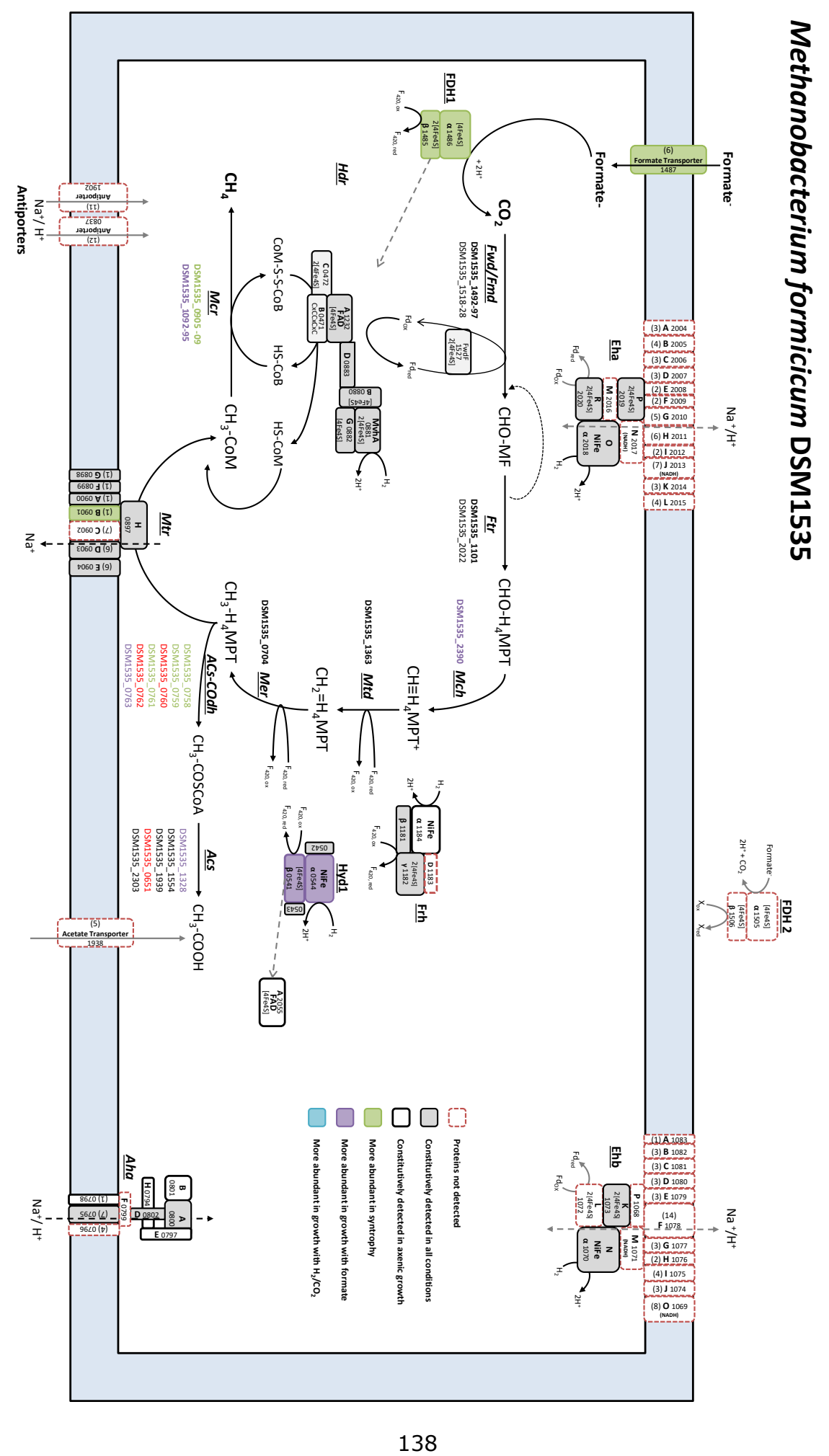


"Genome sequencing has changed taxonomy" Richard Dawkins 

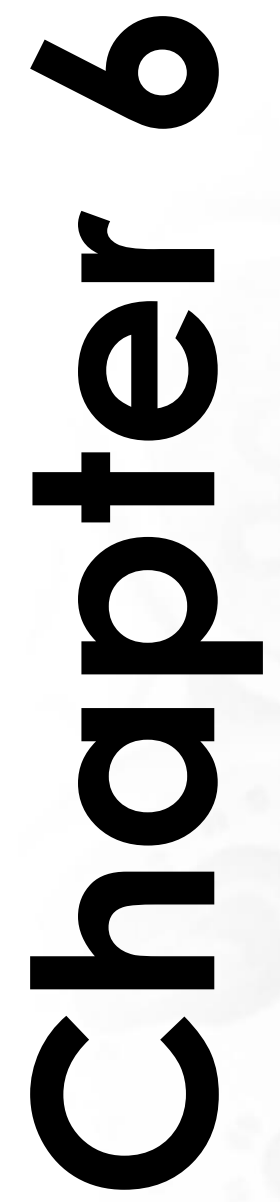


\title{
CHAPTER 6
}

\section{Microbial community analysis and performance of a mesophilic anaerobic membrane bioreactor treating pot ale}

\begin{abstract}
Sedano-Núñez V.T. ${ }^{1}$, Van der Zee F.P. ${ }^{2}$, Ollier J. ${ }^{3}$, Stams A.J.M. ${ }^{1}$ and Plugge C.M. ${ }^{1}$
${ }^{1}$ Laboratory of Microbiology, Wageningen University and Research, The Netherlands ${ }^{2}$ Biothane Systems Intl/Veolia Water Solutions \& Technologies, Delft, The Netherlands ${ }^{3}$ Air Liquide, Grenoble, France.
\end{abstract}

In preparation for publication 


\begin{abstract}
In this study we evaluated start-up, performance and robustness to high loading tests of an anaerobic membrane bioreactor (AnMBR). A 10-liter, completely stirred anaerobic bioreactor in combination with an ultrafiltration cross flow module was operated at $37^{\circ} \mathrm{C}$ for 242 days. The reactor was fed with pot ale from a whiskey distillery in Scotland. High loading tests (HLT) via short time increases of volumetric loading rate (VLR) were applied to assess the robustness of the biomass and monitor biological and biochemical responses to such disturbances. The reactor was rather robust in performance and recovered from 2 and 3 -fold increases in VLR for up to 6 hours. Hydrogen concentrations were constantly measured and showed an increase in response to the HLT in parallel to the increase of total-VFA in the permeate. Population dynamics in the reactor was monitored by Illumina MiSeq sequencing. The presence of microorganisms of all metabolic groups illustrates the importance of a balanced and diverse biomass to have a robust and stable methanogenic reactor. The relative abundance of families of proteolytic bacteria was high, while hydrogenotrophic methanogens, such as Methanobacteriaceae, dominated the methanogenic community in the reactor. The putative methanogenic lineage of unassigned WCHA1-57 became dominant at the end of the experiment, but it was unassigned Woesearchaeota that dominated the archaeal community for most part of the experiment. An interaction between proteolysis, amino acid degradation and syntrophic methanogenesis was proposed among members of Porphyromonadaceae, unassigned Cloacimonetes and methanogenic archaea.
\end{abstract}

Keywords: Anaerobic digestion, microbial community, volumetric loading rate disturbance, hydrogen concentration. 


\section{Introduction}

Anaerobic membrane bioreactors (AnMBR) have emerged in the last decades as one of the innovative options to treat wastewaters from food processing, paper, pharmaceutical, landfill and textile industries (Aquino et al., 2006; Yang et al., 2006; Le-Clech, 2010). The reported advantages of AnMBR over conventional wastewater treatment systems are: high biomass retention, excellent effluent quality, low sludge production, a small footprint and high net energy production (Liao et al., 2006; Wan et al., 2011). Although biofouling (He et al., 2005; Dereli et al., 2014) and disruption of flocs (Stroot et al., 2001; Padmasiri et al., 2007) are some of the main challenges in the application of AnMBR, other factors also affect the treatment performance and stability of the reactor. These include operational conditions such as temperature, hydraulic retention time (HRT) and organic loading rate (OLR) (Skouteris et al., 2012; Lin et al., 2013; Ozgun et al., 2013).

The sludge composition, or more specifically the structure and functionality of the microbial communities in the bioreactor are essential for good performance of anaerobic digestion (AD) (Carballa et al., 2015; Lucas et al., 2015). Methanogenic communities of bacteria and archaea play an indispensable role in the complete conversion of organic material to methane and carbon dioxide (Stams et al., 2012). An imbalance among acidogenesis, fermentation and methanogenesis will generally lead to accumulation of volatile fatty acids (VFA) and in some cases to a complete collapse of the system. Ammonia inhibition, acidification, and foaming, especially at high organic loading rates are causing these instabilities (Guo et al., 2014; Li et al., 2015). Several studies have addressed the link between OLR disturbances and the microbial communities (Belostotskiy et al., 2015; Li et al., 2016). Exploration of the microbial community composition in anaerobic digesters is important as it increases our understanding of the different processes occurring in the reactor.

Pot ale is the main liquid by-product of whiskey distilleries (Melamane et al., 2007; Graham et al., 2012). Large volumes of pot ale are generated during production of whiskey; for every litre of alcohol, 8 - 15 litres of pot ale are produced (Mohana et al., 2009). Distillery spent wash is considered a high-strength wastewater with a very high chemical and biochemical oxygen demand (COD and BOD) and with low $\mathrm{pH}$ (Acharya et al., 2008). These characteristics and the large quantities of pot ale generated annually, have attracted interest in using this by-product for biogas production in anaerobic digesters (Tokuda et al., 1998; Goodwin et al., 2001; Barrena et al., 2017).

The particular composition of pot ale rich in protein, phosphorous and organic acids requires a robust system that can endure the variations in the composition of the feed, as well as the high levels of VFA and ammonia, without limiting the biogas production in the system. Ammonia, above certain concentration thresholds, can 
have an inhibitory effect on methanogens (Vidal et al., 2000; Ariunbaatar et al., 2015). Ammonia levels between 50 and $200 \mathrm{mgll}^{-1}$ stimulate methanogens (McCarthy, 1964), but free ammonia concentrations higher than $300 \mathrm{mg} \mathrm{l}^{-1}$ have shown severe anaerobic treatment inhibition in mesophilic conditions, presumably by inhibiting the methanogens (Omil, 1995). Ammonia toxicity levels are highly dependent on $\mathrm{pH}$ and temperature since the cause of toxicity is the un-ionized form of ammonia (free ammonia) which is the dominant form at high $\mathrm{pH}$ and temperatures (Vidal et al., 2000; Rajagopal et al., 2013; Yenigun and Demirel, 2013). Several studies indicate that hydrogenotrophic methanogens (from the orders Methanomicrobiales, Methanococcales, Methanocellales, Methanobacteriales and Methanopyrales) are more tolerant to the ammonia toxicity than acetoclastic methanogens (from the orders Methanosarcinaceae and Methanosaetaceae), regardless the temperature of the experimental conditions (Koster and Lettinga, 1984; Angelidaki and Ahring, 1993; Calli et al., 2005; Zhang et al., 2014; Wang et al., 2016).

The inhibitory effect of VFA on hydrolysis and methanogenesis is difficult to evaluate due to interactions between VFA concentrations and pH (Azman et al., 2015). High VFA concentrations can lead to a drop in $\mathrm{pH}$ values, and eventual toxic conditions in bioreactors. However, in highly buffered systems, the $\mathrm{pH}$ changes can be small, therefore, independently from $\mathrm{pH}, \mathrm{VFA}$ are usually tracked for process monitoring (Murto et al., 2004; Siegert and Banks, 2005). Researchers are not in agreement over which VFA is the best indicator for impending reactor failure: acetic acid, propionic acid, i-butyric, i-valeric or the ratio of propionic to acetic acid (Marchaim and Krause, 1993; Boe et al., 2008; Franke-Whittle et al., 2014).

Besides ammonia and VFA, other compounds which are known to cause toxic or have inhibitory effects in biogas reactors and affect methanogenesis are hydrogen sulfide, as well as hydrogen and heavy metals (Guwy et al., 1997; Franke-Whittle et al., 2014; Paulo et al., 2015). Many hydrogen-producing reactions are thermodynamically unfavourable unless the partial pressure of hydrogen is kept low (Thauer et al., 1977; Schink, 1997; Kleerebezem and Stams, 2000). Therefore, hydrogen consuming methanogens play an indispensable role in maintaining hydrogen levels low in syntrophic communities with bacteria (Stams et al., 2012). Thus, an increase in hydrogen might be useful to predict disturbances between fermentative processes and methanogenesis (Conrad, 1999; Junicke et al., 2015). The use of hydrogen concentrations as a performance monitor for anaerobic digestion has been addressed before with inconsistent conclusions (Mosey and Fernandes, 1989; Kidby and Nedwell, 1991; Cord-Ruwisch et al., 1997).

This study analysed microbial diversity, process parameters and performance during the start-up and stable operation of a mesophilic AnMBR treating pot ale, as well as the robustness of the bioreactor and the stability of biomass composition to 
overloading events. Moreover, we investigated if hydrogen concentrations can be used as an early warning indicator of process instability.

\section{Materials and methods}

\section{Operation of the membrane bioreactor}

A pilot-scale anaerobic membrane bioreactor with a working liquid volume of 10 litres was continuously fed and operated for 242 days. The continuous flow stirredtank reactor (CSTR) was inoculated with crushed and sieved granular anaerobic

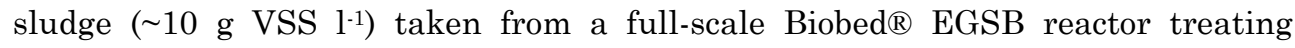
fermentation industry wastewater. 1 litre of sludge was added to the reactor and mixed with $5 \mathrm{~g}^{\mathrm{l}^{-1}} \mathrm{NaHCO}_{3}$ up to the final working volume. The reactor was fed with pot ale and maintained at a constant temperature of $37^{\circ} \mathrm{C}$. The $\mathrm{pH}$ was kept at 7.3 \pm 0.2 by dosing $2 \mathrm{M} \mathrm{NaOH}$ when necessary (Supporting information, Fig. S6.1).

The feed, obtained from a whiskey distillery in Scotland, was kept at a controlled temperature of $4^{\circ} \mathrm{C}$ and magnetic stirring before entering the CSTR. The composition of the feed is listed in Supporting information, Table S6.1. Undiluted pot ale was fed for the first 120 days; after that, the feeding continued with tap water diluted pot ale $(1: 1 \mathrm{vol} / \mathrm{vol})$ until the end of the experiment.

An ultrafiltration cross flow module composed of a tubular polyvinylidene fluoride membrane ( $80 \mathrm{~cm}$ length, $5.2 \mathrm{~mm}$ inner diameter and $30 \mathrm{~nm}$ pore size) separated the biomass from the effluent. Volatile fatty acids (VFA) in the permeate were quantified daily using a Varian 3900 gas chromatograph (Agilent, Santa Clara, CA) equipped with a silica column (25 $\mathrm{m}$ and $0.53 \mathrm{~mm}$ internal diameter) and a flame ionization detector. Samples of feed and sludge were centrifuged ( $5 \mathrm{~min}, 10,000 \mathrm{x} \mathrm{g}$ ) and passed through $0.45 \mu \mathrm{m}$ cellulose filters prior to their quantification.

The biogas production of the CSTR was measured with a wet tipping biogas meter and its composition was monitored continuously via the online Agilent 490 micro gas chromatograph (Agilent, Santa Clara, CA) equipped with two channels (Molsieve 5A column for $\mathrm{H}_{2}$; PPQ $10 \mathrm{~m}$ column for $\mathrm{CH}_{4}$ and $\mathrm{CO}_{2}$; thermal conductivity detector; Argon and Helium were used as carrier gases).

Volumetric loading rates (VLR) varied during the experiment. By gradually increasing the feed flow during the start-up stage of the reactor an optimum VLR was established at $5 \pm 0.8 \mathrm{~g} \cdot \mathrm{COD}^{\mathrm{l}} \mathrm{l}^{-1} \cdot \mathrm{day}^{-1}$ after day 140 . The hydraulic retention time (HRT) was kept at 10 days. Table 6.1 summarizes the operational conditions of the reactor, the different stages of functioning and the stages when the high load tests took place. 
High load tests (HLT) were applied to the reactor by increasing the VLR for short defined periods of time. The first overload test was done at day 149 in which the VLR was increased from 4 to $10 \mathrm{~g} \cdot \mathrm{COD} \cdot \mathrm{r}^{-1} \cdot \mathrm{day}^{-1}$ for 4 hours. An increase up to $11 \mathrm{~g} \cdot \mathrm{COD} \cdot \mathrm{l}$ ${ }^{1}$ day $^{-1}$ of the VLR was applied for the second HLT for 6 hours on day 156. The third high load test (day 184) increased the VLR levels from 6 to 9.5 and then up to 12.5 $\mathrm{g} \cdot \mathrm{COD}^{\mathrm{l}^{-1}}$ day $^{-1}$ over a period of 6 hours. During the HLT experiments, $\mathrm{H}_{2}$ concentrations of the biogas were measured online with the gas chromatographer every 12 minutes. Permeate samples for VFA analysis were collected every 30 minutes.

Multiple physicochemical and biological parameters were regularly analysed in the feed, sludge and permeate to monitor the operational status of the AnMBR (TCOD, T(S)S, V(S)S, TKN, SKN, Total-P, ortho-P, VFA, anions, cations, etc.). Moreover, sludge samples were taken along the different stages of operation to perform chemical and microbiological analysis. For the $1^{\text {st }}$ HLT, the sludge was extracted from the reactor one day before the experiments (day 148). This was done to allow the pressure on the AnMBR to stabilize and have an $\mathrm{H}_{2}$ baseline measurement with the GC as constant as possible before the increase in the VLR. Subsequent sludge samples were taken a day after the $1^{\text {st }}$ HLT (day 150) and after the $3^{\text {rd }}$ HLT (day 184).

Specific acetoclastic methanogenic activity (SAMA) assays were carried on using Oxitop ${ }^{\circledR}$ equipment (WTW, Weilheim, Germany). $300 \mathrm{ml}$ bottles were filled with 49.4 $\mathrm{ml}$ of sludge from the reactor and mixed with $0.6 \mathrm{ml}$ sodium acetate $\left(250 \mathrm{~g} \cdot \mathrm{COD} \cdot \mathrm{r}^{-1}\right)$. The initial F/M-ratio was $0.17 \mathrm{~g} \cdot \mathrm{COD} \cdot \mathrm{g} \cdot \mathrm{VSS}^{-1}$. Anaerobic conditions were provided by flushing bottles with $\mathrm{N}_{2} / \mathrm{CO}_{2}$ mixture (70:30, vol/vol). The bottles were placed in shakers at $37{ }^{\circ} \mathrm{C}$. Biogas and methane production was calculated by measuring the pressure increase in the bottles every 20 minutes by Oxitop ${ }^{\circledR}$ heads. The analyses were performed in duplicates and two consecutive feeds with sodium acetate were applied in each replicate.

Total Kjeldahl Nitrogen (TKN) was determined with Simplified TKN Kit ${ }^{\text {TM }}$ (Hach Company, Loveland, CO). COD was measured with Hach Lange Kits ${ }^{\text {TM }}$ (Hach Company, Loveland, CO). 


\begin{tabular}{|c|c|c|c|c|}
\hline \multicolumn{5}{|c|}{$\begin{array}{l}\text { Table 6.1. Operational conditions for } 242 \text { days of an anaerobic membrane } \\
\text { bioreactor operated at } 37^{\circ} \mathrm{C} \text { and HRT of } 10 \text { days. }\end{array}$} \\
\hline Stage & $\begin{array}{c}\text { Operation } \\
\text { time(days) }\end{array}$ & $\begin{array}{c}\text { Feed } \\
\text { details }\end{array}$ & $\begin{array}{c}\text { VLR } \\
\left(\mathrm{g} \cdot \mathrm{COD}^{\cdot} \cdot \mathbf{l}^{-1} \cdot \mathrm{d}^{-1}\right)\end{array}$ & Notes \\
\hline 0 & 1 & \multirow[b]{2}{*}{$\begin{array}{l}\text { Undiluted } \\
\text { Pot Ale }\end{array}$} & 1.2 & Inoculation \\
\hline $\begin{array}{c}\text { I } \\
\text { Start-up }\end{array}$ & $2-120$ & & $1.2-8.5$ & $\begin{array}{l}\text { Unstable performance } \\
\text { characterised by } \\
\text { permeate }\end{array}$ \\
\hline $\begin{array}{l}\text { II } \\
\text { Detox }\end{array}$ & $121-140$ & $\begin{array}{c}\text { Tap water } \\
\text { Diluted Pot } \\
\text { Ale (1:1) \& } \\
\mathrm{NaHCO}_{3} \\
\end{array}$ & $3-5.4$ & $\begin{array}{l}\text { Accumulated VFA after } \\
\text { ammonia inhibition were } \\
\text { flushed away }\end{array}$ \\
\hline $\begin{array}{l}\text { III } \\
\text { Stable operation }\end{array}$ & $141-148$ & \multirow{8}{*}{$\begin{array}{c}\text { Tap water } \\
\text { Diluted Pot } \\
\text { Ale (1:1 vol. } \\
\text { /vol.) }\end{array}$} & $3.8-5$ & $\begin{array}{l}\text { VFA in the permeate: } \\
\quad<5 \text { meq } \mathrm{l}^{-1}\end{array}$ \\
\hline IV & $149-15$ & & 10 & \multirow{6}{*}{$\begin{array}{l}\text { High Load Tests (HLT) } \\
\text { and recovery of the } \\
\text { bioreactor }\end{array}$} \\
\hline $1^{\text {st }} \mathrm{HLT}$ & $149-155$ & & 4.1 & \\
\hline $\mathrm{V}$ & & & 11 & \\
\hline $2^{\text {nd }} H L T$ & $156-183$ & & $4.1-6.1$ & \\
\hline \multirow{2}{*}{$\begin{array}{c}\mathrm{VI} \\
3^{\text {rd }} \text { HLT }\end{array}$} & \multirow{2}{*}{$184-218$} & & 12.5 & \\
\hline & & & $4.8-5.2$ & \\
\hline $\begin{array}{c}\text { VII } \\
\text { Stable operation }\end{array}$ & 219- 242 & & $5.5-8.4$ & Stable performance \\
\hline
\end{tabular}

\section{Microbial community analyses by next generation $16 \mathrm{~S}$ rRNA amplicon sequencing}

Sludge samples $(\sim 50 \mathrm{ml})$ were collected during 16 sampling points (Figure 6.1). Samples were kept at $-80^{\circ} \mathrm{C}$ prior to genomic DNA extraction. Samples were washed with PBS solution with $0.5 \mathrm{mM}$ EDTA twice to remove humic acids and other contaminants that could have an inhibitory effect on DNA extraction and PCR reactions. Genomic DNA was extracted from the sixteen samples with technical duplicates using the FastDNA® Spin Kit for Soil (MP Biomedicals, Santa Ana, CA) according to the manufacturer's protocol with additional two 45-second beat beating steps with a FastPrep Instrument (MP Biomedicals). Concentrations and quality of the obtained DNA were determined with a Nanodrop ${ }^{\circledR}$ (ND-1000) spectrophotometer (Nanodrop Technologies, Wilmington, DE). Extracted DNA from selected samples was used for bacterial and archaeal community analyses. The amplification of bacterial and archaeal 16S rRNA gene fragments was done using a 2-step PCR protocol. The first PCR amplification of bacterial 16S rRNA gene fragments was done using a set of primers composed by the 27F-Degs (5'GTT[TC]GAT[TC][AC]TGGCTCAG-3') (Van den Bogert et al., 2011; van den Bogert et al., 2013) and an equimolar mix of two reverse primers; 338R-I and 338-R-II (5'- 
GC[AT]GCC[AT]CCCGTAGG[TA]GT-3') (Daims et al., 1999). For the archaeal 16S rRNA gene amplification primers 518F (5'-CAGC[AC]GCCGCGGTAA-3') (Wang and Qian, 2009) and 905R (5'-CCCGCCAATTCCTTTAAGTTTC-3') (Kvist et al., 2007) were used. PCR amplifications were carried out using $500 \mathrm{nM}$ of each forward and reverse primer (Biolegio BV, Nijmegen, The Netherlands), 1 unit of Phusion Hot Start DNA polymerase (Thermo Scientific, USA), $10 \mu \mathrm{l}$ of 5x HF-buffer, $200 \mu \mathrm{M}$ dNTP mix and $1 \mu \mathrm{l}$ DNA template. PCR grade water was used to fill up the reaction mixture to $50 \mu \mathrm{l}$. The PCR amplification proceeded as follows: a pre-denaturing step of $99{ }^{\circ} \mathrm{C}$ for $30 \mathrm{~s}, 25$ cycles of denaturing at $98{ }^{\circ} \mathrm{C}(10 \mathrm{~s})$, annealing at $56{ }^{\circ} \mathrm{C}$ for bacterial and $60^{\circ} \mathrm{C}$ for archaeal $(20 \mathrm{~s})$, extension at $72^{\circ} \mathrm{C}(20 \mathrm{~s})$ and a final extension at $72^{\circ} \mathrm{C}(10 \mathrm{~min})$. After positive amplifications, technical duplicates were pooled and prepared for the second PCR amplification. This second step was performed to extend $8 \mathrm{nt}$ barcodes to the amplicons, as described previously (Hamady et al., 2008). Barcoded amplification was performed using $5 \mu \mathrm{l}$ of the first PCR product, $500 \mathrm{nM}$ of each forward and reverse primer (Biolegio BV, Nijmegen, The Netherlands), 2 units of Phusion Hot Start DNA polymerase (Thermo Scientific, Waltham, MA), $20 \mu \mathrm{l}$ of 5x HF-buffer, $200 \mu \mathrm{M}$ dNTP mix, and filling up the reaction mixture to $100 \mu \mathrm{l}$ with nuclease free water. The second PCR program was as follows: the $98{ }^{\circ} \mathrm{C}$ predenaturing step for $30 \mathrm{~s}$, five cycles of $98^{\circ} \mathrm{C}(10 \mathrm{~s}), 52^{\circ} \mathrm{C}(20 \mathrm{~s}), 72^{\circ} \mathrm{C}(20 \mathrm{~s})$ and 72 ${ }^{\circ} \mathrm{C}$ (10 min). Barcoded PCR products were cleaned using the HighPrep ${ }^{\mathrm{TM}}$ PCR cleanup system (MagBio Genomics Inc., Gaithersburg, MD). DNA was quantified using Qubit® 2.0 Fluorometer (Thermo Fisher Scientific, MA). After the second PCR, barcoded samples were pooled in equimolar quantities to create a library. Archaeal and bacterial libraries were purified a second time and sent to GATC Biotech (Konstanz, Germany) for sequencing on the Illumina MiSeq platform.

\section{Sequencing data analysis}

16S rRNA gene sequencing data was analysed using NG-Tax, an in-house pipeline (Ramiro-Garcia et al., 2016). Paired-end libraries were filtered to contain only read pairs with perfectly matching barcodes, and those barcodes were used to demultiplex reads by sample. Resulting reads were separated by sample using the affiliated barcodes. Taxonomy affiliation was done with the SILVA 16S rRNA reference database by using an open reference approach as described by (Quast et al., 2013). The minimum threshold that an operational taxonomic unit (OTU) needs to be present compared to the whole database, the percentage identity threshold for the blastn and the percentage for the error correction were settled in default values, 0.1 , 100 and 98.5, respectively. Quantitative Insights into Microbial Ecology (QIIME) v1.2 (Caporaso et al., 2010) was used to define microbial compositions based on the described pipeline.

Filtering, subsetting and diversity analyses of the microbial communities were performed using the phyloseq $\mathrm{R}$ package for reproducible interactive analysis and 
graphics of microbiome census data (McMurdie and Holmes, 2013). All non-bacterial and unassigned OTUs were removed from the bacterial samples, as well as all nonarchaeal and unassigned OTUs were removed from the archaeal samples prior to any downstream analysis. Samples with $<2,500$ reads were also removed. The read counts of the merged technical replicates were normalized using the CSS method from metagenomeSeq package v1.11 (Paulson et al., 2013) in R v.3.3.2 (Team, 2016).

\section{Results and discussion}

\section{Start-up period of the reactor}

During the start-up period of the reactor, diverse operational problems such as membrane blockage and fouling, gas leakage and pump failures occurred throughout the first 60 days of operation. In addition, change in composition of the pot ale, supplied by the whiskey distillery, caused problems during the start-up period. A gradual increase in the Total Kjeldahl Nitrogen (TKN) levels in the pot ale from 1.5 $\mathrm{g} \cdot \mathrm{l}^{-1}$ at the beginning of the operation of the reactor up to $4.9 \mathrm{~g} \mathrm{l}^{-1}$, around day 110 to 120, resulted in VFA accumulation to levels above $20 \mathrm{meq}^{-1}$ (Supporting information, Fig. S2).

The operational variations in the distillery industry results in a highly variable composition of pot ale. High levels of protein, lactic acid and yeast cells that sink in the bottom of the whiskey fermenters are commonly reported in pot ale studies (Goodwin and Stuart, 1994; Graham et al., 2012; Barrena et al., 2017). Protein breakdown occurs during anaerobic digestion which leads to the build-up of ammonia. Therefore, as the levels of TKN in the feed and in the reactor increased, ammonia inhibition of methanogens might have occurred in the AnMBR.

When hydrogen stops being consumed by methanogens it accumulates and syntrophic interactions are disrupted, but also some non-syntrophic fermentative reactions cannot occur anymore, such as lactate and ethanol conversion to acetate (Giovannini et al., 2016). The increase in $\mathrm{H}_{2}$ levels (above $100 \mathrm{ppm}$ ) and VFA accumulation (mainly composed of acetate) observed between day 116 and 136 are an indication of a disruption between hydrogen-producing and hydrogenotrophic microbial communities, most probably caused by the high levels of ammonia in the reactor. Figure 6.1 shows the reactor performance along the 242 days of operation. Repeated VFA accumulation peaks can be noticed at the beginning of the start-up period and principally when the reactor was affected by ammonia toxicity. 


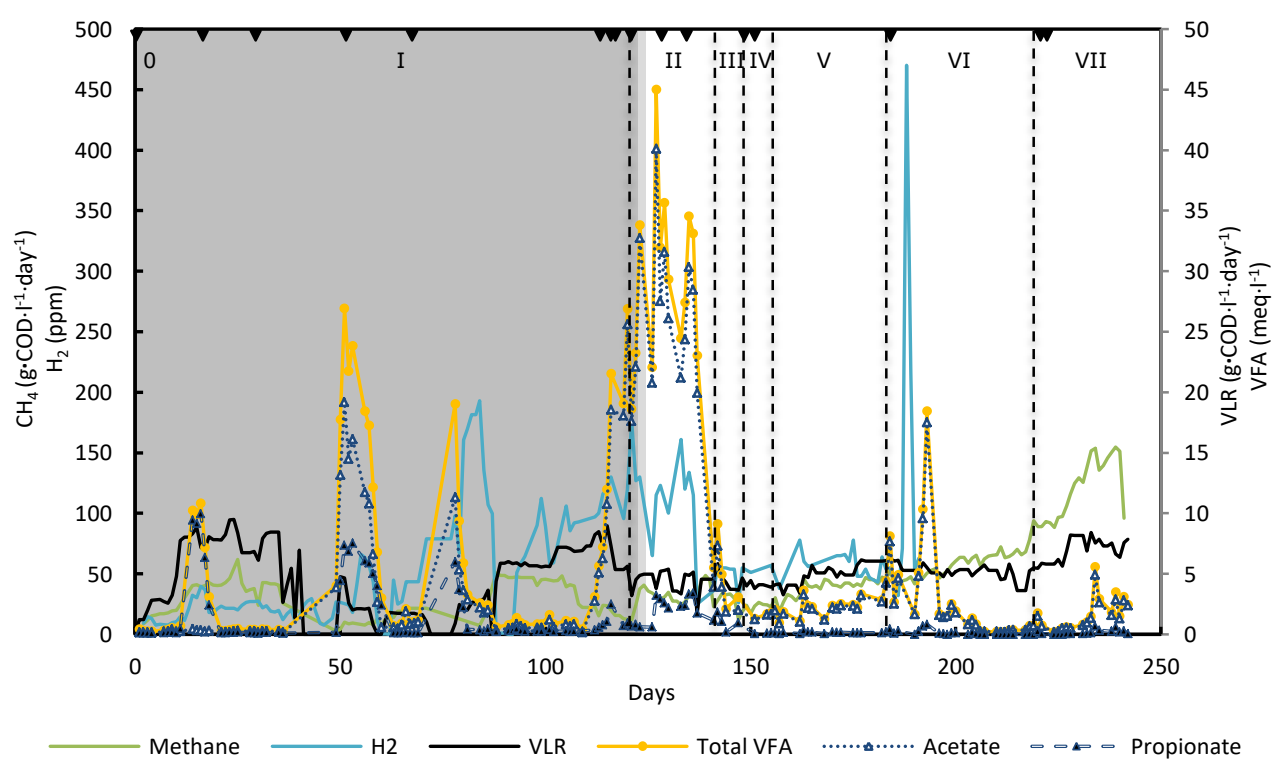

Figure 6.1. Performance of the AnMBR operated at $37{ }^{\circ} \mathrm{C}$ for 242 days. Volumetric loading rate (VLR), total volatile fatty acids (VFA), acetate, propionate, methane and $\mathrm{H}_{2}$ are plotted. The dark area indicates undiluted and white area diluted pot ale dosing. Triangles marks $(\boldsymbol{\nabla})$ at the top of the plot show the sampling points for the microbiological analyses. Reactor stages are divided by dotted lines and signalled with roman numbers.

It has been reported that total ammonia (TAN) and free ammonia (FAN) concentrations above $3 \mathrm{~g} \cdot \mathrm{NH}_{4}{ }^{+}-\mathrm{N} \cdot \mathrm{l}^{-1}$ and $0.15 \mathrm{~g} \cdot \mathrm{NH}_{3}-\mathrm{N} \cdot \mathrm{l}^{-1}$, respectively, have an obvious inhibitory effect on methanogenesis and lower the potential methane yield in anaerobic digesters (Wang et al., 2015; Tian et al., 2017). A study has shown that syntrophic acetate oxidation coupled with hydrogenotrophic methanogenesis is the dominant pathway in digesters with high ammonia levels $\left(2.8-4.57 \mathrm{~g} \cdot \mathrm{NH}_{4}{ }^{+} \mathrm{N} \cdot \mathrm{l}^{-1}\right)$, while acetoclastic methanogenesis dominate at low ammonia $\left(<1.21 \mathrm{~g} \cdot \mathrm{NH}_{4}{ }^{+}-\mathrm{N}^{-1}\right)$ (Fotidis et al., 2013).

To reverse the ammonia inhibitory effect on methanogens, the pot ale was diluted with tap water to maintain the TKN levels lower than $2.5 \mathrm{~g} \cdot \mathrm{l}^{-1}$. Additionally, the bioreactor was fed with a $\mathrm{NaHCO}_{3}$ solution on day 137 to flush away the accumulated VFA. These actions were effective, and a stable performance of the reactor was finally achieved with VFA levels $<5$ meq $\mathrm{l}^{-1}$ in the permeate and $\sim 50 \mathrm{ppm}$ of $\mathrm{H}_{2}$ in the biogas at day 140. Once the AnMBR was stable a maximum capacity of the reactor was settled at $\sim 5 \mathrm{~g} \cdot \mathrm{COD}^{\mathrm{l}} \mathrm{l}^{-1} \cdot \mathrm{day}^{-1}$. 


\section{High load tests period}

Once the pot ale fed to the reactor was diluted and the reactor stabilized, overloading experiments were carried out to assess the robustness and evaluate the response of the reactor to drastic increases of VLR. In Figure 6.2 we can observe in detail the $\mathrm{H}_{2}$ and VFA responses to the high load tests. The first high load test (HLT) was done at day 149 when the system was performing stable at an aimed VLR of $4 \mathrm{~g}$ COD $\mathrm{l}$ ${ }^{1}$ day $^{-1}$. For four hours the VLR was increased to $10 \mathrm{~g} \cdot \mathrm{COD}^{-1} \cdot \mathrm{day}^{-1}$. Total-VFA and $\mathrm{H}_{2}$ levels increased in response to this overloading event. Four hours after, when the load was set back to $4 \mathrm{~g} \cdot \mathrm{COD}^{-1} \mathrm{~d}^{-1} \mathrm{day}^{-1}$, the $\mathrm{H}_{2}$ concentration decreased to normal levels of $\sim 55 \mathrm{ppm}$ rapidly after it reached a maximum of $70.5 \mathrm{ppm}$. Total-VFA concentrations of the permeate increased from 1.6 to 5.7 meq $\mathrm{l}^{-1}$ and took longer to decrease to normal levels of $<1.5 \mathrm{meq}^{\mathrm{l}-1}$. Acetate was the main component in the increase of Total-VFA.
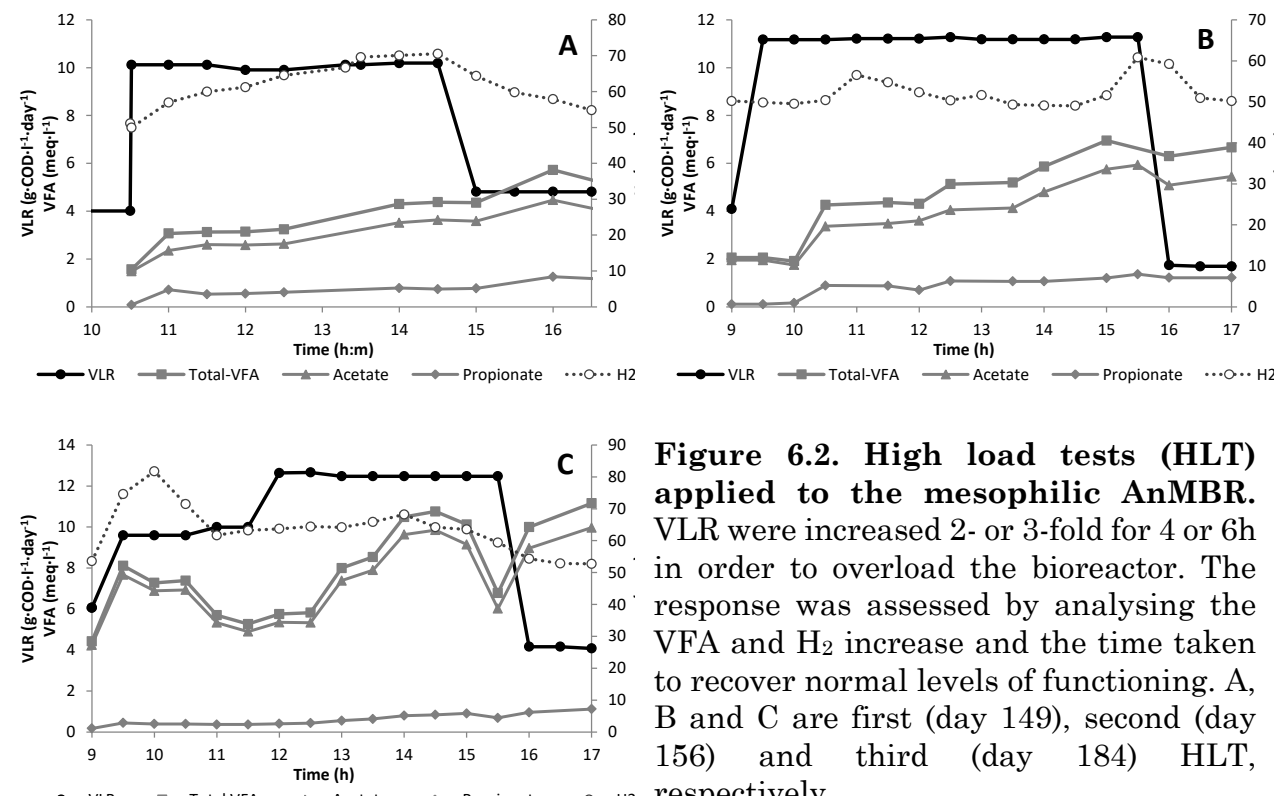

Figure 6.2. High load tests (HLT) applied to the mesophilic AnMBR. VLR were increased 2- or 3-fold for 4 or $6 \mathrm{~h}$ in order to overload the bioreactor. The response was assessed by analysing the VFA and $\mathrm{H}_{2}$ increase and the time taken to recover normal levels of functioning. A, $\mathrm{B}$ and $\mathrm{C}$ are first (day 149), second (day 156) and third (day 184) HLT, respectively.

The effect of the first high load test in the performance of the bioreactor was mild. No significant differences were found in the specific acetoclastic methanogenic activities before and after the HLT (Table 6.2). A second HLT was done by increasing the VLR further, up to $11 \mathrm{~g} \cdot \mathrm{COD}^{\mathrm{r}} \mathrm{l}^{-1} \mathrm{day}^{-1}$ on day 156 for a longer period (6 hours). This time, the $\mathrm{H}_{2}$ levels only reached a maximum of $60.9 \mathrm{ppm}$ and rapidly recovered when the VLR was decreased. Total-VFA reached $7 \mathrm{meq}^{\mathrm{l}-1}$, although the initial values were 2.1 meq $\mathrm{l}^{-1}$. The main accumulated fatty acid was acetate up to $5.9 \mathrm{meq} \cdot \mathrm{l}$ ${ }^{1}$, whereas propionate increased only to $1.4 \mathrm{meq} \cdot \mathrm{l}^{-1}$. 
As the reactor proved to be robust to the short term HLT, the potential of the reactor to operate in a higher VLR for a longer period was explored after day 160. Starting with $4 \mathrm{~g} \cdot \mathrm{COD}^{\mathrm{l}} \mathrm{l}^{-1} \cdot \mathrm{day}^{-1}$ the VLR was gradually increased to $6 \mathrm{~g} \cdot \mathrm{COD}^{\mathrm{l}-1} \cdot \mathrm{day}^{-1}$ in a period of 10 days (days 160-170).

On day 184, a third HLT was carried for 6 hours with an escalated VLR increase (Figure 6.2.C). After an hour of the HLT, $\mathrm{H}_{2}$ concentration reached $81.7 \mathrm{ppm}$. However, it decreased to levels of $\sim 68 \mathrm{ppm}$ as in the previous HLTs. Total-VFA accumulation, mainly composed of acetate, reached higher levels $\left(11.2 \mathrm{meq}^{\mathrm{l}-1}\right)$ as the load test was higher this time.

Although the reactor was normally recovering to the last HLT and the $\mathrm{H}_{2}$ and VFA levels were dropping back to normal, another setback occurred when pot ale had to be replaced with vinasse due to issues with the delivery of pot ale from the distillery in Scotland. Vinasse was used to feed the reactor for 4 days (days 186-190). Vinasse had also been used in day 77 due to lack of pot ale, but only for a day. The change of feed resulted in VFA accumulation up to 18.5 meq $\mathrm{l}^{-1}$ and a peak on $\mathrm{H}_{2}$ concentration up to $470 \mathrm{ppm}$. The reactor stopped, and it was restarted with pot ale as feed and a VLR of $5 \mathrm{~g} \cdot \mathrm{COD}^{-1} \mathrm{~d}^{-1} \mathrm{day}^{-1}$. During the last month of the experiment, VLR was gradually increased to $8 \mathrm{~g} \cdot \mathrm{COD}^{-1} \mathrm{l}^{-1}$ day $^{-1}$, while the VFA remained at low acceptable levels $\left(2.5-3 \mathrm{meq}^{-1}\right)$. The reactor proved to have the capacity to recover despite these different disturbances.

\section{Performance of the reactor}

The specific acetoclastic methanogenic activity was tested on sludge samples from the bioreactor one day before and one or two days after the HLTs (Table 6.2). No significant differences were found between the measurements before and after HLTs, and obtained values fell within normal range for anaerobic sludge $(0.1-1 \mathrm{~g} \cdot \mathrm{COD} \cdot \mathrm{g}$ $1 \cdot$ VSS $\left.\cdot d^{-1}\right)$ (Fang et al., 1994; Regueiro et al., 2012). The performance of the AnMBR resulted in a COD removal of $97.1 \%( \pm 2.4 \%)$, with a biogas production rate of $27 \pm 3$ $\mathrm{l} \cdot \mathrm{d}^{-1}$, composed of $60 \% \mathrm{CH}_{4}( \pm 3.1 \%)$.

\begin{tabular}{|c|c|c|c|c|}
\hline \multirow{2}{*}{ HLT } & \multicolumn{2}{|c|}{ SAMA Test Before HLT* } & \multicolumn{2}{|c|}{ SAMA Test After HLT* } \\
\hline & $1^{\text {st }}$ feed & $2^{\text {nd }}$ feed & $1^{\text {st }}$ feed & $2^{\text {nd }}$ feed \\
\hline $\begin{array}{l}1^{\text {st }} \text { HLT (day } \\
149)\end{array}$ & $0.26( \pm 0.04)$ & $0.24( \pm 0.01)$ & $0.28( \pm 0.01)$ & $0.22( \pm 0.01)$ \\
\hline $\begin{array}{c}2^{\text {nd }} \text { HLT (day } \\
156)\end{array}$ & $0.27( \pm 0.01)$ & $0.23( \pm 0.01)$ & $0.31( \pm 0.05)$ & $0.26( \pm 0.01)$ \\
\hline $\begin{array}{c}3^{\text {rd }} \text { HLT (day } \\
184 \text { ) }\end{array}$ & $0.14( \pm 0.0)$ & $0.12( \pm 0.01)$ & $0.11( \pm 0.01)$ & $0.12( \pm 0.0)$ \\
\hline
\end{tabular}


Acetate and propionate were the main fatty acids accumulated when the reactor faced disturbances in its performance. Some studies report that acetic and butyric acid concentrations of 2,400 and $1,800 \mathrm{mg} \cdot \mathrm{l}^{-1}$, respectively, had no significant impact on the activity of methanogens while a propionic acid concentration of $900 \mathrm{mg} \cdot \mathrm{l}^{-1}$ resulted in inhibition of methanogens and decreased bacterial numbers(Wang et al., 2009). Others proposed that acetic acid levels higher than $800 \mathrm{mg} \cdot \mathrm{l}^{-1}$ or a propionic to acetic acid ratio larger than 1.4 predict digester failure (Buyukkamaci and Filibeli, 2004; Romsaiyud et al., 2009).

During the start-up stage of our reactor, three peaks of total-VFA exceeding $800 \mathrm{mg} \cdot \mathrm{l}$ ${ }^{1}$ could be seen when the reactor was fed with undiluted pot ale (day 16, 50-57 and 78) (Figure 6.1). For the first of these peaks propionate was the main component with a propionic to acetic acid ratio of 37 . For the other two peaks propionate still contributed to $>35 \%$ of the total-VFA, however more acetate $\left(1,150 \mathrm{mg} \cdot \mathrm{l}^{-1}\right.$ on day 51 and $680 \mathrm{mg} \mathrm{l}^{-1}$ on day 78) than propionate (543 $\mathrm{mg}^{\mathrm{l}^{-1}}$ on day 51 and $444 \mathrm{mg}^{\mathrm{l}^{-1}}$ on day 78) was accumulated. Similarly, acetate was the main VFA accumulated during the period with ammonia inhibition (days 115-139), and after the bioreactor was fed with diluted pot ale, while the propionate share remained $\sim 10 \%$ of the total-VFA. In digesters treating high-strength waste with high ammonia content, ammonia inhibition will be the primary process controlling factor, but different systems will have their own levels of VFAs that can be considered 'normal' for the reactor (Angelidaki et al., 1993). Thus, it is not possible to indicate the state of an anaerobic process based only on VFA levels.

During the provoked disturbances in our AnMBR via the HLT, but also in the period with high ammonia concentrations and during the changes of feed to vinasse, the $\mathrm{H}_{2}$ concentrations varied significantly, in some cases after VFA accumulation events (day 81), in some other in parallel with the disturbance of the AnMBR (days 115-139) and in some others prior the collapse of the reactor (day 188) (Figure 6.1). In our study, the ease of on-line $\mathrm{H}_{2}$ concentration measurements and its rapid response to perturbations in the performance of the AnMBR supports the use of this parameter as an early warning indicator of process instability.

\section{Microbial community analysis}

The sequencing of the $16 \mathrm{~S}$ rRNA bacterial and archaeal genes resulted in an average of 300,108 reads for bacteria and 47,019 reads for the archaea. However, wrongly assigned OTUs were present at domain level. After filtering out the wrongly assigned OTUs, the number of reads per sample ranged from 60,914 to 561,911 for bacteria and 4,767 to 114,533 for archaea. These reads were assigned to eighteen bacterial phyla, thirty classes, twenty-seven orders and forty-four families. For the archaeal reads, four phyla, five classes, five orders and nine families were correctly assigned (Supplementary Table 6.3). 


\section{Bacterial community dynamics}

The relative abundances of the assigned OTUs at family level are presented in Figure 6.3. Families with at least $1 \%$ relative abundance in a sample are presented in the plot. In the inoculum Porphyromonadaceae (28\%), Syntrophaceae (26\%), Anaerolineaceae (15\%) and Bacteroidaceae (12\%) were the dominant families.

Members of the Porphyromonadaceae produce VFA from the degradation of complex carbohydrates, proteins and peptides (Ziganshin et al., 2011). For instance, Proteiniphilum acetatigenes (Chen and Dong, 2005), Petrimonas sulfuriphila (Grabowski et al., 2005), Paludibacter propionicigenes (Ueki et al., 2006), Proteiniphilum saccharofermentans, Petrimonas mucosa and Fermentimonas caenicola (Hahnke et al., 2015; Hahnke et al., 2016) are all acetate or/and propionate producing bacteria, many isolated from mesophilic laboratory-scale biogas reactors. Therefore, this bacterial family is predicted to be involved in hydrolysis and acidogenesis during $\mathrm{AD}$ of the pot ale. Porphyromonadaceae remained abundant for great part of the start-up stage, reaching up to $50 \%$ of relative abundance at day 66 . The thriving of this family fits with its description as proteolytic bacteria and members of this family might have taken advantage of the high availability of peptide constituents of the pot ale for acidogenesis. After the feed was changed to diluted pot ale (day 121) the relative abundance of this family gradually decreased to only $2 \%$. A decrease in the supply of proteinaceous compounds might have limited growth of this family; however, although diluted, pot ale is still high in protein, so other compounds might have had an influence. At the end of the experiment the relative abundance of Porphyromonadaceae increased again to 24\%, possibly promoted by the feeding of the reactor with vinasse at days 186-190.

The relative abundance of Syntrophaceae quickly dropped from $26 \%$ in the inoculum to levels less than $2 \%$ during the start-up stage and it was not detected when the reactor reached a stable performance at day 140. For Anaerolineaceae, the relative abundance gradually decreased during the start-up period to remain within $2-3 \%$ during the HLTs stage while Bacteroidaceae disappeared from the sludge after the first days of operation of the AnMBR.

For unassigned Bacteroidia and unassigned Bacteroidetes the relative abundance increased up to $23 \%$ and $11 \%$, respectively, during the stable performance of the bioreactor (days 134-150). According to (De Vrieze et al., 2015) Bacteroidetes might be dominant in digesters operating at mesophilic conditions and under low VFA levels. After the start-up stage of the bioreactor and once the VFA concentrations were stabilized to remain lower than $200 \mathrm{mg} \mathrm{l}^{-1}$, the relative abundance of the Bacteroidetes became significant (day 127). At the end of the experiment the relative abundance reached $46 \%$. 
The relative abundance of Comamonadaceae in the bioreactor during the start-up stage, and the period with high ammonia levels, reached $28 \%$ on day 150 . Members of the Comamonadaceae family are aerobic bacteria known for their denitrifying activity in aerobic treatment systems (Khan et al., 2002; Sadaie et al., 2007). Nevertheless, several species, such as Comamonas koreensis, have been characterized as facultatively anaerobic (Chang et al., 2002; Peng et al., 2013). A study in an alternating aerobic/anaerobic reactor indicates a role of this family in biological phosphorus and phosphate removal (Ge et al., 2015). The average nitrate measured in the pot ale fed to the reactor was only $15 \mathrm{mg} \mathrm{l}^{-1}$, while higher phosphorus concentrations were reported in the feed $\left(\mathrm{P}_{\text {total }} 603 \pm 74 \mathrm{mgll}^{-1}\right.$, Supporting information, Table S6.1). Lower concentrations of total phosphorus than in the feed were measured in the permeate, mainly in form of orthophosphate (Supporting information, Figure S6.2). Therefore, the function of Comamonadaceae in our AnMBR could be of a polyphosphate accumulating organism (PAO), but as more members of this family are being isolated and characterized, other biological roles should be keep in mind for consideration (Subhash et al., 2016; Xie et al., 2016).

The information available of the Lentimicrobiaceae family is limited to a recently isolated anaerobic bacterium Lentimicrobium saccharophilum (Sun et al., 2016). This bacterium was isolated from methanogenic granular sludge in a full-scale mesophilic UASB reactor treating high-strength starch-based organic wastewater. L. saccharophilum grows on a narrow range of carbohydrates and the major fermentative end products from glucose were acetate, malate, propionate, formate and hydrogen. Our results show an increase of the relative abundance of Lentimicrobiaceae during the start-up stage of the experiment and until the ammonia reached toxic levels. When diluted pot ale was fed into the reactor Lentimicrobiaceae disappeared from bacterial community.

The unassigned Cloacimonetes was enriched during the start-up stage and remained important throughout the different stages of the AnMBR. During the stable performance of the bioreactor it accounted for a $20 \%$ of relative abundance, and this percentage increased to $39 \%$ at day 184 after the HLTs. Previously known as WWE1 (Rinke et al., 2013), uncultured members of Cloacimonetes have been linked to interactions with syntrophic methanogenic consortia (Lykidis et al., 2011; Wu et al., 2013). The authors proposed a role for uncultivated Cloacimonetes taxa in additional syntrophic interactions beyond the standard $\mathrm{H}_{2}$-producing syntroph-methanogen partnership that may serve to improve community stability. Another study proposed that Candidatus Cloacamonas acidaminovorans, an amino acid fermenter, is a syntrophic bacterium present in many anaerobic digesters (Pelletier et al., 2008). Moreover, (Nobu et al., 2015) found out that Atribacteria and Cloacimonetes may perform syntrophic propionate metabolism in a methanogenic bioreactor and speculate that chaining syntrophic interactions (secondary syntrophy) and substrate exchange may facilitate proteinaceous detritus metabolism. 


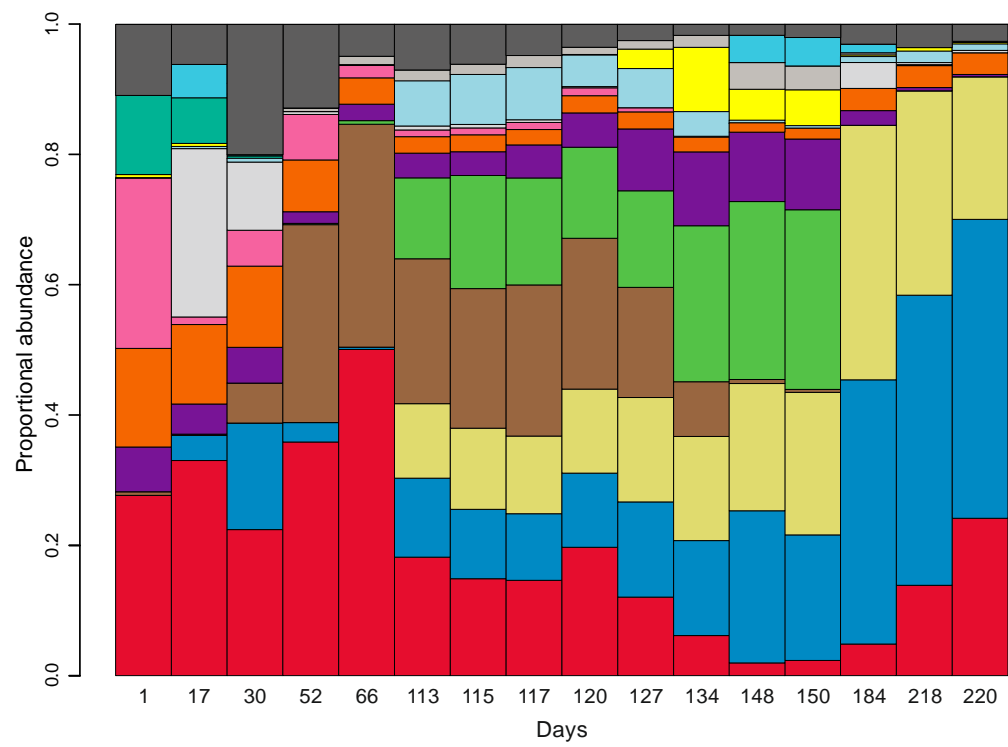

Bacterial Family

- Porphyromonadaceae

- Unassigned Bacteroidia

- Unassigned Cloacimonetes

- Lentimicrobiaceae

- Comamonadaceae

- Unassigned Bacteroidetes

- Anaerolineaceae

- Syntrophaceae

- Unassigned Elusimicrobia

- Unassigned OPB35

Ruminococcaceae

- Rikenellaceae

- Bacteroidaceae

n Prevotellaceae

- Other

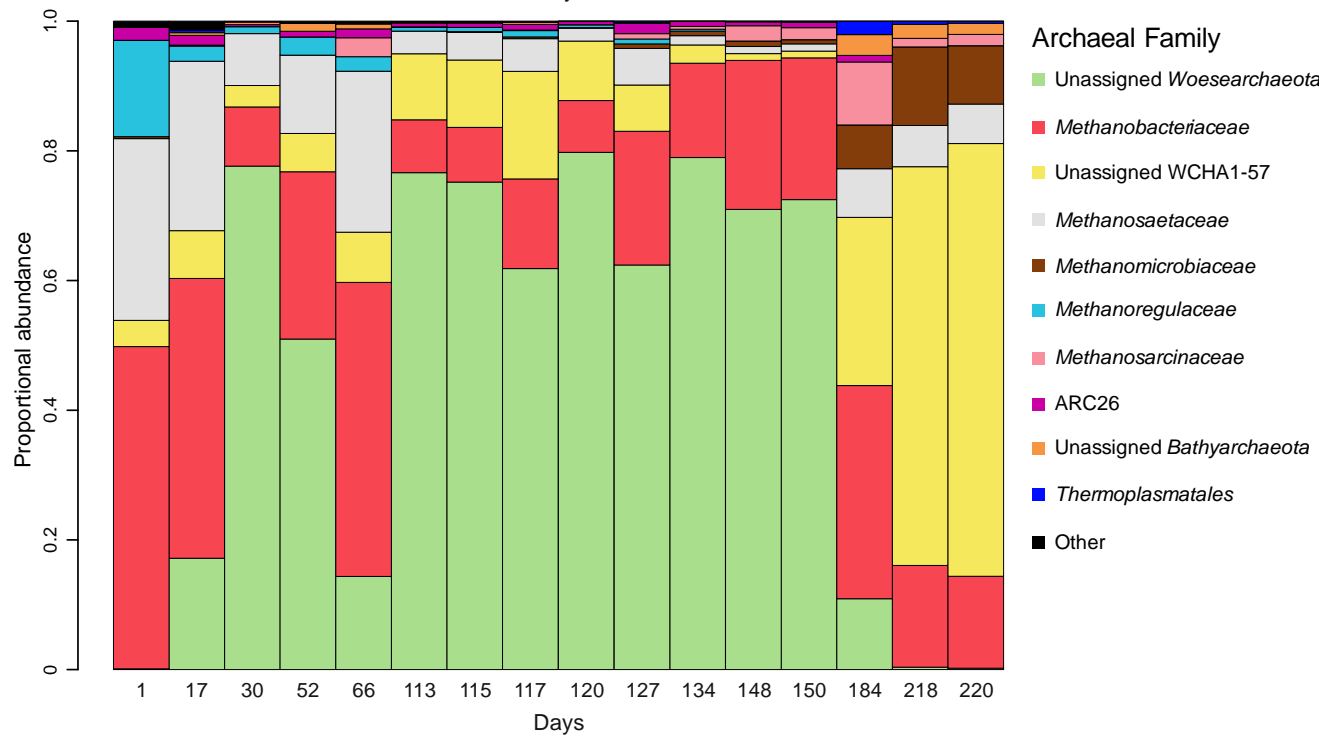

Figure 6.3. Bacterial (a) and archaeal (b) community dynamics in the AnMBR. Phylogenetic annotation at family level with abundance $>1 \%$ in at least one sample. Unassigned was used to indicate groups that could not be classified at family level. "Other" integrates the remaining families with less than $1 \%$ of relative abundance.

In this context, but bringing back the discussion to Porphyromonadaceae family, a proteolytic strain isolated from granular sludge of a UASB reactor treating brewery wastewater, Proteiniphilum acetatigenes, accelerated the propionate-degradation rate of a methanogenic propionate-degrading syntrophic coculture (Chen and Dong, 2005). Therefore, it is interesting to speculate about the role of Porphyromonadaceae and unassigned Cloacimonetes in our AnMBR, since an interaction between 
proteolysis, amino acid degradation and syntrophic methanogenesis is highly probable in the $\mathrm{AD}$ of pot ale.

Syntrophic acetate oxidation (SAO) is the predominant pathway for methane production in anaerobic digestion processes high in ammonia and volatile fatty acids (Schnürer et al., 1999). Therefore, the lack of Clostridia was unexpected since many SAO members belong to this class. For instance, Thermacetogenium phaeum, Tepidanaerobacter acetatoxydans, Clostridium ultunense and Syntrophaceticus schinkii. Nevertheless, recent studies have shown that syntrophic acetate oxidizers are phylogenetically diverse and not restricted to Clostridia only (Müller et al., 2016; Westerholm et al., 2016). For instance, Spirochaetes have been associated with syntrophic acetate oxidation (Lee et al., 2015).

\section{Archaeal community dynamics}

The inoculum sludge showed a dominance of hydrogenotrophic methanogens with a relative abundance of 50\% of Methanobacteriaceae and 15\% of Methanoregulaceae. The acetoclastic methanogens were represented by Methanosaetaceae (28\%). Methanobacteriaceae persisted as the most important methanogenic family, occasionally the relative abundance dropped to less than $10 \%$ during the start-up stage, but levels between 21 and 33\% were common during the stable operation of the AnMBR. Methanoregulaceae on the other hand quickly disappeared during the start-up stage and it was not detected during the stable operation of the reactor.

The relative abundance of Methanosaetaceae significantly decreased after the startup stage. Acetoclastic methanogens are more susceptible to ammonia inhibition than hydrogenotrophic methanogens, therefore the high levels of ammonia during the start-up stage might have contributed to reduce the abundance of Methanosaetaceae. During stable operation (days 134-150) the relative abundance of Methanosaetaceae did not surpass the $1 \%$, while only $6-8 \%$ was reached after day 184 .

The unassigned Woesearchaeota became relevant soon after the beginning of operation of the reactor on day 17 , and dominated the archaeal community since day 30 with relative abundance levels generally $>70 \%$. Woesearchaeota has been mostly reported in saline habitats and sediments, but it has also been detected in sludge (Ortiz-Alvarez and Casamayor, 2016). Recent single-cell genome re-construction analyses in members of Woesearchaeota showed small genomes sizes and the lack of core biosynthetic pathways, suggesting that these archaea may have a symbiotic or parasitic lifestyle (Castelle et al., 2015). Although the information available from Woesearchaeota is limited, genomic analysis of members of this group (Castelle et al., 2015) allow us to suggest that the role of unassigned Woesearchaeota in our bioreactor is of a fermentative and hydrogen-producing archaeon. 
The relative abundance of unassigned WCHA1-57 increases during the start-up stage from $4 \%$ in the inoculum to $17 \%$ on day 117 . After a significant decrease of the relative abundance to only $1 \%$ on day 150 , the relative abundance of unassigned WCHA1-57 increases to dominates the archaeal community at the last stage of operation of the reactor. The uncultured archaeal group WCHA1-57 (also called WSA2 or ARC I) may represent a new order of hydrogenotrophic methanogens that contributes to methane production in anaerobic digesters (Saito et al., 2015).

Similar to our results, WCHA1-57 and Methanobacterium were the dominant methanogens in a pilot-scale AnMBR operated at extremely short HRT (Mei et al., 2017). Moreover, WCHA1-57 has been reported as the predominant archaeal component $(>70 \%)$ in anaerobic digesters treating municipal sewage sludge (Chouari et al., 2005).

Interestingly, the relative abundances of unassigned WCHA1-57 and unassigned Woesearchaeota, are contrasting along the operational stages of the AnMBR. When the relative abundance of Woesearchaeota are $>70 \%$, the relative abundance of unassigned WCHA1-57 does not exceed the 10\%; and only when the relative abundance of unassigned Woesearchaeota drops to $<11 \%$, unassigned WCHA1-57 take over as the dominant OTU in the archaeal community ( $>60 \%)$. We can only speculate if these groups of archaea endure competition for some common substrates. Nevertheless, we have suggested some lines above a hydrogen-producing role of unassigned Woesearchaeota and a hydrogenotrophic role of unassigned WCHA1-57, therefore other factors influencing the antagonism between these groups should be considered.

\section{The effect of HLT to microbial community change}

There is an important shift of the archaeal and bacterial community between days 66 and 113, as well as between days 150 and 184. The performance of the reactor indicates that on day 78 the total-VFA values accumulated up to $19 \mathrm{meq} \cdot \mathrm{l}^{-1}$ (Figure 6.1). During the start-up period several operational problems occurred, in this case on day 77 the reactor was fed with vinasse as the pot ale from the distillery was not available. The increase in VFA and the shift in the microbial community seem to be related to this event.

Between days 150 and 184, another important shift in the bacterial and archaeal communities took place. This coincides with the period in which the high load experiments were performed (days 149 and 156). After the third HLT, the relative abundance of Methanomicrobiaceae increased to important levels (12\%). Also, the relative abundance of Methanosaeta increased from 1\% before the HLTs to $6-8 \%$ during the last stage of operation. Among bacteria, unassigned Bacteroidia and 
unassigned Cloacimonetes co-dominated the bacterial community in the reactor after the HLTs.

\section{Conclusions}

The reactor showed excellent performance with regard to COD removal and effluent quality. Moreover, it proved to be a robust system able to cope with the short high loading levels in the VLR during the HLTs and in a longer term in the last month of operation. The microbial population was able to withstand changes in hydrogen concentrations and total-VFA accumulation, with some groups of microorganisms taking over other groups while maintaining an overall good performance of the reactor.

Hydrogenotrophic methanogens dominated the methanogenic community in the reactor. Considering that pot ale is a protein rich feed, it seems plausible that acetoclastic microorganisms were inhibited by the high levels of ammonia. Not only the relative abundance of acetoclastic methanogens was low, but also the lack of Clostridia, which includes many SAO members, was unexpected. Therefore, other acetoclastic microorganisms, yet unknown, may have had a role in acetate degradation which is important in high ammonia anaerobic digestion processes.

The detection of members of all metabolic groups in the anaerobic degradation chain (hydrolytic, fermentative, syntrophic, acetogenic and methanogenic) illustrates the importance of a balanced and diverse biomass to have a robust and stable methanogenic reactor. Furthermore, the high relative abundance of uncultured groups of bacteria and archaea shows the potential for exploring the functions of novel uncultured microorganisms.

The monitoring of hydrogen concentrations in the biogas might be insufficient as a stand-alone control variable for anaerobic digestion, but its rapid response and ease of on-line measurement supports its use in digester control along with other liquid phase parameters to be measured on-line, for instance VFA or dissolved $\mathrm{H}_{2}$.

\section{Acknowledgments}

This research was supported by the Dutch Technology Foundation (STW) (project 11603), which is part of the Netherlands Organization for Scientific Research (NWO), and which is partly funded by the Ministry of Economic Affairs. Research of AJMS is supported by the European Research Council (ERC grant 323009) and the Gravitation grant (024.002.002) of the Netherlands Ministry of Education, Culture and Science. 


\section{Supporting Information}

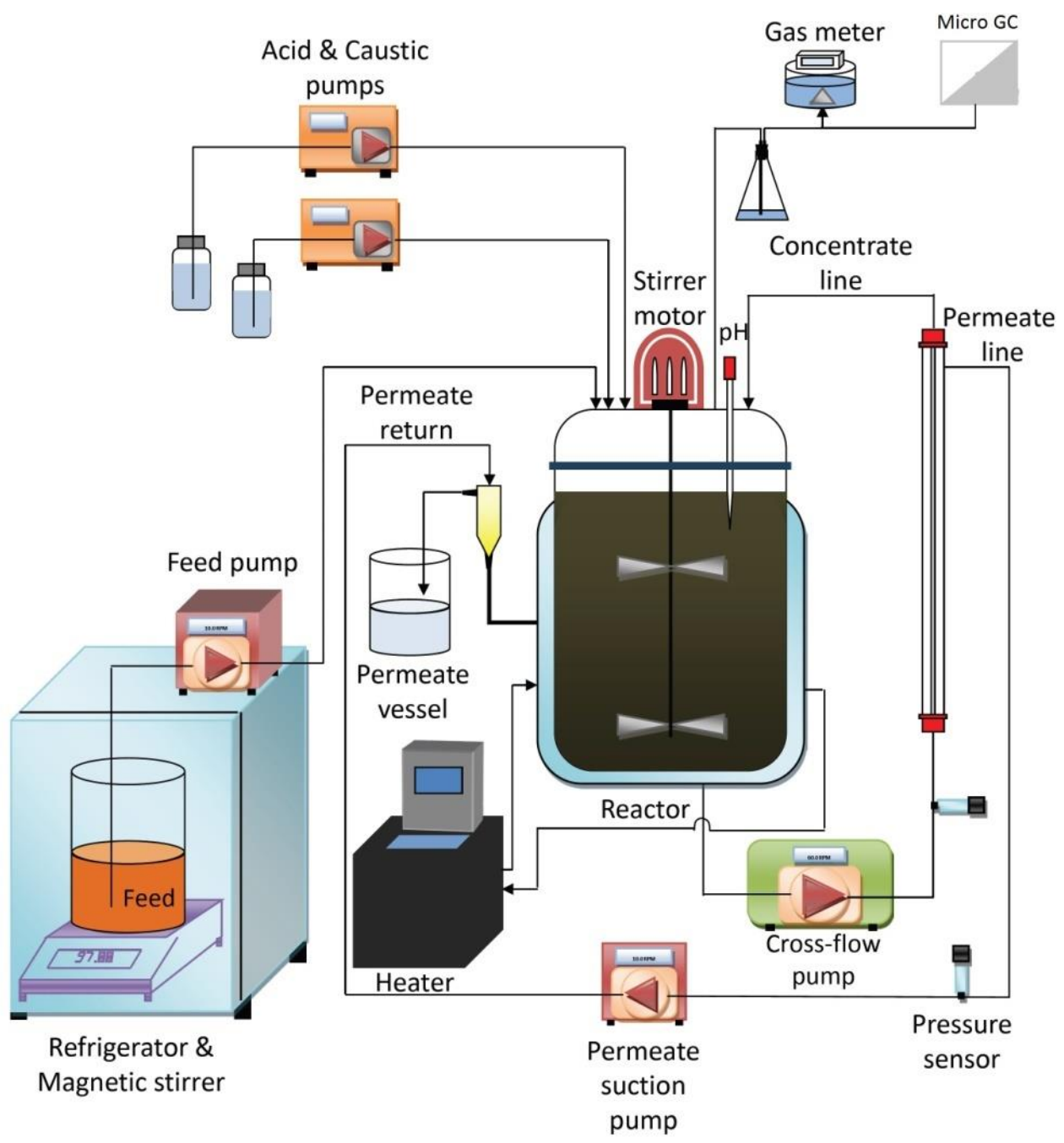

Fig. S6.1. Lab-scale reactor set-up schematic representation. Modified from (Dereli et al., 2015) 

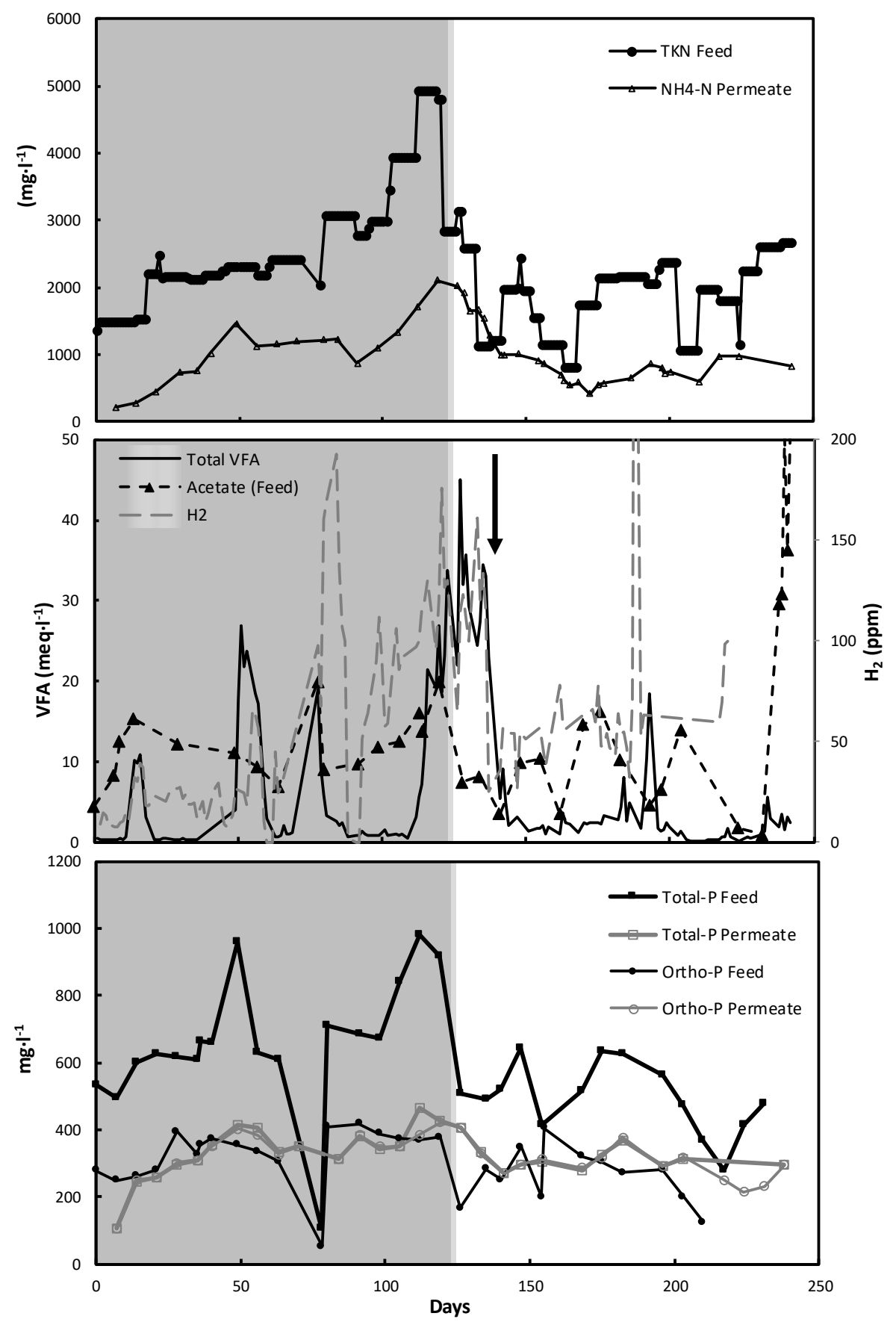

Fig. S6.2. TKN, VFA, $\mathrm{H}_{2}$ and $\mathrm{P}$ concentrations in the pot ale (feed), permeate and biogas. The grey area indicates the period when undiluted pot ale was used. The black arrow marks the point when the reactor was fed with $\mathrm{NaHCO}_{3}$ to wash away the high VFA levels. 


\begin{tabular}{|l|c|c|c|}
\hline \multicolumn{5}{|l|}{ Table S6.1. General composition of the pot ale. } \\
\hline \multicolumn{1}{|c|}{ Parameters } & Values $\left(\mathbf{m g} \cdot \mathbf{l}^{-\mathbf{1}}\right.$ ) & Parameters & Values $\mathbf{( m g} \cdot \mathbf{l}^{\mathbf{1}} \mathbf{)}$ \\
\hline Total COD & $62,241 \pm 7,572$ & Ammonium $\left(\mathrm{NH}_{4}\right)$ & $80 \pm 9$ \\
\hline Soluble COD & $52,336 \pm 4,926$ & Sulfate & $163 \pm 53$ \\
\hline Total Solids (TS) & $54,540 \pm 12,090$ & Chlorides & $383 \pm 15$ \\
\hline Volatile Solids (VS) & $50,659 \pm 11,718$ & Calcium & $76 \pm 77$ \\
\hline Total Suspended Solids (TSS) & $24,638 \pm 11,022$ & Magnesium & $165 \pm 62$ \\
\hline Volatile Suspended Solids (VSS) & $23,238 \pm 10,327$ & Potassium & $1036 \pm 57$ \\
\hline Total phosphorous $\left(\mathrm{P}_{\text {total }}\right)$ & $603 \pm 74$ & Sodium & $33 \pm 14$ \\
\hline Phosphate $\left(\mathrm{PO}_{4}\right)$ ) & $352 \pm 44$ & Copper & $1.5 \pm 0.8$ \\
\hline Total Kjeldahl Nitrogen (TKN) & $2,170 \pm 581$ & $\mathrm{pH}$ & $4 \pm 0.4$ \\
\hline Soluble Kjeldahl Nitrogen $(\mathrm{SKN})$ & $1,158 \pm 93$ & & \\
\hline
\end{tabular}

\begin{tabular}{|c|c|c|c|c|c|c|c|}
\hline \multirow{2}{*}{$\begin{array}{c}\text { Reactor } \\
\text { Stage }\end{array}$} & \multirow{2}{*}{ Sample ${ }^{1}$} & \multicolumn{3}{|c|}{ Bacterial } & \multicolumn{3}{|c|}{ Archaeal } \\
\hline & & Total & Filtered $^{2}$ & Merged $^{3}$ & Total & Filtered $^{2}$ & Merged $^{3}$ \\
\hline \multirow{2}{*}{0} & $\mathrm{~A}_{1}$ & 242444 & 236424 & \multirow{2}{*}{666511} & 4803 & 4767 & \multirow{2}{*}{18575} \\
\hline & $\mathrm{A}_{2}$ & 442591 & 430087 & & 13858 & 13808 & \\
\hline \multirow{16}{*}{ I } & $\mathrm{B}_{1}$ & 249235 & 238637 & \multirow{2}{*}{800548} & 14076 & 9801 & \multirow{2}{*}{44836} \\
\hline & $\mathrm{B}_{2}$ & 616068 & 561911 & & 41312 & 35035 & \\
\hline & $\mathrm{C}_{1}$ & 337600 & 314388 & \multirow[b]{2}{*}{607939} & 171263 & 114533 & \multirow[b]{2}{*}{190710} \\
\hline & $\mathrm{C}_{2}$ & 321540 & 293551 & & 112787 & 76177 & \\
\hline & $\mathrm{D}_{1}$ & 498373 & 493030 & \multirow{2}{*}{652482} & 9600 & 9300 & \multirow{2}{*}{33321} \\
\hline & $\mathrm{D}_{2}$ & 161738 & 159452 & & 25828 & 24021 & \\
\hline & $\mathrm{E}_{1}$ & 204733 & 198876 & \multirow{2}{*}{481556} & 17211 & 16543 & \multirow{2}{*}{27420} \\
\hline & $\mathrm{E}_{2}$ & 289208 & 282680 & & 11576 & 10877 & \\
\hline & $\mathrm{F}_{1}$ & 200737 & 192240 & \multirow{2}{*}{426786} & 25103 & 21514 & \multirow{2}{*}{104299} \\
\hline & $\mathrm{F}_{2}$ & 240690 & 234546 & & 94523 & 82785 & \\
\hline & $\mathrm{G}_{1}$ & 425210 & 412879 & \multirow{2}{*}{547029} & 35266 & 30002 & \multirow{2}{*}{76621} \\
\hline & $\mathrm{G}_{2}$ & 137928 & 134150 & & 50872 & 46619 & \\
\hline & $\mathrm{H}_{1}$ & 221170 & 210949 & \multirow{2}{*}{593192} & 30994 & 28982 & \multirow{2}{*}{99382} \\
\hline & $\mathrm{H}_{2}$ & 397467 & 382243 & & 73216 & 70400 & \\
\hline & $\mathrm{I}_{1}$ & 355596 & 340924 & \multirow{2}{*}{613384} & 50728 & 48494 & \multirow{2}{*}{92826} \\
\hline & $\mathrm{I}_{2}$ & 284037 & 272460 & & 46220 & 44332 & \\
\hline \multirow{4}{*}{ II } & $\mathrm{J}_{1}$ & 189576 & 180899 & \multirow[b]{2}{*}{280942} & 30211 & 28697 & \multirow{2}{*}{70925} \\
\hline & $\mathrm{J}_{2}$ & 104329 & 100043 & & 44209 & 42228 & \\
\hline & $\mathrm{K}_{1}$ & 154978 & 149089 & 45108 & 12627 & 11391 & 66008 \\
\hline & $\mathrm{K}_{2}$ & 314081 & 302535 & 401024 & 60200 & 54707 & 00090 \\
\hline JI & $\mathrm{L}_{1}$ & 91558 & 90680 & 50725 & 70502 & 66119 & 141 \\
\hline 111 & $\mathrm{~L}_{2}$ & 425552 & 416674 & 501004 & 80774 & 75365 & 141484 \\
\hline IV & $\mathrm{M}_{1}$ & 427718 & 417505 & 703964 & 88254 & 84630 & 111166 \\
\hline IV & $\mathrm{M}_{2}$ & 290848 & 286459 & 103964 & 28163 & 26536 & 111100 \\
\hline $\mathrm{V}$ & $\mathrm{N}_{1}$ & 347177 & 333540 & 795027 & 11951 & 9948 & 26796 \\
\hline$v$ & $\mathrm{~N}_{2}$ & 488748 & 461487 & 190028 & 18107 & 16848 & 20190 \\
\hline VI & $\mathrm{O}_{1}$ & 61409 & 60914 & 263916 & 20949 & 17919 & 40967 \\
\hline$\sqrt{1}$ & $\mathrm{O}_{2}$ & 205644 & 203002 & 200510 & 31738 & 23048 & 40501 \\
\hline VII & $\mathrm{P}_{1}$ & 416891 & 413673 & 869154 & 60005 & 46886 & 143985 \\
\hline VII & $\mathrm{P}_{2}$ & 458567 & 455481 & 809104 & 117672 & 97099 & 140500 \\
\hline Av & age & 300107 & 289419 & 578838 & 47019 & 40294 & 80588 \\
\hline Nom & & chn & $\begin{array}{l}\text { licate } \\
\text { igned }\end{array}$ & -5 & 1. & & \\
\hline
\end{tabular}


Table S6.3. Taxonomic assignations of the reads obtained by NGS analysis.

\begin{tabular}{|c|c|c|c|c|}
\hline \multicolumn{5}{|c|}{ BACTERIA } \\
\hline PHYLUM & CLASS & ORDER & FAMILY & GENUS \\
\hline Actinobacteria & Actinobacteria & Acholeplasmatales & Acholeplasmataceae & Acinetobacter \\
\hline Aminicenantes & Anaerolineae & Anaerolineales & Alcaligenaceae & Alcaligenes \\
\hline Bacteroidetes & Bacilli & Bacillales & Anaerolineaceae & Geobacter \\
\hline Caldiserica & Bacteroidetes & Bacteroidales & Anaerolineaceae & Lactobacillus \\
\hline Chlorobi & Bacteroidia & Bacteroidia & Bacteroidaceae & Leuconostoc \\
\hline Chloroflexi & Betaproteobacteria & Burkholderiales & Campylobacteraceae & Longilinea \\
\hline Cloacimonetes & BSA1B-03 & Caldisericales & CAP-aah99b04 & Macellibacteroides \\
\hline Elusimicrobia & Caldisericia & Campylobacterales & Christensenellaceae & Mesotoga \\
\hline Firmicutes & Candidatus & Chlorobiales & Clostridiales & Prevotella \\
\hline Ignavibacteriae & Chlorobia & Clostridiales & Comamonadaceae & Proteiniphilum \\
\hline Microgenomates & Cloacimonetes & Desulfuromonadales & Erysipelotrichaceae & Pseudomonas \\
\hline Nitrospirae & Clostridia & Erysipelotrichales & Eubacteriaceae & Romboutsia \\
\hline Proteobacteria & Deltaproteobacteria & Ignavibacteriales & Geobacteraceae & Syntrophobacter \\
\hline Spirochaetae & Elusimicrobia & Kosmotogales & Helicobacteraceae & Uncultured \\
\hline Synergistetes & Epsilonproteobacteria & Lactobacillales & Ignavibacteriaceae & \\
\hline Tenericutes & Erysipelotrichia & Methylophilales & Kosmotogaceae & \\
\hline Thermotogae & Gammaproteobacteria & NB1-n & Lachnospiraceae & \\
\hline \multirow[t]{28}{*}{ Verrucomicrobia } & Ignavibacteria & Nitrospirales & Lactobacillaceae & \\
\hline & LD1-PB3 & Petrotogales & Lentimicrobiaceae & \\
\hline & LNR & Propionibacteriales & Leuconostocaceae & \\
\hline & Mollicutes & Pseudomonadales & M2PB4-65 & \\
\hline & Nitrospira & Sphingobacteriales & Marinilabiaceae & \\
\hline & OPB35 & Spirochaetales & Methylophilaceae & \\
\hline & Sphingobacteriia & Synergistales & Moraxellaceae & \\
\hline & Spirochaetes & Syntrophobacterales & Nitrospiraceae & \\
\hline & Synergistia & Thermoanaerobacterale & OPB56 & \\
\hline & Thermotogae & Uncultured & Peptostreptococcaceae & \\
\hline & W27 & & Petrotogaceae & \\
\hline & W5 & & PHOS-HE36 & \\
\hline & Uncultured & & Planococcaceae & \\
\hline & & & Porphyromonadaceae & \\
\hline & & & Prevotellaceae & \\
\hline & & & Prolixibacteraceae & \\
\hline & & & Propionibacteriaceae & \\
\hline & & & Pseudomonadaceae & \\
\hline & & & Rikenellaceae & \\
\hline & & & Ruminococcaceae & \\
\hline & & & Spirochaetaceae & \\
\hline & & & ST-12K33 & \\
\hline & & & Synergistaceae & \\
\hline & & & Syntrophaceae & \\
\hline & & & Syntrophobacteraceae & \\
\hline & & & WCHB1-02 & \\
\hline & & & Uncultured & \\
\hline & \multicolumn{4}{|c|}{ ARCHAEA } \\
\hline PHYLUM & CLASS & ORDER & FAMILY & GENUS \\
\hline Bathyarchaeota & Methanobacteria & Methanobacteriales & ARC26 & Methanobacterium \\
\hline Euryarchaeota & Methanomicrobia & Methanomicrobiales & GOM & Methanoculleus \\
\hline Woesearchaeota & Thermoplasmata & Methanosarcinales & Methanobacteriaceae & Methanolinea \\
\hline \multirow[t]{7}{*}{ WSA2 } & WCHA1-57 & Thermoplasmatales & Methanomicrobiaceae & Methanomethylovorans \\
\hline & Uncultured & Uncultured & Methanoregulaceae & Methanosaeta \\
\hline & & & Methanosaetaceae & Methanosarcina \\
\hline & & & Methanosarcinaceae & Methanospirillum \\
\hline & & & Methanospirillaceae & Uncultured \\
\hline & & & Thermoplasmatales & \\
\hline & & & & \\
\hline
\end{tabular}


"There is only certainty with respect to the past, and with respect to the future, the certainty of death" Erich Fromm 

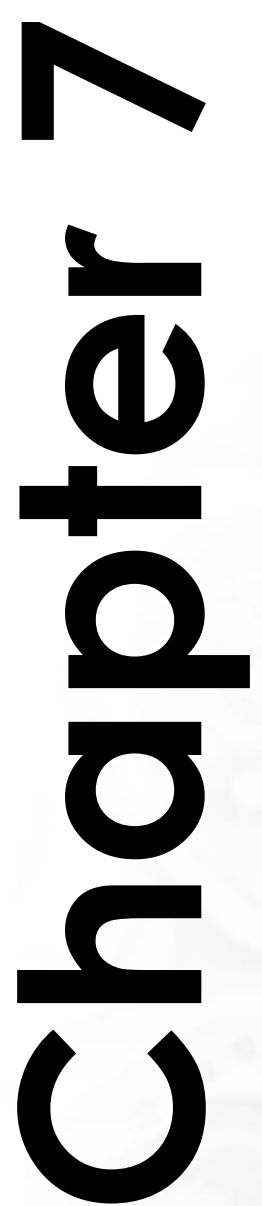


\section{CHAPTER 7}

\section{GENERAL DISCUSSION}


Phenotypically distinct microorganisms may form obligate syntrophic interactions because they are metabolically dependent on each other in certain conditions (Schink, 1997; McInerney et al., 2008; Stams and Plugge, 2009; Morris et al., 2013). The low energy yield associated with fatty acid degradative pathways can only be conserved by some microorganisms via specialized biochemical mechanisms. Despite the ecological importance of the syntrophic interactions, our understanding of the molecular basis of syntrophic lifestyle remains limited. A better understanding of how microorganisms cope with energetic constraints is important to provide new insights into methane production, waste treatment, and to engineer biotechnological processes or to design synthetic enzymes for the catalysis of energetically unfavourable reactions.

The research presented in this thesis focussed on the molecular mechanisms used by Syntrophobacter fumaroxidans, a propionate-oxidizing bacterium, and its methanogenic partners Methanospirillum hungatei and Methanobacterium formicicum. An emphasis was put on the interspecies electron transfer (IET) and the importance of formate as an interspecies electron carrier in syntrophic propionate degradation. In addition, alternative energy conservation mechanisms and their role in sulfate reduction and methanogenesis are discussed. Genome and proteome analyses revealed a detailed description of propionate degradation by Syntrophobacter fumaroxidans (Chapter 4) as well as of the methanogenic metabolism of two hydrogenotrophic methanogens, Methanospirillum hungatei and Methanobacterium formicicum, grown in syntrophic association and in pure cultures (Chapter 5).

In Chapter 2 the occurrence of genetic markers for syntrophic growth on butyrate and propionate were explored in the genomes of short chain fatty acid degraders known to engage in syntrophy with methanogens. The domain-based functional profiling analyses shows that the presence of periplasmic formate dehydrogenases and their maturation proteins FdhE in the genome of syntrophs is a difference with the non-syntrophic butyrate and propionate degraders.

A previous genome comparison study of syntrophic bacteria reports that confurcating hydrogenases and membrane-associated reverse electron transport (RET) complexes are present in syntrophs and that they play a critical role in syntrophy (Sieber et al., 2012). The analyses presented in Chapter 2 confirms the importance of membrane associated RET complexes, like the Rnf or Ech complexes. However, the presence of confurcating hydrogenases in non-syntrophic bacteria, like Desulfotomaculum kuznetsovii and Desulfobulbus propionicus, indicates that these complexes are not exclusive for syntrophs and can also be important in energy conservation in nonsyntrophic bacteria. The analysis of Sieber and co-workers was restricted to genomes of syntrophs whereas the analysis in Chapter 2 of this thesis also included genomes of non-syntrophic sulfate-reducing bacteria (SRB). However, not many of those SRB 
have been tested for syntrophic butyrate or propionate degradation. Only Desulfotomaculum kuznetsovii and Desulfobulbus propionicus are validated nonsyntrophic bacteria. To strengthen the importance of the analysis, more butyrateand propionate-degrading SRB should be tested for syntrophic capability.

Moreover, the combination of more than one molecular mechanism as a characteristic of syntrophs was not completely explored in Chapter 2. For instance, it has been proposed that the proton-translocating pyrophosphatase (HppA) in SRB has a role in energy conservation by proton translocation and hydrolysis of pyrophosphate (Cypionka, 1995; Baltscheffsky et al., 1999). A reverse use of this transmembrane protein to conserve energy could be feasible (Serrano et al., 2007). The HppA, in contrast with Rnf, was ubiquitously present in all the analysed syntrophic microorganisms in Chapter 2, even in the non-sulfate reducers such as Syntrophomonas wolfei. It can be argued, as in the case of the confurcating hydrogenases, that although HppA is not present in D. propionicus, it is present in the genome of the non-syntroph $D$. kuznetsovii, therefore HppA is not a genetic marker for syntrophic growth. However, if we hypothesise that syntrophs need both: complexes for RET, such as HppA or Rnf, and complexes that facilitate IET, we observed that neither of the validated non-syntrophic bacteria fulfils both conditions. Although the genome of $D$. kuznetsovii revealed the presence of HppA, it lacks periplasmic formate dehydrogenases and periplasmic hydrogenases (Visser et al., 2013). Whereas, D. propionicus despite having genes coding for periplasmic hydrogenases, lacks proton translocating mechanisms like Rnf or HppA. Therefore, butyrate- and propionate-degrading bacteria must need both RET and IET mechanisms to be able to grow in syntrophy.

Although the genes suspected to be exclusive in syntrophic microorganisms correspond to those coding for energy metabolism, other protein domains putatively involved in the formation of spatial structures such as capsule or biofilm (IPR019079) and cell-shape determination (IPR018365) were also highlighted to be important for syntrophic growth. Numerous studies have found genes involved in the formation of spatial structures such as biofilm, granule formation and flagella and pili synthesis, to be important for syntrophic interactions (Kato and Watanabe, 2010; Summers et al., 2010; Krumholz et al., 2015). However, many biofilm formation and flagellar proteins are also produced during axenic growth (Nadell et al., 2009; Clark et al., 2012). Although cellular aggregation and the structure of a mixed community might facilitate the exchange of metabolites between cells (Ishii et al., 2005; Shimoyama et al., 2009; Brileya et al., 2014), these are not essential attributes in the formation and maintenance of syntrophy, as the interspecies electron transfer.

It is presented in Chapter 2 that phylogeny does not predict syntrophy, nonetheless other potential genomic markers for syntrophy have been investigated. Recently, a genetic polymorphism has been described where only a specific genotype of 
Desulfovibrio vulgaris is able to engage in syntrophy with Methanococcus maripaludis (Grosskopf et al., 2016). Interestingly, the reported genetic alterations in the syntrophic genotype are not related to interspecies electron transfer but involved in RET during lactate oxidation. One mutation affects the enzymes that catalyse lactate uptake and the conversion of pyruvate, while the second mutation is affecting the $\mathrm{H}^{+} / \mathrm{Na}^{+}$ion-translocating subunit of a membrane-bound dehydrogenase. The authors proposed that the identified polymorphism in the latter gene increases the number of ions which can translocate over the membrane per number of hydrogen produced. Thereby, the cell can use the membrane gradient as a form of cellular energy to invest in lactate oxidation (Grosskopf et al., 2016). This hypothesis reinforces the importance of proton translocating mechanisms in syntrophic bacteria. Furthermore, it glances at the differential capacities of proton translocating mechanisms present in syntrophic bacteria, to transport protons more efficiently than in non-syntrophic bacteria. A similar energy conservation concept has been proposed before by hypothesising that the smallest quantum of energy that can be transported via electron transfer phosphorylation by the ATP synthase is lower in syntrophs than in non-syntrophic bacteria (Worm, 2010).

The maximum number of protons that have to be translocated across the membrane for ATP synthesis, or hydrolysis in RET, is determined by the number of c-subunits in the membrane integrated $\mathrm{F}_{0}$ region of the ATP synthase (Nakanishi-Matsui and Futai, 2006). With one full rotation of the ATP synthase complex, each of the three catalytic $B$ subunits in the $\mathrm{F}_{1}$ region synthesizes/hydrolyses one ATP molecule, and each of the c-subunits in $\mathrm{F}_{0}$ transports one proton. (Nakanishi-Matsui et al., 2010; Soga et al., 2017). Among the bacteria analysed in Chapter 2, syntrophs contain smaller ATP synthase c-subunits than sulfate reducers. Thus, in principle, the membrane integrated rotor in syntrophs may contain more c-subunits. A higher number of protons translocated per ATP hydrolysed would result in an increase of the smallest quantum of biologically conservable energy. Therefore, a c-subunit to ATP synthase ratio would give insight in the minimum amount of energy that can be conserved by syntrophs. Yet, this ratio is not known for the analysed bacteria in chapter 2. The size of the c-subunits discussed above are only predictions based on the amino acid sequences available in their genome. Biochemical and further proteomic analysis of ATP synthases are necessary to reinforce this hypothesis. Unfortunately, in the proteomic analysis in chapter 4 the c-subunit was not detected in any of the growth conditions.

Chapter 3 focused on the metabolic flexibility of $S$. fumaroxidans to grow in syntrophy with $M$. hungatei or $D$. desulfuricans in a sulfate-rich medium. In general, sulfate reduction is favoured over methanogenesis when sufficient sulfate is present (Lovley and Klug, 1983; Muyzer and Stams, 2008). Our results showed sulfate 
reduction by $S$. fumaroxidans, but propionate oxidation coupled to hydrogen and formate production also occurred at enough levels to sustain $D$. desulfuricans growth.

In this thesis, the capacity of sulfate reducers to grow in syntrophy has been discussed (Chapter 2 \& 3). Several comparative transcriptomic analyses have been performed to find out the key genetic elements for syntrophy by considering the metabolic flexibility of SRB (Walker et al., 2009; Plugge et al., 2010; Plugge et al., 2011). However, in those studies sulfate was added to syntrophic cocultures to assess the change in gene expression. Chapter 3 also included a perturbation in sulfatereducing cultures towards syntrophic conditions by adding a hydrogen/formate scavenging microorganism. Syntrophic cocultures have been obtained before by adding an hydrogen scavenger (Boone and Bryant, 1980), however in our experimental approach the addition of the syntrophic partner did not involve a limitation of sulfate and the levels of sulfide were high. The study in Chapter 3 might have given different results if sulfate would have been limited. In the study of (Grosskopf et al., 2016), cultures of clones of D. vulgaris prone to engage in syntrophy produced more hydrogen during lactate oxidation compared to those clones with a non-syntrophic genotype. Nevertheless, this hydrogen accumulation was observed only when sulfate was not provided or when it was limited at 50\% (30 mM lactate and $7.5 \mathrm{mM} \mathrm{SO}_{4}{ }^{2-}$.

The sulfate-reducing capacities of members of Syntrophobacteracea have been shown in sulfate-perturbed methanogenic environments (Liu and Conrad, 2017). Yet, the growth rate of $S$. fumaroxidans with propionate and sulfate is much slower than when grown in syntrophy with methanogens (van Kuijk and Stams, 1995; Scholten and Conrad, 2000). The slow growth rates as sulfate reducers, or even the lack of ability to respire sulfate, of some members of Syntrophobacterales, such as Smithella spp. and Syntrophus spp., has led to the speculation that these bacteria might be losing the ability for sulfate respiration after dealing with low concentrations of sulfate in methanogenic environments (Plugge et al., 2011). Moreover, evolutionary experiments support these theories. It has been shown that the mutations that cause the specialization for syntrophy, result in detriment of sulfate-reducing capacities in cocultures of $D$. vulgaris and $M$. maripaludis (Hillesland and Stahl, 2010; Hillesland et al., 2014). In Chapter 3 we assessed how prone $S$. fumaroxidans is to grow in syntrophy despite the availability of enough sulfate to grow on its own, thus indirectly estimating the hypothetical loss of sulfate-reducing capacities of our model bacterium. It is remarkable that even in a sulfate-rich medium $S$. fumaroxidans maintained a syntrophic relationship with a hydrogen/formate scavenger. In Chapter 4 it was observed that most of the enzymes required for sulfate reduction were present in all growth conditions. Therefore, the prevalence of propionate oxidation coupled to proton and $\mathrm{CO}_{2}$ reduction in sulfate-rich medium also points to 
the fact that the molecular mechanisms for energy conservation available in $S$. fumaroxidans genome, are ubiquitously produced.

Nevertheless, the ubiquitous production by $S$. fumaroxidans of proteins involved in sulfate reduction and in hydrogen and formate production, might be an advantageous trait. Recently, an evolutionary experiment in cocultures of D. vulgaris and $M$. maripaludis was done with fluctuating availability of sulfate (Turkarslan et al., 2017). Results showed that when sulfate availability fluctuated too frequently in an environment with excess lactate and the abundance of methanogens, the gene regulation in $D$. vulgaris to shift repeatedly between sulfate-reducing and syntrophic physiologies drove the cultures to collapse. It was concluded that transcription regulation can be detrimental in a rapidly fluctuating environment.

Biochemical, genomic and transcriptomic analysis of Syntrophobacter fumaroxidans has been performed (de Bok et al., 2002b; Müller et al., 2010; Worm et al., 2011b; Plugge et al., 2012). Chapter 4 widened our knowledge of the molecular mechanisms for energy conservation used by $S$. fumaroxidans during propionate degradation under different growth conditions. The importance of formate as interspecies electron carrier in $S$. fumaroxidans has been demonstrated before in cocultures with $M$. hungatei (de Bok et al., 2002a). Our results furthermore identified a set of three formate dehydrogenases (Fdh3, Fdh4 and Fdh5) that transfer electrons to the syntrophic partner. Two formate dehydrogenases (Fdh1 and Fdh2) have been purified from $S$. fumaroxidans (de Bok et al., 2003). Both enzymes were produced in fumarate-grown cells as well as in cells grown syntrophically on propionate with $M$. hungatei. Our proteomic results showed that Fdh1 and Fdh2 are the main formate dehydrogenases ubiquitously produced in propionate-degrading cultures, which is in agreement with the study of de Bok for the syntrophically grown cells with $M$. hungatei and suggest a similar production of these two enzymes during fumarate fermentation.

Purification of $S$. fumaroxidans Fdh1 and Fdh2 showed that both enzymes are tungsten (W) containing (de Bok et al., 2003). A following study revealed that growth in the presence of $\mathrm{W}$ led to an increase in total FDH activity relative to growth with molybdenum (Mo), either in coculture with $M$. hungatei or in axenic growth with propionate and fumarate (Plugge et al., 2009). The presence of both trace elements (W and Mo) on the other hand decreased total FDH activity in propionate and fumarate-grown cultures, which suggested an antagonistic effect of Mo in Wcontaining FDH. Remarkably, such effect was not observed for cells grown in coculture, which indicated the involvement of other FDHs than those known at the time of the study which were only the W-containing Fdh1 and Fdh2. Our proteomic results revealed that Fdh3, Fdh4 and Fdh5 are the formate dehydrogenase involved in IET. Moreover, these FDHs most probably contain Mo and can incorporate W, as the increase in total FDH activity with cocultured cells grown in the presence of W 
suggests. However, this assumption must be verified by purifying and characterizing the enzymes.

Enzyme activity studies dependent on the presence, absence or combination of $\mathrm{W}$ and Mo in the medium have been performed before in the model microorganisms studied in this thesis. In S. fumaroxidans the antagonistic effect of Mo in the W-FDHs has been discussed (Plugge et al., 2009). While for M. hungatei an antagonistic effect of Mo in the total FDH activity was observed in cells of this methanogen, which suggests that at least one of its $\mathrm{FDHs}$ (most probably W-containing) cannot incorporate Mo. The enzyme assays in $M$. hungatei cells grown with $\mathrm{H}_{2} / \mathrm{CO}_{2}$ or in coculture with $S$. fumaroxidans showed a higher total FDH activity when W and Mo were available in the growth medium than when only W was provided (Plugge et al., 2009). However, for formate grown cells the total FDH activity decreased with Mo presence in contrast to only-W supplemented medium. The proteomic analysis in chapter 5 showed that Fdh3 in $M$. hungatei is the only FDH that was more abundant in cells grown with formate than in cells grown with $\mathrm{H}_{2} / \mathrm{CO}_{2}$ or in coculture with $S$. fumaroxidans. Thus, we speculated that Fdh3 of $M$. hungatei might not be able to incorporate Mo, in contrast to the other W-FDHs.

In $M$. formicicum no antagonistic effect of $\mathrm{W}$ was reported, but this methanogen was not able to synthetize an active FDH during growth with W and lack of Mo (May et al., 1988). Chapter 5 revealed that the only FDH detected at the studied conditions of $M$. formicicum was Fdh1. Therefore, it is possible that this FDH is a Mo-containing enzyme. A better understanding of the implications of the metal content of FDHs, and other pterin enzymes such as formylmethanofuran dehydrogenases, in the interactions within methanogenic microbial communities might contribute to the optimization of metal dosage in anaerobic methanogenic bioreactors.

Regulatory mechanisms to express isoenzymes with different functions, under different conditions, with different cell locations, or incorporating different metals in the active site often allow the use of different pathways for energy conservation and adaptation to environmental constrains, such as substrate or metal availability (da Silva et al., 2013). For instance, enzymatic studies in D. vulgaris Hildenborough showed that a W-FDH is the main FDH in hydrogenotrophic conditions while the Mo-FDH was the most important FDH during growth with formate (da Silva et al., 2011). A following study with deletion mutants for the two main FDH detected in $D$. vulgaris provided the first direct evidence for the involvement of formate cycling during growth with lactate coupled to sulfate reduction (da Silva et al., 2013). In $S$. fumaroxidans the periplasmic Fdh2 might fulfil such role during growth with sulfate as showed in Chapter 4, besides the hydrogen cycling with Hyn. 
In Chapter 6, the performance and robustness to high loading tests of an anaerobic membrane bioreactor (AnMBR) were evaluated. The population analysis showed the presence of members of all metabolic groups of the anaerobic degradation chain which was essential for the robustness and stability of the reactor. The diversity of the microbial population permitted to some groups of microorganisms to take over other groups when changes in hydrogen concentrations and total-VFA accumulation were observed in the bioreactor. The abundance of uncharacterized microorganisms from known phyla and candidate phyla without cultivated representatives, shows the importance and urge for the isolation of novel uncultured microorganisms that permit us to investigate their functions in complex microbial communities.

Moreover, the results presented in Chapter 6 showed that the hydrogen concentrations can be used in digester control along with other liquid phase parameters to be measured on-line, for instance VFA or dissolved $\mathrm{H}_{2}$.

The importance of hydrogenases and formate dehydrogenases in the interactions of microorganisms present in methane producing environments has been discussed. But it is noteworthy to mention that there is an increasing interest in investigating the reversible biochemical processes of hydrogen and formate production, as well as the interconversion of these compounds for purposes of energy storage (Pereira, 2013). The developing of biocatalysts to produce reduced carbon compounds from $\mathrm{CO}_{2}$ has been proposed and investigated in the last decade (Reda et al., 2008; Mourato et al., 2017). $\mathrm{CO}_{2}$ removal from the atmosphere as a mean of relieving global warming while producing fuels or chemical feedstocks is an attractive possibility (El-Zahab et al., 2008; Yadav et al., 2012; Sakai et al., 2017) Moreover, the potential use of microorganisms as biocatalysts for $\mathrm{H}_{2}$ production from formate is currently in the spotlight for a future $\mathrm{H}_{2}$-based economy (Hambourger et al., 2008; Martins et al., 2015; Martins et al., 2016).

Thus, after decades of research, the enzymes that catalyse two of the simplest redox reactions in nature remain to be intensively studied and are strong candidates to facilitate new types of fuel cells and other technological developments in a post-oil society. 


\section{Future research}

- Biochemical and structural analysis of Fdh3, Fdh4 and Fdh5 of S. fumaroxidans is important as it could provide insight into the importance of molybdenum-dependent formate dehydrogenases during syntrophic growth.

- Sulfate-reducing bacteria such as Desulfobacterium autotrophicum, Desulfomonile tiedjei and Desulfosporosinus meridiei were never tested for syntrophic growth, but all crucial domains discussed in chapter 2 were found in the corresponding genomes, which suggests their possible ability to grow in syntrophic associations. Therefore, these bacteria should be tested for syntrophic growth. We attempted to establish a methanogenic syntrophic coculture by inoculating active $M$. hungatei in a lactate-grown culture of $D$. autotrophicum, but this was not successful. It is probable that the methanogen did not endure the high levels of sulfide in the medium. We suggest testing the above mentioned SRB for syntrophic growth with other sulfide resistant bacteria such as Desulfovibrio desulfuricans.

- The effect of short term overloading events in the microbial population of the AnMBR will be better evaluated by an RNA-based next generation sequencing which will allow to reveal the effect on the activity of specific microbial communities.

- Experimental evolution studies with slow-growing microorganisms such as $S$. fumaroxidans might be challenging, but the long term transferring of this bacterium growing under different conditions might be useful to evaluate inlab evolution (Hillesland and Stahl, 2010). A genetic comparison of de novo genome sequences of $S$. fumaroxidans cultures after several generations under different propionate-degrading conditions might lead to the detection of specific mutations that will help to understand the sulfate-reducing, syntrophic and fumarate respiratory genotype of this model bacterium.

- A phenomenon where only some subunits of multimeric enzymes seem to be produced in a modular way was frequently observed in the proteomic analyses discussed in this thesis. The molecular analysis of genes and proteins in this thesis showed that another level of molecular interactions shall be considered for discussion. Protein subunits and protein domains should be investigated as the building blocks that ultimately define the protein roles in the metabolism (Grein et al., 2013). 
- Hdr/Mvh-p is the most abundant putatively confurcating system during sulfate-reducing growth of $S$. fumaroxidans. This putative $\mathrm{Hdr}$ of $S$. fumaroxidans should be purified, its activity assessed and its role in sulfate reduction studied.

- Recently a classification system and web tool for the structural and functional analysis of hydrogenases has been developed (Søndergaard et al., 2016). The tool predictions for metal content, function and location of those hydrogenases present in S. fumaroxidans, M. hungatei and M. formicicum were correctly assigned in agreement with the roles suggested in this thesis. We suggest that the development of a similar web tool for an easy and faster analysis of formate dehydrogenases will be helpful to investigate the metabolism of syntrophic and sulfate-reducing bacteria.

- The metabolites exchanged during syntrophy are not exclusively restricted to electron transfer. The proteomic profiles of M. hungatei and M. formicicum showed that during syntrophic growth only a restricted set of proteins is produced compared to axenic growth on $\mathrm{H}_{2} / \mathrm{CO}_{2}$ or formate. Some biosynthetic pathways, such as biosynthesis of aromatic amino acids might not have been expressed during syntrophic growth in these methanogens. In this context, secondary syntrophy, where complementarity of amino acid metabolism takes place (Nobu et al., 2015), should be considered and further investigated in methanogenic communities. 


\section{REFERENCES}

Acharya, B.K., Mohana, S., and Madamwar, D. (2008). Anaerobic treatment of distillery spent wash - a study on upflow anaerobic fixed film bioreactor. Bioresource Technology 99, 4621-4626.

Albers, S.V., and Meyer, B.H. (2011). The archaeal cell envelope. Nature Reviews Microbiology 9, 414-426.

Altschul, S.F., Gish, W., Miller, W., Myers, E.W., and Lipman, D.J. (1990). Basic local alignment search tool. Journal of Molecular Biology 215, 403-410.

Anderson, I., Ulrich, L.E., Lupa, B., Susanti, D., Porat, I., Hooper, S.D., Lykidis, A., Sieprawska-Lupa, M., Dharmarajan, L., Goltsman, E., Lapidus, A., Saunders, E., Han, C., Land, M., Lucas, S., Mukhopadhyay, B., Whitman, W.B., Woese, C., Bristow, J., and Kyrpides, N. (2009). Genomic characterization of Methanomicrobiales reveals three classes of methanogens. PLoS One 4, e5797.

Angelidaki, I., and Ahring, B.K. (1993). Thermophilic anaerobic digestion of livestock waste: the effect of ammonia. Applied Microbiology and Biotechnology 38, 560-564.

Angelidaki, I., Ellegaard, L., and Ahring, B.K. (1993). A mathematical model for dynamic simulation of anaerobic digestion of complex substrates: focusing on ammonia inhibition. Biotechnology and Bioengineering 42, 159-166.

Aquino, S.F., Hu, A.Y., Akram, A., and Stuckey, D.C. (2006). Characterization of dissolved compounds in submerged anaerobic membrane bioreactors (SAMBRs). Journal of Chemical Technology and Biotechnology 81, 1894-1904.

Ariunbaatar, J., Scotto Di Perta, E., Panico, A., Frunzo, L., Esposito, G., Lens, P.N., and Pirozzi, F. (2015). Effect of ammoniacal nitrogen on one-stage and two-stage anaerobic digestion of food waste. Waste Management 38, 388-398.

Azman, S., Khadem, A.F., Van Lier, J.B., Zeeman, G., and Plugge, C.M. (2015). Presence and role of anaerobic hydrolytic microbes in conversion of lignocellulosic biomass for biogas production. Critical Reviews in Environmental Science and Technology 45, 2523-2564.

Baltazar, C.S.A., Marques, M.C., Soares, C.M., Delacey, A.M., Pereira, I.a.C., and Matias, P.M. (2011). Nickel-Iron-Selenium Hydrogenases - An Overview. European Journal of Inorganic Chemistry 2011, 948-962.

Baltscheffsky, M., Schultz, A., and Baltscheffsky, H. (1999). $\mathrm{H}^{+}$-PPases: a tightly membranebound family. FEBS Letters 457, 527-533.

Barrena, R., Traub, J.E., Gil, C.R., Goodwin, J.a.S., Harper, A.J., Willoughby, N.A., Sanchez, A., and Aspray, T.J. (2017). Batch anaerobic digestion of deproteinated malt whisky pot ale using different source inocula. Waste Management.

Bassegoda, A., Madden, C., Wakerley, D.W., Reisner, E., and Hirst, J. (2014). Reversible interconversion of $\mathrm{CO}_{2}$ and formate by a molybdenum-containing formate dehydrogenase. Journal of the American Chemical Society 136, 15473-15476.

Beaty, P.S., and Mcinerney, M.J. (1990). Nutritional features of Syntrophomonas wolfei. Applied and Environmental Microbiology 56, 3223-3224.

Bellack, A., Huber, H., Rachel, R., Wanner, G., and Wirth, R. (2011). Methanocaldococcus villosus sp. nov., a heavily flagellated archaeon that adheres to surfaces and forms cell-cell contacts. International Journal of Systematic and Evolutionary Microbiology $61,1239-1245$.

Belostotskiy, D.E., Ziganshina, E.E., Siniagina, M., Boulygina, E.A., Miluykov, V.A., and Ziganshin, A.M. (2015). Impact of the substrate loading regime and phosphoric acid supplementation on performance of biogas reactors and microbial community dynamics during anaerobic digestion of chicken wastes. Bioresource Technology $193,42-52$.

Bendtsen, J.D., Nielsen, H., Widdick, D., Palmer, T., and Brunak, S. (2005). Prediction of twin-arginine signal peptides. BMC Bioinformatics 6.

Berg, I.A., Kockelkorn, D., Ramos-Vera, W.H., Say, R.F., Zarzycki, J., Hugler, M., Alber, B.E., and Fuchs, G. (2010). Autotrophic carbon fixation in archaea. Nature Reviews Microbiology 8, 447-460. 
Berggren, G., Adamska, A., Lambertz, C., Simmons, T.R., Esselborn, J., Atta, M., Gambarelli, S., Mouesca, J.M., Reijerse, E., Lubitz, W., Happe, T., Artero, V., and Fontecave, M. (2013). Biomimetic assembly and activation of [FeFe]hydrogenases. Nature 499, 66-69.

Biegel, E., Schmidt, S., Gonzalez, J.M., and Müller, V. (2011). Biochemistry, evolution and physiological function of the Rnf complex, a novel ion-motive electron transport complex in prokaryotes. Cellular and molecular life sciences 68,613-634.

Blank, C.E. (2009). Not so old Archaea - the antiquity of biogeochemical processes in the archaeal domain of life. Geobiology 7, 495-514.

Boe, K., Steyer, J.P., and Angelidaki, I. (2008). Monitoring and control of the biogas process based on propionate concentration using online VFA measurement. Water Science and Technology 57, 661-666.

Bonacker, L.G., Baudner, S., and Thauer, R.K. (1992). Differential expression of the two methyl-coenzyme $\mathrm{M}$ reductases in Methanobacterium thermoautotrophicum as determined immunochemically via isoenzyme-specific antisera. European Journal of Biochemistry 206, 87-92.

Boone, D.R., and Bryant, M.P. (1980). Propionate-degrading bacterium, Syntrophobacter wolinii sp. nov. gen. nov., from methanogenic ecosystems. Applied and Environmental Microbiology 40, 626-632.

Boone, D.R., Johnson, R.L., and Liu, Y. (1989). Diffusion of the interspecies electron carriers $\mathrm{H}_{2}$ and formate in methanogenic ecosystems and its implications in the measurement of $\mathrm{K}_{\mathrm{m}}$ for $\mathrm{H}_{2}$ or formate uptake. Applied and Environmental Microbiology 55, 1735-1741.

Brileya, K.A., Camilleri, L.B., Zane, G.M., Wall, J.D., and Fields, M.W. (2014). Biofilm growth mode promotes maximum carrying capacity and community stability during product inhibition syntrophy. Frontiers in Microbiology 5, 693.

Brown, K.A., Wilker, M.B., Boehm, M., Dukovic, G., and King, P.W. (2012). Characterization of photochemical processes for $\mathrm{H}_{2}$ production by CdS nanorod-[FeFe] hydrogenase complexes. Journal of the American Chemical Society 134, 5627-5636.

Brutinel, E.D., and Gralnick, J.A. (2012). Shuttling happens: soluble flavin mediators of extracellular electron transfer in Shewanella. Applied Microbiology and Biotechnology 93, 41-48.

Bryant, M.P., and Boone, D.R. (1987). Isolation and characterization of Methanobacterium formicicum MF. International Journal of Systematic Bacteriology 37, 171-171.

Bryant, M.P., Wolin, E.A., Wolin, M.J., and Wolfe, R.S. (1967). Methanobacillus omelianskii, a symbiotic association of two species of bacteria. Arch Mikrobiol 59, 20-31.

Buckel, W., and Thauer, R.K. (2013). Energy conservation via electron bifurcating ferredoxin reduction and proton/ $\mathrm{Na}^{+}$translocating ferredoxin oxidation. Biochimica et Biophysica Acta 1827, 94-113.

Buyukkamaci, N., and Filibeli, A. (2004). Volatile fatty acid formation in an anaerobic hybrid reactor. Process Biochemistry 39, 1491-1494.

Calli, B., Mertoglu, B., Inanc, B., and Yenigun, O. (2005). Methanogenic diversity in anaerobic bioreactors under extremely high ammonia levels. Enzyme and Microbial Technology 37, 448-455.

Cammack, R. (1999). Hydrogenase sophistication. Nature 397, 214-215.

Candela, T., and Fouet, A. (2006). Poly-gamma-glutamate in bacteria. Molecular microbiology 60, 1091-1098.

Candela, T., Moya, M., Haustant, M., and Fouet, A. (2009). "Fusobacterium nucleatum, the first Gram-negative bacterium demonstrated to produce polyglutamate", in: Canadian journal of microbiology. 2009/06/02 ed.).

Caporaso, J.G., Kuczynski, J., Stombaugh, J., Bittinger, K., Bushman, F.D., Costello, E.K., Fierer, N., Pena, A.G., Goodrich, J.K., Gordon, J.I., Huttley, G.A., Kelley, S.T., Knights, D., Koenig, J.E., Ley, R.E., Lozupone, C.A., Mcdonald, D., Muegge, B.D., Pirrung, M., Reeder, J., Sevinsky, J.R., Tumbaugh, P.J., Walters, W.A., Widmann, J., Yatsunenko, T., Zaneveld, J., and Knight, R. (2010). QIIME allows analysis of high-throughput community sequencing data. Nature Methods 7, 335-336. 
Carballa, M., Regueiro, L., and Lema, J.M. (2015). Microbial management of anaerobic digestion: exploiting the microbiome-functionality nexus. Current Opinion in Biotechnology 33, 103-111.

Carbonero, F., Oakley, B.B., and Purdy, K.J. (2014). Metabolic flexibility as a major predictor of spatial distribution in microbial communities. PLoS One 9, e85105.

Castelle, C.J., Wrighton, K.C., Thomas, B.C., Hug, L.A., Brown, C.T., Wilkins, M.J., Frischkorn, K.R., Tringe, S.G., Singh, A., Markillie, L.M., Taylor, R.C., Williams, K.H., and Banfield, J.F. (2015). Genomic expansion of domain archaea highlights roles for organisms from new phyla in anaerobic carbon cycling. Current Biology 25, 690-701.

Ceccaldi, P., Marques, M.C., Fourmond, V., Pereira, I.C., and Leger, C. (2015). Oxidative inactivation of NiFeSe hydrogenase. Chemical Communications 51, 14223-14226.

Chabriere, E., Charon, M.H., Volbeda, A., Pieulle, L., Hatchikian, E.C., and Fontecilla-Camps, J.C. (1999). Crystal structures of the key anaerobic enzyme pyruvate:ferredoxin oxidoreductase, free and in complex with pyruvate. Nature structural biology 6 , 182-190.

Chang, Y.H., Han, J.I., Chun, J., Lee, K.C., Rhee, M.S., Kim, Y.B., and Bae, K.S. (2002). Comamonas koreensis sp. nov., a non-motile species from wetland in Woopo, Korea. International Journal of Systematic and Evolutionary Microbiology 52, 377381.

Chen, S., and Dong, X. (2005). Proteiniphilum acetatigenes gen. nov., sp. nov., from a UASB reactor treating brewery wastewater. International Journal of Systematic and Evolutionary Microbiology 55, 2257-2261.

Chen, S., Rotaru, A.E., Liu, F., Philips, J., Woodard, T.L., Nevin, K.P., and Lovley, D.R. (2014). Carbon cloth stimulates direct interspecies electron transfer in syntrophic co-cultures. Bioresource Technology 173, 82-86.

Chouari, R., Le Paslier, D., Daegelen, P., Ginestet, P., Weissenbach, J., and Sghir, A. (2005). Novel predominant archaeal and bacterial groups revealed by molecular analysis of an anaerobic sludge digester. Environmental Microbiology 7, 1104-1115.

Clark, M.E., He, Z., Redding, A.M., Joachimiak, M.P., Keasling, J.D., Zhou, J.Z., Arkin, A.P., Mukhopadhyay, A., and Fields, M.W. (2012). Transcriptomic and proteomic analyses of Desulfovibrio vulgaris biofilms: carbon and energy flow contribute to the distinct biofilm growth state. BMC Genomics 13, 138.

Cline, J.D. (1969). Spectrophotometric determination of hydrogen sulfide in natural waters. Limnology and Oceanography 14, 454-458.

Conrad, R. (1999). Contribution of hydrogen to methane production and control of hydrogen concentrations in methanogenic soils and sediments. FEMS Microbiology Ecology 28, 193-202.

Cord-Ruwisch, R., Mercz, T.I., Hoh, C.-Y., and Strong, G.E. (1997). Dissolved hydrogen concentration as an on-line control parameter for the automated operation and optimization of anaerobic digesters. Biotechnology and Bioengineering 56, 626634.

Costa, K.C., and Leigh, J.A. (2014). Metabolic versatility in methanogens. Current Opinion in Biotechnology 29, 70-75.

Costa, K.C., Lie, T.J., Jacobs, M.A., and Leigh, J.A. (2013a). $\mathrm{H}_{2}$-independent growth of the hydrogenotrophic methanogen Methanococcus maripaludis. MBio 4.

Costa, K.C., Lie, T.J., Xia, Q., and Leigh, J.A. (2013b). VhuD facilitates electron flow from $\mathrm{H}_{2}$ or formate to heterodisulfide reductase in Methanococcus maripaludis. Journal of Bacteriology 195, 5160-5165.

Costa, K.C., Wong, P.M., Wang, T., Lie, T.J., Dodsworth, J.A., Swanson, I., Burn, J.A., Hackett, M., and Leigh, J.A. (2010). Protein complexing in a methanogen suggests electron bifurcation and electron delivery from formate to heterodisulfide reductase. Proceedings of the National Academy of Sciences 107, 11050-11055.

Cruz Viggi, C., Rossetti, S., Fazi, S., Paiano, P., Majone, M., and Aulenta, F. (2014). Magnetite particles triggering a faster and more robust syntrophic pathway of methanogenic propionate degradation. Environmental Science \& Technology 48, 7536-7543. 
Cypionka, H. (1995). "Solute transport and cell energetics," ed. S. Us.), 151-184.

Da Silva, S.M., Pimentel, C., Valente, F.M., Rodrigues-Pousada, C., and Pereira, I.A. (2011). Tungsten and molybdenum regulation of formate dehydrogenase expression in Desulfovibrio vulgaris Hildenborough. Journal of Bacteriology 193, 2909-2916.

Da Silva, S.M., Voordouw, J., Leitao, C., Martins, M., Voordouw, G., and Pereira, I.A. (2013). Function of formate dehydrogenases in Desulfovibrio vulgaris Hildenborough energy metabolism. Microbiology 159, 1760-1769.

Daims, H., Brühl, A., Amann, R., Schleifer, K.-H., and Wagner, M. (1999). The domainspecific probe EUB338 is insufficient for the detection of all bacteria: Development and evaluation of a more comprehensive probe set. Systematic and Applied Microbiology 22, 434-444.

De Bok, F.a.M., Hagedoorn, P.-L., Silva, P.J., Hagen, W.R., Schiltz, E., Fritsche, K., and Stams, A.J.M. (2003). Two W-containing formate dehydrogenases $\left(\mathrm{CO}_{2-}\right.$ reductases) involved in syntrophic propionate oxidation by Syntrophobacter fumaroxidans. European Journal of Biochemistry 270, 2476-2485.

De Bok, F.a.M., Harmsen, H.J.M., Plugge, C.M., De Vries, M.C., Akkermans, A.D.L., De Vos, W.M., and Stams, A.J.M. (2005). The first true obligately syntrophic propionateoxidizing bacterium, Pelotomaculum schinkii sp. nov., co-cultured with Methanospirillum hungatei, and emended description of the genus Pelotomaculum. International Journal of Systematic and Evolutionary Microbiology 55, 1697-1703.

De Bok, F.a.M., Luijten, M.L.G.C., and Stams, A.J.M. (2002a). Biochemical evidence for formate transfer in syntrophic propionate-oxidizing cocultures of Syntrophobacter fumaroxidans and Methanospirillum hungatei. Applied and Environmental Microbiology 68, 4247-4252.

De Bok, F.a.M., Roze, E.H., and Stams, A.J.M. (2002b). Hydrogenases and formate dehydrogenases of Syntrophobacter fumaroxidans. Antonie van Leeuwenhoek 81, 283-291.

De Bok, F.a.M., Stams, A.J.M., Dijkema, C., and Boone, D.R. (2001). Pathway of propionate oxidation by a syntrophic culture of Smithella propionica and Methanospirillum hungatei. Applied and Environmental Microbiology 67, 1800-1804.

De Vrieze, J., Gildemyn, S., Vilchez-Vargas, R., Jáuregui, R., Pieper, D.H., Verstraete, W., and Boon, N. (2015). Inoculum selection is crucial to ensure operational stability in anaerobic digestion. Applied Microbiology and Biotechnology 99, 189-199.

Dereli, R.K., Grelot, A., Heffernan, B., Van Der Zee, F.P., and Van Lier, J.B. (2014). Implications of changes in solids retention time on long term evolution of sludge filterability in anaerobic membrane bioreactors treating high strength industrial wastewater. Water Research 59, 11-22.

Dereli, R.K., Heffernan, B., Grelot, A., Van Der Zee, F.P., and Van Lier, J.B. (2015). Influence of high lipid containing wastewater on filtration performance and fouling in AnMBRs operated at different solids retention times. Separation and Purification Technology $139,43-52$

Diender, M., Pereira, R., Wessels, H.J., Stams, A.J.M., and Sousa, D.Z. (2016). Proteomic analysis of the hydrogen and carbon monoxide metabolism of Methanothermobacter marburgensis. Frontiers in Microbiology 7, 1049.

Dolfing, J., Jiang, B., Henstra, A.M., Stams, A.J.M., and Plugge, C.M. (2008). Syntrophic growth on formate: a new microbial niche in anoxic environments. Applied and Environmental Microbiology 74, 6126-6131.

Dong, X., Plugge, C.M., and Stams, A.J.M. (1994). Anaerobic degradation of propionate by a mesophilic acetogenic bacterium in coculture and triculture with different methanogens. Applied and Environmental Microbiology 60, 2834-2838.

Dong, X., and Stams, A.J.M. (1995). Evidence for $\mathrm{H}_{2}$ and formate formation during syntrophic butyrate and propionate degradation. Anaerobe 1, 35-39.

Duarte, A.G., Santos, A.A., and Pereira, I.A. (2016). Electron transfer between the QmoABC membrane complex and adenosine 5'-phosphosulfate reductase. Biochimica et Biophysica Acta 1857, 380-386. 
El-Zahab, B., Donnelly, D., and Wang, P. (2008). Particle-tethered NADH for production of methanol from $\mathrm{CO}_{2}$ catalyzed by coimmobilized enzymes. Biotechnology and Bioengineering 99, 508-514.

Esquivel, R.N., Xu, R., and Pohlschroder, M. (2013). Novel archaeal adhesion pilins with a conserved N terminus. Journal of Bacteriology 195, 3808-3818.

Fang, H.H.P.C., H. K., Li, Y.Y., and Chen, T. (1994). Performance and granule characteristics of UASB process treating wastewater with hydrolyzed proteins. Water Science and Technology 30, 55-63.

Ferry, J.G. (1990). Formate dehydrogenase. FEMS Microbiology Reviews 7, 377-382.

Ferry, J.G., and Lessner, D.J. (2008). Methanogenesis in marine sediments. Annals of the New York Academy of Sciences 1125, 147-157.

Ferry, J.G., and Wolfe, R.S. (1976). Anaerobic degradation of benzoate to methane by a microbial consortium. Archives of Microbiology 107, 33-40.

Ferry, J.G., and Wolfe, R.S. (1977). Nutritional and biochemical characterization of Methanospirillum hungatii. Applied and Environmental Microbiology 34, 371-376.

Fotidis, I.A., Karakashev, D., and Angelidaki, I. (2013). The dominant acetate degradation pathway/methanogenic composition in full-scale anaerobic digesters operating under different ammonia levels. International Journal of Environmental Science and Technology 11, 2087-2094.

Franke-Whittle, I.H., Walter, A., Ebner, C., and Insam, H. (2014). Investigation into the effect of high concentrations of volatile fatty acids in anaerobic digestion on methanogenic communities. Waste Management 34, 2080-2089.

Garcia Costas, A.M., Poudel, S., Miller, A.F., Schut, G.J., Ledbetter, R.N., Fixen, K.R., Seefeldt, L.C., Adams, M.W.W., Harwood, C.S., Boyd, E.S., and Peters, J.W. (2017). Defining electron bifurcation in the electron-transferring flavoprotein family. Journal of Bacteriology 199.

Garcin, E., Vernede, X., Hatchikian, E.C., Volbeda, A., Frey, M., and Fontecilla-Camps, J.C. (1999). The crystal structure of a reduced [NiFeSe] hydrogenase provides an image of the activated catalytic center. Structure 7, 557-566.

Ge, H., Batstone, D.J., and Keller, J. (2015). Biological phosphorus removal from abattoir wastewater at very short sludge ages mediated by novel PAO clade Comamonadaceae. Water Research 69, 173-182.

Giovannini, G., Donoso-Bravo, A., Jeison, D., Chamy, R., Ruiz-Filippi, G., and Vande Wouwer, A. (2016). A review of the role of hydrogen in past and current modelling approaches to anaerobic digestion processes. International Journal of Hydrogen Energy 41, 17713-17722.

Goodwin, J.a.S., Finlayson, J.M., and Low, E.W. (2001). A further study of the anaerobic biotreatment of malt whisky distillery pot ale using an UASB system. Bioresource Technology 78, 155-160.

Goodwin, J.a.S., and Stuart, J.B. (1994). Anaerobic digestion of malt whisky distillery pot ale using upflow anaerobic sludge blanket reactors. Bioresource Technology 49, $75-$ 81.

Gorby, Y.A., Yanina, S., Mclean, J.S., Rosso, K.M., Moyles, D., Dohnalkova, A., Beveridge, T.J., Chang, I.S., Kim, B.H., Kim, K.S., Culley, D.E., Reed, S.B., Romine, M.F., Saffarini, D.A., Hill, E.A., Shi, L., Elias, D.A., Kennedy, D.W., Pinchuk, G., Watanabe, K., Ishii, S., Logan, B., Nealson, K.H., and Fredrickson, J.K. (2006). Electrically conductive bacterial nanowires produced by Shewanella oneidensis strain MR-1 and other microorganisms. Proceedings of the National Academy of Sciences 103, 11358-11363.

Grabowski, A., Tindall, B.J., Bardin, V., Blanchet, D., and Jeanthon, C. (2005). Petrimonas sulfuriphila gen. nov., sp. nov., a mesophilic fermentative bacterium isolated from a biodegraded oil reservoir. International Journal of Systematic and Evolutionary Microbiology 55, 1113-1121.

Graham, J., Peter, B., Walker, G.M., Wardlaw, A., and Campbell, E. (2012). "Characterisation of the pot ale profile from a malt whisky distillery," in Distilled Spirits: Science and Sustainability.). 
Grein, F., Ramos, A.R., Venceslau, S.S., and Pereira, I.A. (2013). Unifying concepts in anaerobic respiration: insights from dissimilatory sulfur metabolism. Biochimica et Biophysica Acta 1827, 145-160.

Grosskopf, T., Zenobi, S., Alston, M., Folkes, L., Swarbreck, D., and Soyer, O.S. (2016). A stable genetic polymorphism underpinning microbial syntrophy. The ISME Journal $10,2844-2853$.

Gunsalus, R.P., Cook, L.E., Crable, B., Rohlin, L., Mcdonald, E., Mouttaki, H., Sieber, J.R., Poweleit, N., Zhou, H., Lapidus, A.L., Daligault, H.E., Land, M., Gilna, P., Ivanova, N., Kyrpides, N., Culley, D.E., and Mcinerney, M.J. (2016). Complete genome sequence of Methanospirillum hungatei type strain JF1. Standards in Genomic Sciences 11, 2.

Guo, X.H., Wang, C., Sun, F.Q., Zhu, W.J., and Wu, W.X. (2014). A comparison of microbial characteristics between the thermophilic and mesophilic anaerobic digesters exposed to elevated food waste loadings. Bioresource Technology 152, 420-428.

Guwy, A.J., Hawkes, F.R., Hawkes, D.L., and Rozzi, A.G. (1997). Hydrogen production in a high rate fluidised bed anaerobic digester. Water Research 31, 1291-1298.

Hahnke, S., Langer, T., Koeck, D.E., and Klocke, M. (2016). Description of Proteiniphilum saccharofermentans sp. nov., Petrimonas mucosa sp. nov. and Fermentimonas caenicola gen. nov., sp. nov. isolated from mesophilic lab-scale biogas reactors and emended description of the genus Proteiniphilum. International Journal of Systematic and Evolutionary Microbiology.

Hahnke, S., Maus, I., Wibberg, D., Tomazetto, G., Puhler, A., Klocke, M., and Schluter, A. (2015). Complete genome sequence of the novel Porphyromonadaceae bacterium strain ING2-E5B isolated from a mesophilic lab-scale biogas reactor. Journal of Biotechnology 193, 34-36.

Hamady, M., Walker, J.J., Harris, J.K., Gold, N.J., and Knight, R. (2008). Error-correcting barcoded primers for pyrosequencing hundreds of samples in multiplex. Nature Methods 5, 235-237.

Hamann, N., Mander, G.J., Shokes, J.E., Scott, R.A., Bennati, M., and Hedderich, R. (2007). A cysteine-rich CCG domain contains a novel [4Fe-4S] cluster binding motif as deduced from studies with subunit $B$ of heterodisulfide reductase from Methanothermobacter marburgensis. Biochemistry 46, 12875-12885.

Hambourger, M., Gervaldo, M., Svedruzic, D., King, P.W., Gust, D., Ghirardi, M., Moore, A.L., and Moore, T.A. (2008). [FeFe]-hydrogenase-catalyzed $\mathrm{H}_{2}$ production in a photoelectrochemical biofuel cell. Journal of the American Chemical Society 130, 2015-2022.

Hansen, T.A. (1994). Metabolism of sulfate-reducing prokaryotes. Antonie Van Leeuwenhoek International Journal of General and Molecular Microbiology 66, 165-185.

Harmsen, H.J.M., Van Kuijk, B.L.M., Plugge, C.M., Akkermans, A.D.L., De Vos, W.M., and Stams, A.J.M. (1998). Syntrophobacter fumaroxidans sp. nov., a syntrophic propionate-degrading sulfate-reducing bacterium. International Journal of Systematic Bacteriology 48 Pt 4, 1383-1387.

Hattori, S. (2008). Syntrophic acetate-oxidizing microbes in methanogenic environments. Microbes and environments 23, 118-127.

He, Y., Xu, P., Li, C., and Zhang, B. (2005). High-concentration food wastewater treatment by an anaerobic membrane bioreactor. Water Research 39, 4110-4118.

Hedderich, R., and Forzi, L. (2005). Energy-converting [NiFe] hydrogenases: more than just $\mathrm{H}_{2}$ activation. Journal of Molecular Microbiology and Biotechnology 10, 92-104.

Hedderich, R., Hamann, N., and Bennati, M. (2005). Heterodisulfide reductase from methanogenic archaea: a new catalytic role for an iron-sulfur cluster. Biological Chemistry 386, 961-970.

Hedderich, R., Koch, J., Linder, D., and Thauer, R.K. (1994). The heterodisulfide reductase from Methanobacterium thermoautotrophicum contains sequence motifs characteristic of pyridine-nucleotide-dependent thioredoxin reductases. European Journal of Biochemistry 225, 253-261. 
Hemmingsen, S.M., Woolford, C., Vandervies, S.M., Tilly, K., Dennis, D.T., Georgopoulos, C.P., Hendrix, R.W., and Ellis, R.J. (1988). Homologous plant and bacterial proteins chaperone oligomeric protein assembly. Nature 333, 330-334.

Herrmann, G., Jayamani, E., Mai, G., and Buckel, W. (2008). Energy conservation via electron-transferring flavoprotein in anaerobic bacteria. Journal of Bacteriology 190, 784-791.

Hillesland, K.L., Lim, S., Flowers, J.J., Turkarslan, S., Pinel, N., Zane, G.M., Elliott, N., Qin, Y., Wu, L., Baliga, N.S., Zhou, J., Wall, J.D., and Stahl, D.A. (2014). Erosion of functional independence early in the evolution of a microbial mutualism. Proceedings of the National Academy of Sciences 111, 14822-14827.

Hillesland, K.L., and Stahl, D.A. (2010). Rapid evolution of stability and productivity at the origin of a microbial mutualism. Proceedings of the National Academy of Sciences $107,2124-2129$.

Hoben, J.P., Lubner, C.E., Ratzloff, M.W., Schut, G.J., Nguyen, D.M.N., Hempel, K.W., Adams, M.W.W., King, P.W., and Miller, A.F. (2017). Equilibrium and ultrafast kinetic studies manipulating electron transfer: A short-lived flavin semiquinone is not sufficient for electron bifurcation. Journal of Biological Chemistry 292, 1403914049.

Hochheimer, A., Hedderich, R., and Thauer, R.K. (1998). The formylmethanofuran dehydrogenase isoenzymes in Methanobacterium wolfei and Methanobacterium thermoautotrophicum: induction of the molybdenum isoenzyme by molybdate and constitutive synthesis of the tungsten isoenzyme. Archives of Microbiology 170, 389-393.

Hochheimer, A., Linder, D., Thauer, R.K., and Hedderich, R. (1996). The molybdenum formylmethanofuran dehydrogenase operon and the tungsten formylmethanofuran dehydrogenase operon from Methanobacterium thermoautotrophicum. Structures and transcriptional regulation. European Journal of Biochemistry 242, 156-162.

Hochheimer, A., Schmitz, R.A., Thauer, R.K., and Hedderich, R. (1995). The tungsten formylmethanofuran dehydrogenase from Methanobacterium thermoautotrophicum contains sequence motifs characteristic for enzymes containing molybdopterin dinucleotide. European Journal of Biochemistry 234, 910920.

Holmes, D.E., Shrestha, P.M., Walker, D.J., Dang, Y., Nevin, K.P., Woodard, T.L., and Lovley, D.R. (2017). Metatranscriptomic evidence for direct interspecies electron transfer between Geobacter and Methanothrix species in methanogenic rice paddy soils. Applied and Environmental Microbiology 83, e00223-00217.

Huang, H., Wang, S., Moll, J., and Thauer, R.K. (2012). Electron bifurcation involved in the energy metabolism of the acetogenic bacterium Moorella thermoacetica growing on glucose or $\mathrm{H}_{2}$ plus $\mathrm{CO}_{2}$. Journal of Bacteriology 194, 3689-3699.

Hug, L.A., Baker, B.J., Anantharaman, K., Brown, C.T., Probst, A.J., Castelle, C.J., Butterfield, C.N., Hernsdorf, A.W., Amano, Y., Ise, K., Suzuki, Y., Dudek, N., Relman, D.A., Finstad, K.M., Amundson, R., Thomas, B.C., and Banfield, J.F. (2016). A new view of the tree of life. Nat Microbiol 1, 16048.

Ikeda, M., Sato, T., Wachi, M., Jung, H.K., Ishino, F., Kobayashi, Y., and Matsuhashi, M. (1989). Structural similarity among Escherichia coli FtsW and RodA proteins and Bacillus subtilis SpoVE protein, which function in cell division, cell elongation, and spore formation, respectively. Journal of Bacteriology 171, 6375-6378.

Imachi, H., Sakai, S., Ohashi, A., Harada, H., Hanada, S., Kamagata, Y., and Sekiguchi, Y. (2007). Pelotomaculum propionicicum sp. nov., an anaerobic, mesophilic, obligately syntrophic, propionate-oxidizing bacterium. International Journal of Systematic and Evolutionary Microbiology 57, 1487-1492.

Imachi, H., Sekiguchi, Y., Kamagata, Y., Hanada, S., Ohashi, A., and Harada, H. (2002). Pelotomaculum thermopropionicum gen. nov., sp. nov., an anaerobic, thermophilic, syntrophic propionate-oxidizing bacterium. International journal of systematic and evolutionary microbiology 52, 1729-1735.

Imachi, H., Sekiguchi, Y., Kamagata, Y., Loy, A., Qiu, Y.L., Hugenholtz, P., Kimura, N., Wagner, M., Ohashi, A., and Harada, H. (2006). Non-sulfate-reducing, syntrophic 
bacteria affiliated with Desulfotomaculum cluster I are widely distributed in methanogenic environments. Applied and Environmental Microbiology 72, 20802091.

Ishihama, Y., Schmidt, T., Rappsilber, J., Mann, M., Hartl, F.U., Kerner, M.J., and Frishman, D. (2008). Protein abundance profiling of the Escherichia coli cytosol. BMC Genomics 9, 102.

Ishii, S., Kosaka, T., Hori, K., Hotta, Y., and Watanabe, K. (2005). Coaggregation facilitates interspecies hydrogen transfer between Pelotomaculum thermopropionicum and Methanothermobacter thermautotrophicus. Applied and Environmental Microbiology 71, 7838-7845.

Jackson, B.E., Bhupathiraju, V.K., Tanner, R.S., Woese, C.R., and Mcinerney, M.J. (1999). Syntrophus aciditrophicus sp. nov., a new anaerobic bacterium that degrades fatty acids and benzoate in syntrophic association with hydrogen-using microorganisms. Archives of microbiology 171, 107-114.

Jarrell, K.F., and Albers, S.V. (2012). The archaellum: an old motility structure with a new name. Trends in Microbiology 20, 307-312.

Jarrell, K.F., Stark, M., Nair, D.B., and Chong, J.P.J. (2011). Flagella and pili are both necessary for efficient attachment of Methanococcus maripaludis to surfaces. FEMS Microbiology Letters 319, 44-50.

Jing, H., Takagi, J., Liu, J.H., Lindgren, S., Zhang, R.G., Joachimiak, A., Wang, J.H., and Springer, T.A. (2002). Archaeal surface layer proteins contain beta propeller, PKD, and beta helix domains and are related to metazoan cell surface proteins. Structure $10,1453-1464$.

Joris, B., Dive, G., Henriques, A., Piggot, P.J., and Ghuysen, J.M. (1990). The life-cycle proteins RodA of Escherichia coli and SpoVE of Bacillus subtilis have very similar primary structures. Mol Microbiol 4, 513-517.

Junicke, H., Abbas, B., Oentoro, J., Van Loosdrecht, M., and Kleerebezem, R. (2014). Absolute quantification of individual biomass concentrations in a methanogenic coculture. AMB Express 4.

Junicke, H., Feldman, H., Van Loosdrecht, M.C., and Kleerebezem, R. (2015). Impact of the hydrogen partial pressure on lactate degradation in a coculture of Desulfovibrio sp. G11 and Methanobrevibacter arboriphilus DH1. Applied Microbiology and Biotechnology 99, 3599-3608.

Kaden, J., A, S.G., and Schink, B. (2002). Cysteine-mediated electron transfer in syntrophic acetate oxidation by cocultures of Geobacter sulfurreducens and Wolinella succinogenes. Archives of Microbiology 178, 53-58.

Kaster, A.K., Goenrich, M., Seedorf, H., Liesegang, H., Wollherr, A., Gottschalk, G., and Thauer, R.K. (2011a). More than 200 genes required for methane formation from $\mathrm{H}(2)$ and $\mathrm{CO}(2)$ and energy conservation are present in Methanothermobacter marburgensis and Methanothermobacter thermautotrophicus. Archaea 2011, 973848.

Kaster, A.K., Moll, J., Parey, K., and Thauer, R.K. (2011b). Coupling of ferredoxin and heterodisulfide reduction via electron bifurcation in hydrogenotrophic methanogenic archaea. Proceedings of the National Academy of Sciences 108, 2981-2986.

Kato, S. (2015). Biotechnological aspects of microbial extracellular electron transfer. Microbes and Environments 30, 133-139.

Kato, S., and Watanabe, K. (2010). Ecological and evolutionary interactions in syntrophic methanogenic consortia. Microbes and Environments 25, 145-151.

Kelly, W.J., Leahy, S.C., Li, D., Perry, R., Lambie, S.C., Attwood, G.T., and Altermann, E. (2014). The complete genome sequence of the rumen methanogen Methanobacterium formicicum BRM9. Standards in Genomic Sciences 9, 15.

Khan, S.T., Horiba, Y., Yamamoto, M., and Hiraishi, A. (2002). Members of the family Comamonadaceae as primary poly(3-hydroxybutyrate-co-3-hydroxyvalerate)degrading denitrifiers in activated sludge as revealed by a polyphasic approach. Applied and Environmental Microbiology 68, 3206-3214. 
Kidby, D.W., and Nedwell, D.B. (1991). An Investigation into the Suitability of Biogas Hydrogen Concentration as a Performance Monitor for Anaerobic Sewage-Sludge Digesters. Water Research 25, 1007-1012.

Kleerebezem, R., and Stams, A.J.M. (2000). Kinetics of syntrophic cultures: a theoretical treatise on butyrate fermentation. Biotechnology and Bioengineering 67, 529-543.

Klein, M., Friedrich, M., Roger, A.J., Hugenholtz, P., Fishbain, S., Abicht, H., Blackall, L.L., Stahl, D.A., and Wagner, M. (2001). Multiple lateral transfers of dissimilatory sulfite reductase genes between major lineages of sulfate-reducing prokaryotes. Journal of Bacteriology 183, 6028-6035.

Kosaka, T., Kato, S., Shimoyama, T., Ishii, S., Abe, T., and Watanabe, K. (2008). The genome of Pelotomaculum thermopropionicum reveals niche-associated evolution in anaerobic microbiota. Genome Research 18, 442-448.

Kosaka, T., Uchiyama, T., Ishii, S., Enoki, M., Imachi, H., Kamagata, Y., Ohashi, A., Harada, H., Ikenaga, H., and Watanabe, K. (2006). Reconstruction and regulation of the central catabolic pathway in the thermophilic propionate-oxidizing syntroph Pelotomaculum thermopropionicum. Journal of Bacteriology 188, 202-210.

Koster, I.W., and Lettinga, G. (1984). The influence of ammonium-nitrogen on the specific activity of pelletized methanogenic sludge. Agricultural Wastes 9, 205-216.

Kristensen, E., Ahmed, S.I., and Devol, A.H. (1995). Aerobic and anaerobic decomposition of organic matter in marine sediment: Which is fastest? Limnology and Oceanography 40, 1430-1437.

Kristjansson, J.K., Schönheit, P., and Thauer, R.K. (1982). Different Ks values for hydrogen of methanogenic bacteria and sulfate reducing bacteria: an explanation for the apparent inhibition of methanogenesis by sulfate. Archives of Microbiology 131, 278-282.

Krogh, A., Larsson, B., Von Heijne, G., and Sonnhammer, E.L.L. (2001). Predicting transmembrane protein topology with a hidden Markov model: Application to complete genomes. Journal of Molecular Biology 305, 567-580.

Krumholz, L.R., Bradstock, P., Sheik, C.S., Diao, Y., Gazioglu, O., Gorby, Y., and Mcinerney, M.J. (2015). Syntrophic growth of Desulfovibrio alaskensis requires genes for $\mathrm{H}_{2}$ and formate metabolism as well as those for flagellum and biofilm formation. Applied and Environmental Microbiology 81, 2339-2348.

Kvist, T., Ahring, B.K., and Westermann, P. (2007). Archaeal diversity in Icelandic hot springs. FEMS Microbiology Ecology 59, 71-80.

Laanbroek, H.J., Abee, T., and Voogd, I.L. (1982). Alcohol conversions by Desulfobulbus propionicus Lindhorst in the presence and absence of sulfate and hydrogen. Archives of Microbiology 133, 178-184.

Larsen, S., Nielsen, L.P., and Schramm, A. (2015). Cable bacteria associated with longdistance electron transport in New England salt marsh sediment. Environmental Microbiology Reports 7, 175-179.

Le-Clech, P. (2010). Membrane bioreactors and their uses in wastewater treatments. Applied microbiology and biotechnology 88, 1253-1260.

Lee, S.H., Park, J.H., Kim, S.H., Yu, B.J., Yoon, J.J., and Park, H.D. (2015). Evidence of syntrophic acetate oxidation by Spirochaetes during anaerobic methane production. Bioresource Technology 190, 543-549.

Lens, P.N.L., Visser, A., Janssen, A.J.H., Hulshoff Pol, L.W., and Lettinga, G. (1998). Biotechnological treatment of sulfate-rich wastewaters. Critical Reviews in Environmental Science and Technology 28, 41-88.

Lenz, O., Ludwig, M., Schubert, T., Bürstel, I., Ganskow, S., Goris, T., Schwarze, A., and Friedrich, B. (2010). $\mathrm{H}_{2}$ conversion in the presence of $\mathrm{O}_{2}$ as performed by the membrane-bound [NiFe]-hydrogenase of Ralstonia eutropha. ChemPhysChem 11, 1107-1119.

Li, F., Hinderberger, J., Seedorf, H., Zhang, J., Buckel, W., and Thauer, R.K. (2008). Coupled ferredoxin and crotonyl coenzyme A (CoA) reduction with NADH catalyzed by the butyryl-CoA dehydrogenase/Etf complex from Clostridium kluyveri. Journal of Bacteriology 190, 843-850. 
Li, L., He, Q., Ma, Y., Wang, X., and Peng, X. (2016). A mesophilic anaerobic digester for treating food waste: process stability and microbial community analysis using pyrosequencing. Microbial Cell Factories 15, 65.

Li, L., He, Q., Ma, Y., Wang, X.M., and Peng, X.Y. (2015). Dynamics of microbial community in a mesophilic anaerobic digester treating food waste: Relationship between community structure and process stability. Bioresource Technology 189, 113-120.

Li, X., Luo, Q., Wofford, N.Q., Keller, K.L., Mcinerney, M.J., Wall, J.D., and Krumholz, L.R. (2009). A molybdopterin oxidoreductase is involved in $\mathrm{H} 2$ oxidation in Desulfovibrio desulfuricans G20. Journal of Bacteriology 191, 2675-2682.

Li, X., Mcinerney, M.J., Stahl, D.A., and Krumholz, L.R. (2011). Metabolism of $\mathrm{H}_{2}$ by Desulfovibrio alaskensis G20 during syntrophic growth on lactate. Microbiology 157, 2912-2921.

Liao, B.Q., Kraemer, J.T., and Bagley, D.M. (2006). Anaerobic membrane bioreactors: Applications and research directions. Critical Reviews in Environmental Science and Technology 36, 489-530.

Lie, T.J., Costa, K.C., Lupa, B., Korpole, S., Whitman, W.B., and Leigh, J.A. (2012). Essential anaplerotic role for the energy-converting hydrogenase Eha in hydrogenotrophic methanogenesis. Proceedings of the National Academy of Sciences 109, 1547315478.

Lin, H., Peng, W., Zhang, M., Chen, J., Hong, H., and Zhang, Y. (2013). A review on anaerobic membrane bioreactors: Applications, membrane fouling and future perspectives. Desalination 314, 169-188.

Liu, F., Rotaru, A.-E., Shrestha, P.M., Malvankar, N.S., Nevin, K.P., and Lovley, D.R. (2012). Promoting direct interspecies electron transfer with activated carbon. Energy \& Environmental Science 5, 8982.

Liu, P., and Conrad, R. (2017). Syntrophobacteraceae-affiliated species are major propionate-degrading sulfate reducers in paddy soil. Environmental Microbiology 19, 1669-1686.

Liu, Y., Balkwill, D.L., Aldrich, H.C., Drake, G.R., and Boone, D.R. (1999). Characterization of the anaerobic propionate-degrading syntrophs Smithella propionica gen. nov., sp. nov. and Syntrophobacter wolinii. International Journal of Systematic Bacteriology 49 Pt 2, 545-556.

Liu, Y., and Whitman, W.B. (2008). Metabolic, phylogenetic, and ecological diversity of the methanogenic archaea. Annals of the New York Academy of Sciences 1125, 171189.

Losey, N.A., Mus, F., Peters, J.W., Le, H.M., and Mcinerney, M.J. (2017). Syntrophomonas wolfei uses an NADH-dependent, ferredoxin-independent [FeFe]-hydrogenase to reoxidize NADH. Applied Environmental Microbiology 83.

Lovley, D.R. (1985). Minimum threshold for hydrogen metabolism in methanogenic bacteria. Applied and Environmental Microbiology 49, 1530-1531.

Lovley, D.R. (2017). Syntrophy goes electric: Direct Interspecies Electron Transfer. Annual Review of Microbiology 71, 643-664.

Lovley, D.R., Fraga, J.L., Coates, J.D., and Blunt-Harris, E.L. (1999). Humics as an electron donor for anaerobic respiration. Environmental Microbiology 1, 89-98.

Lovley, D.R., and Klug, M.J. (1983). Sulfate reducers can outcompete methanogens at freshwater sulfate concentrations. Applied and Environmental Microbiology 45, 187-192.

Lovley, D.R., and Malvankar, N.S. (2015). Seeing is believing: novel imaging techniques help clarify microbial nanowire structure and function. Environmental Microbiology 17, 2209-2215.

Lu, J., Boeren, S., De Vries, S.C., Van Valenberg, H.J.F., Vervoort, J., and Hettinga, K. (2011). Filter-aided sample preparation with dimethyl labeling to identify and quantify milk fat globule membrane proteins. Journal of Proteomics 75, 34-43.

Lu, P., Vogel, C., Wang, R., Yao, X., and Marcotte, E.M. (2007). Absolute protein expression profiling estimates the relative contributions of transcriptional and translational regulation. Nature Biotechnology 25, 117-124. 
Lubitz, W., Ogata, H., Rudiger, O., and Reijerse, E. (2014). Hydrogenases. Chemical Reviews $114,4081-4148$.

Lubner, C.E., Jennings, D.P., Mulder, D.W., Schut, G.J., Zadvornyy, O.A., Hoben, J.P., Tokmina-Lukaszewska, M., Berry, L., Nguyen, D.M., Lipscomb, G.L., Bothner, B., Jones, A.K., Miller, A.F., King, P.W., Adams, M.W.W., and Peters, J.W. (2017). Mechanistic insights into energy conservation by flavin-based electron bifurcation. Nature Chemical Biology 13, 655-659.

Lucas, R., Kuchenbuch, A., Fetzer, I., Harms, H., and Kleinsteuber, S. (2015). Long-term monitoring reveals stable and remarkably similar microbial communities in parallel full-scale biogas reactors digesting energy crops. FEMS Microbiology Ecology 91.

Lupa, B., Hendrickson, E.L., Leigh, J.A., and Whitman, W.B. (2008). Formate-dependent $\mathrm{H}_{2}$ production by the mesophilic methanogen Methanococcus maripaludis. Applied and Environmental Microbiology 74, 6584-6590.

Luther, G.W., 3rd, Findlay, A.J., Macdonald, D.J., Owings, S.M., Hanson, T.E., Beinart, R.A., and Girguis, P.R. (2011). Thermodynamics and kinetics of sulfide oxidation by oxygen: a look at inorganically controlled reactions and biologically mediated processes in the environment. Frontiers in microbiology 2, 62.

Lykidis, A., Chen, C.L., Tringe, S.G., Mchardy, A.C., Copeland, A., Kyrpides, N.C., Hugenholtz, P., Macarie, H., Olmos, A., Monroy, O., and Liu, W.T. (2011). Multiple syntrophic interactions in a terephthalate-degrading methanogenic consortium. The ISME Journal 5, 122-130.

Mancuso, F., Bunkenborg, J., Wierer, M., and Molina, H. (2012). Data extraction from proteomics raw data: an evaluation of nine tandem MS tools using a large Orbitrap data set. Journal of Proteomics 75, 5293-5303.

Mander, G.J., Pierik, A.J., Huber, H., and Hedderich, R. (2004). Two distinct heterodisulfide reductase-like enzymes in the sulfate-reducing archaeon Archaeoglobus profundus. European Journal of Biochemistry 271, 1106-1116.

Marchaim, U., and Krause, C. (1993). Propionic to Acetic-Acid Ratios in Overloaded Anaerobic-Digestion. Bioresource Technology 43, 195-203.

Markowitz, V.M., Chen, I.-M.A., Palaniappan, K., Chu, K., Szeto, E., Grechkin, Y., Ratner, A., Jacob, B., Huang, J., Williams, P., Huntemann, M., Anderson, I., Mavromatis, K., Ivanova, N.N., and Kyrpides, N.C. (Version 4.2. November 2013). IMG: the integrated microbial genomes database and comparative analysis system DOE-JGI, Walnut Creek, USA.

Marques, M.C., Tapia, C., Gutierrez-Sanz, O., Ramos, A.R., Keller, K.L., Wall, J.D., De Lacey, A.L., Matias, P.M., and Pereira, I.a.C. (2017). The direct role of selenocysteine in [NiFeSe] hydrogenase maturation and catalysis. Nat Chem Biol 13, 544-550.

Martins, M., Mourato, C., Morais-Silva, F.O., Rodrigues-Pousada, C., Voordouw, G., Wall, J.D., and Pereira, I.A. (2016). Electron transfer pathways of formate-driven $\mathrm{H}_{2}$ production in Desulfovibrio. Applied Microbiology and Biotechnology 100, 81358146.

Martins, M., Mourato, C., and Pereira, I.A. (2015). Desulfovibrio vulgaris growth coupled to formate-driven $\mathrm{H}_{2}$ production. Environmental Science \& Technology 49, 1465514662.

Mathews, D.H., Sabina, J., Zuker, M., and Turner, D.H. (1999). Expanded sequence dependence of thermodynamic parameters improves prediction of RNA secondary structure. Journal of Molecular Biology 288, 911-940.

Matschiavelli, N., and Rother, M. (2015). Role of a putative tungsten-dependent formylmethanofuran dehydrogenase in Methanosarcina acetivorans. Archives of Microbiology 197, 379-388.

Mattevi, A., Tedeschi, G., Bacchella, L., Coda, A., Negri, A., and Ronchi, S. (1999). Structure of L-aspartate oxidase: implications for the succinate dehydrogenase/fumarate reductase oxidoreductase family. Structure 7, 745-756.

Maus, I., Stantscheff, R., Wibberg, D., Stolze, Y., Winkler, A., Puhler, A., Konig, H., and Schluter, A. (2014). Complete genome sequence of the methanogenic neotype strain Methanobacterium formicicum MF'. Journal of Biotechnology 192 Pt A, 4041. 
May, H.D., Patel, P.S., and Ferry, J.G. (1988). Effect of molybdenum and tungsten on synthesis and composition of formate dehydrogenase in Methanobacterium formicicum. Journal of Bacteriology 170, 3384-3389.

Mccarthy, P. (1964). Anaerobic waste treatment fundamentals. Part III: toxic materials and their control. Public works 95, 91-94.

Mcinerney, M.J., Bryant, M.P., Hespell, R.B., and Costerton, J.W. (1981). Syntrophomonas wolfei gen. nov. sp. nov., an anaerobic, syntrophic, fatty acid-oxidizing bacterium. Applied and Environmental Microbiology 41, 1029-1039.

Mcinerney, M.J., Bryant, M.P., and Pfennig, N. (1979). Anaerobic bacterium that degrades fatty-acids in syntrophic association with methanogens. Archives of Microbiology $122,129-135$.

Mcinerney, M.J., Rohlin, L., Mouttaki, H., Kim, U., Krupp, R.S., Rios-Hernandez, L., Sieber, J., Struchtemeyer, C.G., Bhattacharyya, A., Campbell, J.W., and Gunsalus, R.P. (2007). The genome of Syntrophus aciditrophicus: life at the thermodynamic limit of microbial growth. Proceedings of the National Academy of Sciences 104, 76007605.

Mcinerney, M.J., Sieber, J.R., and Gunsalus, R.P. (2009). Syntrophy in anaerobic global carbon cycles. Current Opinion in Biotechnology 20, 623-632.

Mcinerney, M.J., Sieber, J.R., and Gunsalus, R.P. (2011). Microbial syntrophy: Genomic sequences reveal systems required to produce hydrogen and formate, plus other hallmarks of the syntrophic lifestyle. Microbe 6, 479.

Mcinerney, M.J., Struchtemeyer, C.G., Sieber, J., Mouttaki, H., Stams, A.J.M., Schink, B., Rohlin, L., and Gunsalus, R.P. (2008). Physiology, ecology, phylogeny, and genomics of microorganisms capable of syntrophic metabolism. Annals of the New York Academy of Sciences 1125, 58-72.

Mcmahon, K.D., Stroot, P.G., Mackie, R.I., and Raskin, L. (2001). Anaerobic codigestion of municipal solid waste and biosolids under various mixing conditions - II: Microbial population dynamics. Water Research 35, 1817-1827.

Mcmahon, K.D., Zheng, D., Stams, A.J.M., Mackie, R.I., and Raskin, L. (2004). Microbial population dynamics during start-up and overload conditions of anaerobic digesters treating municipal solid waste and sewage sludge. Biotechnology and Bioengineering 87, 823-834.

Mcmurdie, P.J., and Holmes, S. (2013). phyloseq: an R package for reproducible interactive analysis and graphics of microbiome census data. PLOS One 8, e61217.

Mei, X., Wang, Z., Miao, Y., and Wu, Z. (2017). A pilot-scale anaerobic membrane bioreactor under short hydraulic retention time for municipal wastewater treatment: performance and microbial community identification. Journal of Water Reuse and Desalination, jwrd2017164.

Melamane, X.L., Strong, P.J., and Burgess, J.E. (2007). Treatment of wine distillery wastewater: A review with emphasis on anaerobic membrane reactors. South African Journal of Enology and Viticulture 28, 25-36.

Meyer, B., Kuehl, J.V., Deutschbauer, A.M., Arkin, A.P., and Stahl, D.A. (2013). Flexibility of syntrophic enzyme systems in Desulfovibrio species ensures their adaptation capability to environmental changes. Journal of Bacteriology 195, 4900-4914.

Milucka, J., Ferdelman, T.G., Polerecky, L., Franzke, D., Wegener, G., Schmid, M., Lieberwirth, I., Wagner, M., Widdel, F., and Kuypers, M.M. (2012). Zero-valent sulphur is a key intermediate in marine methane oxidation. Nature 491, 541-546.

Mohammadi, T., Sijbrandi, R., Lutters, M., Verheul, J., Martin, N., Den Blaauwen, T., De Kruijff, B., and Breukink, E. (2014). Specificity of the transport of Lipid II by FtsW in Escherichia coli. Journal of Biological Chemistry.

Mohana, S., Acharya, B.K., and Madamwar, D. (2009). Distillery spent wash: Treatment technologies and potential applications. Journal of Hazardous Materials 163, 12-25.

Moller, S., Croning, M.D.R., and Apweiler, R. (2001). Evaluation of methods for the prediction of membrane spanning regions. Bioinformatics 17, 646-653.

Morgan, R.M., Pihl, T.D., Nolling, J., and Reeve, J.N. (1997). Hydrogen regulation of growth, growth yields, and methane gene transcription in Methanobacterium thermoautotrophicum $\Delta \mathrm{H}$. Journal of Bacteriology 179, 889-898. 
Morikawa, M., Kagihiro, S., Haruki, M., Takano, K., Branda, S., Kolter, R., and Kanaya, S. (2006). Biofilm formation by a Bacillus subtilis strain that produces gammapolyglutamate. Microbiology 152, 2801-2807.

Moriya, H. (2015). Quantitative nature of overexpression experiments. Molecular Biology of the Cell 26, 3932-3939.

Morris, B.E., Henneberger, R., Huber, H., and Moissl-Eichinger, C. (2013). Microbial syntrophy: interaction for the common good. FEMS Microbiology Reviews 37, 384406.

Mosey, F.E., and Fernandes, X.A. (1989). Patterns of Hydrogen in Biogas from the Anaerobic-Digestion of Milk-Sugars. Water Science and Technology 21, 187-196.

Mourato, C., Martins, M., Da Silva, S.M., and Pereira, I.a.C. (2017). A continuous system for biocatalytic hydrogenation of $\mathrm{CO}_{2}$ to formate. Bioresource Technology 235, 149156.

Müller, B., Sun, L., Westerholm, M., and Schnürer, A. (2016). Bacterial community composition and fhs profiles of low- and high-ammonia biogas digesters reveal novel syntrophic acetate-oxidising bacteria. Biotechnology for Biofuels 9, 48.

Müller, N., Schleheck, D., and Schink, B. (2009). Involvement of NADH:acceptor oxidoreductase and butyryl coenzyme A dehydrogenase in reversed electron transport during syntrophic butyrate oxidation by Syntrophomonas wolfei. Journal of Bacteriology 191, 6167-6177.

Müller, N., Worm, P., Schink, B., Stams, A.J.M., and Plugge, C.M. (2010). Syntrophic butyrate and propionate oxidation processes: from genomes to reaction mechanisms. Environmental Microbiology Reports 2, 489-499.

Murto, M., Bjornsson, L., and Mattiasson, B. (2004). Impact of food industrial waste on anaerobic co-digestion of sewage sludge and pig manure. Journal of Environmental Management 70, 101-107.

Muyzer, G., and Stams, A.J.M. (2008). The ecology and biotechnology of sulphate-reducing bacteria. Nature Reviews Microbiology 6, 441-454.

Nadell, C.D., Xavier, J.B., and Foster, K.R. (2009). The sociobiology of biofilms. FEMS Microbiology Reviews 33, 206-224.

Nakanishi-Matsui, M., and Futai, M. (2006). Stochastic proton pumping ATPases: from single molecules to diverse physiological roles. IUBMB Life 58, 318-322.

Nakanishi-Matsui, M., Sekiya, M., Nakamoto, R.K., and Futai, M. (2010). The mechanism of rotating proton pumping ATPases. Biochimica et Biophysica Acta 1797, 1343-1352.

Nilsen, R.K., Torsvik, T., and Lien, T. (1996). Desulfotomaculum thermocisternum sp. nov., a sulfate reducer isolated from a hot North Sea oil reservoir. International Journal of Systematic Bacteriology 46, 397-402.

Nobu, M.K., Narihiro, T., Rinke, C., Kamagata, Y., Tringe, S.G., Woyke, T., and Liu, W.T. (2015). Microbial dark matter ecogenomics reveals complex synergistic networks in a methanogenic bioreactor. The ISME Journal 9, 1710-1722.

Offre, P., Spang, A., and Schleper, C. (2013). Archaea in biogeochemical cycles. Annual Review of Microbiology 67, 437-457.

Omil, F. (1995). Anaerobic treatment of saline wastewaters under high sulphide and ammonia content. Bioresource Technology 54, 269-278.

Ortiz-Alvarez, R., and Casamayor, E.O. (2016). High occurrence of Pacearchaeota and Woesearchaeota (Archaea superphylum DPANN) in the surface waters of oligotrophic high-altitude lakes. Environmental Microbiology Reports 8, 210-217.

Ozgun, H., Dereli, R.K., Ersahin, M.E., Kinaci, C., Spanjers, H., and Van Lier, J.B. (2013). A review of anaerobic membrane bioreactors for municipal wastewater treatment: Integration options, limitations and expectations. Separation and Purification Technology 118, 89-104.

Ozturk, S.S., Palsson, B.O., and Thiele, J.H. (1989). Control of interspecies electron transfer flow during anaerobic digestion: dynamic diffusion reaction models for hydrogen gas transfer in microbial flocs. Biotechnology and Bioengineering 33, 745-757.

Padmasiri, S.I., Zhang, J., Fitch, M., Norddahl, B., Morgenroth, E., and Raskin, L. (2007). Methanogenic population dynamics and performance of an anaerobic membrane 
bioreactor (AnMBR) treating swine manure under high shear conditions. Water Research 41, 134-144.

Paulo, L.M., Stams, A.J.M., and Sousa, D.Z. (2015). Methanogens, sulphate and heavy metals: a complex system. Reviews in Environmental Science and Bio/Technology 14, 537-553.

Paulson, J.N., Stine, O.C., Bravo, H.C., and Pop, M. (2013). Differential abundance analysis for microbial marker-gene surveys. Nature Methods 10, 1200-1202.

Pelletier, E., Kreimeyer, A., Bocs, S., Rouy, Z., Gyapay, G., Chouari, R., Riviere, D., Ganesan, A., Daegelen, P., Sghir, A., Cohen, G.N., Medigue, C., Weissenbach, J., and Le Paslier, D. (2008). "Candidatus Cloacamonas acidaminovorans": genome sequence reconstruction provides a first glimpse of a new bacterial division. Journal of Bacteriology 190, 2572-2579.

Peng, X., Zhang, Z., Luo, W., and Jia, X. (2013). Biodegradation of tetrabromobisphenol A by a novel Comamonas sp. strain, JXS-2-02, isolated from anaerobic sludge. Bioresour Technol 128, 173-179.

Pereira, I.A. (2013). An enzymatic route to $\mathrm{H}_{2}$ storage. Science 342, 1329-1330.

Pereira, I.A., Ramos, A.R., Grein, F., Marques, M.C., Da Silva, S.M., and Venceslau, S.S. (2011). A comparative genomic analysis of energy metabolism in sulfate reducing bacteria and archaea. Frontiers in Microbiology 2, 69.

Pereira, I.a.C. (2008). "Respiratory membrane complexes of Desulfovibrio," in Microbial Sulfur Metabolism, eds. C. Dahl \& C.G. Friedrich. (Berlin, Heidelberg: Springer Berlin Heidelberg), 24-35.

Peters, J.W., Miller, A.F., Jones, A.K., King, P.W., and Adams, M.W. (2016). Electron bifurcation. Current Opinion in Chemical Biology 31, 146-152.

Pires, R.H., Lourenco, A.I., Morais, F., Teixeira, M., Xavier, A.V., Saraiva, L.M., and Pereira, I.A. (2003). A novel membrane-bound respiratory complex from Desulfovibrio desulfuricans ATCC 27774. Biochimica et Biophysica Acta 1605, 67-82.

Plugge, C.M. (2005). Anoxic media design, preparation, and considerations. Methods in Enzymology 397, 3-16.

Plugge, C.M., Balk, M., and Stams, A.J.M. (2002). Desulfotomaculum thermobenzoicum subsp. thermosyntrophicum subsp. nov., a thermophilic, syntrophic, propionateoxidizing, spore-forming bacterium. International Journal of Systematic and Evolutionary Microbiology 52, 391-399.

Plugge, C.M., Dijkema, C., and Stams, A.J.M. (1993). Acetyl-CoA cleavage pathway in a syntrophic propionate oxidizing bacterium growing on fumarate in the absence of methanogens. FEMS Microbiology Letters 110, 71-76.

Plugge, C.M., Henstra, A.M., Worm, P., Swarts, D.C., Paulitsch-Fuchs, A.H., Scholten, J.C.M., Lykidis, A., Lapidus, A.L., Goltsman, E., Kim, E., Mcdonald, E., Rohlin, L., Crable, B.R., Gunsalus, R.P., Stams, A.J.M., and Mcinerney, M.J. (2012). Complete genome sequence of Syntrophobacter fumaroxidans strain (MPOB $\left.{ }^{\top}\right)$. Standards in Genomic Sciences 7, 91-106.

Plugge, C.M., Jiang, B., De Bok, F.a.M., Tsai, C., and Stams, A.J.M. (2009). Effect of tungsten and molybdenum on growth of a syntrophic coculture of Syntrophobacter fumaroxidans and Methanospirillum hungatei. Archives of Microbiology 191, 55-61.

Plugge, C.M., Scholten, J.C.M., Culley, D.E., Nie, L., Brockman, F.J., and Zhang, W. (2010). Global transcriptomics analysis of the Desulfovibrio vulgaris change from syntrophic growth with Methanosarcina barkeri to sulfidogenic metabolism. Microbiology 156, 2746-2756.

Plugge, C.M., Zhang, W., Scholten, J.C.M., and Stams, A.J.M. (2011). Metabolic flexibility of sulfate-reducing bacteria. Frontiers in Microbiology 2, 81.

Poweleit, N., Ge, P., Nguyen, H.H., Loo, R.R., Gunsalus, R.P., and Zhou, Z.H. (2016). CryoEM structure of the Methanospirillum hungatei archaellum reveals structural features distinct from the bacterial flagellum and type IV pili. Nature Microbiology 2, 16222.

Prakash, D., Wu, Y., Suh, S.J., and Duin, E.C. (2014). Elucidating the process of activation of methyl-coenzyme M reductase. Journal of Bacteriology 196, 2491-2498.

Punta, M., Coggill, P.C., Eberhardt, R.Y., Mistry, J., Tate, J., Boursnell, C., Pang, N., Forslund, K., Ceric, G., Clements, J., Heger, A., Holm, L., Sonnhammer, E.L.L., 
Eddy, S.R., Bateman, A., and Finn, R.D. (2012). The Pfam protein families database. Nucleic Acids Research 40, D290-D301.

Qiu, Y.L., Sekiguchi, Y., Hanada, S., Imachi, H., Tseng, I.C., Cheng, S.S., Ohashi, A., Harada, H., and Kamagata, Y. (2006). Pelotomaculum terephthalicum sp. nov. and Pelotomaculum isophthalicum sp. nov.: two anaerobic bacteria that degrade phthalate isomers in syntrophic association with hydrogenotrophic methanogens. Archives of Microbiology 185, 172-182.

Quast, C., Pruesse, E., Yilmaz, P., Gerken, J., Schweer, T., Yarza, P., Peplies, J., and Glockner, F.O. (2013). The SILVA ribosomal RNA gene database project: improved data processing and web-based tools. Nucleic Acids Research 41, D590-D596.

Rabus, R., Venceslau, S.S., Wohlbrand, L., Voordouw, G., Wall, J.D., and Pereira, I.A. (2015). A post-genomic view of the ecophysiology, catabolism and biotechnological relevance of sulphate-reducing prokaryotes. Advances in Microbial Physiology 66, 55-321.

Rajagopal, R., Masse, D.I., and Singh, G. (2013). A critical review on inhibition of anaerobic digestion process by excess ammonia. Bioresource Technology 143, 632-641.

Ramiro-Garcia, J., Hermes, G.D.A., Giatsis, C., Sipkema, D., Zoetendal, E.G., Schaap, P.J., and Smidt, H. (2016). NG-Tax, a highly accurate and validated pipeline for analysis of $16 \mathrm{~S}$ rRNA amplicons from complex biomes. F1000Research 5, 1791.

Ramos, A.R., Grein, F., Oliveira, G.P., Venceslau, S.S., Keller, K.L., Wall, J.D., and Pereira, I.A. (2015). The FlXABCD-HdrABC proteins correspond to a novel NADH dehydrogenase/heterodisulfide reductase widespread in anaerobic bacteria and involved in ethanol metabolism in Desulfovibrio vulgaris Hildenborough. Environmental Microbiology 17, 2288-2305.

Reda, T., Plugge, C.M., Abram, N.J., and Hirst, J. (2008). Reversible interconversion of carbon dioxide and formate by an electroactive enzyme. Proceedings of the National Academy of Sciences 105, 10654-10658.

Regueiro, L., Veiga, P., Figueroa, M., Alonso-Gutierrez, J., Stams, A.J.M., Lema, J.M., and Carballa, M. (2012). Relationship between microbial activity and microbial community structure in six full-scale anaerobic digesters. Microbiological Research $167,581-589$.

Reguera, G., Mccarthy, K.D., Mehta, T., Nicoll, J.S., Tuominen, M.T., and Lovley, D.R. (2005). Extracellular electron transfer via microbial nanowires. Nature 435, 10981101.

Rinke, C., Schwientek, P., Sczyrba, A., Ivanova, N.N., Anderson, I.J., Cheng, J.F., Darling, A., Malfatti, S., Swan, B.K., Gies, E.A., Dodsworth, J.A., Hedlund, B.P., Tsiamis, G., Sievert, S.M., Liu, W.T., Eisen, J.A., Hallam, S.J., Kyrpides, N.C., Stepanauskas, R., Rubin, E.M., Hugenholtz, P., and Woyke, T. (2013). Insights into the phylogeny and coding potential of microbial dark matter. Nature 499, 431-437.

Robinson, W.E., Bassegoda, A., Reisner, E., and Hirst, J. (2017). Oxidation-state-dependent binding properties of the active site in a Mo-containing formate dehydrogenase. Journal of the American Chemical Society 139, 9927-9936.

Romsaiyud, A., Songkasiri, W., Nopharatana, A., and Chaiprasert, P. (2009). Combination effect of $\mathrm{pH}$ and acetate on enzymatic cellulose hydrolysis. Journal of Environmental Sciences 21, 965-970.

Rotaru, A.-E., Shrestha, P.M., Liu, F., Shrestha, M., Shrestha, D., Embree, M., Zengler, K., Wardman, C., Nevin, K.P., and Lovley, D.R. (2014). A new model for electron flow during anaerobic digestion: direct interspecies electron transfer to Methanosaeta for the reduction of carbon dioxide to methane. Energy \& Environmental Science 7, 408-415.

Rotaru, A.-E., Stryhanyuk, H., Calabrese, F., Musat, F., Shrestha, P.M., Weber, H.S., Snoeyenbos-West, O.L.O., Hall, P.O.J., Richnow, H.H., Musat, N., and Thamdrup, B. (2017). Conductive particles enable syntrophic acetate oxidation between Geobacter and Methanosarcina from coastal sediments. bioRxiv.

Rotaru, A.E., Shrestha, P.M., Liu, F., Ueki, T., Nevin, K., Summers, Z.M., and Lovley, D.R. (2012). Interspecies electron transfer via hydrogen and formate rather than direct 
electrical connections in cocultures of Pelobacter carbinolicus and Geobacter sulfurreducens. Applied Environmental Microbiology 78, 7645-7651.

Ruff, A., Szczesny, J., Zacarias, S., Pereira, I.a.C., Plumeré, N., and Schuhmann, W. (2017). Protection and reactivation of the [NiFeSe] hydrogenase from Desulfovibrio vulgaris Hildenborough under oxidative conditions. ACS Energy Letters 2, 964-968.

Rupakula, A., Kruse, T., Boeren, S., Holliger, C., Smidt, H., and Maillard, J. (2013). The restricted metabolism of the obligate organohalide respiring bacterium Dehalobacter restrictus: lessons from tiered functional genomics. Philosophical Transactions of the Royal Society of London B 368, 20120325.

Sadaie, T., Sadaie, A., Takada, M., Hamano, K., Ohnishi, J., Ohta, N., Matsumoto, K., and Sadaie, Y. (2007). Reducing sludge production and the domination of Comamonadaceae by reducing the oxygen supply in the wastewater treatment procedure of a food-processing factory. Bioscience, Biotechnology, and Biochemistry 71, 791-799.

Saito, Y., Aoki, M., Hatamoto, M., Yamaguchi, T., Takai, K., and Imachi, H. (2015). Presence of a Novel Methanogenic Archaeal Lineage in Anaerobic Digesters Inferred from $<$ I $>$ mcrA $</$ I $>$ and 16S rRNA Gene Phylogenetic Analyses. Journal of Water and Environment Technology 13, 279-289.

Sakai, K., Kitazumi, Y., Shirai, O., Takagi, K., and Kano, K. (2017). Direct electron transfertype four-way bioelectrocatalysis of $\mathrm{CO}_{2} /$ formate and $\mathrm{NAD}^{+} / \mathrm{NADH}$ redox couples by tungsten-containing formate dehydrogenase adsorbed on gold nanoparticleembedded mesoporous carbon electrodes modified with 4-mercaptopyridine. Electrochemistry Communications 84, 75-79.

Santos, A.A., Venceslau, S.S., Grein, F., Leavitt, W.D., Dahl, C., Johnston, D.T., and Pereira, I.A. (2015). A protein trisulfide couples dissimilatory sulfate reduction to energy conservation. Science 350, 1541-1545.

Schink, B. (1997). Energetics of syntrophic cooperation in methanogenic degradation. Microbiology and Molecular Biology Reviews 61, 262-280.

Schink, B. (2015). Electron confurcation in anaerobic lactate oxidation. Environmental Microbiology 17, 543.

Schink, B., Montag, D., Keller, A., and Müller, N. (2017). Hydrogen or formate: Alternative key players in methanogenic degradation. Environmental Microbiology Reports.

Schink, B., and Stams, A.J.M. (2013). "Syntrophism among prokaryotes," ed. S.B. Heidelberg.), 471-493.

Schlindwein, C., Giordano, G., Santini, C.L., and Mandrand, M.A. (1990). Identification and expression of the Escherichia coli fdhD and fdhE genes, which are involved in the formation of respiratory formate dehydrogenase. Journal of Bacteriology 172, 6112-6121.

Schmidt, A., Müller, N., Schink, B., and Schleheck, D. (2013). A proteomic view at the biochemistry of syntrophic butyrate oxidation in Syntrophomonas wolfei. PLoS One 8, e56905.

Schmidt, J.E., and Ahring, B.K. (1995). Interspecies electron transfer during propionate and butyrate degradation in mesophilic, granular sludge. Applied and Environmental Microbiology 61, 2765-2767.

Schnürer, A., Zellner, G., and Svensson, B.H. (1999). Mesophilic syntrophic acetate oxidation during methane formation in biogas reactors. FEMS Microbiology Ecology $29,249-261$.

Scholten, J.C.M., and Conrad, R. (2000). Energetics of syntrophic propionate oxidation in defined batch and chemostat cocultures. Applied and Environmental Microbiology $66,2934-2942$.

Schut, G.J., and Adams, M.W. (2009). The iron-hydrogenase of Thermotoga maritima utilizes ferredoxin and NADH synergistically: a new perspective on anaerobic hydrogen production. Journal of Bacteriology 191, 4451-4457.

Seitz, H.-J., Schink, B., and Conrad, R. (1988). Thermodynamics of hydrogen metabolism in methanogenic cocultures degrading ethanol or lactate. FEMS Microbiology Letters $55,119-124$. 
Sekiguchi, Y., Kamagata, Y., Nakamura, K., Ohashi, A., and Harada, H. (2000). Syntrophothermus lipocalidus gen. nov., sp. nov., a novel thermophilic, syntrophic, fatty-acid-oxidizing anaerobe which utilizes isobutyrate. International journal of systematic and evolutionary microbiology 50 Pt 2, 771-779.

Serrano, A., Perez-Castineira, J.R., Baltscheffsky, M., and Baltscheffsky, H. (2007). $\mathrm{H}^{+}-$ PPases: yesterday, today and tomorrow. IUBMB Life 59, 76-83.

Setzke, E., Hedderich, R., Heiden, S., and Thauer, R.K. (1994). H2: heterodisulfide oxidoreductase complex from Methanobacterium thermoautotrophicum. Composition and properties. European Journal of Biochemistry 220, 139-148.

Shao, M.F., Zhang, T., and Fang, H.H. (2010). Sulfur-driven autotrophic denitrification: diversity, biochemistry, and engineering applications. Applied microbiology and biotechnology 88, 1027-1042.

Sheik, C.S., Sieber, J.R., Badalamenti, J.P., Carden, K., and Olson, A. (2017). Complete genome sequence of Desulfovibrio desulfuricans strain G11, a model sulfatereducing, hydrogenotrophic, and syntrophic partner organism. Genome Announcements 5.

Shimoyama, T., Kato, S., Ishii, S., and Watanabe, K. (2009). Flagellum mediates symbiosis. Science 323, 1574.

Shrestha, P.M., and Rotaru, A.E. (2014). Plugging in or going wireless: strategies for interspecies electron transfer. Frontiers in Microbiology 5, 237.

Shrestha, P.M., Rotaru, A.E., Aklujkar, M., Liu, F., Shrestha, M., Summers, Z.M., Malvankar, N., Flores, D.C., and Lovley, D.R. (2013). Syntrophic growth with direct interspecies electron transfer as the primary mechanism for energy exchange. Environmental Microbiology Reports 5, 904-910.

Sieber, J.R., Crable, B.R., Sheik, C.S., Hurst, G.B., Rohlin, L., Gunsalus, R.P., and Mcinerney, M.J. (2015). Proteomic analysis reveals metabolic and regulatory systems involved in the syntrophic and axenic lifestyle of Syntrophomonas wolfei. Frontiers in Microbiology 6, 115.

Sieber, J.R., Le, H.M., and Mcinerney, M.J. (2014). The importance of hydrogen and formate transfer for syntrophic fatty, aromatic and alicyclic metabolism. Environmental Microbiology 16, 177-188.

Sieber, J.R., Mcinerney, M.J., and Gunsalus, R.P. (2012). Genomic insights into syntrophy: the paradigm for anaerobic metabolic cooperation. Annual Review of Microbiology $66,429-452$.

Sieber, J.R., Sims, D.R., Han, C., Kim, E., Lykidis, A., Lapidus, A.L., Mcdonnald, E., Rohlin, L., Culley, D.E., Gunsalus, R., and Mcinerney, M.J. (2010). The genome of Syntrophomonas wolfei: new insights into syntrophic metabolism and biohydrogen production. Environmental Microbiology 12, 2289-2301.

Siegert, I., and Banks, C. (2005). The effect of volatile fatty acid additions on the anaerobic digestion of cellulose and glucose in batch reactors. Process Biochemistry 40, 34123418.

Skouteris, G., Hermosilla, D., López, P., Negro, C., and Blanco, Á. (2012). Anaerobic membrane bioreactors for wastewater treatment: A review. Chemical Engineering Journal 198-199, 138-148.

Soga, N., Kimura, K., Kinosita, K., Jr., Yoshida, M., and Suzuki, T. (2017). Perfect chemomechanical coupling of FoF1-ATP synthase. Proceedings of the National Academy of Sciences 114, 4960-4965.

Søndergaard, D., Pedersen, C.N., and Greening, C. (2016). HydDB: A web tool for hydrogenase classification and analysis. Scientific Reports 6, 34212.

Sousa, D.Z., Smidt, H., Alves, M.M., and Stams, A.J.M. (2007). Syntrophomonas zehnderi sp. nov., an anaerobe that degrades long-chain fatty acids in co-culture with Methanobacterium formicicum. International Journal of Systematic and Evolutionary Microbiology 57, 609-615.

Sousa, D.Z., Smidt, H., Alves, M.M., and Stams, A.J.M. (2009). Ecophysiology of syntrophic communities that degrade saturated and unsaturated long-chain fatty acids. Fems Microbiology Ecology 68, 257-272. 
Stams, A.J.M. (1994). Metabolic interactions between anaerobic bacteria in methanogenic environments. Antonie van Leeuwenhoek 66, 271-294.

Stams, A.J.M., De Bok, F.a.M., Plugge, C.M., Van Eekert, M.H., Dolfing, J., and Schraa, G. (2006). Exocellular electron transfer in anaerobic microbial communities. Environmental Microbiology 8, 371-382.

Stams, A.J.M., and Dong, X. (1995). Role of formate and hydrogen in the degradation of propionate and butyrate by defined suspended cocultures of acetogenic and methanogenic bacteria. Antonie van Leeuwenhoek 68, 281-284.

Stams, A.J.M., and Plugge, C.M. (2009). Electron transfer in syntrophic communities of anaerobic bacteria and archaea. Nature Reviews Microbiology 7, 568-577.

Stams, A.J.M., Plugge, C.M., De Bok, F.a.M., Van Houten, B.H.G.W., Lens, P.N.L., Dijkman, $\mathrm{H}$. , and Weijma, J. (2005). Metabolic interactions in methanogenic and sulfatereducing bioreactors. Water Science and Technology 52, 13-20.

Stams, A.J.M., Sousa, D.Z., Kleerebezem, R., and Plugge, C.M. (2012). Role of syntrophic microbial communities in high-rate methanogenic bioreactors. Water Science and Technology 66, 352-362.

Stams, A.J.M., Van Dijk, J.B., Dijkema, C., and Plugge, C.M. (1993). Growth of syntrophic propionate-oxidizing bacteria with fumarate in the absence of methanogenic bacteria. Applied and Environmental Microbiology 59, 1114-1119.

Steenbakkers, P.J.M., Geerts, W.J., Ayman-Oz, N.A., and Keltjens, J.T. (2006). Identification of pseudomurein cell wall binding domains. Molecular Microbiology 62, 1618-1630.

Stojanowic, A., Mander, G.J., Duin, E.C., and Hedderich, R. (2003). Physiological role of the $\mathrm{F}_{420}$-non-reducing hydrogenase (Mvh) from Methanothermobacter marburgensis. Archives of Microbiology 180, 194-203.

Stothard, P. (2000). The sequence manipulation suite: JavaScript programs for analyzing and formatting protein and DNA sequences. . Biotechniques 28, 1102-1104. .

Strittmatter, A.W., Liesegang, H., Rabus, R., Decker, I., Amann, J., Andres, S., Henne, A., Fricke, W.F., Martinez-Arias, R., Bartels, D., Goesmann, A., Krause, L., Puhler, A., Klenk, H.P., Richter, M., Schuler, M., Glockner, F.O., Meyerdierks, A., Gottschalk, G., and Amann, R. (2009). Genome sequence of Desulfobacterium autotrophicum HRM2, a marine sulfate reducer oxidizing organic carbon completely to carbon dioxide. Environmental Microbiology 11, 1038-1055.

Stroot, P.G., Mcmahon, K.D., Mackie, R.I., and Raskin, L. (2001). Anaerobic codigestion of municipal solid waste and biosolids under various mixing conditions - I. Digester performance. Water Research 35, 1804-1816.

Subhash, Y., Bang, J.J., You, T.H., and Lee, S.S. (2016). Description of Comamonas sediminis sp. nov., isolated from lagoon sediments. International Journal of Systematic and Evolutionary Microbiology 66, 2735-2739.

Suess, D.L., Kuchenreuther, J.M., De La Paz, L., Swartz, J.R., and Britt, R.D. (2016). Biosynthesis of the [FeFe] Hydrogenase $\mathrm{H}$ Cluster: A Central Role for the Radical SAM Enzyme HydG. Inorganic chemistry 55, 478-487.

Summers, Z.M., Fogarty, H.E., Leang, C., Franks, A.E., Malvankar, N.S., and Lovley, D.R. (2010). Direct exchange of electrons within aggregates of an evolved syntrophic coculture of anaerobic bacteria. Science 330, 1413-1415.

Sun, L., Toyonaga, M., Ohashi, A., Tourlousse, D.M., Matsuura, N., Meng, X.Y., Tamaki, H., Hanada, S., Cruz, R., Yamaguchi, T., and Sekiguchi, Y. (2016). Lentimicrobium saccharophilum gen. nov., sp. nov., a strictly anaerobic bacterium representing a new family in the phylum Bacteroidetes, and proposal of Lentimicrobiaceae fam. nov. International Journal of Systematic and Evolutionary Microbiology.

Team, R.C. (2016). "R: A language and environment for statistical computing", (ed.) R.F.F.S. Computing. (Vienna, Austria).

Thauer, R.K. (1998). Biochemistry of methanogenesis: a tribute to Marjory Stephenson. Microbiology 144 ( Pt 9), 2377-2406.

Thauer, R.K. (2012). The Wolfe cycle comes full circle. Proceedings of the National Academy of Sciences 109, 15084-15085.

Thauer, R.K., Jungermann, K., and Decker, K. (1977). Energy conservation in chemotrophic anaerobic bacteria. Bacteriological Reviews 41, 100-180. 
Thauer, R.K., Kaster, A.K., Goenrich, M., Schick, M., Hiromoto, T., and Shima, S. (2010). Hydrogenases from methanogenic archaea, nickel, a novel cofactor, and $\mathrm{H} 2$ storage. Annual Review of Biochemistry 79, 507-536.

Thauer, R.K., Kaster, A.K., Seedorf, H., Buckel, W., and Hedderich, R. (2008). Methanogenic archaea: ecologically relevant differences in energy conservation. Nature Reviews Microbiology 6, 579-591.

Thiele, J.H., Chartrain, M., and Zeikus, J.G. (1988). Control of interspecies electron flow during anaerobic digestion: Role of floc formation in syntrophic methanogenesis. Applied and Environmental Microbiology 54, 10-19.

Thiele, J.H., and Zeikus, J.G. (1988). Control of interspecies electron flow during anaerobic digestion: Significance of formate transfer versus hydrogen transfer during syntrophic methanogenesis in flocs. Applied and Environmental Microbiology 54, 20-29.

Tian, H., Fotidis, I.A., Mancini, E., Treu, L., Mahdy, A., Ballesteros, M., Gonzalez-Fernandez, C., and Angelidaki, I. (2017). Acclimation to extremely high ammonia levels in continuous biomethanation process and the associated microbial community dynamics. Bioresource Technology 247, 616-623.

Tokuda, M., Ohta, N., Morimura, S., and Kida, K. (1998). Methane fermentation of pot ale from a whisky distillery after enzymatic or microbial treatment. Journal of Fermentation and Bioengineering 85, 495-501.

Tremblay, P.L., Zhang, T., Dar, S.A., Leang, C., and Lovley, D.R. (2012). The Rnf complex of Clostridium ljungdahlii is a proton-translocating ferredoxin: $\mathrm{NAD}^{+}$oxidoreductase essential for autotrophic growth. MBio 4, e00406-00412.

Turkarslan, S., Raman, A.V., Thompson, A.W., Arens, C.E., Gillespie, M.A., Von Netzer, F., Hillesland, K.L., Stolyar, S., Lopez Garcia De Lomana, A., Reiss, D.J., GormanLewis, D., Zane, G.M., Ranish, J.A., Wall, J.D., Stahl, D.A., and Baliga, N.S. (2017). Mechanism for microbial population collapse in a fluctuating resource environment. Molecular Systems Biology 13, 919.

Ueki, A., Akasaka, H., Suzuki, D., and Ueki, K. (2006). Paludibacter propionicigenes gen. nov., sp. nov., a novel strictly anaerobic, Gram-negative, propionate-producing bacterium isolated from plant residue in irrigated rice-field soil in Japan. International Journal of Systematic and Evolutionary Microbiology 56, 39-44.

Van Den Bogert, B., De Vos, W.M., Zoetendal, E.G., and Kleerebezem, M. (2011). Microarray analysis and barcoded pyrosequencing provide consistent microbial profiles depending on the source of human intestinal samples. Applied and Environmental Microbiology 77, 2071-2080.

Van Den Bogert, B., Erkus, O., Boekhorst, J., De Goffau, M., Smid, E.J., Zoetendal, E.G., and Kleerebezem, M. (2013). Diversity of human small intestinal Streptococcus and Veillonella populations. FEMS Microbiology Ecology 85, 376-388.

Van Kuijk, B.L.M., Hagen, W.R., and Stams, A.J.M. (1998a). "Isolation and properties of the oxygen-sensitive fumarate reductase of the syntrophic propionate-oxidizing bacterium strain MPOB," in Biochemistry and bioenergetics of syntrophic propionate-oxidizing bacteria.), 71.

Van Kuijk, B.L.M., Schlosser, E., and Stams, A.J.M. (1998b). Investigation of the fumarate metabolism of the syntrophic propionate-oxidizing bacterium strain MPOB. Archives of Microbiology 169, 346-352.

Van Kuijk, B.L.M., and Stams, A.J.M. (1995). Sulfate reduction by a syntrophic propionateoxidizing bacterium. Antonie van Leeuwenhoek 68, 293-296.

Van Kuijk, B.L.M., and Stams, A.J.M. (1996). Purification and characterization of malate dehydrogenase from the syntrophic propionate-oxidizing bacterium strain MPOB. FEMS Microbiology Letters 144, 141-144.

Van Kuijk, B.L.M., Van Loo, N.D., Arendsen, A.F., Hagen, W.R., and Stams, A.J.M. (1996). Purification and characterization of fumarase from the syntrophic propionateoxidizing bacterium strain MPOB. Archives of Microbiology 165, 126-131.

Venceslau, S.S., Lino, R.R., and Pereira, I.A. (2010). The Qrc membrane complex, related to the alternative complex III, is a menaquinone reductase involved in sulfate respiration. Journal of Biological Chemistry 285, 22774-22783. 
Venceslau, S.S., Stockdreher, Y., Dahl, C., and Pereira, I.A. (2014). The "bacterial heterodisulfide" DsrC is a key protein in dissimilatory sulfur metabolism. Biochimica et Biophysica Acta 1837, 1148-1164.

Vidal, G., Carvalho, A., Méndez, R., and Lema, J.M. (2000). Influence of the content in fats and proteins on the anaerobic biodegradability of dairy wastewaters. Bioresource Technology 74, 231- 239.

Vignais, P.M., and Billoud, B. (2007). Occurrence, classification, and biological function of hydrogenases: an overview. Chemical Reviews 107, 4206-4272.

Vignais, P.M., Billoud, B., and Meyer, J. (2001). Classification and phylogeny of hydrogenases. FEMS Microbiology Reviews 25, 455-501.

Vincent, K.A., Parkin, A., and Armstrong, F.A. (2007). Investigating and exploiting the electrocatalytic properties of hydrogenases. Chemical Reviews 107, 4366-4413.

Visser, M., Worm, P., Muyzer, G., Pereira, I.A., Schaap, P.J., Plugge, C.M., Kuever, J., Parshina, S.N., Nazina, T.N., Ivanova, A.E., Bernier-Latmani, R., Goodwin, L.A., Kyrpides, N.C., Woyke, T., Chain, P., Davenport, K.W., Spring, S., Klenk, H.P., and Stams, A.J.M. (2013). Genome analysis of Desulfotomaculum kuznetsovii strain $17^{\top}$ reveals a physiological similarity with Pelotomaculum thermopropionicum strain $\mathrm{SI}^{\top}$. Standards in Genomic Sciences 8, 69-87.

Visweswaran, G.R.R., Dijkstra, B.W., and Kok, J. (2011). Murein and pseudomurein cell wall binding domains of bacteria and archaea - a comparative view. Applied Microbiology and Biotechnology 92, 921-928.

Von Canstein, H., Ogawa, J., Shimizu, S., and Lloyd, J.R. (2008). Secretion of flavins by Shewanella species and their role in extracellular electron transfer. Applied and Environmental Microbiology 74, 615-623.

Vorholt, J.A., Vaupel, M., and Thauer, R.K. (1997). A selenium-dependent and a seleniumindependent formylmethanofuran dehydrogenase and their transcriptional regulation in the hyperthermophilic Methanopyrus kandleri. Molecular Microbiology 23, 1033-1042.

Walker, C.B., He, Z., Yang, Z.K., Ringbauer, J.A., Jr., He, Q., Zhou, J., Voordouw, G., Wall, J.D., Arkin, A.P., Hazen, T.C., Stolyar, S., and Stahl, D.A. (2009). The electron transfer system of syntrophically grown Desulfovibrio vulgaris. Journal of Bacteriology 191, 5793-5801.

Wallrabenstein, C., Gorny, N., Springer, N., Ludwig, W., and Schink, B. (1995a). Pure culture of Syntrophus buswellii, definition of its phylogenetic status, and description of Syntrophus gentianae sp. nov. Systematic and Applied Microbiology 18, 62-66.

Wallrabenstein, C., Hauschild, E., and Schink, B. (1994). Pure culture and cytological properties of 'Syntrophobacter wolini'. FEMS Microbiology Letters 123, 249-254.

Wallrabenstein, C., Hauschild, E., and Schink, B. (1995b). Syntrophobacter pfennigii sp. nov., new syntrophically propionate-oxidizing anaerobe growing in pure culture with propionate and sulfate. Archives of Microbiology 164, 346-352.

Wan, C.Y., De Wever, H., Diels, L., Thoeye, C., Liang, J.B., and Huang, L.N. (2011). Biodiversity and population dynamics of microorganisms in a full-scale membrane bioreactor for municipal wastewater treatment. Water Research 45, 1129-1138.

Wang, H., Fotidis, I.A., and Angelidaki, I. (2015). Ammonia effect on hydrogenotrophic methanogens and syntrophic acetate-oxidizing bacteria. FEMS Microbiology Ecology 91.

Wang, H., Zhang, Y., and Angelidaki, I. (2016). Ammonia inhibition on hydrogen enriched anaerobic digestion of manure under mesophilic and thermophilic conditions. Water Research 105, 314-319.

Wang, S., Huang, H., Kahnt, J., Mueller, A.P., Kopke, M., and Thauer, R.K. (2013a). NADPspecific electron-bifurcating [FeFe]-hydrogenase in a functional complex with formate dehydrogenase in Clostridium autoethanogenum grown on $\mathrm{CO}$. Journal of Bacteriology 195, 4373-4386.

Wang, S., Huang, H., Kahnt, J., and Thauer, R.K. (2013b). Clostridium acidurici electronbifurcating formate dehydrogenase. Applied and Environmental Microbiology 79, 6176-6179. 
Wang, S., Huang, H., Moll, J., and Thauer, R.K. (2010). NADP ${ }^{+}$reduction with reduced ferredoxin and $\mathrm{NADP}^{+}$reduction with $\mathrm{NADH}$ are coupled via an electron-bifurcating enzyme complex in Clostridium kluyveri. Journal of Bacteriology 192, 5115-5123.

Wang, Y., and Qian, P.Y. (2009). Conservative fragments in bacterial 165 rRNA genes and primer design for $16 \mathrm{~S}$ ribosomal DNA amplicons in metagenomic studies. PLoS One 4.

Wang, Y.Y., Zhang, Y.L., Wang, J.B., and Meng, L. (2009). Effects of volatile fatty acid concentrations on methane yield and methanogenic bacteria. Biomass and Bioenergy 33, 848-853.

Westerholm, M., Moestedt, J., and Schnürer, A. (2016). Biogas production through syntrophic acetate oxidation and deliberate operating strategies for improved digester performance. Applied Energy 179, 124-135.

Wofford, N.Q., Beaty, P.S., and Mcinerney, M.J. (1986). Preparation of cell-free extracts and the enzymes involved in fatty acid metabolism in Syntrophomonas wolfei. Journal of Bacteriology 167, 179-185.

Worm, P. (2010). Formate dehydrogenases and hydrogenases in syntrophic propionateoxidizing communities: gene analysis and transcriptional profiling. PhD PhD Thesis.

Worm, P., Fermoso, F.G., Stams, A.J.M., Lens, P.N.L., and Plugge, C.M. (2011a). Transcription of fdh and hyd in Syntrophobacter spp. and Methanospirillum spp. as a diagnostic tool for monitoring anaerobic sludge deprived of molybdenum, tungsten and selenium. Environmental Microbiology 13, 1228-1235.

Worm, P., Koehorst, J.J., Visser, M., Sedano-Nunez, V.T., Schaap, P.J., Plugge, C.M., Sousa, D.Z., and Stams, A.J.M. (2014). A genomic view on syntrophic versus nonsyntrophic lifestyle in anaerobic fatty acid degrading communities. Biochimica et Biophysica Acta 1837, 2004-2016.

Worm, P., Stams, A.J.M., Cheng, X., and Plugge, C.M. (2011b). Growth- and substratedependent transcription of formate dehydrogenase and hydrogenase coding genes in Syntrophobacter fumaroxidans and Methanospirillum hungatei. Microbiology 157, 280-289.

Wu, J.H., Wu, F.Y., Chuang, H.P., Chen, W.Y., Huang, H.J., Chen, S.H., and Liu, W.T. (2013). Community and proteomic analysis of methanogenic consortia degrading terephthalate. Applied and Environmental Microbiology 79, 105-112.

Wu, L.F., and Mandrand, M.A. (1993). Microbial hydrogenases: primary structure, classification, signatures and phylogeny. FEMS Microbiology Reviews 10, 243-269.

Xie, F., Ma, H., Quan, S., Liu, D., and Chen, G. (2016). Comamonas phosphati sp. nov., isolated from a phosphate mine. International Journal of Systematic and Evolutionary Microbiology 66, 456-461.

Yadav, R.K., Baeg, J.O., Oh, G.H., Park, N.J., Kong, K.J., Kim, J., Hwang, D.W., and Biswas, S.K. (2012). A photocatalyst-enzyme coupled artificial photosynthesis system for solar energy in production of formic acid from CO2. Journal of the American Chemical Society 134, 11455-11461.

Yang, W.B., Cicek, N., and Ilg, J. (2006). State-of-the-art of membrane bioreactors: Worldwide research and commercial applications in North America. Journal of Membrane Science 270, 201-211.

Yano, T. (2002). The energy-transducing NADH: quinone oxidoreductase, complex I. Molecular Aspects of Medicine 23, 345-368.

Yenigun, O., and Demirel, B. (2013). Ammonia inhibition in anaerobic digestion: A review. Process Biochemistry 48, 901-911.

Zhang, C., Yuan, Q., and Lu, Y. (2014). Inhibitory effects of ammonia on methanogen mcrA transcripts in anaerobic digester sludge. FEMS Microbiology Ecology 87, 368-377.

Zhang, P., Yuly, J.L., Lubner, C.E., Mulder, D.W., King, P.W., Peters, J.W., and Beratan, D.N. (2017). Electron bifurcation: Thermodynamics and kinetics of two-electron brokering in biological redox chemistry. Accounts of Chemical Research 50, 24102417.

Zhang, Y., and Gladyshev, V.N. (2005). An algorithm for identification of bacterial selenocysteine insertion sequence elements and selenoprotein genes. Bioinformatics 21, 2580-2589. 
Zheng, K., Ngo, P.D., Owens, V.L., Yang, X.P., and Mansoorabadi, S.O. (2016). The biosynthetic pathway of coenzyme F430 in methanogenic and methanotrophic archaea. Science 354, 339-342.

Ziganshin, A.M., Schmidt, T., Scholwin, F., Il'inskaya, O.N., Harms, H., and Kleinsteuber, S. (2011). Bacteria and archaea involved in anaerobic digestion of distillers grains with solubles. Applied Microbiology and Biotechnology 89, 2039-2052.

Zinder, S.H. (1993). "Physiological ecology of methanogens," in Methanogenesis. Springer), 128-206.

Zinder, S.H., and Koch, M. (1984). Non-aceticlastic methanogenesis from acetate: acetate oxidation by a thermophilic syntrophic coculture. Archives of Microbiology 138, 263-272.

Zuker, M. (2003). Mfold web server for nucleic acid folding and hybridization prediction. Nucleic Acids Research 31, 3406-3415. 


\section{Summary}

Syntrophic methanogenic associations between acetogenic bacteria and methanogenic archaea are essential for the complete mineralization of organic compounds to methane and $\mathrm{CO}_{2}$. Propionate and butyrate are important intermediates in anaerobic digestion. In the absence of inorganic electron acceptors these short chain fatty acids can only be degraded if the products acetate, hydrogen and formate, are kept low by methanogens. However, when sulfate is available the conditions change, and propionate and butyrate can be oxidized coupled to sulfate reduction. Several sulfate-reducing bacteria are able to grow in syntrophic associations with methanogens, but others not.

In this thesis, a functional analysis of protein domains was performed on a selected group of bacteria with the ability to grow on short chain fatty acids alone, or in syntrophy with methanogens. Genome analysis revealed that the presence of periplasmic formate dehydrogenases, most probably involved in interspecies electron transfer, differentiated syntrophic from non-syntrophic butyrate and propionate degraders.

Moreover, the metabolic flexibility of the propionate-degrading bacterium Syntrophobacter fumaroxidans was investigated. S. fumaroxidans can couple propionate oxidation to sulfate reduction or can degrade propionate in syntrophic lifestyle with $\mathrm{H}_{2}$ and formate scavenging microorganisms. Propionate-grown cultures of S. fumaroxidans with sulfate as electron acceptor, or in syntrophy with Methanospirillum hungatei or Desulfovibrio desulfuricans were studied. We found that $S$. fumaroxidans is prone to oxidize propionate in syntrophy despite the availability of sulfate to grow on its own.

A comparative proteomic analysis of propionate degradation by $S$. fumaroxidans in five growth conditions, including axenic and cocultures, was performed. This analysis gave a thorough overview of the propionate metabolism of S. fumaroxidans. Details on the energy conservation mechanisms and electron transfer to syntrophic partners were obtained. The results indicate that confurcating hydrogenases and formate dehydrogenases are important energy converting enzymes in propionate degradation by S. fumaroxidans. Moreover, three formate dehydrogenases fulfil an important role in the syntrophic lifestyle. Furthermore, the proteomic profile of S. fumaroxidans grown with sulfate revealed in detail the sulfate respiratory pathway of this model bacterium. The abundance of a putatively confurcating protein complex detected only in sulfate-grown cells, is an important finding. This confurcating complex has similarities to heterodisulfide reductases, proteins known to bifurcate electrons in methanogenic archaea. The detection of membrane-associated proteins usually involved in sulfate reduction in all growth conditions leaves room for research on the role of these complexes in electron transfer during syntrophic lifestyle. 
Understanding the interactions between propionate-oxidizing syntrophic consortia also involved the investigation of the syntrophic partners of S. fumaroxidans. We analysed the proteome of $M$. hungatei, Methanobacterium formicicum and $D$. desulfuricans grown in syntrophy and in pure culture with $\mathrm{H}_{2} / \mathrm{CO}_{2}$ or formate. Although both methanogens can grow on hydrogen and formate, the molecular mechanisms studied in this thesis, points to the use of hydrogen in $M$. formicicum, and of formate in M. hungatei, as electron carriers in their metabolism.

Lastly, the microbial community involved in pot ale digestion in an anaerobic membrane bioreactor was analysed using 16S rRNA next-generation sequencing. The robustness of the reactor to high loading tests and the effect on the microbial composition was discussed. Moreover, on-line monitoring of hydrogen in the biogas showed a rapid response to disturbances in the proper performance of the reactor. Thus, our study supports the use of on-line $\mathrm{H}_{2}$ measurements as an early warning indicator of process instability.

The detailed study and analysis of the molecular mechanisms for energy conservation and interspecies electron transfer discussed in this thesis increases our understanding of electron fluxes occurring in methanogenic syntrophic consortia. These types of analyses are necessary to unravel the black-box ecology of anaerobic biotechnology and the global carbon flux. 


\section{Acknowledgements}

Formally I want to thank my promotor and co-promotor for working with me along this project. Caroline, thank you for giving me the opportunity to join this project and for the supervision and time you dedicated to it. I learned a lot from you since that course of Microbial Physiology that I took while doing my Master's, and later much more during all our meetings and discussions. I remember well when you interviewed me for the position, and you stated that in my CV there were only individual sports and not ones that are played in a team. I never thought about that before, and I am sure that was one of the things that I struggled with during my $\mathrm{PhD}$. But believe me, although I am a slow learner I always kept it in mind and I will be working on it. Thanks for teaching me how to be a researcher, from the theory to the practical and going through the administrative chores.

Fons, although we had many meetings along my $\mathrm{PhD}$, I am especially grateful for the discussions and prompt feedback that you gave about my manuscripts when I had already left the Netherlands. I have always been impressed by your efficient, concrete and fast reply which made the writing process easy despite the distance. Thanks for your directness, scepticism and motivation that always came when it was needed the most.

I would also like to thank Sjef Boeren and Frank van der Zee for their contributions to this work. You both were very patient and helpful, and I hope you are happy with the resulting work.

More informally I want to acknowledge HER, who is the radioactive material in my very personal Schrödinger's-like thought experiment: To have/haven't met you resulted in the superposition of "finishing this thesis on time" or "not finishing it at all". I guess that now that the box is open we see that the Wageningen interpretation had a third superposed state.

A mis padres y mis hermanos que siempre estuvieron en mi mente y en algunas ocasiones ahí conmigo. Los quiero mucho, espero que podamos estar juntos más seguido.

NEXT PAGE: During these years many people have entered, influenced or only passed by my life. To all those people that form a part of what I am today, thank you and see you along the way! 


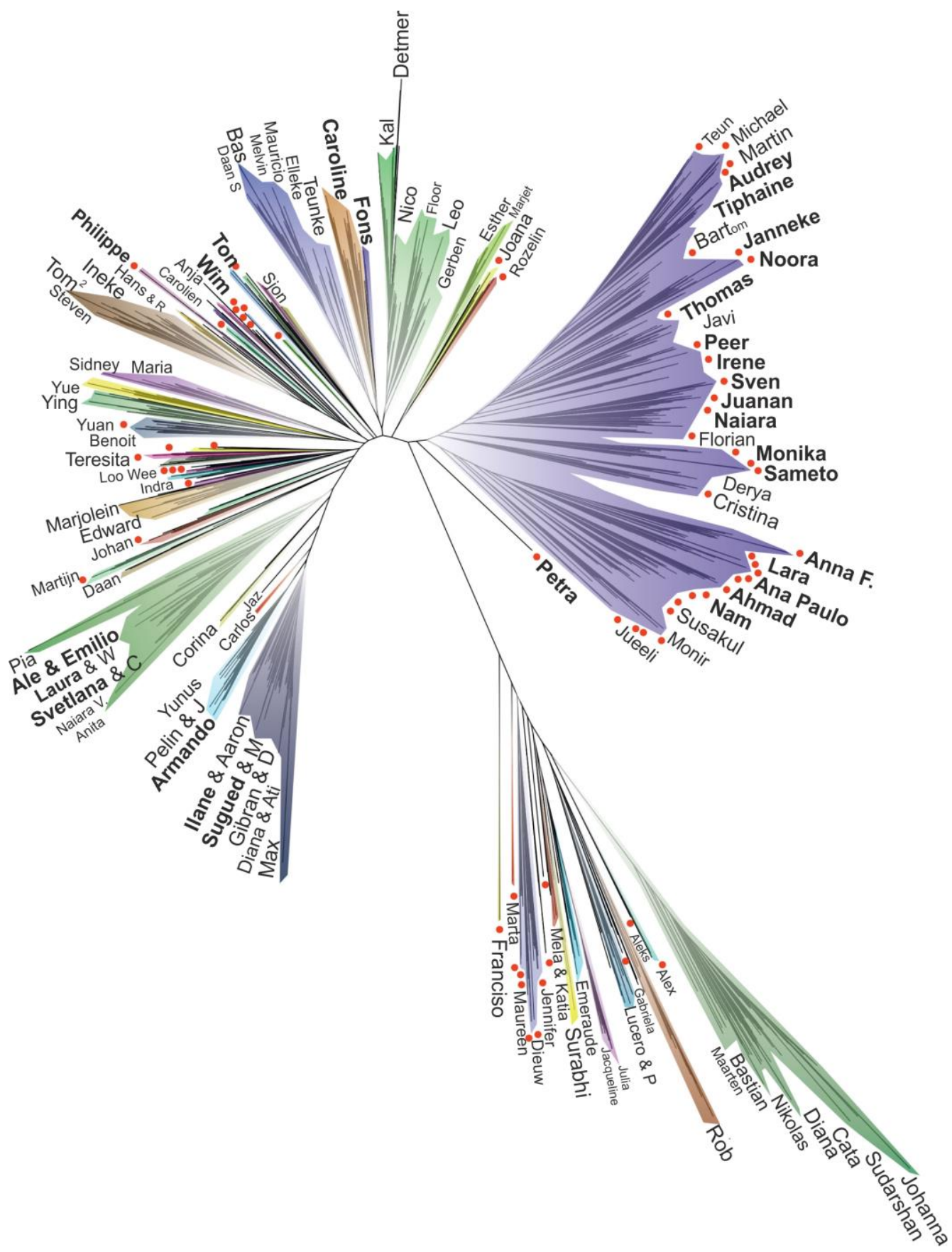

Modified from (Hug et al., 2016)

Disclaimer: No racist intention was meant in the elaboration of this diagram. For all racist interpretation you might infer from it I will argue the Tarantino effect: The racism is already there in your mind. 


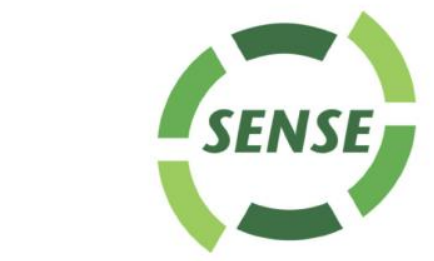

Netherlands Research School for the

Socio-Economic and Natural Sciences of the Environment

\section{I P L O M A}

For specialised PhD training

The Netherlands Research School for the Socio-Economic and Natural Sciences of the Environment

(SENSE) declares that

\section{Vicente Tonamellotl Sedano Núñez}

born on 11 August 1984 in Mexico City, Mexico

has successfully fulfilled all requirements of the Educational Programme of SENSE.

Wageningen, 3 April 2018

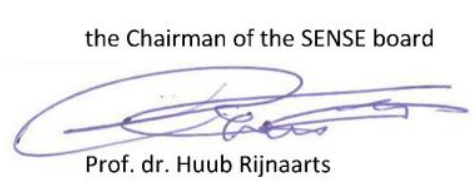

the SENSE Director of Education

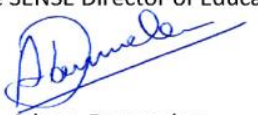

Dr. Ad van Dommelen

The SENSE Research School has been accredited by the Royal Netherlands Academy of Arts and Sciences (KNAW)

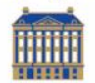

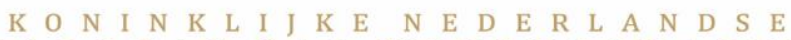

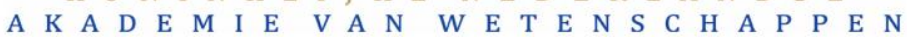




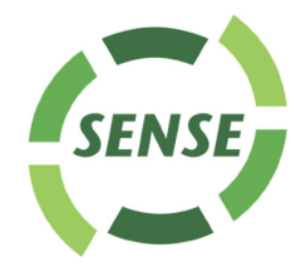

The SENSE Research School declares that Mr Vicente Tonamellotl Sedano Núñez has successfully fulfilled all requirements of the Educational PhD Programme of SENSE with a work load of $32 \mathrm{EC}$, including the following activities:

\section{SENSE PhD Courses}

- New frontiers in microbial ecology (2013)

- Environmental research in context (2012)

- Research in context activity: 'Co-organizing PhD Study Trip 2013 Laboratories of Microbiology and Systems \& Synthetic Biology (USA-Canada, 29 April - 10 May 2013)' (2013)

\section{Other PhD and Advanced MSc Courses}

- Advanced Proteomics, Wageningen University (2015)

- Scientific Publishing, Wageningen University (2011)

- Project and Time Management, Wageningen University (2011)

- Techniques for Writing and Presenting Scientific Papers, Wageningen University (2013)

\section{Management and Didactic Skills Training}

- Supervising BSc student with thesis entitled 'Reduction of bacterial growth' (2012)

- Assisting practicals of the BSc course 'Microbial Physiology' (2012-2013)

- Assisting practicals of the MSc course 'Research Methods Microbiology' (2012-2014)

\section{Oral Presentations}

- Interspecies electron transfer in fatty acid-degrading communities. Scientific Spring Meeting KNVM and NVMM, 14 April 2014, Papendal, The Netherlands

- Electron fluxes in methanogenic microbial communities, 30 April 2013, New England BioLabs, Ipswich, MA. USA.

- Metabolic flexibility of Syntrophobacter fumaroxidans, 7 May 2013, Cornell University, Ithaca, NY. USA.

- Comparative proteomic analysis of propionate oxidation by S. fumaroxidans, 10 May 2013, University of Ontario, Oshawa, ON. CANADA.

SENSE Coordinator PhD Education

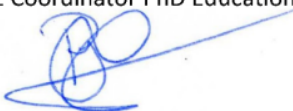

Dr. Peter Vermeulen 
This research is supported by the Dutch Technology Foundation STW, which is part of the Netherlands Organisation for Scientific Research (NWO) and partly funded by the Ministry of Economic Affairs (project number 11603)

Thesis layout: Vicente Sedano

Image in the Chapter titles: Modified section of "Man, Controller of the Universe" by Diego Rivera. 1934. Licensed under the Creative Commons Attribution-Share Alike 3.0 Unported.

Cover design: Digiforce || ProefschriftMaken

Printed by: Digiforce || ProefschriftMaken

Financial support from the Laboratory of Microbiology (Wageningen University) for printing this thesis is gratefully acknowledged. 
